\title{
Production Well Performance Enhancement using Sonication Technology Final Report
}

Period of Performance: March 25, 2002 to December 31, 2005

Michael A. Adewumi, M. Thaddeus Ityokumbul, Robert W. Watson, Eltohami Eltohami, Mario Farias, Glenn Heckman, Brendan Houlihan, Samata Prakash Karoor, Bruce G. Miller, Nazia Mohammed, Johnson Olanrewaju, Mine Ozdemir, Dautmamed Rejepov, Abdallah A. Sadegh, Kevin E. Quammie, and Jose Zaghloul, Department of Energy and GeoEnvironmental Engineering/The Energy Institute; and

\section{W. Jack Hughes and Thomas C. Montgomery, Applied Research Laboratory}

December 31, 2005

Work Performed Under Cooperative Agreement No. DE-FC26-02NT15187

For

NETL AAD Document Control, Bldg. 921

U.S. Department of Energy

National Energy Technology Laboratory

P.O. Box 10940

Pittsburgh, Pennsylvania 15236-0940

By

The Pennsylvania State University

C211 Coal Utilization Laboratory

University Park, Pennsylvania 16802 


\section{DISCLAIMER}

"This report was prepared as an account of work sponsored by an agency of the United States Government. Neither the United States Government nor any agency thereof, nor any of their employees, makes any warranty, express or implied, or assumes any legal liability or responsibility for the accuracy, completeness, or usefulness of any information, apparatus, product, or process disclosed, or represents that its use would not infringe privately owned rights. Reference herein to any specific commercial product, process, or service by trade name, trademark, manufacturer, or otherwise does not necessarily constitute or imply its endorsement, recommendation, or favoring by the United States Government or any agency thereof. The views and opinions of authors expressed herein do not necessarily state or reflect those of the United States Government or any agency thereof." 


\begin{abstract}
The objective of this project was to develop a sonic well performance enhancement technology that focused on near wellbore formation damage. In order to successfully achieve this objective, a three-year project was defined. The entire project was broken into four tasks. The overall objective of all this was to foster a better understanding of the mechanisms involved in sonic energy interactions with fluid flow in porous media and adapt such knowledge for field applications. The fours tasks are:

- Laboratory studies

- Mathematical modeling

- Sonic tool design and development

- Field demonstration
\end{abstract}

The project was designed to be completed in three years; however, due to budget cuts, support was only provided for the first year, and hence the full objective of the project could not be accomplished. This report summarizes what was accomplished with the support provided by the US Department of Energy.

Experiments performed focused on determining the inception of cavitation, studying thermal dissipation under cavitation conditions, investigating sonic energy interactions with glass beads and oil, and studying the effects of sonication on crude oil properties. Our findings show that the voltage threshold for onset of cavitation is independent of transducer-hydrophone separation distance. In addition, thermal dissipation under cavitation conditions contributed to the mobilization of deposited paraffins and waxes. Our preliminary laboratory experiments suggest that waxes are mobilized when the fluid temperature approaches $40^{\circ} \mathrm{C}$. Experiments were conducted that provided insights into the interactions between sonic wave and the fluid contained in the porous media. Most of these studies were carried out in a slim-tube apparatus.

A numerical model was developed for simulating the effect of sonication in the nearwellbore region. The numerical model developed was validated using a number of standard testbed problems. However, actual application of the model for scale-up purposes was limited due to funding constraints.

The overall plan for this task was to perform field trials with the sonication tool. These trials were to be performed in production and/or injection wells located in Pennsylvania, New 
York, and West Virginia. Four new wells were drilled in preparation for the field demonstration. Baseline production data were collected and reservoir simulator tuned to simulate these oil reservoirs. The sonication tools were designed for these wells. However, actual field testing could not be carried out because of premature termination of the project. 


\section{TABLE OF CONTENTS}

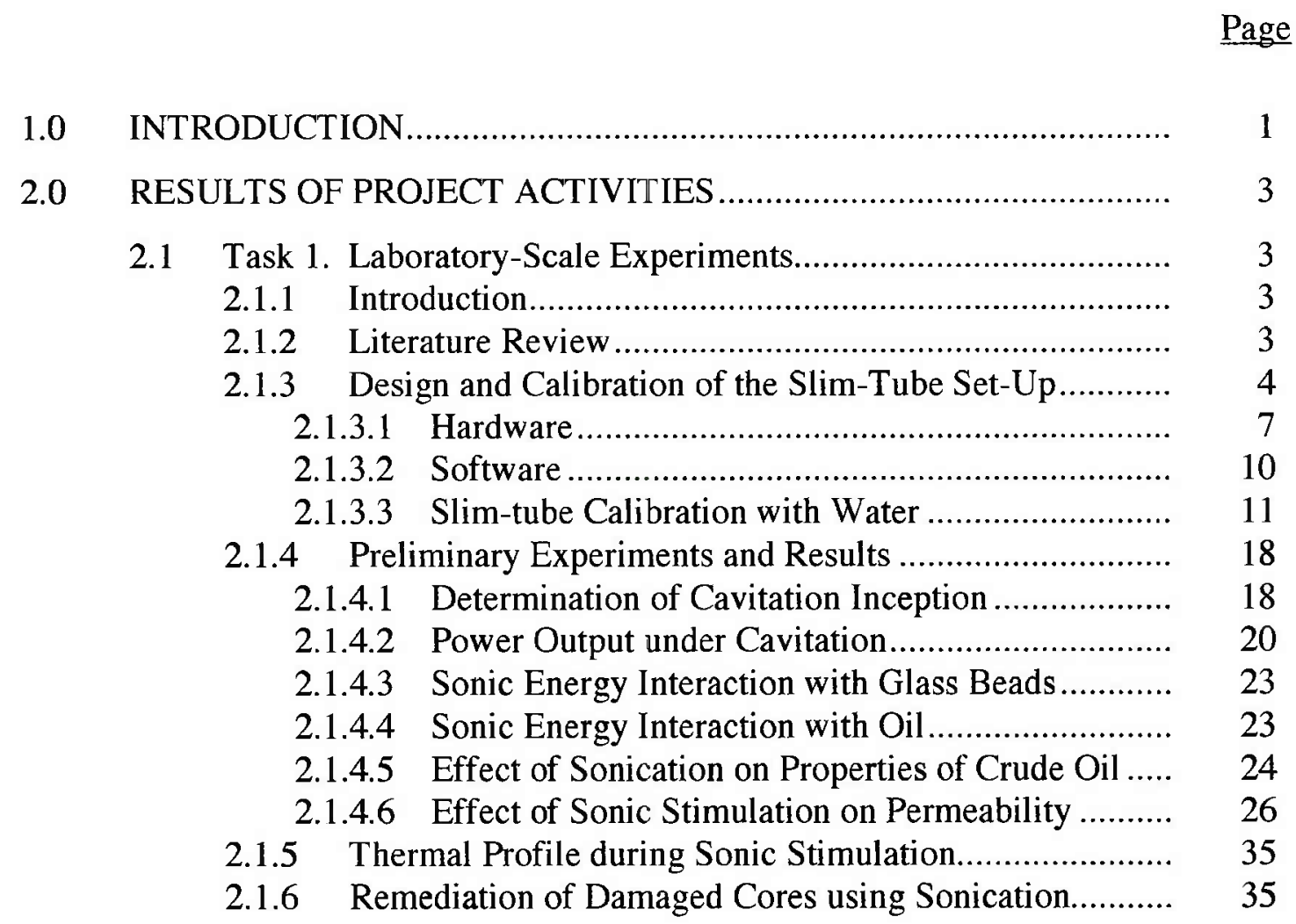

2.2 Task 2. Development of a Computer Model for Scale-Up........... 37

2.2.1 Computer Model for Scale-Up........................................ 37

2.2.1.1 Objective ................................................................ 37

2.2.1.2 Methodology ........................................................ 37

2.2.1.2.1 The General Transport Equation ...................... 38

2.2.1.2.2 Representative Elementary Volume (REV) ...... 38

2.2.1.2.3 The Averaged General Transport Equation ...... 39

2.2.1.2.4 Porous Media Formulation............................... 45

2.2.1.2.5 Solution Strategy ........................................ 57

2.2.1.2.6 Model Simplifications..................................... 58

2.2.1.3 Numerical Solver.................................................... 63

2.2.2 Numerical Model to Describe Cavitation Phenomenon... 63

2.2.3 Near-Well bore Thermal Effects of Sonication............. 65

2.2.4 Generalized Solver for Hyperbolic PDEs................ 67

2.3 Task 3. Development of a New Generation of Acoustic Transducers ................................................... 68

2.3.1 Transducers and Hydrophone Calibration ....................... 69 
2.3.2 Determination of Resonance Frequency of a Branson Horn

2.3.3 Determination of the equipment Requirement for Fabrication of the Slim-Tube Apparatus.................... 71

2.3.4 Dynamic Pressure Transducer ........................................ 71

2.3.5 Tests and Measurements............................................... 72

2.4 Task 4. Field Testing of the Sonication Technology .................... 74

2.4.1 Phase 1 Activities...................................................... 74

2.4.2 Evaluation in Partially Depleted Sandstone .................... 74

2.4.3 Evaluation in a Shot Hole ............................................... 78

2.4.4 Evaluation in a Hydro-fractured Wellbore ....................... 78

2.4.5 Evaluation in a Fractured Carbonate ................................ 78

2.4.6 Oil Wells under Water Drive .......................................... 79

2.4.7 Near-Wellbore Remediation in Stripper Wells................. 85

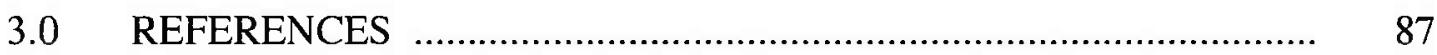

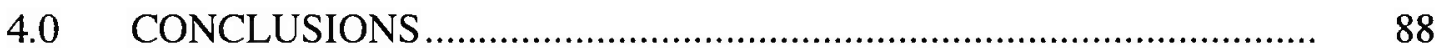

5.0 ACKNOWLEDGMENTS ............................................................. 91

APPENDIX A. LITERATURE REVIEW OF THE INFLUENCE OF ACOUTSTIC ENERGY ON PERMEABILITY OF POROUS MEDIA ……....................................................... 92

APPENDIX B. DETAILS OF MODEL DERIVATIONS ….......................... 100

APPENDIX C. LITERATURE REVIEW OF PRODUCTION FORMATIONS 137

APPENDIX D. DEVELOPMENT OF NUMERICAL SOLVER FOR WAVE PROPAGATION IN POROUS MEDIA ...................... 161

APPENDIX E. DETAILS ON THE DEVELOPMENT OF HIGH

RESOLUTION SOLVER FOR HYPERBOLIC PDES 


\subsection{Introduction}

The need for drilling new wells to increase domestic production has dominated the energy sufficiency debate for the last 30 years. This often suppresses the fact that there are millions of existing wells with a great deal of potential for enhancing production if wellbore hydraulics can be rendered more favorable. These wells are not producing at their full capacity because of flow restrictions caused by the near-wellbore formation damage. Their potential can be turned to production capacity with availability of appropriate technology. Marginal well operators, which number hundreds of thousands, cannot afford to deploy conventional technologies. Development and deployment of the right technology will improve productivity and add significantly to the nation's oil and gas resources. Flow inhibition into the wellbore may be caused by any or a combination of the following:

- solids deposition through production operations (e.g., wax, paraffin, scales, asphaltenes, hydrates, salt);

- near-wellbore effects (e.g., perforation damage, tunnel damage);

- invasion of drilling fluids and solids;

- condensate banking;

- unfavorable phase distribution (e.g., Joule-Thompson effect); and

- fines migration.

Traditionally, a number of techniques have been applied to remediate wellbore damage and enhance productivity. These include mechanical methods (e.g,. scraping), chemical injection (e.g., paraffin dispersants and acids), and thermal methods (e.g., hot oil treatment). Although these technologies are mature, they have inherent disadvantages that include high service and disposal costs, potential environmental degradation, and safety issues. Sonic technology has the potential to be an effective means of oil well productivity enhancement without negative side effects.

The objective of this project was to develop a sonic well performance enhancement technology that focused on near wellbore formations. In order to successfully achieve this objective, a three-year (i.e., three phase) project was defined with each year (phase) consisting of four tasks. The first task was a laboratory-scale study with the goal of determining the underlying principles of the technology. A scale-up mathematical model was to be developed in the second task to serve as the design guide for tool development. The third task was the development of effective transducers that can operate with variable frequency so that the most effective 
frequencies can be applied in any given situation. The system, assembled as part of the production string, was to ensure delivery of sufficient sonic energy to penetrate the near-wellbore formation. The last task was the actual field testing of the tool.

The principal goals of the project were to:

1) develop a mechanistic understanding of the enhancement impact of sonic energy on oil well productivity; and

2) develop a suitable downhole sonic device that can be deployed in the field to enhance oil well productivity through near-wellbore flow improvement.

The first phase of the project has been completed, which was performed over a two-year period, and is reported herein. The results for the four tasks are summarized in Section 2.0. References are contained in Section 3.0. Conclusions and acknowledgments are contained in Sections 4.0 and 5.0, respectively. Details of literature reviews and derivations of the modeling equations are provided in the Appendices.

The overall goals of the project were not met as the project was terminated after performing only the first of the three phases; however, the objectives of the first phase of the project were met and are reported herein. Laboratory-scale testing and modeling was performed and baseline field testing was conducted. The testing and modeling did not proceed to the point, however, were the final tool could be designed, constructed, or tested in the field since two phases of activities were not performed. 


\subsection{Results of Year 1 Activities}

\subsection{Task 1. Laboratory-Scale Experiments}

\subsubsection{Introduction}

The activities performed in Task 1 included a review of the literature to identify experimental design parameters, test variable ranges, and probable flow enhancement mechanisms. The design, fabrication and calibration of a slim-tube experimental set-up were completed. For transducers supplied by Penn State's Applied Research Laboratory (ARL) for the first phase of testing, we established the conditions for onset of cavitation - an important phenomenon in acoustic research. Using a $40 \%$ duty cycle, the specific energy dissipation rate for the $20 \mathrm{kHz}$ transducer was estimated to be approximately $82 \mathrm{~kW} / \mathrm{m}^{2}$. Preliminary experiments to elucidate acoustic energy interaction with crude oil were carried out. Chemical analysis of untreated and sonic treated Pennsylvania crude samples revealed that the aromatic components were preferentially removed during treatment. A modified combination gas/liquid permeameter (TEMCO Model GP/LP 401L) was used to study the effect of sonic stimulation on the permeability of a Berea sandstone core sample. Our results indicated that the liquid permeability was increased by over $100 \%$ with sonic stimulation and the effect appeared to be permanent in nature.

\subsubsection{Literature Review}

Empirical observations reported in the literature have indicated that immediately following a natural seismic activity, there was a significant increase in the production of crude oil and water from numerous fields in the vicinity of the seismic activity. As a result of these observations, several laboratory and field studies have been carried out in an attempt to understand how acoustic energy enhances fluid flow in porous media (see comprehensive table in Appendix A).

The effects of seismic activities were especially pronounced near inactive anticline faults. While quantitative data supporting these claims were not provided, field data have shown that under certain conditions, sonic stimulation promoted well productivity. Since the converse was also observed, research is clearly needed to delineate favorable regimes for the application of sonic stimulation in oil production. 
A review of the literature suggests that the probable mechanisms for flow enhancement with sonic stimulation may be related to:

- Removal of paraffin (waxes) and asphaltenes near the wellbore;

- Increased oil coalescence, formation pressure and/or apparent permeability;

- Reductions in oil viscosity and/or interfacial tension;

- Changes in oil-solid wettability; and

- Acoustic streaming.

Unfortunately, in many of the studies reported in Appendix A, key variables were not provided. For example, the frequency or range of frequencies used and the resulting sonic fields were not provided in approximately one-sixth of the studies. Similarly, the duration of the stimulation were not provided in almost one third of the studies. The results of these investigations show that in some instances, sonic stimulation resulted in flow enhancement while in others, the converse was true. For effective development and deployment of this technology in the field it is important that a cogent scientific explanation be provided for the observed results. It is highly probable that the inconsistencies in the empirical evidences offered as to the impact of an elastic wave on oil recovery from petroleum reservoirs may be due to differences in the experimental conditions. Currently, there are no established sets of criteria, based on scientific studies, to discriminate between reservoirs for which this method might be appropriate and the ones for which it might not apply. The goal of this study was to provide a body of scientific knowledge to evaluate and characterize sonic flooding as a viable method for improving oil recovery. The database of scientific information developed would allow one to evolve the mechanisms for this novel acoustic excitation and utilize the same as the basis for the development of design and operational models.

\subsubsection{Design and Calibration of the Slim-tube Set-up}

A slim-tube experimental system was designed to enable us to perform systematic studies on the interaction of elastic waves with fluids in porous media. The design of the set-up is similar to that used by scientists at the Los Alamos National Laboratory (Venkitaraman et al., 1994) with some modifications. For example, our design incorporates fluid chambers at both ends of the slim tube. This modification is expected to improve the coupling of the sound waves with the fluid. The length of each mixing chamber is $25.4 \mathrm{~mm}$ and its diameter will be the same as that of the slim tube $(25.4 \mathrm{~mm})$. One of these chambers houses the sonic transducer for the generation of 
sound. This design was to be used to study both concurrent and countercurrent arrangements for sonic excitation and fluid flow. The setup is designed for the inlet and effluent fluid lines to be interchanged when conducting experiments with sonic energy and fluid flow in either concurrent or countercurrent arrangements. In addition, the inlet and the outlet flow lines are fitted with pressure transducers to monitor pressure differentials at the two terminals. The clustering of the first three sensors is intended to elucidate changes in acoustic intensity in the vicinity of the transducer. Initial acoustic intensity, Io, near the tip of the transducer will be determined without the packing materials and the wave field intensity, I, spanning the length of the packing material will be measured and used to determine the effective penetration depth of the sonic excitation. The system can be operated under pressure up to 600 psig.

Figure 2.1.1 shows the block and schematic diagrams of the instrumentation for the prototype slim tube. All the sensors and connections were hermetically positioned with reference to the distance from the shaker's piston head. A shaker generates acoustic plane waves. Copperconstantan thermocouples (Omega Engineering model TMQSS-125U), dynamic pressure sensors (PCB Piezotronics model 112A22) and static pressure transducers (Omega Engineering model PX01C1-KG5T) were mounted on the slim tube at $120^{\circ}$ to each other with their tips flush with the inner wall of the slim tube. The insert of Figure 2.1.1 shows the geometric arrangement of dynamic pressure transducer, thermocouple, and static pressure sensor. The temperature, dynamic pressure, and static pressure sensors have ranges from -20 to $300^{\circ} \mathrm{C}, 0$ to 150 psi-peak and 0 to 1000 psi, respectively. A PVDF with FET hydrophone, which was fabricated and calibrated in an anechoic tank facility at ARL, was connected to the end the of the slim tube, 121 $\mathrm{cm}$ from the shaker. A Hewlett Packard (HP) power supply model 6215A was used to supply 5 DC volts to the hydrophone. Sensors were connected to the IOTEC WaveBook/516E or WaveBook (WK18) signal analyzer and signal conditioning module. Table 2.1.1 shows materials selection and property required for the construction of the slim-tube apparatus. 


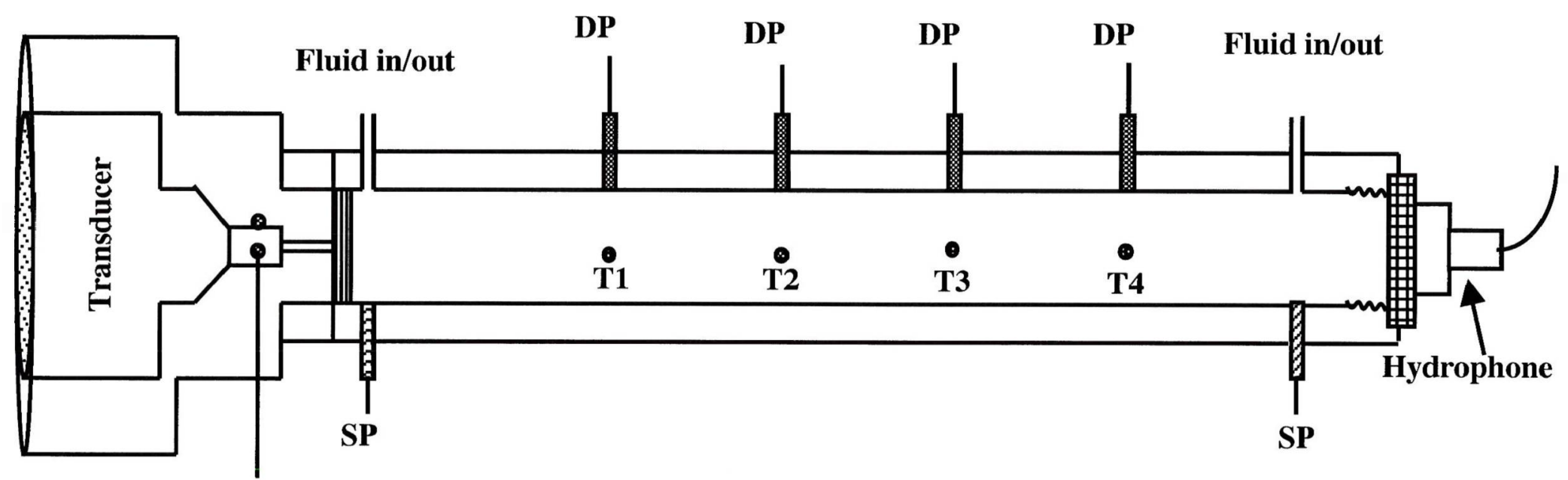

Acceleromete

DP = Dynamic pressure transducer

SP = Static pressure sensor

$\mathbf{T}=$ Thermocounle

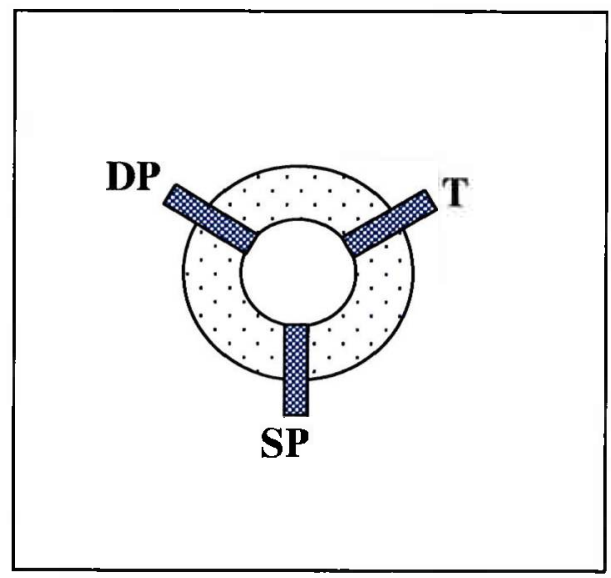

Figure 2.1.1 The block diagram of the slim-tube apparatus. The geometric arrangement of the dynamic pressure sensor, thermocouple and static pressure transducers are shown in the insert located at the bottom right corner 
Table 2.1.1 Materials property required for a slim-tube apparatus

Material

Transducer- sound wave source

Receiver (hydrophones)

Stainless-steel tube

Media

Mesh
Criterial factor for selection

Frequency $100 \mathrm{~Hz}$ to $60 \mathrm{kHz}$; operating temperature 25 to $100^{\circ} \mathrm{C}$; pressure $600 \mathrm{psi}$; piston/radiating head $<25.4 \mathrm{~mm}$.

Frequency $100 \mathrm{~Hz}$ to $60 \mathrm{kHz}$; operating temperature 25 to $100^{\circ} \mathrm{C}$; pressure 600 psi or at initial burst pressure; millimeter size.

Variable lengths $(8,24,36$, and 48 "), ID 1.0 " OD 1.5"; thickness 0.25 "; operating temperature 25 to $100^{\circ} \mathrm{C}$; $600 \mathrm{psi}$.

Rounded and surface methylated glass beads, sand (silica), alumina particle size (mesh) 50-60, 60-70, 70-80, 80-100. Tests with both hydrophilic and hydrophobic glass beads as packing material will allow us to determine the effect of rock wettability on fluid (water, brine, crude oil, Soltrol 170, crude oil or a combination) flow in the presence of acoustic energy.

Stainless-steel filter, low-acoustic impedance or thickness less than operating wavelength, pore size smaller than the smallest particle size.

\subsubsection{Hardware}

\section{WaveBook 516E and WaveBook WKB18}

Both signal analyzer and signal conditioning module are used to receive input and display output signals from the sensors. They are capable of functioning as a programmable pre- and post-filtering sampling rates and simultaneous sampling of pulse frequency. Oscilloscope and dynamic signal analyzer of the hardware functions can be used to monitor changes in amplitudes, estimate speed of sound, and preview waveforms features such as reflection or a phenomenon of beat frequency. Data extracted from the scope function can be used to set threshold values of the amplitudes of the accelerator, 
dynamic pressure transducers and hydrophone during data analyses with MATLAB software.

\section{Function Generator}

Hewlett Packard model 3314A. This equipment serves as a power generator, inputs for analog trigger of the WaveBook/516E and the oscilloscope. Parameters such as frequency, amplitude, pulse repetition time, and duty cycles can be input and adjusted as needed.

\section{Oscilloscope}

Philips model PM 3261. It is mainly used to verify input/output signals from the power generator and output signals from the sensors. The amplitudes of the signals can be controlled by separate gain knobs.

\section{Power Amplifier}

Wilcoxon Research model PA7D. The power amplifier amplifies the AC voltage input from the generator and feeds it to the matching network. It is a dual channel amplifier specifically designed to operate the F4/F7 shaker. The two channels can be operated in tandem or independently to operate the $\mathrm{F} 4, \mathrm{~F} 7$, or the combination. Both channels are driven by a common oscillator input with the amplitude controlled by separate gain knobs situated in the front panel. Channel A powers the F4 shaker, has a $15 \mathrm{~Hz}(-3 \mathrm{~dB}$ point) high pass filter and channel B powers the F7 shaker, set at $800 \mathrm{~Hz}(-3 \mathrm{~dB}$ point).

\section{Matching Network}

Wilcoxon Research model N7C. The matching network is connected between the power amplifier and the transducer. By selecting various impendence taps on the transformer, the power factor to the transducer is matched so that more power can be sent to the transducer. It is designed to power the combination shaker (F4/F7). It supplies a maximum of 42 Vrms from the F4 shaker channel output with selectable maximum voltage range of $200,300,500$ and $800 \mathrm{Vrms}$ from the F7 output. 


\section{Transducers (projector}

Three ARL-fabricated tonpilz transducers were supplied: two rated at $20 \mathrm{kHz}$ and one at $40 \mathrm{kHz}$. The tonpilz is a longitudinal vibrator designed for relatively low frequency, high power transducers that derives its name from two German words "sound" and "mushroom" or "singing mushroom". Its name reflects its configuration (Figure 2.1.2). The transducer consists of a piezoceramic ring stack between massive ends, prestressed by a central ceramic. Tail and head masses lower the device's resonance frequency well below that of the piezo stack. Prestress permits high intensity drive and output. The lighter head mass is flared for better mechanical impedance matching to the load and to size the radiating aperture for beam collimation.

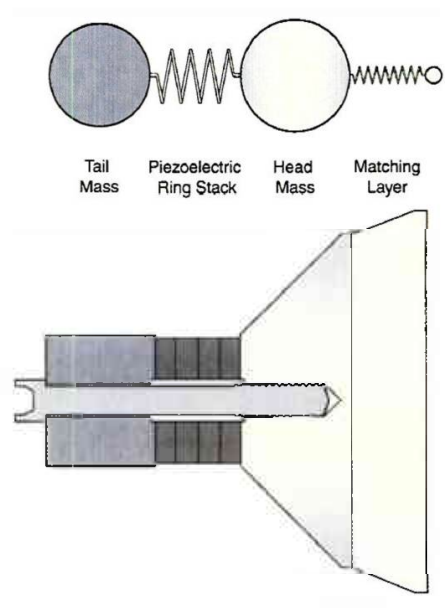

Figure 2.1.2 Tonpilz tranducer cross-section and accompanied spring-mass model

We acquired three off-the-shelve cup-horn high-intensity, flow-thru and cell distruptor transducers from Branson Sonifier. These three projectors are powered by a 250 watts power supply. They are designed to cavitate at all operating conditions; however, with a different power amplifier and hardware, we can operate them below the cavitation regime.

\section{Hydrophones}

Two polyvinyliden fluoride (PVDF) with FET hydrophones were supplied by ARL. These hydrophones were fabricated and characterized in their laboratories. One of 
the hydrophones is rectangular in shape while the other is circular in shape and is $\mathbf{2 5 . 4}$ $\mathrm{mm}$ in diameter with a thickness of $6.4 \mathrm{~mm}$. The FET hydrophone is connected to the opposite end of the slim tube to receive sound waves transmitted from the shaker.

\section{Shaker}

The Wilcoxon F4/F7 Shaker is a wide range frequency vibration shaker, driven by a rod firmly attached to a piston $(<25.4 \mathrm{~mm})$ and threaded to a tapped -inch hole with a removable stud support. The main body is $7.62 \mathrm{~cm}$ long and $13 \mathrm{~cm}$ in diameter. It consists of electromagnetic shaker (F4) for operation from low to moderately (10-7500 $\mathrm{Hz}$ ) high frequencies and piezoelectric shaker (F7) from medium to ultrasonic (500$20,000 \mathrm{~Hz}$ ) frequencies. F7 is mounted concentrically with the F4 shaker. For wide band frequency sweeps, both shakers can be driven independently or in tandem. When operated in tandem, force output of the low frequency electromagnetic generator drops off, the force output of the piezoelectric generator increases for a smooth crossover between vibrator for automatic frequency sweep applications. For independent operation, force output signal can be maximized over smaller frequency bandwiths. The signal is fed into a power amplifier and then either directly to the electromagnetic shaker or through a matching network to the piezoelectric shaker. There are one acceleration and a force sensing interfaces. A piezoelectric accelerometer $(6 \mathrm{pC} / \mathrm{g}$ nominal sensitivity) and a piezoelectric force gage with a nominal sensitivity of $200 \mathrm{pC} / \mathrm{Ib}$ Model Z7 Transducer Base is used with the F7/F4 combination. With sinusoidal force input, mechanical impedance of the structure can be calculated from the values of applied force gage output and the resulting motion from the accelerator output. The whole shaker system is housed and supported by two concentric rings and a tubular steel case. The F4/F7 can be operated in either continuous wave or in pulse mode.

\subsubsection{Software}

\section{WaveView Software}

WaveView software, which accompanied the WaveBook, was designed for setup, acquisition, and real-time display in oscilloscope mode for real-time waveform display and logger mode for continuous streaming to disk. The optional eZ-Analyst can be 
programmed for real-time spectrum analysis including drivers for Visual Basic ${ }, \mathrm{C}++$, MATLAB®, and DasyLab for Windows ${ }^{\circledR}$ XP and Windows 2000 platforms.

\section{MATLAB and Toolbox}

Toolbox algorithms enhance MATLAB's functionality in domains such as signal and image processing, data analysis and statistics, mathematical modeling, and control design. Toolboxes are collections of algorithms, written by experts in their fields, that provide application-specific numerical, analysis, and graphical capabilities. MATLAB version 6.5 algorithms can be customized and optimized toolbox functions were used for our project requirements. The WaveBook 516E WaveBook (WK18) hardware and software are controlled via 10/100BaseT Ethernet interface card.

\subsubsection{Slim-Tube Calibration with Water}

The objective of this study was to calibrate the slim tube with sound waves traveling through the tube and analyze various features of the spectra. The information obtained will be used to extend and determine usable frequencies that will result in maximum power generation and delivering in the wellbore.

\section{Methodology}

Scope trace signals were generated from 1 to $30 \mathrm{kHz}$ at pulse lengths of $1,000 \mathrm{~s}$ with the following parameters:

Frequency $=1$ to $30 \mathrm{kHz}$

Pulse repetition time $=10 \mathrm{~ms}$ Sampling frequency $=100 \mathrm{kHz}$ Pulse length $=20,800$, and 2000 s Medium = water
Tube hydrostatic pressure $=70 \mathrm{psi}$

Number of samples $=500$

Wilcoxon Amplifier setting $=300 \mathrm{Vrms}$

$\mathrm{s} \quad$ Function generator $=2 \mathrm{Vrms}$

Duty cycles (\%) were calculated with respect to the pulse length, period and number of cycles for each excitation frequency. Filter cutoff frequency was determined according to the operating frequency. For example, at $15 \mathrm{kHz}$, the filter was set at 18 $\mathrm{kHz}$. The main function of the filter is to improve signal-to-noise ratio. A test matrix for the slim-tube calibration was derived from the above parameters. Data were collected with the shaker turned off to record information about the background noise originating 
from the surrounding. Data were collected and stored in a hard drive for further processing with the MATLAB program.

\section{Results}

Oscilloscope signals with the shaker turned off (no signal) revealed an unknown background noise with an inherent frequency of about $10 \mathrm{kHz}$. The shaker's magnetorestrictive and piezoelectric transducers frequency cross-over was determined from the output amplitude as a function of frequency (Figure 2.1.3). The figure shows that cross-over occurs at about $2 \mathrm{kHz}$. This implies that the maximum power output (amplitude) is obtained from the magnetic shaker. Above $2 \mathrm{kHz}$ frequency, it is advisable to use the piezoelectric transducer.

Both static pressure (SP1 and SP2) transducers registered about $8 \times 10^{5} \mathrm{~Pa}$ pressure and approximately $66^{\circ} \mathrm{F}$ for both thermocouples (T1 and T2) throughout the test. Spectra were examined for the presence of reflections, reverberations, attenuation, and change in amplitude. The reflected spectra were of lesser amplitudes than the incident spectrum. The relative amplitude of a reflected wave is governed by the acoustic impedances of the materials involved. If absorbing media are present, the reflected waves will be of lower amplitudes and vice versa. The FET hydrophone showed the largest ratio of reflected to incident wave amplitude. Some oscilloscope waveforms also displayed beat frequencies. The phenomenon of beat occurs when temporal interference of two sound waves having slightly different frequencies display successions of constructive maximum and destructive minimum amplitude spaced in time.

Several quantitative and qualitative observations were made from the time history (amplitude as a function of period or time) of the oscilloscope function of the WaveBooks for the voltage and pressure amplitudes of the accelerometer, hydrophone and dynamic pressure sensors (Figure 2.1.4). The horizontal axis (time or period) consists of 25 divisions ( $5 \mathrm{~ms}$ ), a value of $200 \mathrm{~s}$ /division. Amplitudes of the sensors are represented on the y-axis. The figure shows time lag among sensors as a function of time and distance from the piston's head (Table 2.1.2). 


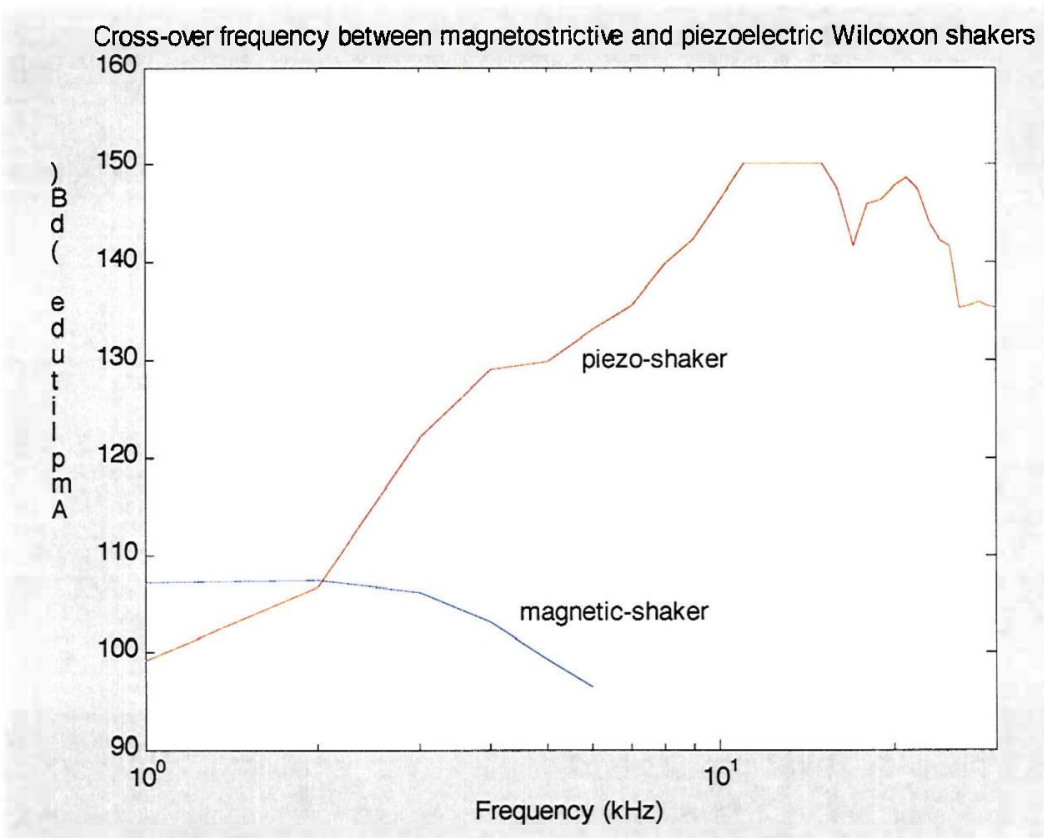

Figure 2.1.3 Piezo-magneto shaker's crossover frequency determined as a function of input power

The travel time of the acoustic waves between each dynamic sensor is approximately 140 microseconds. This constant value was used to calculate and verify the speed of sound in water $(\mathrm{Cw})$. For example, the speed of sound between DP3 and DP4 is $1,500 \mathrm{~m} / \mathrm{s}\left(\mathrm{Cw}(\mathrm{m} / \mathrm{s})=21 \times 10^{-2} \mathrm{~m} / 140 \times 10^{-6} \mathrm{~s}=1500 \mathrm{~m} / \mathrm{s}\right.$, which is calculated from Table 2.1.2). Threshold values of the amplitudes for the dynamic pressure sensors and hydrophones were recorded from the scope trace signals. These values were input for data analysis.

Radical turn on/off transient in the form of reduced amplitude was observed from some of the shaker's accelerator spectra between 400-600 $\mathrm{s}$ as shown in Figure 2.1.4. These may be caused by the reflections from water behind the piston, leading to destructive interference of the incident signals. At 30 cycles, the pulse length $(2000 \mathrm{~s})$ terminated exponentially, a sign of a long ring down caused by the shaker's high Q. A ring down phenomenon occurs when the transducer completes vibration at the end of a set pulse length or cycles, the amplitude decreases exponentially instead of an abrupt and a sharp termination of sinusoidal signals. 


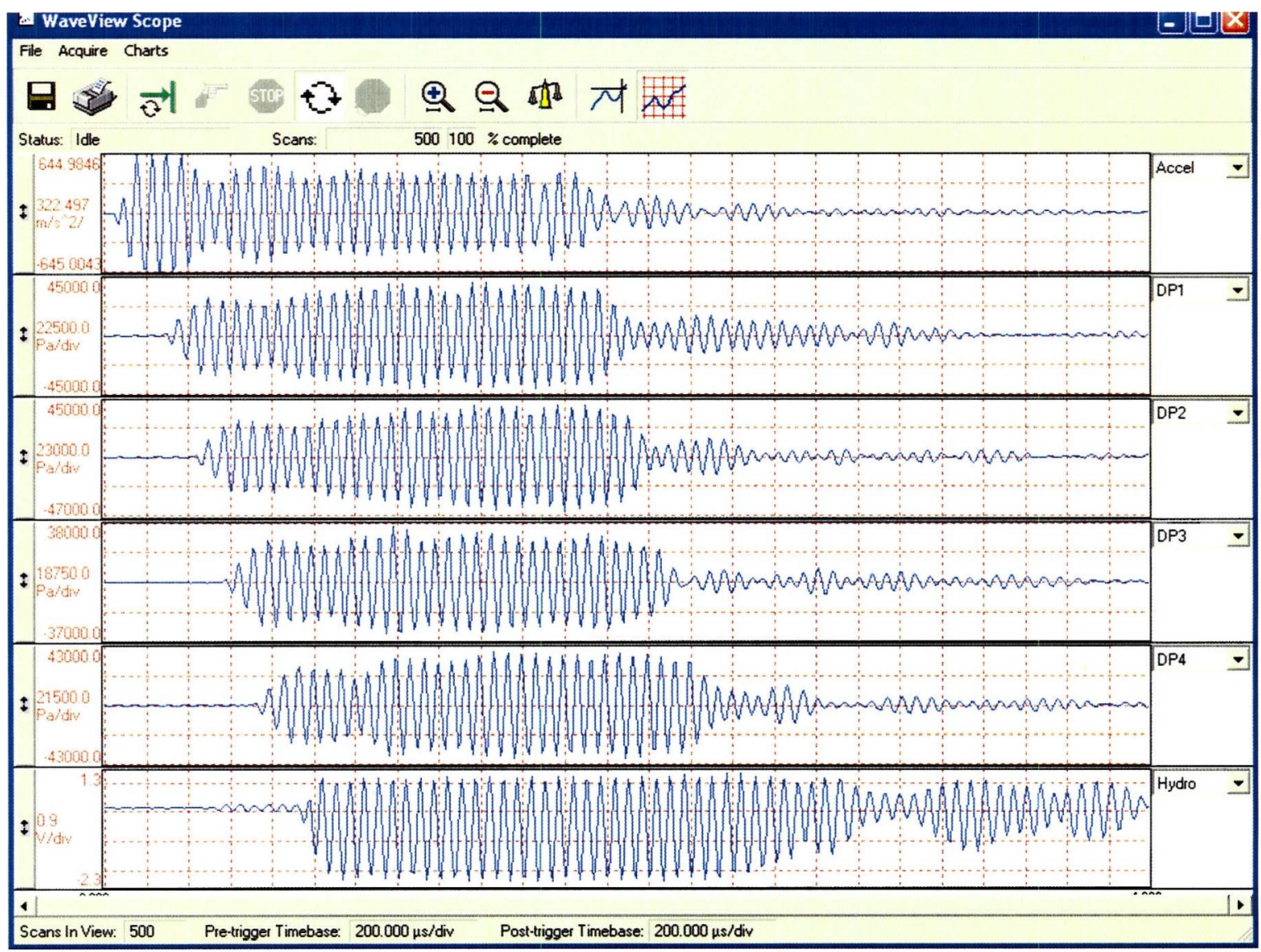

Figure 2.1.4 Oscilloscope trace obtained at frequency $=15 \mathrm{kHz}, \mathrm{N}=30$, pulse length $=$ $2000 \mathrm{~s}$ and repetition rate $=10 \mathrm{~ms}$

Table 2.1.2 Oscilloscope data obtained at $20 \mathrm{kHz}$ frequency, amplitude $=2 \mathrm{Vrms}, 10$ cycles and at room temperature and pressure

\begin{tabular}{|c|c|c|c|}
\hline Sensor & Time (s) & Distance (m) & Amplitude \\
\hline & & & \\
\hline Accelerator & $140 \times 10^{-2}$ & 0 & $0.1685 \mathrm{Vrms}$ \\
\hline DP1 & $350 \times 10^{-6}$ & $14.5 \times 10^{-2}$ & $4903.86 \mathrm{~Pa}$ \\
\hline DP2 & $490 \times 10^{-6}$ & $35.5 \times 10^{-2}$ & $4169.33 \mathrm{~Pa}$ \\
\hline DP3 & $640 \times 10^{-6}$ & $66.5 \times 10^{-2}$ & $3956.76 \mathrm{~Pa}$ \\
\hline DP4 & $780 \times 10^{-6}$ & $87.5 \times 10^{-2}$ & $4253.52 \mathrm{PA}$ \\
\hline Hydrophone & $1030 \times 10^{-2}$ & $122 \times 10^{-2}$ & $0.391 \mathrm{Vrms}$ \\
\hline
\end{tabular}

All the spectra recorded below $10 \mathrm{kHz}$ excitation frequencies suffered from elongation of signals due to reflections. This is especially more pronounced at $5 \mathrm{kHz}$ excitation frequency, which is the natural resonance frequency of the slim tube apparatus. Furthermore, this may be due to improper matching network, appearance of high 
frequency, and double harmonics. The signal due to the tube resonating is amplified in the FET hydrophone time delay signal before the incident plane waves from the shaker is sensed, whereas these signals were suppressed in the four dynamic pressure sensors' signals. Dynamic sensor transducers have low acceleration sensitivity, that is, acceleration cancellation. The spectra obtained between $2-10$ and above $20 \mathrm{kHz}$ excitation frequencies were muffled up in reflection, reverberation due to destructive interference and impedance mismatch between the shaker's piezoelectric transducer and the matching network. The shaker may also have been driven beyond its capable frequency range of $20 \mathrm{kHz}$.

Pulse lengths of reflected sound waves are mostly factors of the pulse length of the tube, $810 \mathrm{~s}$. This observation suggests that reflection originated from the shaker's piston of the end of the tube because all the sensors were flush-mounted on the tube wall. When a reflected sound wave hits a solid object, the reflected wave is $180^{\circ}$ out of phase with the incident wave and results in interference destruction. Consequently, the amplitude tends to decrease, requiring more input power to compensate for the loss.

Figure 2.1.5 shows the output of the processed data of accelerator, dynamic pressures and the FET hydrophone. Figure 2.1.5a consists of the acoustic power measured from the accelerator and dynamic pressure outputs. The maximum peak intensities correspond to the excitation frequency of $15 \mathrm{kHz}$. Figure $2.1 .5 \mathrm{~b}$ is the linear average time history of Figure 2.1.5a. The values indicate the average power or intensity measured from the accelerator and the four dynamic pressure sensors. The dynamic pressure sensor number 3 has the lowest value among the four sensors because it was defective. Constant value of the intensities indicates that the amplitude of sound wave is not diminished by reflection or other obstacle. The values of the intensities as a function of the excitation frequencies (1-30 kHz) similar to Figure 2.1.5 are summarized in Figures 2.1.6a and 2.1.6b for the input power and three dynamic pressure sensors. The maximum output of shaker's driving power is obtained between 11 and $13 \mathrm{kHz}$ and correspondingly measured by the dynamic sensors. The usable frequencies that will result in maximum power range from $11-16 \mathrm{kHz}$. 

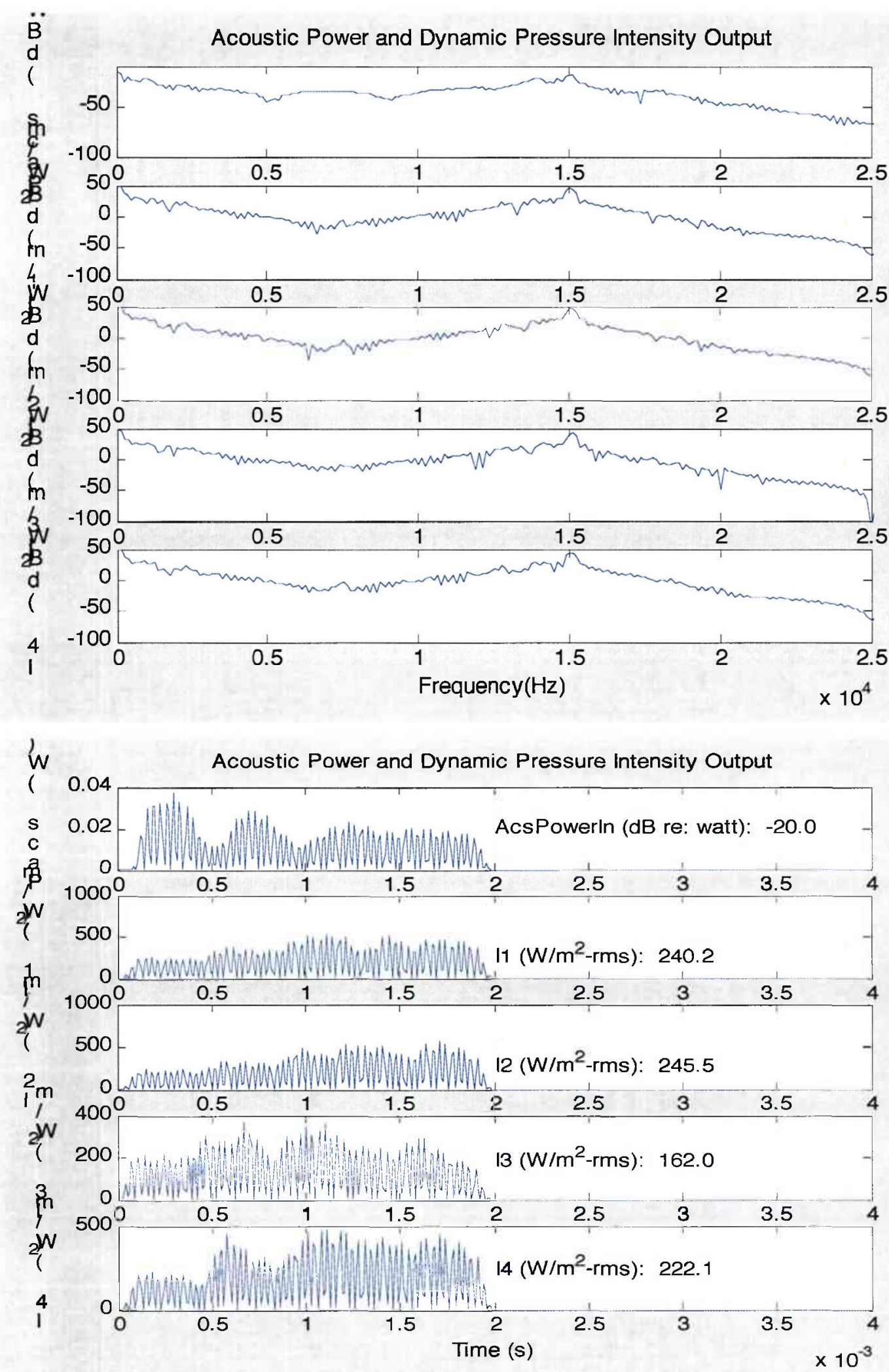

Figure 2.1.5 Output of the acoustic input power obtained from the accelerator and pressure intensity output for the four dynamic pressure sensors as functions of frequency (a) and time (b) 

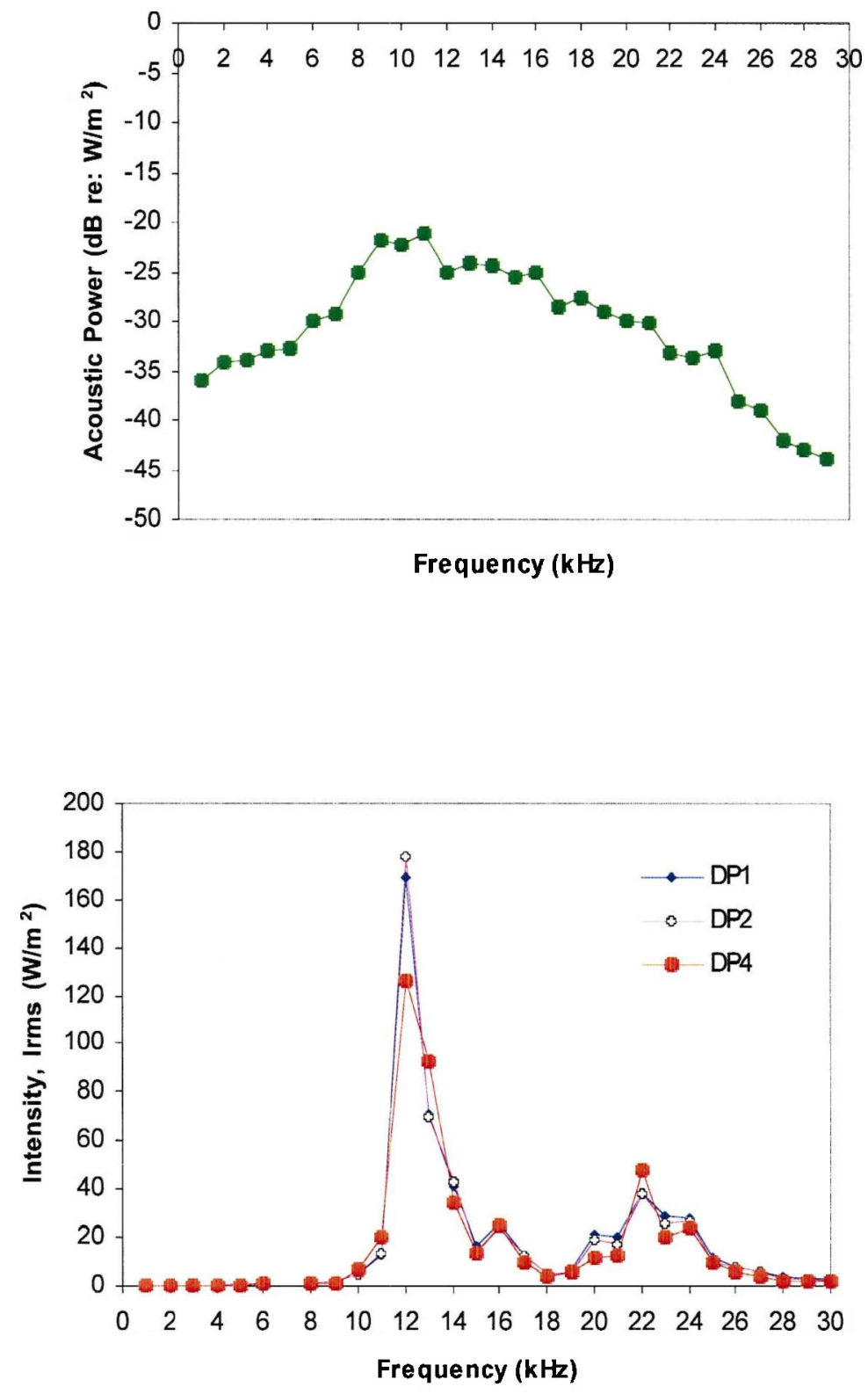

Figures 2.1.6a and 2.1.6b Input acoustic power and dynamic pressure intensities as a function of excitation frequency 


\subsubsection{Preliminary Experiments and Results}

\subsubsection{Determination of Cavitation Inception}

Our initial focus was on determining the operational characteristics of the two transducers provided by ARL. Our goal was to delineate the conditions for onset of cavitation. Our second goal was to determine the power dissipation rate during cavitation. In the special case where the near wellbore damage is due to deposition of waxes and/or asphaltenes, we believe that sonic stimulation will be enhanced when the device is operated under cavitation conditions. During cavitation, the energy dissipated is focused in a narrow zone and the heat generated may promote the mobility of the waxes and/or asphaltenes.

\section{Procedure}

The experimental setup used to determine the onset of cavitation is shown in Figure 2.1.7. A lead urethane (PU) block was suspended in a $3.5 \mathrm{~L}$ beaker filled with tap water from three chains attached to a hook. The hook was connected to a gear mechanism, which was used to alter the transducer-hydrophone separation distance. A hydrophone whose sensitivity was previously determined at ARL was attached to the top of the PU block and was connected to the HP function generator (Analyzer). During operation, the analyzer displays the operating frequency, hydrophone output drive level voltage ( $\mathrm{dB}$ Vrms) and total harmonic distortion.

At a fixed frequency and transducer input voltage, the corresponding current was read from the oscilloscope. Sound pressure exerted on the hydrophone by the transducer was obtained from the HP Analyzer as receive drive level voltage. From the input drive voltage, the transducer's drive level voltage was calculated. The experimental conditions employed are:

Matching network setting $=500 \mathrm{Vrms}$

Pulse length $=0.004 \mathrm{sec}$ or Duty cycle $0.40 \%$

Frequency: 20, 15, 10 and $5 \mathrm{kHz}$

Transducer head-hydrophone distance $=8,12$, or $16 \mathrm{~cm}$
Power Amplifier $=$ variable

Amplitude $=3.50 \mathrm{~V}$ 


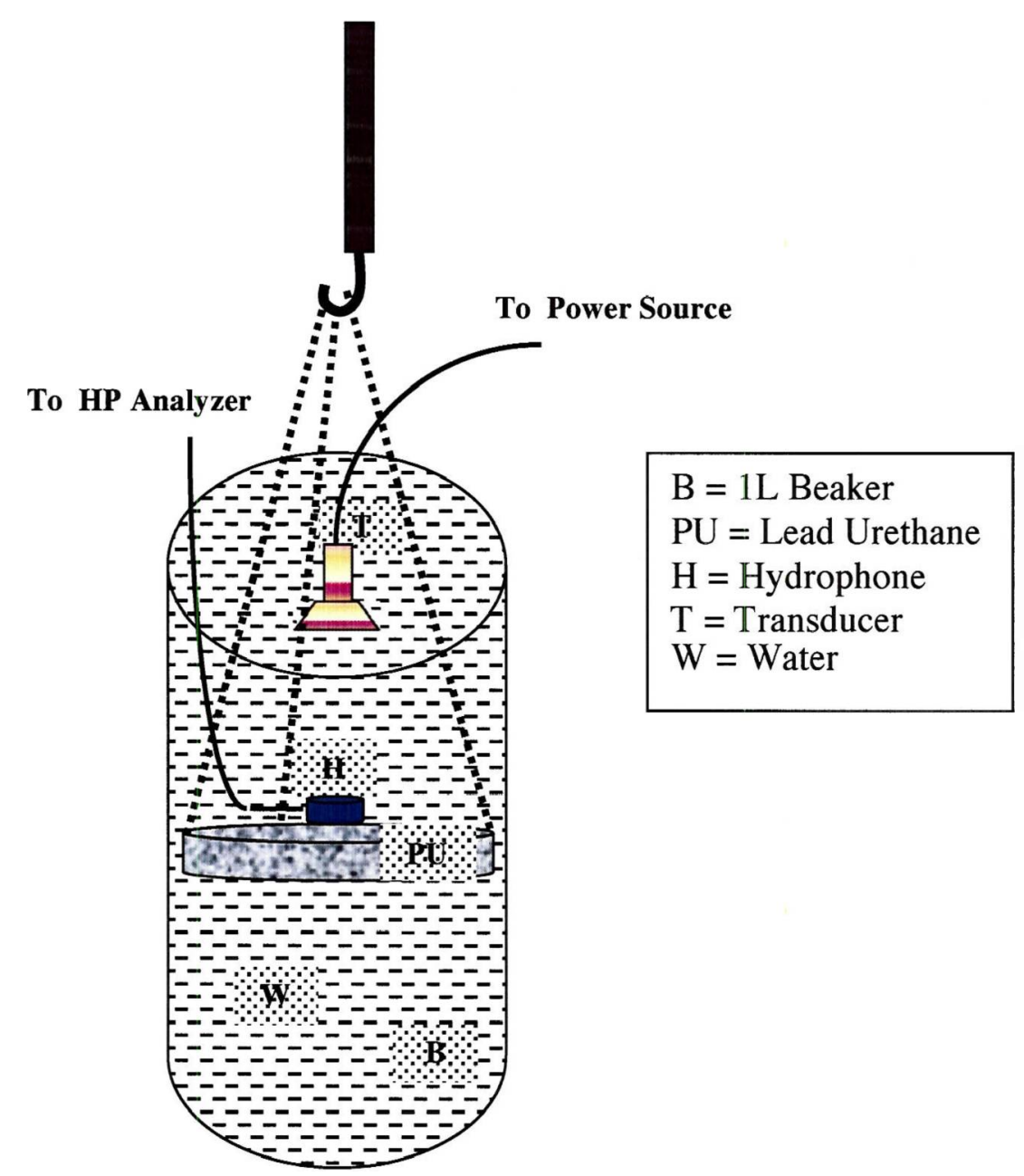

Figure 2.1.7 Experimental setup to elucidate sonic energy interaction with fluids

In general, the transducer-hydrophone separation did not have any effect of the measurement over the range $8-16 \mathrm{~cm}$. Using the hydrophone sensitivity curve and the measured receive drive level voltage, the sound pressure level (SPL) was calculated. To determine the onset of cavitation, the SPL is plotted as a function of the hydrophone drive level voltage. The break in the plot represents onset of cavitation.

Results and Discussion 
In order to determine the onset of cavitation, the SPL was plotted as a function of receive drive level voltage. A typical plot is shown in Figure 2.1.8. The results show that at a fixed frequency, the SPL increases linearly with receive drive level voltage with a break in the response observed at 35 drive level voltage. This break corresponds to the onset of cavitation when the transducer is operated at $20 \mathrm{kHz}$. These tests were repeated at different frequencies and the results for onset of cavitation are shown in Figure 2.1.9. In general, the drive level voltage threshold for onset of cavitation decreases with increasing frequency. As indicated earlier, our findings show that the voltage threshold for onset of cavitation is independent of transducer-hydrophone separation distance.

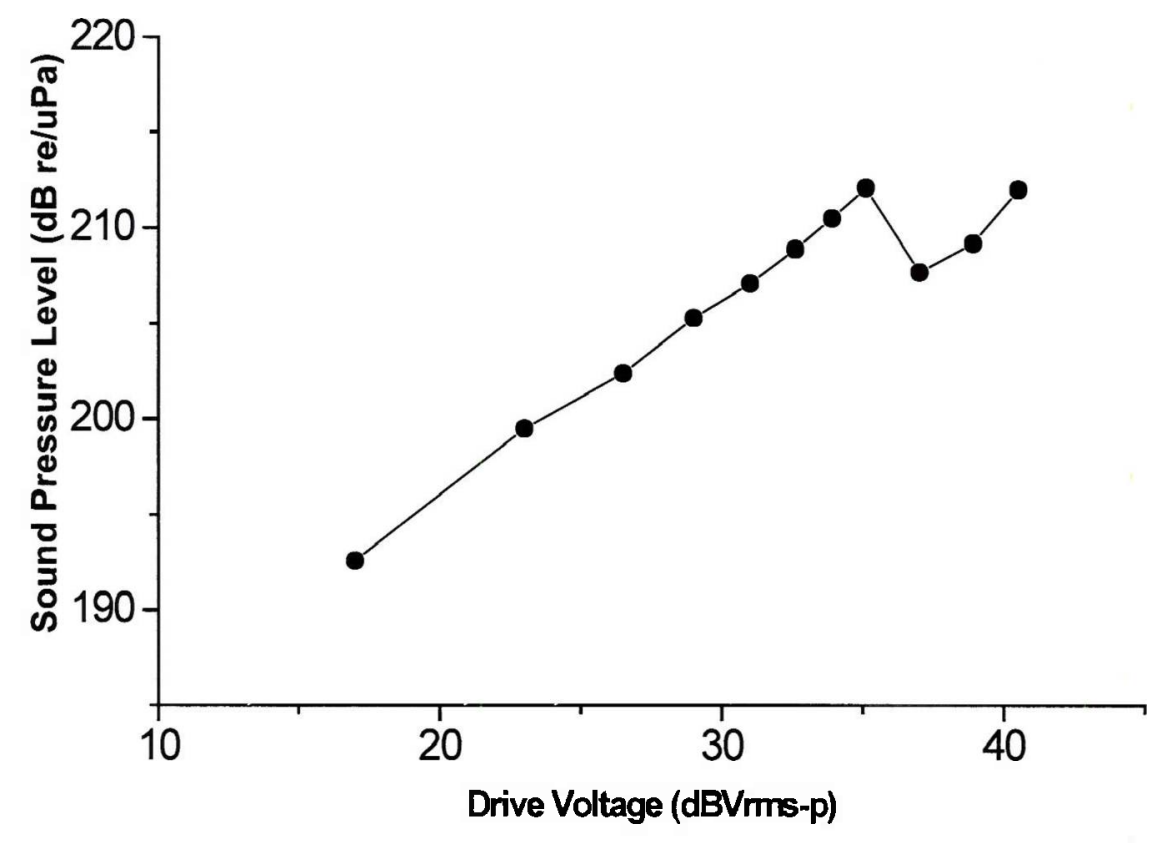

Figure 2.1.8 Linearity of pressure output with drive voltage, $20 \mathrm{kHz}, 0.004 \mathrm{sec}$ pulse length

\subsubsection{Power Output Under Cavitation}

\section{Procedure}

The experimental condition is similar that employed in determining the onset of cavitation with the exception that the power generator was set to $40 \%$ duty cycle and 
amplitude of $3.50 \mathrm{~V}$. Three hundred milliliters of tap water, initially at $18^{\circ} \mathrm{C}$, was put in a Styrofoam cup covered with a rubber disc. The Styrofoam cup was used to minimize heat losses to the surrounding. A digital thermocouple was inserted to record the temperature. The $20 \mathrm{kHz}$ transducer was also inserted through the disc and partially submerged in the water. To avoid temperature gradients, the water in the container was stirred throughout the test. Power supply to the transducer was increased until cavitation conditions were reached. The variation of temperature with time was recorded. In order to investigate the possible effect of nucleation sites on power dissipation under cavitation, two teaspoons of glass beads (60-120 mesh) were added to $300 \mathrm{~mL}$ of tap water initially at $18^{\circ} \mathrm{C}$ and the experiment repeated.

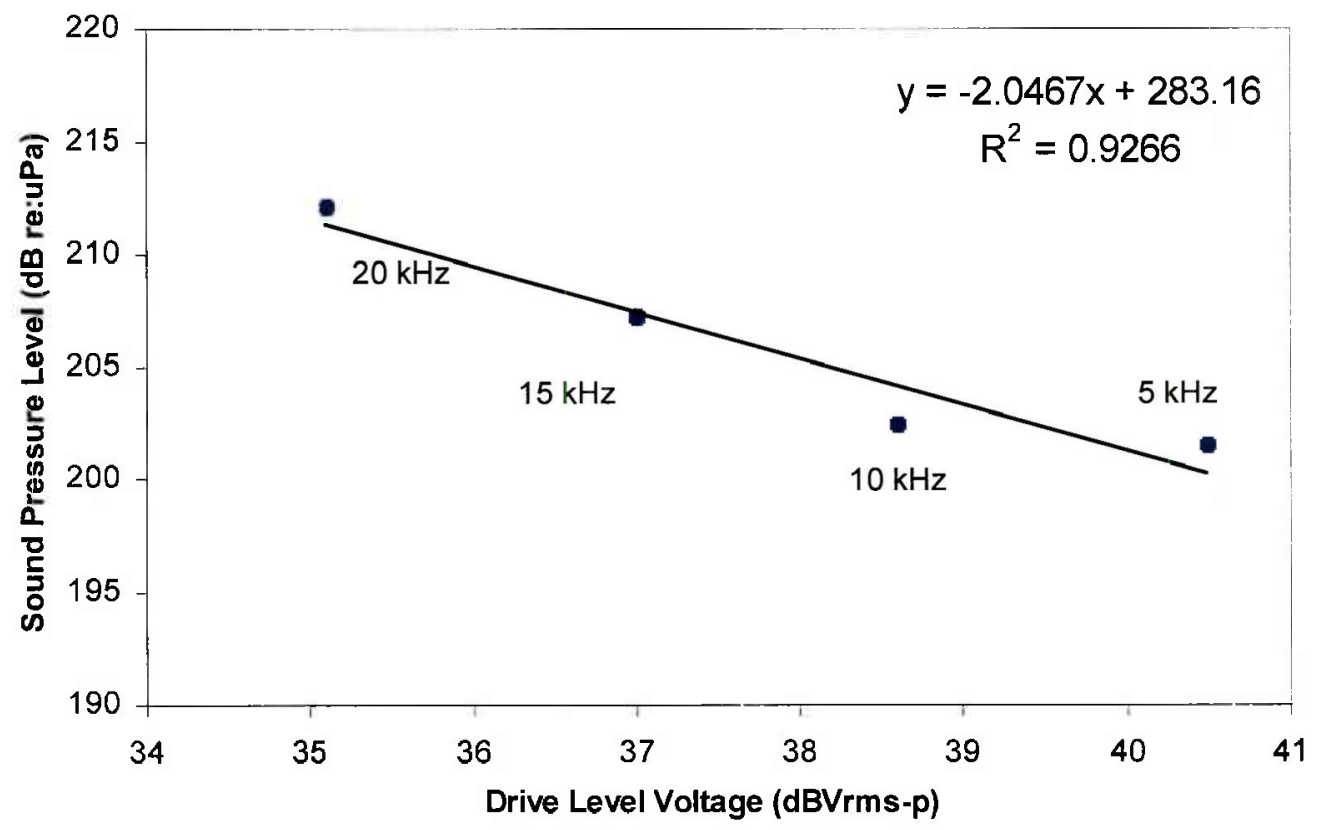

Figure 2.1.9 Cavitation threshold for different transducer frequencies

\section{Results and Discussion}

Figure 2.1.10 shows the variation of water temperature with time. A regression analysis of the data gave a slope of approximately $0.31^{\circ} \mathrm{C} / \mathrm{min}$. It can be shown from theory that: 


$$
P=M C_{p} \frac{d T}{d t}
$$

where $\mathrm{P}$ is the energy dissipation rate (watts), $\mathrm{M}, \mathrm{Cp}$ and $\mathrm{dT} / \mathrm{dt}$ are the mass and specific heat of water and slope of the plot, respectively. The results show that the presence of solid particles did not have a significant effect on the response. From the slope of the response and the mass of water, the energy dissipation rate is calculated to be $6.43 \mathrm{~W}$. Since the transducer has a diameter of $1 \mathrm{~cm}$, the specific energy dissipation is determined to be approximately $82 \mathrm{~kW} / \mathrm{m}^{2}$.

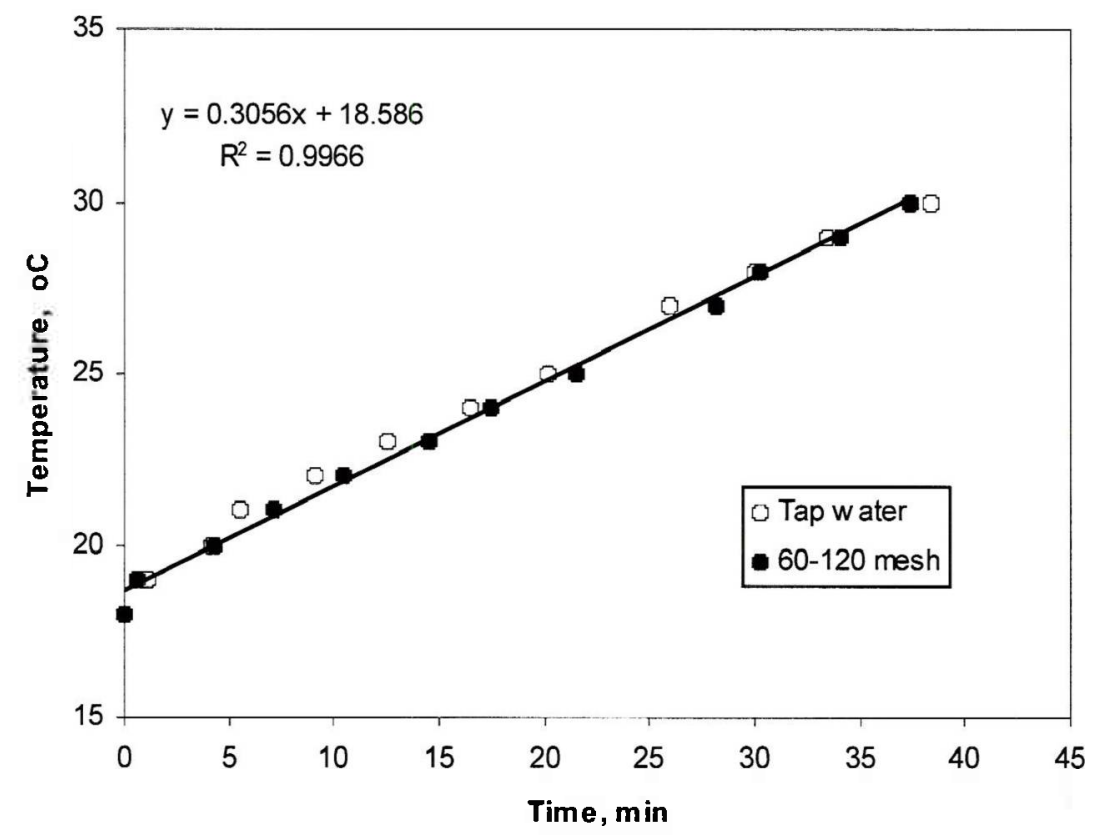

Figure 2.1.10 Variation of water temperature with time under cavitation conditions

It is obvious that the specific power dissipation rate was quite high even at such a low duty cycle. It is therefore possible that when deployed in a wellbore, the energy dissipation may promote the mobilization of deposited paraffins and waxes. Our laboratory experiment suggest the waxes are mobilized when the fluid temperature approaches $40^{\circ} \mathrm{C}$. Future experiments are planned to estimate the power dissipation at lower transducer operating frequencies. 


\subsubsection{Sonic Energy Interaction with Glass Beads Procedures}

About 200 and $550 \mathrm{~mL}$ of water were poured into 250 and $600 \mathrm{~mL}$ beakers, respectively. Glass beads (60-120 mesh size) were added to the beakers with gentle agitation. The distances from the transducer's head to the bottom of the beaker were approximately 6 and $12 \mathrm{~cm}$, respectively. The excited power was gradually increased until cavitation condition was attained.

\section{Results}

When the power was raised up to cavitation level, the glass beads spread out. The fine bubbles generated collapsed or coalesced to form larger bubbles, which were attached to the glass beads. After about 5 minutes, some of the attached bubbles rose to the surface where they burst releasing their load of particles, which dropped to the bottom of the beaker. When power was turned off, several agglomerated bubbles with glass beads rose to the surface as well. However, some glass beads and bubble conglomerates did not rise but stayed at the bottom where they remained after 14 days of observations. Since the glass beads are hydrophilic in nature, their recovery by the bubbles generated under cavitation conditions would suggest that the bubbles had a charge opposite that of the glass beads.

\subsubsection{Sonic Interaction with Oil} Procedure

One drop of used motor oil was dropped on a glass slide in air with the aid of a hypodermic syringe. The drop spread on the surface of the glass to a diameter of approximately $1 \mathrm{~cm}$. The slide was carefully placed on the hydrophone immersed in water and resting on the lead-urethane polymer block. The power to the transducer was increased until cavitation conditions were reached.

\section{Results}


Initially, the oil was observed to break into several small droplets which moved around the transducer near field, coalesced and/or rose to the surface. The small oil droplets behaved like rigid spheres. However, with continuous bombardment of the droplets, they coalesced into a big lump which remained intact even after sonic stimulation was turned off. A re-exposure of the glass slide to air resulted in an instantaneous spreading of the oil droplet. This observation would suggest that sonic excitation may promote oil coalescence by altering the oil interfacial tension. It is not clear at the present time if the behavior of the oil droplet is related to the release of certain components from the oil into the water. It was noted that during the excitation, a white fume-like cloud was released from the oil droplet into the water and was accompanied by a change in the physical appearance of the oil droplet (from a dark coloration to a grayish color). Additional experiments have been carried out using Pennsylvania paraffinic and other heavy crude oil sources with similar results.

Another experiment was conducted with the oil smeared on the surface of a 50 $\mathrm{mL}$ capacity volumetric glass container. The same phenomena occurring in the oil droplet on a glass slide were observed.

\subsubsection{Effect of Sonication on Properties of Crude Oil}

As noted above, we observed that white fumes were released into the water when the oil droplet was excited with an attendant change in the color of the droplet. We extended this study by using Pennsylvania paraffinic and Colorado heavy crude oil samples on glass slides and/or core samples. A digital video of the phenomena was produced and shown at the DOE-funded Stripper Well Consortium meeting held in Pittsburgh, Pennsylvania (November 12-13, 2002). The observation confirmed that under cavitation conditions, the oil initially breaks into smaller the oil droplets which later coalesce and migrate on the surface of the glass slide and/or core sample. Due to instability, the large oil droplets detach and rise to the surface of the water in the container.

We attempted to characterize the residue following sonic treatment. Specifically we wanted to determine if any components of the oil are susceptible to sonic treatment. This information is required to establish if sonic treatment will result in the production of 
oil with diminished or enhanced value - a factor that will impact the acceptability of the technology by oil producers. While we note that these tests were carried out in an environment where there was no confining pressure, it is nevertheless important to determine if gross changes in the oil properties results from the treatment. These tests will be repeated in the future using the slim-tube apparutus. We used a sample of Pennsylvania crude oil in this test.

\section{Procedure}

A sample of Pennsylvania crude oil was added to tap water and sealed in a plastic pipette-dropper. The pipette was put in water and irradiated with sonic energy. Power was increased until cavitation conditions were reached. The whole mixture turned milky with the application of sonic energy. At the end of the experiments, samples were kept undisturbed for several days. After the water was carefully removed, the oil residue in the tube was separated and prepared for GC-MS analysis.

A sample of oil residue was poured into a separatory funnel. Methylene chloride $\left(\mathrm{CH}_{2} \mathrm{Cl}_{2}\right)$ was added to dissolve and concentrate the organic components. The mixture was shaken vigorously and occasionally vented to relief pressure buildup. The methylene chloride was transferred into a column containing anhydrous magnesium sulfate to remove any traces of water from the sample. The dried sample (eluent from the column) was collected in a $2 \mathrm{~mL}$ bottle. The aqueous phase and a sample of untreated Pennsylvania crude were similarly prepared.

A Shimadzu Gas Chromatograph model GC-17A version 3 equipped with a Mass Spectrometer model QP-5000 and XTi-5 column was used for the chemical analysis. The column was $30 \mathrm{~cm}$ long, $0.25 \mathrm{~mm}$ ID with a 0.25 m film thickness. An auto sampler (model AOC-20i) was used to inject the samples into the GC-MS which was operated using Class 5000 software. The temperature of the column was initially programmed to increase from 40 to $150^{\circ} \mathrm{C}$ at the rate of $10^{\circ} \mathrm{C} /$ minutes. When the target temperature was reached, it was held there for 5 minutes. At the completion of this program, the temperature was gently raised from 150 to $300^{\circ} \mathrm{C}$ at a heating rate of $4^{\circ} \mathrm{C} /$ minutes. The column was maintained at $300^{\circ} \mathrm{C}$ for 10 minutes. The MS data collection time spanned 4 to 63.5 minutes and corresponded to 63.5 total acquisition period. For these analyses, we 
used a scan rate of 1000 counts/minute for mass to charge ratio $(\mathrm{m} / \mathrm{z})$ in the range of 40 to 300.

\section{Results and Discussion}

Figure 2.1.11 shows typical chromatograms of untreated Pennsylvania crude oil and the residue obtained after sonic treatment. The concentrations of the organics in the aqueous phase were low and as such will not be discussed at this time. Efforts are underway to concentrate these species and the results will be reported at a later date. For the identification and integration of the peak areas, we used a gradient that was $1 \%$ of the maximum intensity observed for the scan. We used the Class 5000 software to match and identify the components present in the different sample. Tables 2.1.3 and 2.1.4 show the results from this match.

Our analysis confirms that the predominant species in the untreated crude oil were paraffins (mostly straight chain alkanes) with smaller quantities of branched alkanes and aromatic compounds. While the composition of the residue from the sonic treated samples was similar to that of the untreated crude oil, there were several differences. For example, the lighter fractions with low retention times were lost during the treatment as were the aromatic and branched alkanes. Since the aromatic components are not needed in the production of lubricants or gasoline fractions, their loss may be desirable. We note that in tests using the sealed pipette droppers, the tube appeared to be under vacuum. This would be indicative of the possible reaction of the oxygen trapped in the tube with the aromatic fraction. This observation would suggest that sonication may be a viable procedure for the treatment of organic wastes containing aromatic fractions.

\subsubsection{Effect of Sonic Stimulation on Permeability Experimental Set-Up}

Figure 2.1.12 shows a schematic representation of the experimental setup used to study the effect of acoustical stimulation on the permeability of a Berea sandstone core sample. A combination gas and liquid permeamter (Temco model GP/LP - 401 L) 


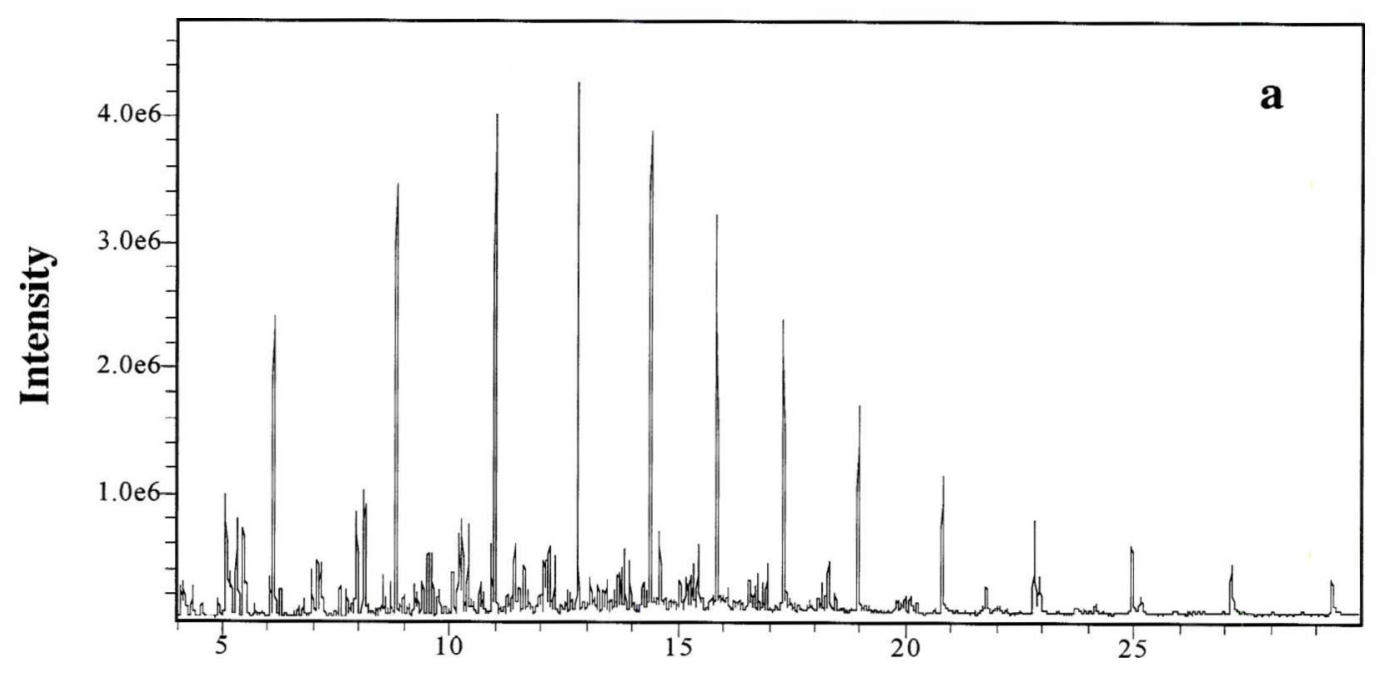

\section{Retention time (min)}

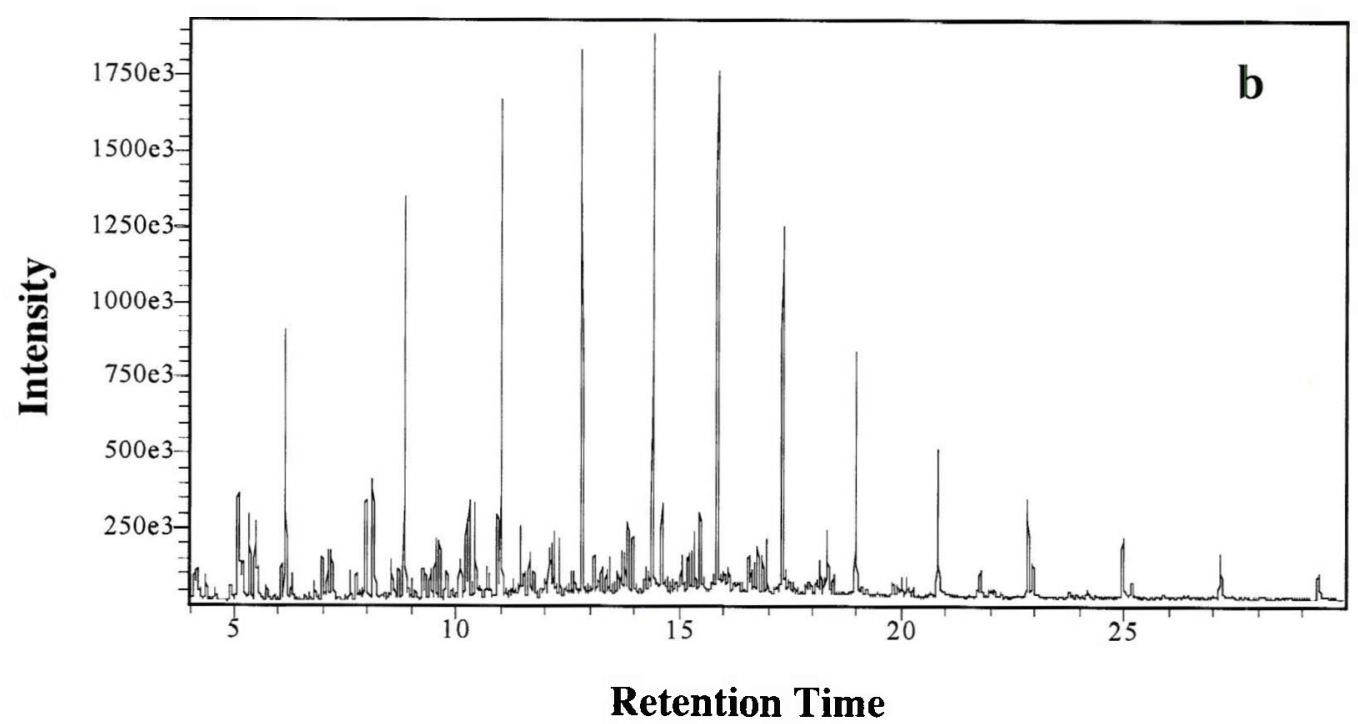

Figure 2.1.11 Total ion chromatograms of (a) untreated PA crude oil and (b) Pa crude after irradiated with sonic energy 
Table 2.1.3 Qualitative analysis of untreated PA crude oil by total ion chromatography

\begin{tabular}{|c|c|c|c|l|}
\hline $\begin{array}{r}\text { Retention } \\
\text { Time } \\
(\mathbf{m i n})\end{array}$ & $\begin{array}{r}\text { Area } \\
\%\end{array}$ & Formula & MW & Compound \\
\hline 5.305 & 2.95 & $\mathrm{C}_{8} \mathrm{H}_{18}$ & 114 & 3-Methyheptane \\
\hline 6.121 & 9.92 & $\mathrm{C}_{8} \mathrm{H}_{18}$ & 114 & Octane \\
\hline 7.925 & 3.13 & $\mathrm{C}_{9} \mathrm{H}_{20}$ & 128 & 2,4-Dimethylheptane \\
\hline 8.086 & 6.26 & $\mathrm{C}_{8} \mathrm{H}_{10}$ & 106 & 1,3-Dimethylbenzene \\
\hline 8.825 & 11.42 & $\mathrm{C}_{9} \mathrm{H}_{20}$ & 128 & Nonane \\
\hline 10.270 & 2.26 & $\mathrm{C}_{10} \mathrm{H}_{22}$ & 142 & 2-Methylnonane \\
\hline 10.907 & 2.11 & $\mathrm{C}_{9} \mathrm{H}_{12}$ & 120 & 1,2,4-Trimethylbenzene \\
\hline 10.992 & 11.81 & $\mathrm{C}_{11} \mathrm{H}_{24}$ & 156 & Undecane \\
\hline 12.805 & 12.35 & $\mathrm{C}_{11} \mathrm{H}_{24}$ & 156 & Undecane \\
\hline 14.401 & 10.81 & $\mathrm{C}_{11} \mathrm{H}_{24}$ & 156 & Undecane \\
\hline 15.853 & 9.22 & $\mathrm{C}_{11} \mathrm{H}_{24}$ & 156 & Undecane \\
\hline 17.315 & 7.37 & $\mathrm{C}_{14} \mathrm{H}_{30}$ & 198 & Tetradecane \\
\hline 18.958 & 6.06 & $\mathrm{C}_{14} \mathrm{H}_{30}$ & 198 & Tetradecane \\
\hline 20.800 & 4.33 & $\mathrm{C}_{14} \mathrm{H}_{30}$ & 198 & Tetradecane \\
\hline
\end{tabular}

Table 2.1.4 Qualitative analysis of residue from sonic treated PA crude oil by total ion chromatography

\begin{tabular}{|c|c|l|l|l|}
\hline $\begin{array}{c}\text { Retention } \\
\text { Time } \\
(\text { min) }\end{array}$ & $\begin{array}{c}\text { Area } \\
\%\end{array}$ & Formula & MW & Compound \\
\hline 6.139 & 10.02 & $\mathrm{C}_{8} \mathrm{H}_{18}$ & 114 & Octane \\
\hline 8.840 & 12.14 & $\mathrm{C}_{9} \mathrm{H}_{20}$ & 128 & Nonane \\
\hline 11.005 & 13.10 & $\mathrm{C}_{9} \mathrm{H}_{20}$ & 128 & Nonane \\
\hline 12.817 & 14.74 & $\mathrm{C}_{11} \mathrm{H}_{24}$ & 156 & Undecane \\
\hline 14.414 & 13.96 & $\mathrm{C}_{11} \mathrm{H}_{24}$ & 156 & Undecane \\
\hline 15.867 & 12.62 & $\mathrm{C}_{11} \mathrm{H}_{24}$ & 156 & Undecane \\
\hline 17.329 & 10.19 & $\mathrm{C}_{14} \mathrm{H}_{30}$ & 198 & Tetradecane \\
\hline 18.973 & 8.04 & $\mathrm{C}_{14} \mathrm{H}_{30}$ & 198 & Tetradecane \\
\hline 20.818 & 5.19 & $\mathrm{C}_{14} \mathrm{H}_{30}$ & 198 & Tetradecane \\
\hline
\end{tabular}

was used to measure the liquid permeability. A Berea sandstone core sample 1.50 inches $(3.81 \mathrm{~cm})$ in diameter and 3.0 inches $(7.62 \mathrm{~cm})$ long was held in a rubber sleeve placed in the Temco core holder (model RCHR 1-1.5). A retainer and a distribution plug at one end of the core holder were modified to accommodate an ultrasonic Branson Horn tip (model 101-147-037) placed at the fluid inlet face of the core. A 250 watts Branson converter (model 101-135-022) was used to excite the horn at a fixed frequency of $20 \mathrm{kHz}$. The set- 


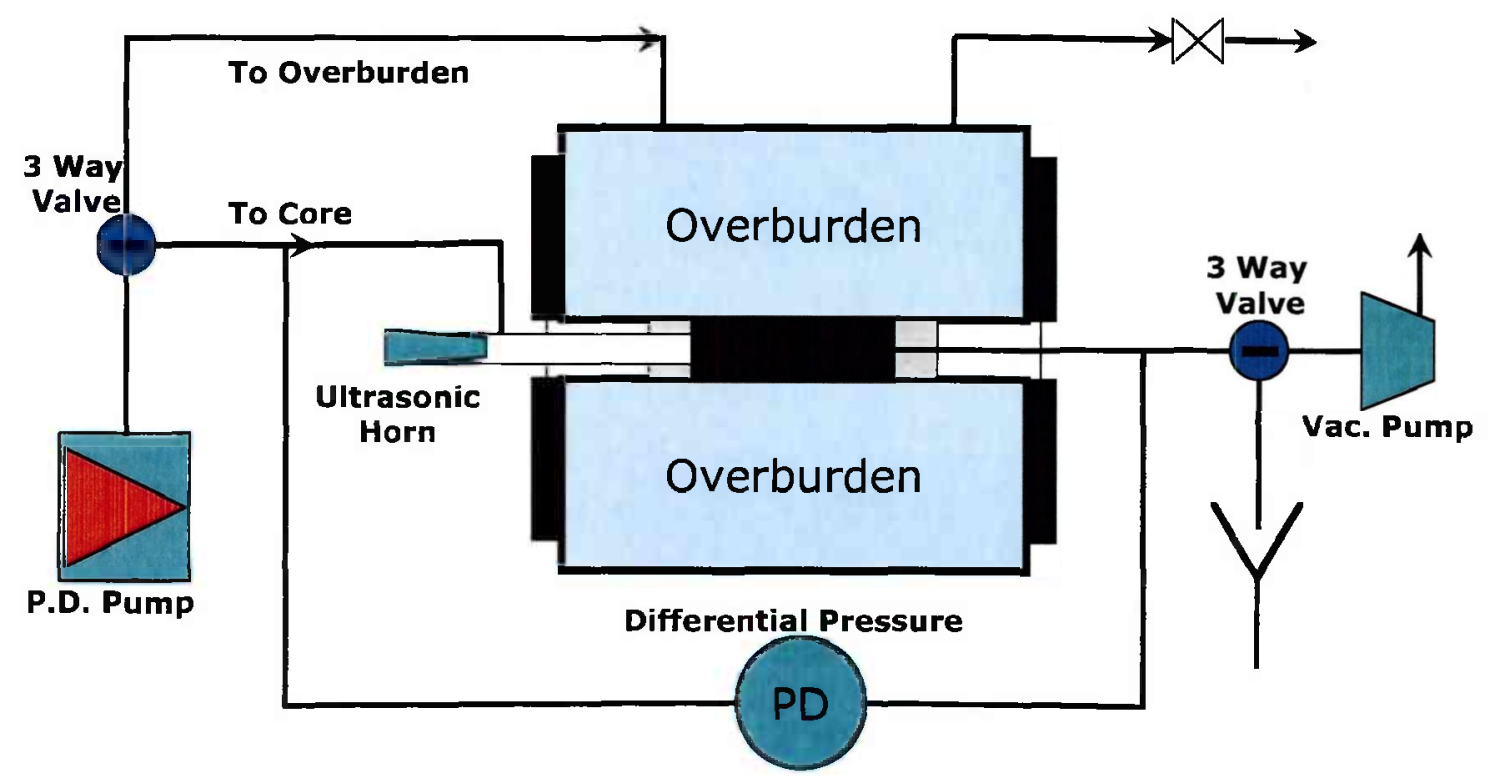

Figure 2.1.12 Schematic of the experimental set-up

up includes a vacuum pump to evacuate air from the core sample and a thermocouple located a few centimeters from the tip of the horn to measure the temperature of the water. By adjusting the output control setting and the duty cycle, the excitation power was varied. A high pressure pump (Edwards Model ED 50) was used to provide a radial confining pressure of 900 psig.

\section{Experimental Procedure}

A Berea sandstone core sample (labeled B-1) was dried at $60^{\circ} \mathrm{C}$ in a Fisher Isotemp Oven (model 496). The original weight of the sample was $184.67 \mathrm{~g}$. After one hour of drying there was no significant change in the sample weight (184.27 g). After cooling the sample it was placed in the core holder and the vacuum pump turned on. During the evacuation of the air, a vacuum pressure of -28 inches of $\mathrm{Hg}$ was attained. The core was flushed with high purity de-ionized water while the vacuum pump was still running. Flushing of the core was terminated when water started dripping into the liquid separator at the inlet of the vacuum pump. With the high confining pressure, it is reasonable to assume that the flow of fluid was in the axial direction.

Prior to the sonication experiments, the permeability of the sample was determined. The water flow rate for all the measurements reported here was fixed at 0.1 $\mathrm{mL} / \mathrm{min}$. With this low flow rate, laminar flow conditions prevail. The temperature of the 
water that passed through the core, overburden or confining pressure and differential pressure were automatically monitored using a SmartPerm-GLL version 1.0 software. From the differential pressure across the core and the measured temperature of the fluid, the permeability of the sample was instantaneously calculated and displayed on the computer screen. Data logging during the permeability measurement was automatic and sampling frequency could be adjusted as needed. For the present study, the pressure, temperature and computed permeability readings were recorded every minute. When the pre-sonication permeability was constant, the sonic tool was turned and the process repeated. For the present study, the duty cycle was fixed at $10 \%$ while the output setting was varied from 1 to 4 (on a scale of 1-10).

\section{Results and Discussion}

Figure 2.1.13 shows the permeability of the core sample as a function of time for the pre-sonication experiment. The duration of the test was $4 \mathrm{~h}$ and the total volume of water passed through the core was approximately 1.3 pore volumes (assuming a core porosity of $20 \%$ ). It is evident that the permeability of the sample attained a steady value of about $35 \mathrm{md}$ after about 30 minutes.

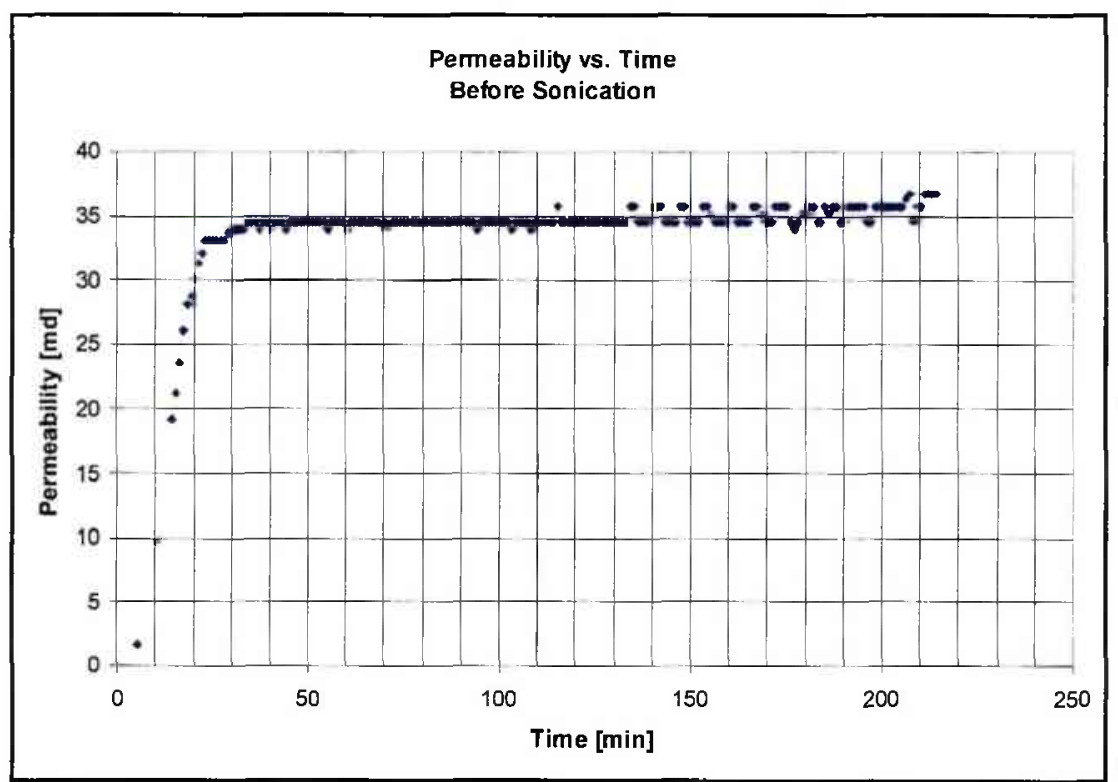

Figure 2.1.13 Pre-sonication permeability-time plot 
Following the attainment of steady permeability values, the sonic tool was turned on to the lowest power output setting (10\% duty cycle and 8 watts). Figure 2.1 .14 shows the variation of permeability with duration of sonic application. The results show a steep increase in the permeability values with sonic energy application. After about 20 minutes, the values appear to stabilize in the range of 55-58 $\mathrm{md}$. Using this lower value, our findings show that the application of the sonic energy produced at least a $57 \%$ increase in the permeability of the core. It is noted that during the test, the temperature of the water did not change by more than $1^{\circ} \mathrm{C}$, so the effect observed here cannot be attributed to viscosity changes (Poesio et al., 2002). Furthermore, even if the temperature changes, the software has in-built capabilities to compensate for the viscosity variation with temperature.

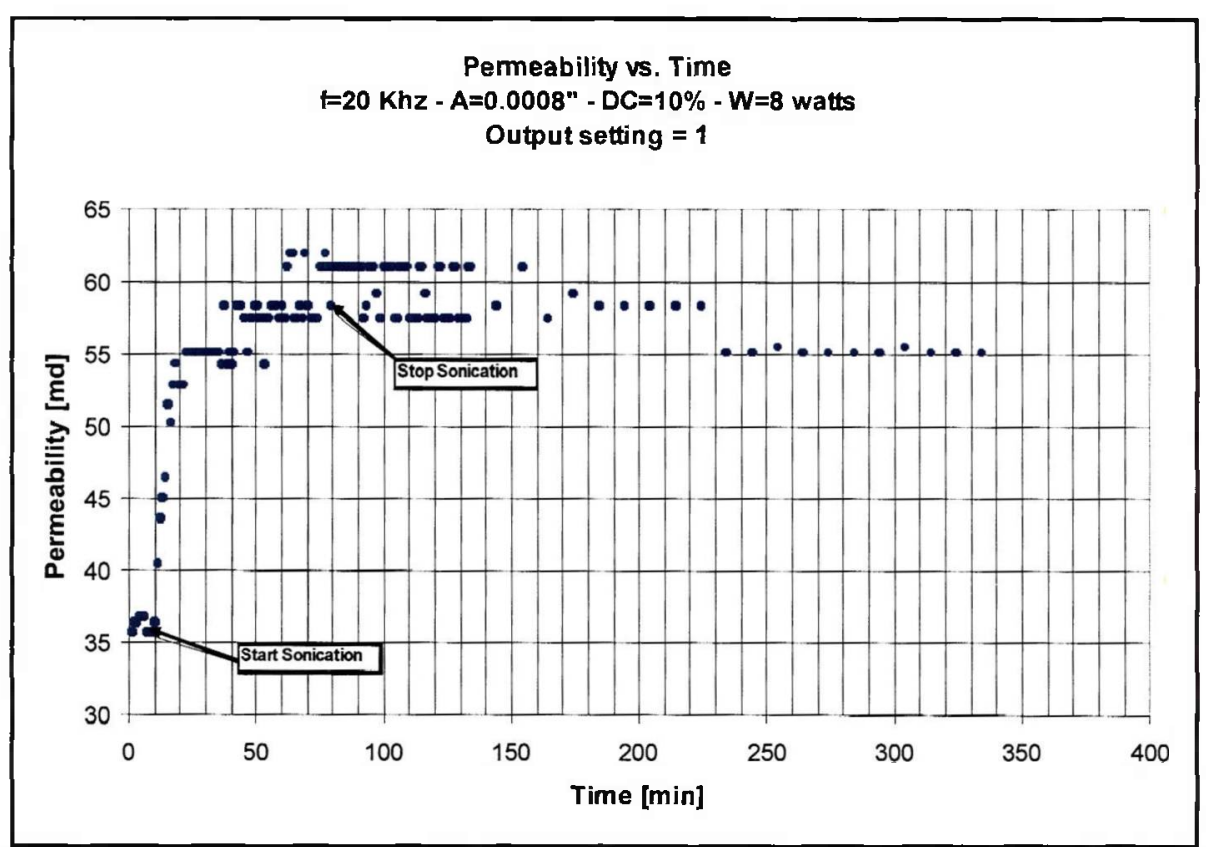

Figure 2.1.14 Permeability-time plot at $10 \%$ duty cycle and output setting of 1

Given the encouraging results, it was decided to establish the permanency of the observed effect. Thus, after about $1 \mathrm{~h}$ of sonic stimulation, the tool was turned off while the liquid flow was maintained. The results show initial oscillations occurred before assuming a constant value of about $56 \mathrm{md}$. Since the post-sonication permeability value is in the same range as that observed during sonication, it may be concluded that the change is indeed permanent in nature. 
Given the excellent results observed, it was decided to evaluate the effect of output power level on the observed permeability of the core. The core used was kept overnight in the core holder and the permeability determined the next day prior to the start of sonication. Figure 2.1.15 shows the variation of the liquid permeability with time. The pre-sonication values again confirm the permanency of the changes in the permeability value. With increasing power, there was a further modest increase in the permeability value of the core which was again maintained after the tool was turned off (see Figure 2.1.15). Permeability measurements were also determined at output power settings of 3 and 4 and the results are summarized in Table 2.1.5. Our results confirm that the application of sonic energy results in over $100 \%$ increase in the permeability of the core sample.

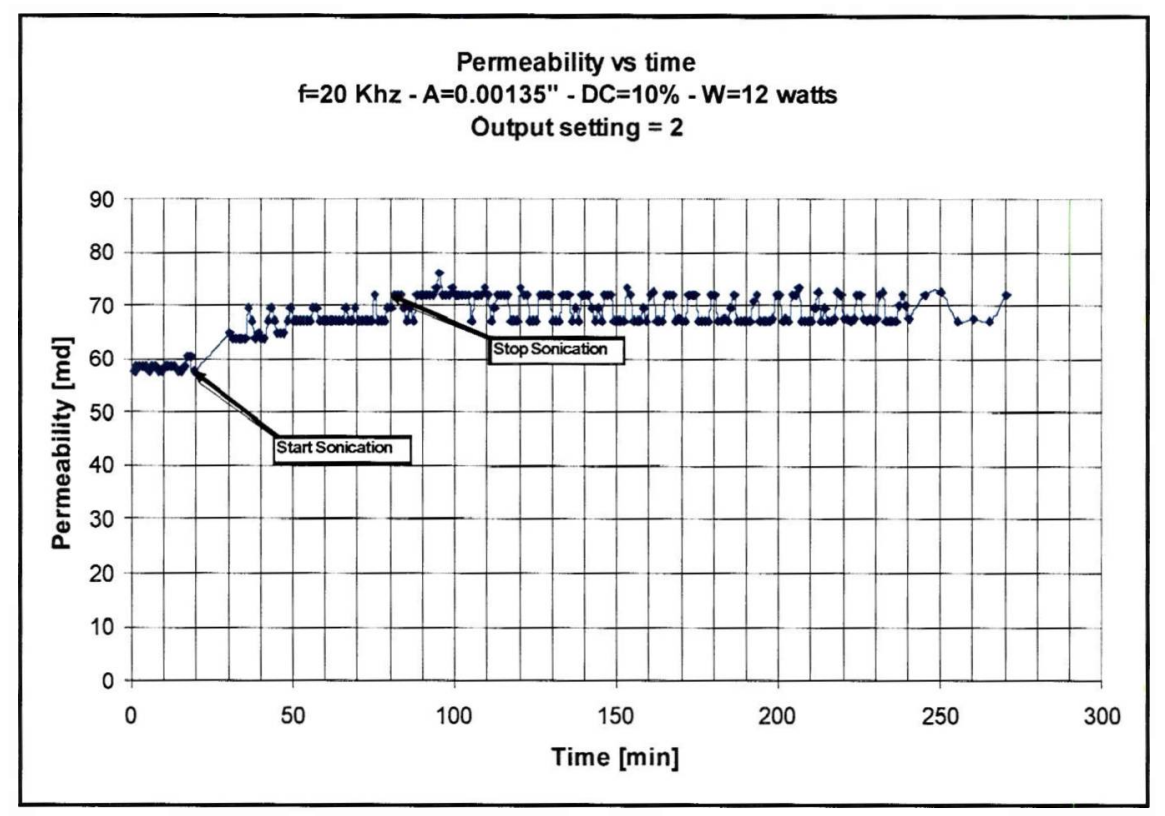

Figure 2.1.15 Permeability-time plot at output setting $=2$, duty cycle $=10 \%$, frequency $=20 \mathrm{kHz}$ and corresponding power of 12 watts

During the sonication experiments, there was a consistent increase in the overburden pressure, which dissipated when the sonic tool was turned off (see Figure 2.1.16). While an increase in the temperature of the confining fluid may partially explain the rise in overburden pressure, we do not consider this to be the case since the rubber 
sleeve is expected to act as an insulator. Attempts will be made in the future to determine the reasons for the increase in confining pressure.

Table 2.1.5 Summary of sonic energy effect on permeability of Berea Sandstone

\begin{tabular}{|c|c|c|}
\hline $\begin{array}{c}\text { Output Power } \\
\text { setting }\end{array}$ & Permeability, md & $\begin{array}{c}\text { Permeability } \\
\text { increase, } \%^{*}\end{array}$ \\
\hline & & 0 \\
\hline 0 & 35 & 57 \\
\hline 1 & 55 & 100 \\
\hline 2 & 70 & 126 \\
\hline 3 & 79 & 134 \\
\hline 4 & 82 & \\
\hline
\end{tabular}

* Relative to the value at 0 setting

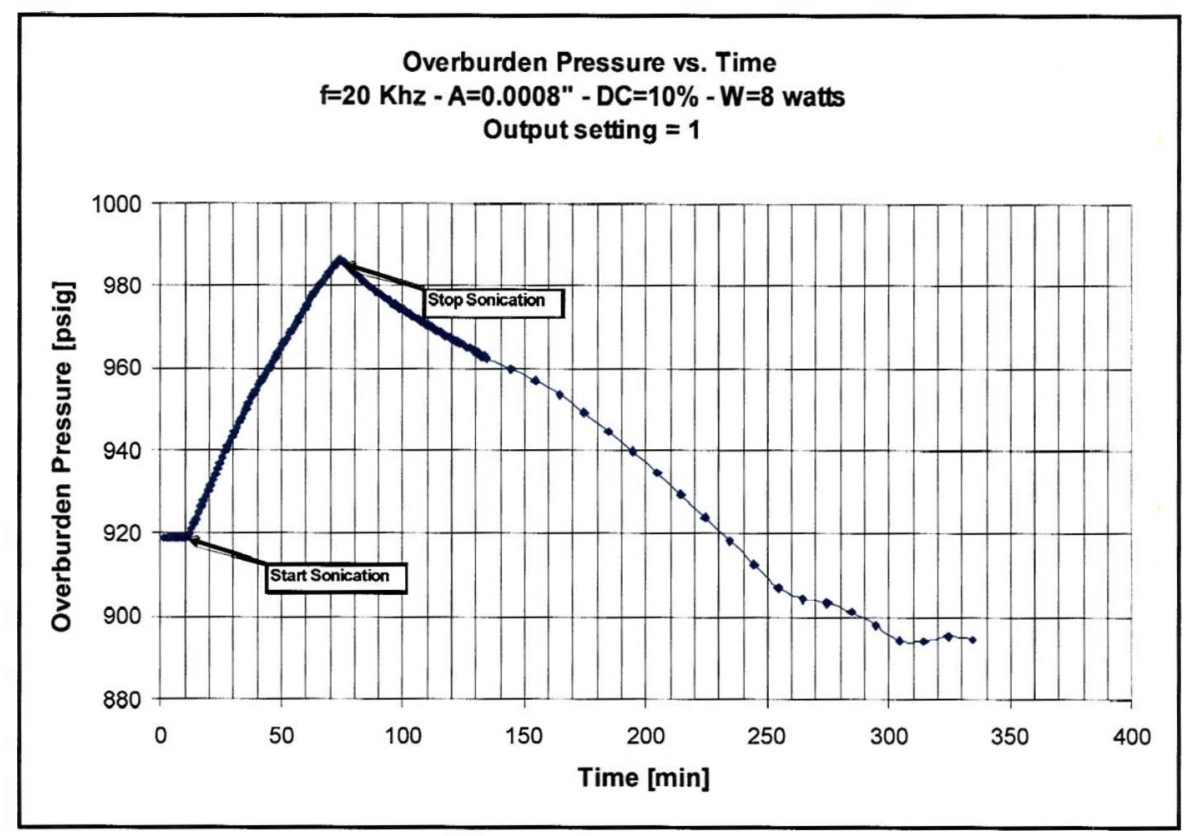

Figure 2.1.16 Overburden pressure-time plot at output setting $=1$, duty cycle $=10 \%$, frequency $=20 \mathrm{kHz}$ and corresponding power of 8 watts

\section{Conclusions}

The main objective of this experiment was to try to identify the mechanisms governing permeability enhancement during the application of the sonic energy. 
There are two possible mechanisms that may account for the increase in the effective permeability of the core. They are acoustic streaming and acoustic cavitation.

Acoustic streaming is supposed to create a pressure gradient capable of helping to move the fluid across the core. If streaming is the driving force it should be observed at low power intensities, and the pressure drop across the core should decrease without a significant increase in temperature of the fluid. On the other hand, if cavitation is the governing mechanism, the implosion of the bubbles should create an evident increase in temperature. The violent nature of this phenomenon should be reflected in a particular noise.

The results obtained in this experiment clearly suggest that acoustic streaming is the governing mechanism for the fluid flow enhancement. Most of the effective permeability enhancement occurred at low power application, where no increase in temperature was detected. As power was increased, the efficiency of the stimulation method decreased dramatically. Most of the energy was used for generating heat that had no significant impact on flow enhancement. 


\subsubsection{Theoretical Development of Thermal Profile during Sonication}

In the case of sonic stimulation, there will be heat dissipation resulting in a temperature rise in the system. Temperature distribution in a core sample undergoing sonic stimulation was estimated numerically. The temperature profile was determined as a function of the core length. Identifying the temperature profile is important in determining whether the temperature rise could affect the mobility and/or phase distribution of paraffin, waxes or other substances that may be present in the sample. In the present study, the temperature profile was determined for an undamaged Berea sandstone sample. Since the sample was surrounded by a pressurized rubber sleeve one dimensional fluid flow was established in the system. Description of the model, assumptions, numerical methods, and the discussion of the results was presented by Ozdemir (2004) as part of this research project.

\subsubsection{Remediation of Damaged Cores using Sonication}

The effectiveness of ultrasonic waves in removing wellbore damage was investigated at the laboratory scale. Berea sandstone samples were first damaged by injecting fresh water and the damaged cores were subjected to sonic stimulation with and without a solvent. Acoustic energy was applied to fired and unfired core samples in both co-current and counter-current flow directions while ethanol was used as the solvent. Liquid permeability of the cores was monitored as a function of time before, during and after the application of acoustic energy and Klinkenberg effect was determined before and after the damage. The results showed that sonic stimulation was more effective in co-current direction and the combination of sonic stimulation with the solvent did not create a significant improvement in the permeability value.

The results of this experimental study can be summarized as follows: 
1) Fresh water resulted in damage to Berea sandstone samples. The application of sonic energy produced an increase in the permeability which did not appear to be a function of power output level.

2) Although surface tension does not appear in Darcy equation it has an effect on the permeability of the rock. Our results show that the measured liquid permeability increases with ethanol concentration. Since the liquid surface tension decreases with increasing ethanol concentration, it appears that measured liquid permeability values may depend on the solvent used. This effect may be tied to wetting properties of the rock.

3) With fired and dried core samples, it appears that sonication in co-current flow direction gave higher permeability values when compared with the counter-current flow arrangement.

4) With fresh water flow, higher permeability values were obtained with fired cores, thus confirming that firing reduced the swelling potential of the Berea sandstones.

5) The results suggest that once the core is subjected to sonic stimulation additional application of sonic energy and a use of a higher power output did not have any significant effect in the permeability of the sample. Details on the experiments conducted and the results obtained can be obtained in the work of Ozdemir (2004). This work was part of this research project. 


\subsection{Task 2. Development of a Computer Model for Scale-Up}

\subsubsection{Computer Model for Scale-Up}

\subsubsection{Objective}

Previous studies have shown that excitation of the reservoir with sonic energy (akin to external cyclic pressure loading of reservoirs) can sometimes have the desirable effects of increased recovery and yet other times little or no effect. The objective of this study is to investigate the possibility enhancing production from marginal reservoirs by using transducers to introduce the cyclic pressure fluctuations in the form of acoustic energy into the region surrounding the wellbore. The objective set out in Task 2 is to build a numerical model to simulate laboratory experiments designed to capture the phenomena of enhanced production due to cyclic loading as well as isolate the factors contributing to that enhancement production. Once that is achieved it is envisioned that the window of opportunity presented by the cyclic pressure loading on reservoir production can be delineated so that field scale transducer can be designed and built. The model can then be used for scale-up purposes in the field.

\subsubsection{Methodology}

To achieve the objectives in Task 2, we have set out the following tactical approach to the problem at hand.

1. Derivation of the general equations governing fluid flow;

2. Adaptation of these equations to the porous media;

3. Replication of results from laboratory experiments;

4. Isolation of contributing phenomena using derived terms;

5. Association between derived and porous media characterization terms;

6. Establishing effect on porous media characterization by association; and

7. Designing the scaled up model.

In this report we will present both sets of governing equations. This will be followed by a discussion on the methodology designed to achieve our modeling objective. The numerical partial differential equation solver built for this purposed will be presented along with the validation runs. Finally some modeling results of simplified experiments will be presented. 


\subsection{The General Transport Equation}

The transport of any extensive property $E$ in the phase is governed by the following equation at the microscopic scale:

$$
\frac{\partial e}{\partial t}=-\nabla \cdot\left(e V+J^{E}\right)+\rho \Gamma^{E}
$$

Where

$$
\begin{aligned}
& e=\text { Density of } E \\
& V=\text { Velocity vector } \\
& J^{E}=\text { Diffusive flux with respect to } V \\
& \rho=\text { Mass density of phase } \\
& \Gamma^{E}=\text { Net rate of production of } E \text { within the phase }
\end{aligned}
$$

The term density in reference to the symbol e denotes the amount of the extensive property $\mathrm{E}$ that is present in the phase, $\mathrm{V}$, and $\mathrm{J}$.

On a microscopic scale, this equation reads simply as a balance of the extensive property $\mathrm{E}$ in finite domain with the temporal derivative being the rate of accumulation of that property with respect to time, and the spatial derivative describing the evolution of the flux of the property as it is being transported by the phase through space, whereas the last term in Equation 2.1 represents the source/sink term required to account for the generation and loss of property $\mathrm{E}$ in the finite domain. Equation 2.1 it is particularly well suited for tracking conserved properties such as mass, momentum and energy, which are essential components of any fluid dynamic modeling endeavor.

\subsection{Representative Elementary Volume (REV)}

However the complexity and size of the domains that are of interest in modeling of the porous media prohibit the practical usage of this fundamental equation in the form above. The problem has its roots in the basis of numerical modeling, where in the interest of minimizing the computational workload an "appropriate" amount of accuracy must be sacrificed. To achieve this matter is assumed to exist as a continuum where the 
randomness of events at a molecular level is replaced by more statistically representative volumes where events are indeed measurable.

For example, the extensive property mass, at a molecular level, is a random property depending on whether the molecule exists or not in the space where the observer chooses to look. However, over larger volumes such as a cubic meter, this randomness is lost since the inclusion or omission of a few million more molecules becomes statistically insignificant to the total mass of molecules enclosed in the cubic meter. This leads to the term mass density that is statistically representative of that volume. However, it is clear that the size of the elementary volume that is representative is dependent on the homogeneity of the molecules in that volume as well as the scale of the events being modeled.

This makes the task of choosing the representative elementary volume (REV) more problematic when considering fluid motion in the porous media where the ability of molecules to be transported between two points is complicated by the existence of a solid phase that may be impervious to the fluid molecules causing them to exist and travel within the pore spaces and interconnecting channels. Due to the complex sedimentation processes that govern the distribution and configuration of the pore spaces and their interconnecting channels, the choice of finite volumes that can be assumed to have homogeneous properties becomes exceedingly small making the simulation task impractical as a result of the huge computation overhead required for modeling the transportation processing. In such cases, the representative capability of the volume is expanded to include heterogeneity at a scale that is chosen to fulfill the constraint of "appropriate" accuracy. This is achieved by averaging the extensive properties of many homogeneous elementary volumes to a single value that is representative from a statistical point of view. The averaged extensive properties of these significantly larger REV can now be used in similarly averaged equations of the form of Equation 2.1.

\subsection{The Averaged General Transport Equation (GTE)}

To transform the averaged general transport equation (GTE) presented above, a set of averaging rules are needed. Examination of the GTE makes it clear that we must establish rules to average several mathematical concepts, ranging from simple addition to 
complex derivatives taken over a moving control volume. The averaging rules will be presented as the need for them arises with detailed derivations found in Appendix B.1. In this report, the average quantity will be differentiated from an un-averaged value by a bar over the averaged property.

Now we are in a position to take the average of Equation 2.1 giving

$$
\frac{\partial e}{\partial t}=-\nabla \cdot\left(e V+J^{E}\right)+\rho \Gamma^{E} \rightarrow \frac{\overline{\partial e}}{\partial t}=\overline{-\nabla \cdot\left(e V+J^{E}\right)+\rho \Gamma^{E}}
$$

In order to proceed from this point, we need to elaborate on the mathematical concept of averaging as it pertains to the porous media. In Figure 2.2.1, the continua of the phases that make up a REV in the porous media are shown schematically. Figure 2.2.1 shows two phases $\alpha$ and $\beta$, and three separating interfaces representing the surfaces in the REV. The surface between the two phases within the same REV is denoted by $S_{\alpha \beta}$; and surfaces separating the $\alpha$ and $\beta$ phases in two adjacent REVs are denoted as $S_{\alpha \alpha}$ and $S_{\beta \beta}$, respectively. The average of a property in this REV can be defined in two separate ways as the following discussion illustrates.

In the first definition, the average of phasial property taken over the REV is

$$
\overline{e(x, t)}=\frac{1}{U_{R E V}} \int_{U_{R E V}} e(x, t) \gamma_{\alpha}(x, t) d U_{R E V}
$$

where the function $\gamma_{\alpha}(x, t)$ takes the value of unity when the vector $\mathrm{x}$ points to a location within the $\alpha$ phase and zero when the vector points to a location elsewhere in the REV. 


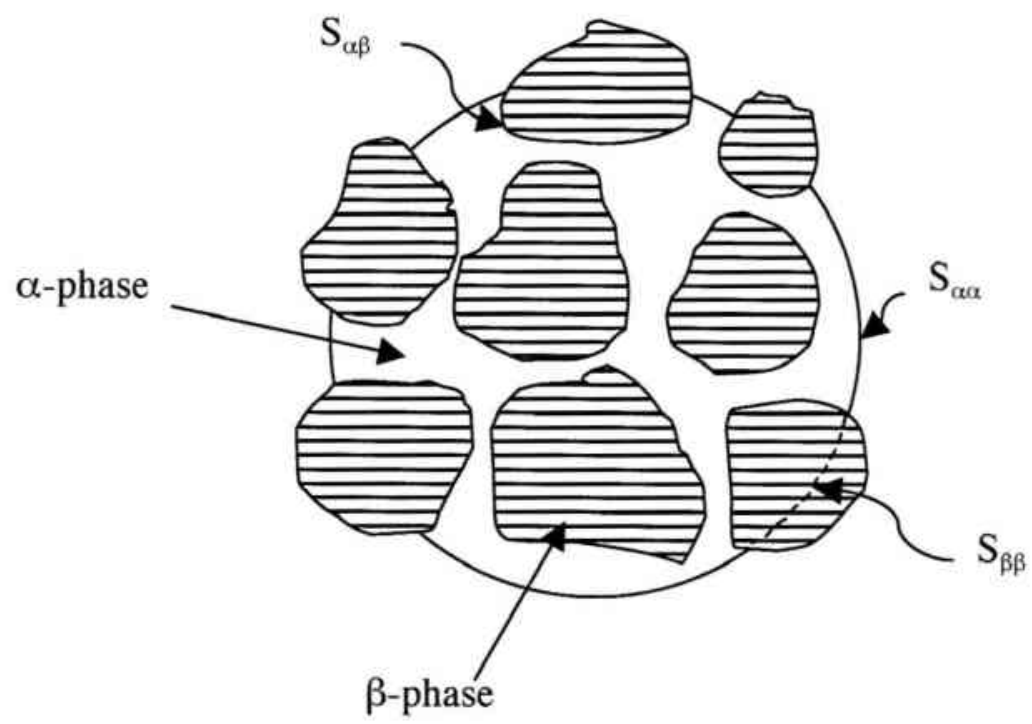

Figure 2.2.1. Schematic of a REV showing the interfacial surfaces between the phases

Alternatively, in the second definition, the average of the phasial property is taken over the volume of the phase:

$$
\overline{e(x, t)}^{\alpha}=\frac{1}{U_{\alpha}} \int_{U_{\alpha}} e(x, t) d U_{\alpha}
$$

Here, since we are taking the average over the only the phase $\alpha$ in the REV, the the function is not needed to isolate position vectors within the phase $\alpha$.

From these two averages of phasial properties, we can establish a relationship between averages taken over the whole control volume and those taken over only the phase of interest:

$$
\frac{\overline{e(x, t)}}{\overline{e(x, t)}}=\frac{U_{\alpha}}{U_{R E V}} \frac{\int_{U_{R E V}} e(x, t) \gamma_{\alpha}(x, t) d U_{R E V}}{\int_{U_{\alpha}} e(x, t) d U_{\alpha}}
$$

From the definition of the function $\gamma_{\alpha}(x, t)$ it is clear that 


$$
\frac{\int_{\left(t_{R E Y}\right.} e(x, t) \gamma_{a}(x, t) d U_{R E V}}{\int_{I_{\alpha}} e(x, t) d U_{a}}=1
$$

therefore

$$
\frac{\overline{\frac{e(x, t)}{}}}{\overline{e(x, t)}}=\frac{U_{\alpha}}{U_{R E V}}=\text { volume fraction of á phase }
$$

In a porous media that is saturated with a single phase fluid, the ratio of the averages of fluid properties is equivalent to the ratio of the interconnected pore spaces to the total rock volume, which is indeed the effective porosity of that rock $\phi$. Hence

$$
\overline{e(x, t)}=\phi \cdot \overline{e(x, t)}^{\alpha}
$$

The averaging in this report will be performed over each phase separately while the GTE in Equation 2.1 was obtained by taking balances over the whole REV. Therefore by assuming that the porous media being modeled is a single phase fully saturated medium we can then apply R.0 to Equation 2.2 and get

$$
\phi \cdot \frac{\overline{\partial e}}{\partial t}=\phi \cdot \overline{-\nabla \cdot\left(e V+J^{E}\right)+\rho \Gamma^{E}}
$$

Note that here the porosity of the medium $\phi$ has already been averaged.

\section{Averaging Rule \#I -- Average of a sum of two properties}

The equation below (R.1) indicates that the average of a sum of two properties is indeed the sum of each property averaged separately. This can be illustrated by considering that, to obtain the average of a large number of samples it is possible to divide that sample into two subgroups and then obtain the average of each subgroup, so 
that the average of the whole large group is the sum of the averages of the smaller subgroups.

$$
\overline{f+g}=\bar{f}+\bar{g}
$$

Now Equation 2.3 becomes

$$
\phi \cdot \frac{\overline{\partial e}}{\partial t}=-\phi \cdot \overline{\nabla \cdot e V}-\phi \cdot \overline{\nabla \cdot J^{E}}+\phi \cdot \overline{\rho \Gamma^{E}}
$$

\section{Average Rule \#2 -- Average of a product of two properties}

The equation below (R.2) indicates that the average of a product of two properties is indeed the product of each property averaged separately in addition to the average of the product of the deviation of each property from its average.

$$
\overline{f g}=\overline{f g}+\overline{f^{\prime} g^{\prime}}
$$

Now Equation 2.4 becomes

$$
\phi \cdot \frac{\overline{\partial e}}{\partial t}=-\phi \cdot \overline{\nabla \cdot e V}-\phi \cdot \overline{\nabla \cdot J^{E}}+\phi \cdot\left(\bar{\rho} \overline{\Gamma^{E}}+\overline{\rho^{\prime} \cdot \Gamma^{E^{\prime}}}\right)
$$

The values $\rho^{\prime}$ and $\Gamma^{E^{\prime}}$ represent the deviations in the value of the extensive property from the averaged values $\bar{\rho}$ and $\overline{\Gamma^{F}}$, respectively. This illustrates the need for an adequate choice of the REV. If this volume can be assumed to be well chosen, then both deviation values must be very small hence the product approaches zero as the choice of REV becomes better. By assuming that the REV was chosen appropriately, Equation 2.5 becomes, 


$$
\phi \cdot \frac{\overline{\partial e}}{\partial t}=-\phi \cdot \overline{\nabla \cdot e V}-\phi \cdot \overline{\nabla \cdot J^{E}}+\phi \cdot \bar{\rho} \overline{\Gamma^{E}}
$$

\section{Average Rule \#3--Time averaged variation of a property}

By invoking Reynolds transport theorem, we are able to obtain the following relationship relating the average of a time derivative of a property $e$ to the temporal derivative of the average of the property $\bar{e}$. In the equation below (R.3) the average of a time derivative of a property $e$ is equivalent to the derivative of the average of the property $\bar{e}$ in addition to any instantaneous transfer of the property $e$ across the interface $\mathrm{S}_{\alpha \beta}$.

$$
\phi \frac{\overline{\partial e}}{\partial t}=\frac{\partial}{\partial t}(\phi \bar{e})+\frac{1}{U_{o}} \int_{S_{\alpha \beta}} e u \cdot n d S
$$

Applying R.3 to Equation 2.6 we get,

$$
\frac{\partial}{\partial t}(\phi \bar{e})+\frac{1}{U_{o}} \int_{S_{\alpha \beta}} e u \cdot n d S=-\phi \overline{\nabla \cdot e V}-\phi \overline{\nabla \cdot J^{E}}+\phi \bar{\rho} \overline{\Gamma^{E}}
$$

\section{Rule \#4--Space averaged variation of a property}

By invoking Gauss theorem, we are able to obtain the following relationship relating the average of a spatial derivative of a property $e$ to the spatial derivative of the average of the property $\bar{e}$. In the equation below (R.4) the average of a spatial derivative of a property $G$, which may be a tensor of any rank, is equivalent to the spatial derivative of the average of the property $\bar{G}$ in addition to any flux of the property e across the interface $S_{\alpha \beta}$.

$$
\phi \overline{\nabla \cdot G}=\nabla \cdot \phi \bar{G}+\frac{1}{U_{o}} \int_{S_{\alpha \beta}}^{G} \cdot n d S
$$


Applying R.4 to Equation 2.7 we get,

$$
\begin{aligned}
\frac{\partial}{\partial t}(\phi \bar{e})+\frac{1}{U_{o}} \int_{S_{\alpha \beta}} e u \cdot n d S=-\nabla \cdot \phi \overline{e V}+\frac{1}{U_{o}} \int_{S_{\alpha \beta}} e V . n d S & \\
& -\nabla \cdot \phi \overline{J^{E}}+\frac{1}{U_{o}} \int_{S_{\alpha \beta}} J^{E} \cdot n d S+\phi \bar{\rho} \overline{\Gamma^{E}}
\end{aligned}
$$

Rearranging Equation 2.8 we get

$$
\begin{aligned}
\frac{\partial}{\partial t}(\phi \bar{e})=-\nabla \cdot \phi \overline{e V}-\nabla \cdot \phi \overline{J^{E}}+\phi \bar{\rho} \overline{\Gamma^{E}} \\
+\frac{1}{U_{o}} \int_{S_{\alpha \beta}} e(V-u) \cdot n d S+\frac{1}{U_{o}} \int_{S_{\alpha \beta}} J^{E} . n d S
\end{aligned}
$$

Now we can describe each of the quantities in equation (2.9):

- $\frac{\partial \phi \bar{e}}{\partial t}:$ Rate of increase of the extensive property $E$ per unit volume of porous media;

- $\nabla . \phi \overline{e V}$ : Net influx of $E$ per unit volume due to advection;

- $\nabla . \phi \overline{J^{E}}$ : Net influx of $E$ per unit volume due to diffusion;

- $\frac{1}{U_{o}} \int_{S_{\alpha \beta}} e(V-u) n d S: \quad$ Amount of $E$ entering the REV due to advection through the interface between the solid and the fluid per unit time;

- $\frac{1}{U_{o}} \int_{S_{a f}} J^{E} . n d S:$ Amount of $E$ entering the REV due to diffusion through the interface between the solid and the fluid per unit time; and

- $\phi \overline{\rho \Gamma^{E}}$ : Amount of E generated in the REV per unit time.

Now we can apply the averaged GTE to each of the three conserved properties mass, momentum, and energy to get the transport equation of fluid in a porous media.

\subsection{Porous Media Formulation}

\section{Fluid Mass Balance}

Using Equation 2.9 to account for the mass of fluid saturating the pore spaces, it is possible to arrive at the fluid mass continuity equation. Here the extensive property 
being modeled is the mass of the fluid, i.e. $\mathrm{E} \rightarrow m^{f}$. As a result, the density of the extensive property $e$ will be the mass density of the fluid $\rho^{f}$. Now Equation 2.9 becomes

$$
\begin{aligned}
\frac{\partial}{\partial t}\left(\phi \bar{\rho}^{f}\right)=- & \nabla \cdot \phi \overline{\rho^{f} V}-\nabla \cdot \phi \overline{J^{m}}+\phi \bar{\rho}^{f} \overline{\Gamma^{m f}} \\
& +\frac{1}{U_{o}} \int_{S_{\alpha \beta}} \rho^{f}(V-u) \cdot n d S+\frac{1}{U_{o}} \int_{S_{\alpha \phi}} J^{m} \cdot n d S
\end{aligned}
$$

Because the rock-fluid interface is assumed to be imperious, there can be no mass transfer across that interface. Therefore, all the surface integrals taken across the rockfluid interface are equal to zero. Therefore, Equation 2.10 becomes

$$
\frac{\partial}{\partial t}\left(\phi \bar{\rho}^{f}\right)=-\nabla \cdot \phi \overline{\rho^{f} V}-\nabla \cdot \phi \cdot \overline{J^{m}}+\phi \bar{\rho}^{f} \overline{\Gamma^{m f}}
$$

Applying averaging rule \#2 to the advective mass transfer term we get

$$
\frac{\partial}{\partial t}\left(\phi \bar{\rho}^{f}\right)=-\nabla \cdot \phi \bar{\rho} f \bar{V}-\nabla \cdot \overline{\phi \rho^{f^{\prime}} V^{\prime}}-\nabla \cdot \overline{\phi J^{m}}+\phi \bar{\rho}^{f} \overline{\Gamma^{m f}}
$$

The new term in Equation 2.12 $\nabla . \phi \overline{\rho^{f^{\prime}} V^{\prime}}$ is the mass dispersion term that represents the spreading of the flow around the granular material of the rock matrix. It is expected that this term will be much less than the advective term proper. This is because the deviation in the fluid density is expected to be small. In addition, since $\bar{V}$ is the averaged velocity it represents flow along the net streamline, hence the deviation away from it, $\overline{V^{\prime}}$, is by definition small relative to it. Therefore, Equation 2.12 becomes

$$
\frac{\partial}{\partial t}\left(\phi \bar{\rho}^{f}\right)=-\nabla \cdot \phi \bar{\rho}^{f} \bar{V}-\nabla \cdot \phi \overline{J^{m}}+\phi \bar{\rho}^{f} \overline{\Gamma^{m f}}
$$


where the molecular mass diffusion term is given by $J^{m}=\rho^{f}\left(V^{m}-V\right)$. This results from the variation between the volume weighted velocity and the mass weighted velocity. However, if we assume that the pore spaces are occupied by a single species fluid that has a uniform density, then it is clear that the molecular diffusion will be zero. Therefore,

$$
\frac{\partial}{\partial t}\left(\phi \bar{\rho}^{f}\right)=-\nabla \cdot \phi \bar{\rho}^{f} \bar{V}+\phi \bar{\rho}^{f} \overline{\Gamma^{m f}}
$$

\section{Rock Mass Balance}

In most practical applications, the mass balance of the rock matrix is not considered as relevant. However in our formulation we are including it at this stage because we are interested in the effect of the acoustic pulse on the rock matrix as a whole, i.e., the dilatation effects of the pulse as well as the question of whether acoustic streaming will lead to transportation of fines within the porous media. Therefore, at this stage, the equation will be considered although it is anticipated that it will complicate the numerical model and may require data that is not readily available. By using the same reasoning as for the fluid mass balance, we obtain

$$
\frac{\partial}{\partial t}\left(\phi \bar{\rho}^{s}\right)=-\nabla \cdot \phi \bar{\rho} s \overline{V^{s}}+\phi \bar{\rho}^{s} \overline{\Gamma^{m s}}
$$

The mass source/sink term in this equation will be neglected; however, it may be a possible term to allow us to model erosion due to cavitations resulting from the acoustic pulse. Therefore the rock continuity equation is

$$
\frac{\partial}{\partial t}\left(\phi \bar{\rho}^{s}\right)=-\nabla \cdot \phi \bar{\rho} s \overline{V^{s}}
$$

In the case of rock dilatation, the velocity of the rock matrix will be given by the substantial derivative of the displacement due to the dilatation. 


\section{Fluid Linear Momentum Balance}

Using Equation 2.9 to account for the momentum of fluid saturating the pore spaces, it is possible to arrive at the fluid momentum equation. Here the extensive property being modeled is the momentum of the fluid along each of the directions parallel to the frame of reference, i.e., $\mathrm{E} \rightarrow M^{f}=m^{f} V^{f}$, and is therefore a vector. As a result the density of the extensive property $e$ will be the momentum density of the fluid $\rho^{f} V^{f}$. Now Equation 2.9 becomes

$$
\begin{aligned}
\frac{\partial}{\partial t}\left(\overline{\rho \rho^{f} V^{f}}\right) & =-\nabla \cdot\left(\overline{\rho^{f} V^{f} V^{f}}\right)-\nabla \phi \overline{J^{M f}}+\phi \bar{\rho}^{f} \overline{\Gamma^{M f}} \\
& -\frac{1}{U_{o}} \int_{S_{\alpha \beta}} \rho^{f} V^{m}\left(V^{m}-u\right) \cdot n d S-\frac{1}{U_{o}} \int_{S_{\alpha \beta}} J^{M f} \cdot n d S
\end{aligned}
$$

The diffusive flux in the momentum equation is given by the term $\vec{J}^{M m}$.

Considering the averaging of fluid velocity with respect to the momentum of the fluid transporting streamlines, it is expected that the magnitude of the momentum averaged velocity will vary from the mass averaged velocity of those streamlines. The variation in those magnitudes of the velocities is equivalent to the stress field acting on the fluid body $\vec{\sigma}^{f}$. However, because the stress field develops to counteract the variation in the velocities, the stress field is proportional to the negative of the variation. Therefore,

$$
J^{M m}=\rho V^{m}\left(V^{M}-V^{m}\right)=-\sigma^{f}
$$

Since it is assumed that the interface $S_{\alpha \beta}$ separating the fluid and solids within the REV is impervious then matter can not pass across it. Hence the dot product of the fluid velocity with the normal to that surface must be zero. Therefore,

$$
(V-u) \cdot n=0 \Rightarrow \frac{1}{U_{o}} \int_{S_{a \beta}} \rho^{f} V^{m}\left(V^{m}-u\right) \cdot n d S=0
$$


Now 2.16 becomes,

$$
\begin{aligned}
\frac{\partial}{\partial t}\left(\phi \overline{\rho^{f} V^{f}}\right)=-\nabla \cdot\left(\phi \overline{\rho^{f} V^{f} V^{f}}\right)+\nabla \phi \overline{\sigma^{f}} & +\phi \bar{\rho}^{f} \overline{\Gamma^{M f}} \\
& +\frac{1}{U_{a}} \int_{S_{\alpha \beta}} \sigma^{f} . n d S
\end{aligned}
$$

The Term $\overline{\Gamma^{M f}}$ represents the body forces acting on the fluid. In our case, the only such force is the gravity force. Hence 2.19 becomes

$$
\begin{aligned}
\frac{\partial}{\partial t}\left(\phi \overline{\rho^{f} V^{f}}\right)=-\nabla \cdot\left(\phi \overline{\rho^{f} V^{f} V^{f}}\right)+\nabla \phi \overline{\sigma^{f}} & +\phi \bar{\rho}^{f} g \nabla z \\
& +\frac{1}{U_{o}} \int_{S_{\alpha \beta}} \sigma^{f} \cdot n d S
\end{aligned}
$$

By applying averaging rule R.3 we get

$$
\begin{aligned}
\frac{\partial}{\partial t}\left(\overline{\phi \rho^{f} V^{f}}\right)+\frac{\partial}{\partial t}\left(\phi \overline{\rho^{f^{\prime}} V^{f}}\right)= & -\nabla \cdot\left(\overline{\phi \rho^{f} V^{f} V^{f}}\right)-\nabla \cdot\left(\phi \overline{\rho^{f^{\prime}} V^{f^{\prime}} V^{f^{\prime}}}\right) \\
& +\nabla \phi \overline{\sigma^{f}}+\phi \bar{\rho}^{f} g \nabla z+\frac{1}{U_{o}} \int_{s_{u \beta}} \sigma^{f} . n d S
\end{aligned}
$$

As before, the deviation of the momentum, i.e., the fluid dispersion, is negligible. Furthermore, the second term on the right hand side of 2.21 is also negligible since the overall change in the velocity vector is expected to be small and the majority of fluid velocity vector deviations will cancel each other out when the balance is taken about the REV Centriod. Therefore 2.21 becomes

$$
\begin{aligned}
\frac{\partial}{\partial t}\left(\overline{\phi \rho^{f} \overline{V^{f}}}\right)=-\nabla \cdot\left(\overline{\rho^{f}} \overline{V^{f} V^{f}}\right)+\nabla \phi \overline{\sigma^{f}} & +\phi \bar{\rho}^{f} g \nabla z \\
& +\frac{1}{U_{o}} \int_{S_{a \beta}} \sigma^{f} . n d S
\end{aligned}
$$


The stress field acting on the fluid flowing in the porous medium is comprised of two components. One results from the pressure within the fluid while the other results from the fluid's viscous intermolecular interaction. Therefore, the stress field can be written as

$$
\sigma^{f}=\tau^{f}-p I
$$

Hence 2.22 becomes

$$
\begin{array}{r}
\frac{\partial}{\partial t}\left(\overline{\phi \rho^{f} V^{f}}\right)=-\nabla \cdot\left(\overline{\rho^{f} V^{f} V^{f}}\right)+\nabla \phi \overline{\tau^{f}}-\nabla \phi \overline{p I}+\phi \bar{\rho}^{f} g \nabla z \\
+\frac{1}{U_{o}} \int_{S_{\alpha \beta}} \tau^{f} . n d S-\frac{1}{U_{o}} \int_{S_{\alpha \beta}} p I . n d S
\end{array}
$$

The last two terms in Equation 2.24 represent the momentum transfer through the fluid-solid interface by shear and normal stress (pressure) respectively. These terms require knowledge of the microscopic shear and normal stresses as well as the configuration of the fluid-solid interface. It is clear that those functions are unknown and therefore we need an alternative averaging formula for these terms. By un-applying the spatial derivative averaging rule (R.4), Equation 2.24 becomes

$$
\frac{\partial}{\partial t}\left(\overline{\phi \rho^{f} V^{f}}\right)=-\nabla \cdot\left(\overline{\phi \rho^{f} V^{f} V^{f}}\right)+\overline{\nabla \phi \tau^{f}}-\overline{\nabla \phi p I}+\phi \bar{\rho}^{f} g \nabla z
$$

\section{Rule \#5-- Space averaged variation of a property satisfying $\nabla^{2} G=0$}

This averaging rule is developed especially to handle surface integrals that require knowledge of the configuration of $S_{\alpha \beta}$ interface as well the spatial distribution of the property $\mathrm{G}$ over that interface. Such surface integrals arise as a result of the averaging of spatial derivatives or a property shown in Rule \#4. Often this knowledge is unavailable; however, knowledge of the derivative of the property over the surface may suffice if that property is known to vary monotonically within the REV. In such cases the following averaging rule can be derived (see Appendix B.1): 


$$
\frac{\overline{\partial \phi G}}{\partial x_{j}}=\frac{\partial \overline{\phi G}}{\partial x_{i}} \frac{1}{U_{o}} \int_{S_{\alpha \alpha}} n_{i} \cdot x_{\mathrm{j}} d S+\frac{1}{U_{o}} \int_{S_{\alpha \beta}} x_{\mathrm{j}} \frac{\partial G}{\partial x_{j}} \cdot n_{i} d S
$$

where the term $x_{j}=$ position vector of point on $S_{\alpha \beta}$ relative to the centroid and $n_{j}=$ unit normal to $S_{\alpha \beta}$ at point $x_{\mathrm{j}}$. The term $\frac{1}{U_{o}} \int_{S_{a \alpha}} n_{j} x_{\mathrm{j}} d S$ is therefore a measure of the momentum of microscopic surfaces on the surface $S_{\alpha \beta}$ about the centriod. This moment describes the tortuosity of the fluid flow as it flows through the REV constricted by the surface $S_{\alpha \beta}$. This tortuosity is defined as

$$
T_{\alpha i j}^{*}=\frac{1}{U_{o}} \int_{S_{u x x}} n_{i} \cdot x_{\mathrm{j}} d S
$$

Applying R.5 to the spatial average of the derivative of pressure in Equation 2.25 we get

$$
\overline{\frac{\partial \phi p}{\partial x_{i}}}=\frac{\partial \phi \bar{p}}{\partial x_{j}} T_{3 i}^{*}+\frac{1}{U_{o}} \int_{S_{j s}} x_{i}^{\prime} \frac{\partial p}{\partial x_{j}} n_{j} d S
$$

Equation 2.26 is written in indicial notation to emphasize that we have converted the averaged spatial derivative along the direction of flow to that which is normal to the confining surface $S_{\alpha \beta}$. Now we require the microscopic pressure gradient that is normal to the surface $S_{\alpha \beta}$. This term can be obtained by analyzing the dominant terms in the $d o t$ product of the unit normal to the surface by the microscopic momentum balance written in indicial notation. This gives

$$
\phi\left(\rho f \frac{\partial V_{j}^{f}}{\partial t}+\rho f_{V_{i}^{f}} \frac{\partial V_{j}^{f}}{\partial x_{i}}-\frac{\partial \tau_{i j}^{f}}{\partial x_{i}}+\frac{\partial p}{\partial x_{j}}+\rho f^{g} \frac{\partial z}{\partial x_{j}}\right) n_{j}=0
$$


Noting that that in the vicinity of this surface the fluid flow is primarily parallel to the surface, we can make the following statement about the dominant terms in the above microscopic equation

$\left|\rho^{f} \frac{\partial V_{j}^{f}}{\partial t}-\frac{\partial \tau_{i j}^{f}}{\partial x_{i}}\right| \cdot n_{j} \ll\left\langle\left|\rho^{f} V_{i}^{f} \frac{\partial V_{j}^{j}}{\partial x_{i}}+\frac{\partial p}{\partial x_{j}}+\rho^{f} g \frac{\partial z}{\partial x_{j}}\right| \cdot n_{j}\right.$

Therefore,

$$
\rho^{f} V_{i}^{f} \frac{\partial V_{j}^{f}}{\partial x_{i}} \cdot n_{j}+\frac{\partial p}{\partial x_{j}} \cdot n_{j}+\rho^{f} g \frac{\partial z}{\partial x_{j}} \cdot n_{j}=0
$$

Now taking the moment of the infinitesimal surface that has $n_{j}$ as the unit normal about the centriod and integrating over the whole surface $S_{\alpha \beta}$, we get,

$$
\begin{aligned}
& \frac{1}{\phi U_{o}} \int_{S_{f s}} x_{i}^{\prime} \frac{\partial p}{\partial x_{j}} n_{j} d S=-\frac{1}{\phi U_{o}} \int_{S_{\beta}} x_{i}^{\prime} \rho^{f} g \frac{\partial z}{\partial x_{j}} n_{j} d S \\
& -\frac{1}{\phi U_{o}} \int_{S_{f}} x_{i}^{\prime} \rho^{f} V_{i}^{f} \frac{\partial V_{j}^{f}}{\partial x_{i}} n_{j} d S
\end{aligned}
$$

The surface integrals on the right hand side represent the gravity force and the drag force at the fluid-solid interface. Simplifying we get

$$
\frac{1}{\phi U_{o}} \int_{S_{f s}} x_{i}^{\prime} \frac{\partial p}{\partial x_{j}} n_{j} d S=-\bar{\rho}^{f} g\left(\delta_{3 i}-T_{3 i}^{*}\right)-\sqrt{\frac{c_{v} \alpha_{m}}{\Delta_{f}^{2}}} \phi \rho^{f}\left|V_{f}-V_{s}\right|\left(V_{f_{i}}-V_{s_{i}}\right)
$$

Now we are in a position to write the macroscopic average for the normal stress on the fluid: 
$\frac{\overline{\partial \phi p}}{\partial x_{i}}=\frac{\partial \phi \bar{p}}{\partial x_{j}} T_{3 i}^{*}-\bar{\rho}^{f} g\left(\delta_{3 i}-T_{3 i}^{*}\right)-\sqrt{\frac{c_{v} \alpha_{m}}{\Delta_{f}^{2}}} \phi \rho^{f}\left|V_{f}-V_{s}\right|\left(V_{f_{i}}-V_{s_{i}}\right)$

A similar treatment for the shear stresses acting on the fluids gives us

$\frac{\overline{\partial \phi \tau_{i j}}}{\partial x_{j}}=\bar{\mu} \phi \frac{\partial^{2} \bar{V}_{i}}{\partial x_{j} \partial x_{j}}+(\bar{\mu}+\bar{\lambda}) \frac{\partial^{2} \bar{V}_{j}}{\partial x_{i} \partial x_{j}}-\bar{\mu} \frac{\phi C_{f} \alpha_{i j}}{\Delta_{f}^{2}} \bar{V}_{r j}$

Finally the fluid linear momentum equation 2.25 becomes

$$
\begin{aligned}
& \frac{\partial}{\partial t}\left(\overline{\phi \rho^{f} V_{i}^{f}}\right)=-\frac{\partial}{\partial x_{i}} \cdot\left(\overline{\phi \rho^{f} V_{i}^{f} V_{j}^{f}}\right)+\bar{\mu}^{f} \phi \frac{\partial^{2} \bar{V}_{i}^{f}}{\partial x_{j} \partial x_{j}}+\left(\bar{\mu}^{f}+\bar{\lambda}^{f}\right) \frac{\partial^{2} \bar{V}_{j}^{f}}{\partial x_{i} \partial x_{j}} \\
& -\bar{\mu}^{f} \frac{\phi C_{f} \alpha_{i j}}{\Delta_{f}^{2}} \bar{V}_{i j}-\phi\left(\frac{\partial \bar{p}}{\partial x_{j}}+\bar{\rho}^{f} g \frac{\partial z}{\partial x_{j}}\right) T^{*}{ }_{j i}-\sqrt{\frac{c_{v} \alpha_{m}}{\Delta_{f}^{2}}} \phi \rho^{f}\left|V^{f}-V^{s}\right|\left(V_{i}^{f}-V_{i}^{s}\right)
\end{aligned}
$$

\section{Rock Linear Momentum Balance}

As discussed above, the nature of the problem we are interested in requires an account of the propagation of the acoustic pulse in the porous medium. This propagation is significantly affected by the presence of the rock matrix and therefore rock momentum equations are included to account for the transfer of both momentum and energy at the fluid-rock interface. Since, the objective of this model is to determine the underlying physical principles by which fluid motion is altered by the presence of cyclic loading, then detailed analysis of all the interactions between the two phases must be considered. The transfer of momentum from the point of view of the solid is mainly to determine the strain undergone by the solid. This strain is responsible for the dilatation of the solid and the resulting effects on the fluid flow. To isolate the strain causing portion of the stress field acting on the solid, we invoke the principle of effective stress in a fully saturated porous medium. The effective stress is given by 


$$
\bar{\sigma}_{s^{\prime}}=(1-\phi)\left(\bar{\sigma}_{s}-\bar{\sigma}_{f}\right)
$$

In order to obtain the solid momentum equation in terms of this effective stress, we add both the fluid and the solid microscopic momentum equation to arrive at a megascopic equation that is in terms of the total stress on the REV:

$$
\begin{aligned}
& \frac{\partial \phi \rho^{f} V^{f}}{\partial t}+\frac{\partial(1-\phi) \rho^{s} V^{s}}{\partial t}=-\nabla \cdot \phi\left(\rho^{f} V^{f} V^{f}\right)-\nabla \cdot(1-\phi)\left(\rho^{s} V^{s} V^{s}\right) \\
& +\nabla \cdot \bar{\sigma}_{t}+\phi \rho^{f} F^{f}+(1-\phi) \rho^{s} F^{s}
\end{aligned}
$$

Then recalling the relationship between the total, effective, and fluid stresses gives us

$$
\bar{\sigma}_{t}=\phi \bar{\sigma}_{f}+(1-\phi) \bar{\sigma}_{s}=\bar{\sigma}_{s}{ }^{\prime}+\bar{\sigma}_{f}
$$

If we substitute Equation 2.37 into 2.36 and remove the fluids momentum equation we have arrived at the solid microscopic momentum equation in terms of strain causing effective stress (Equation 2.38). Recall that this necessary since the solid velocity is only meaningful when it is defined as the substantial derivative of the strain.

$$
\frac{\partial(1-\phi) \rho^{s} V^{s}}{\partial t}=-\nabla \cdot(1-\phi)\left(\rho^{s} V^{s} V^{s}\right)+\nabla \cdot \sigma_{s}{ }^{\prime}+\nabla \cdot(1-\phi) \sigma_{f}+(1-\phi) \rho^{s} F^{s}
$$

Taking the average form of Equation 2.38 and neglecting products of the deviations of averaged properties, we get

$$
\frac{\partial(1-\phi) \overline{\rho^{s} V^{s}}}{\partial t}=-\nabla \cdot(1-\phi)\left(\overline{\rho^{s} V^{s} V^{s}}\right)+\overline{\nabla \cdot \sigma_{s}{ }^{\prime}}+\overline{\nabla \cdot(1-\phi) \sigma_{f}}+(1-\phi) \overline{\rho^{s} F^{s}}
$$


We have already seen the development of the averaged deferential of the fluid stress. In this case, the average is carried out over the solid volume and the arguments are similar to those in the development of the fluid linear momentum equations. Only the final form, in indicial notation, of this average is presented here:

$$
\begin{aligned}
& \frac{\partial(1-\phi) \bar{\rho}^{s}\left(\bar{V}_{i}^{s}\right)}{\partial t}=-\frac{\partial}{\partial x_{j}}\left[(1-\phi)\left(\bar{\rho}^{s} \bar{V}_{i}^{s} \bar{V}_{j}^{s}\right)\right] \frac{\partial \overline{\sigma_{s i j}^{\prime}}}{\partial x_{j}}+\bar{\mu}^{f} \frac{\phi C_{f} \alpha_{i j}}{\Delta_{f}^{2}} \bar{V}_{r j}^{f} \\
& +\phi\left(\frac{\partial \bar{p}}{\partial x_{j}}+\bar{\rho}^{f} g \frac{\partial z}{\partial x_{i}}\right) T^{*}{ }_{j i}-\frac{\partial \bar{p}}{\partial x_{i}}-\left(\phi \bar{\rho}^{f}+(1-\phi) \bar{\rho}^{s}\right) \frac{\partial z}{\partial x_{i}}+\sqrt{\frac{c_{v} \alpha_{m}}{\Delta_{f}^{2}}} \phi \bar{\rho}^{f}\left|V_{r}\right| V_{r_{i}}
\end{aligned}
$$

Now to complete this equation, we require a constitutive relation for the effective stress tensor. In the equations below, the solid is assumed to be an isotropic thermoelastic material that only undergoes small deformations. A detailed description is available in Appendix B, it is sufficient here to include it in general form and the new data that it requires:

$$
\bar{\sigma}_{s i j}^{\prime}=2 \bar{\mu}^{s} \varepsilon_{s k i j}+\bar{\lambda}^{s} \varepsilon_{s k} \delta_{i j}-\bar{\eta}\left(T_{s}-T_{s 0}\right) \delta_{i j}
$$

where $\bar{\mu}^{s}, \bar{\lambda}^{s}$, and $\bar{\eta}$ are the Lamé coefficients of the solid matrix that are to be determined experimentally. The skeleton's strain $\varepsilon_{s k i j}$ is given by

$$
\varepsilon_{s k i j}=\frac{1}{2}\left(\frac{\partial w_{i}}{\partial x_{j}}+\frac{\partial w_{j}}{\partial x_{i}}\right)
$$

where the macroscopic displacement $\vec{w}$ is vector of the solid skeleton and is the volumetric strain: 


$$
\varepsilon_{s k}=\frac{\partial w_{i}}{\partial x_{i}}
$$

\section{Fluid Energy Balance}

By accounting for the total energy in the fluid saturating the pore spaces, it is possible to arrive at the fluid energy continuity equation. Here the extensive property being modeled is the mass of the fluid, i.e., $\mathrm{E} \rightarrow E^{f}$. As a result, the density of the extensive property $e$ will be the mass density of the fluid $\varepsilon^{f}$. Before applying Equation 2.9 , it necessary to simplify the microscopic energy equation so that both the embedded mass continuity and linear momentum equations are removed. This is done to separate the mechanical energy in the system from the thermal energy (see Appendix B.2 for derivations). The new extensive property transported through the system now becomes the internal energy and its density is the specific internal energy $I^{f}$

$$
\rho \frac{D I}{D t}=\tau_{i j} \frac{\partial V_{i}}{\partial x_{j}}-p \frac{\partial V_{i}}{\partial x_{i}}-\frac{\partial J_{i}^{H}}{\partial x_{i}}
$$

Furthermore, this equation is further developed so that the internal energy is replaced by temperature which is a more measurable quantity. This achieved by use of the thermodynamic relationship between internal energy, temperature, entropy, pressure, and volume. The final form of the fluid energy equation that we would like to take an average of becomes

$$
\frac{\partial \rho C_{v} T}{\partial t}=-\frac{\partial}{\partial x_{i}}\left(\rho C_{v} T V_{i}+J_{i}^{H}\right)-\left.T \frac{\partial p}{\partial T}\right|_{v} \frac{\partial V_{i}}{\partial x_{i}}+\tau_{i j} \frac{\partial V_{i}}{\partial x_{j}}
$$

After applying the averaging rules in a similar manner to the above, we can arrive at the final form of the averaged fluid energy equation: 


$$
\begin{aligned}
& \frac{\partial}{\partial t}\left(\phi \bar{\rho}^{f} C^{f} \bar{T}^{f}\right)=-\frac{\partial}{\partial x_{i}}\left(\phi \bar{\rho}^{f} C^{f} \bar{T}^{f}-\phi \lambda_{i j}^{*} \frac{\partial \bar{T}^{f}}{\partial x_{i}}\right)-\alpha *^{H}\left(\bar{T}^{f}-\bar{T}^{s}\right) \\
& -\bar{T}^{f} \frac{\bar{\beta}_{T}}{\beta_{p}} \rho \frac{\partial \phi \bar{V}_{i}^{f}}{\partial x_{i}}+\left(\bar{\mu}^{f}\left(\frac{\partial \bar{V}_{i}}{\partial x_{j}}+\frac{\partial \bar{V}_{j}}{\partial x_{i}}\right)+\bar{\lambda}^{f}\left(\frac{\partial \bar{V}_{z}}{\partial x_{k}}\right) \delta_{i j}\right)\left(\frac{\partial \phi \bar{V}_{i}^{f}}{\partial x_{j}}+\phi \frac{\partial \bar{V}_{i}^{s}}{\partial x_{j}}-\frac{\partial \phi \bar{V}_{i}^{s}}{\partial x_{j}}\right)
\end{aligned}
$$

\section{Rock Energy Balance}

A similar treatment of the rock energy balance allows us to arrive at the following rock energy equation (see Appendix B.2 for derivations). The difference between the two energy equations is that the fluids diffusive transport mechanism is a result of the total stress on the fluid, whereas in the solid it is the strain:

$$
\frac{\partial \rho C_{v} T}{\partial t}=-\frac{\partial}{\partial x_{i}}\left(\rho C^{s} T V_{i}+J_{i}^{H}\right)-T \eta \frac{\partial \varepsilon}{\partial t}
$$

Averaging the above relationship we arrive at the following rock energy equation in terms of the averaged properties of the materials in the medium:

$$
\begin{aligned}
& \frac{\partial}{\partial t}\left((1-\phi) \bar{\rho}^{s} C^{s} \bar{T}^{s}\right)=-\frac{\partial}{\partial x_{i}}\left((1-\phi) \bar{\rho}^{s} C^{s} \bar{T}^{s}-\phi \lambda_{i j}^{* s} \frac{\partial \bar{T}_{i}^{s}}{\partial x_{i}}\right) \\
& +\alpha *{ }^{* H}\left(\bar{T}^{f}-\bar{T}^{s}\right)-\bar{T}^{s} \bar{\eta} \frac{\partial(1-\phi) \bar{\varepsilon}}{\partial t}
\end{aligned}
$$

\subsection{Solution Strategy}

The equations above are developed to enable the isolation of the parameters that are expected to contribute to the transportation of fluid in the porous media under the effect of cyclic pressure loading. In the development of these equations, it is assumed that the pore spaces in the media are fully saturated by a single phase fluid. The strategy for achieving the task objective is to proceed from the simplest case and try to replicate numerically the results obtained from experimental studies.

The numerical solver developed to acquire solutions of the equations requires the equations presented above as well as a number of constitutive relationships achieve 
mathematical closure. The acquisition of these closure relationships has been achieved for the simplified cases and procedures for the more cumbersome cases such as the relationships between tortuosity, shape factors, and the surface areas to the measurable quantities such as porosity and permeability are still under development.

In the following sections some simplification of the equations above are presented along with the results obtained from these simplified models.

\subsection{Model Simplifications}

Acoustic energy transmitted in the near wellbore region interacts with the both fluid and rock formation therein. To understand that interaction, the full set of the complex equations presented earlier must be solved numerically. This task is very complex requiring data that is difficult to collect; furthermore, the results produced are very intricate with interactions embedded into the solution and not obvious to interpret. Therefore, the understanding of the interactions is better achieved by looking at isolated phenomenon and only after the effects of that phenomenon are fully understood and interpreted is it then possible to increase the complexity of the problem by adding more phenomena.

Along this line of thought, it is prudent to ignore the rock-acoustic interaction first. This is because it is not possible to investigate this interaction in the absence of a fluid within the rock. Furthermore, the presence of the fluid will bring us back to the complex problem before any basic understanding has been achieved. As a result, we must look at the fluid-acoustic interactions first and when they are fully understood and interpreted, can we then involve the interactions of the rock formation.

The assumptions needed to isolate the fluid are that the solid is rigid and immobile. However, it may still be a porous media depending on the physical phenomena that is to be isolated. 
To understand the phenomenon of acoustic streaming, the solid-fluid interaction must be kept to a minimum. This is best achieved by studying the flow of a fluid in a steel tubular solid such as a pipe segment. The acoustic pulse is applied to the fluid alone by insuring that the acoustic instrument is not in contact with the pipe wall. The pipe segment is rigid so that solid phase velocity is zero. By applying the pulse for small periods of time, the energy equations of both the solid pipe and the fluid can be neglected because of the limited amount of energy added to the system. Since no appreciable temperature variations in the fluid or pipe will be produced, the system is practically isothermal.

In this numerical experiment, an acoustic pulse shown in Figure 2.2.2 is applied to the fluid in a steel tube 1.0 meter long with a diameter of 1.0 inch. The frequency of the pulse is $15 \mathrm{KHz}$ with amplitude of 0.5 atmospheres. Figure 2.2 .3 shows the history of the velocity of fluid in contact with a point 0.2 meters away from the pipe inlet. In this case, the pulse was applied to fluid at rest within the pipe. The figure shows that the fluid is pushed back and forth over the point with no net fluid displacement after a single pressure pulse passes over the point. This phenomenon is referred to as acoustic streaming. It is believed that this phenomenon is used to acoustically remove unwanted dust particles from microcircuits. The back and forth motion of the fluid exerts a persistent force on the pipe wall as a result of the pipe roughness. This frictional force can be significant when applied on a dust particle that is attached to pipe wall. Furthermore, when applying the same pulse to a fluid that is flowing at steady state within the pipe, we get the velocity history shown in Figure 2.2.4. The figure shows that the same process takes place just as in the no flow case; however, in this case, there is a net translational displacement of the fluid. 


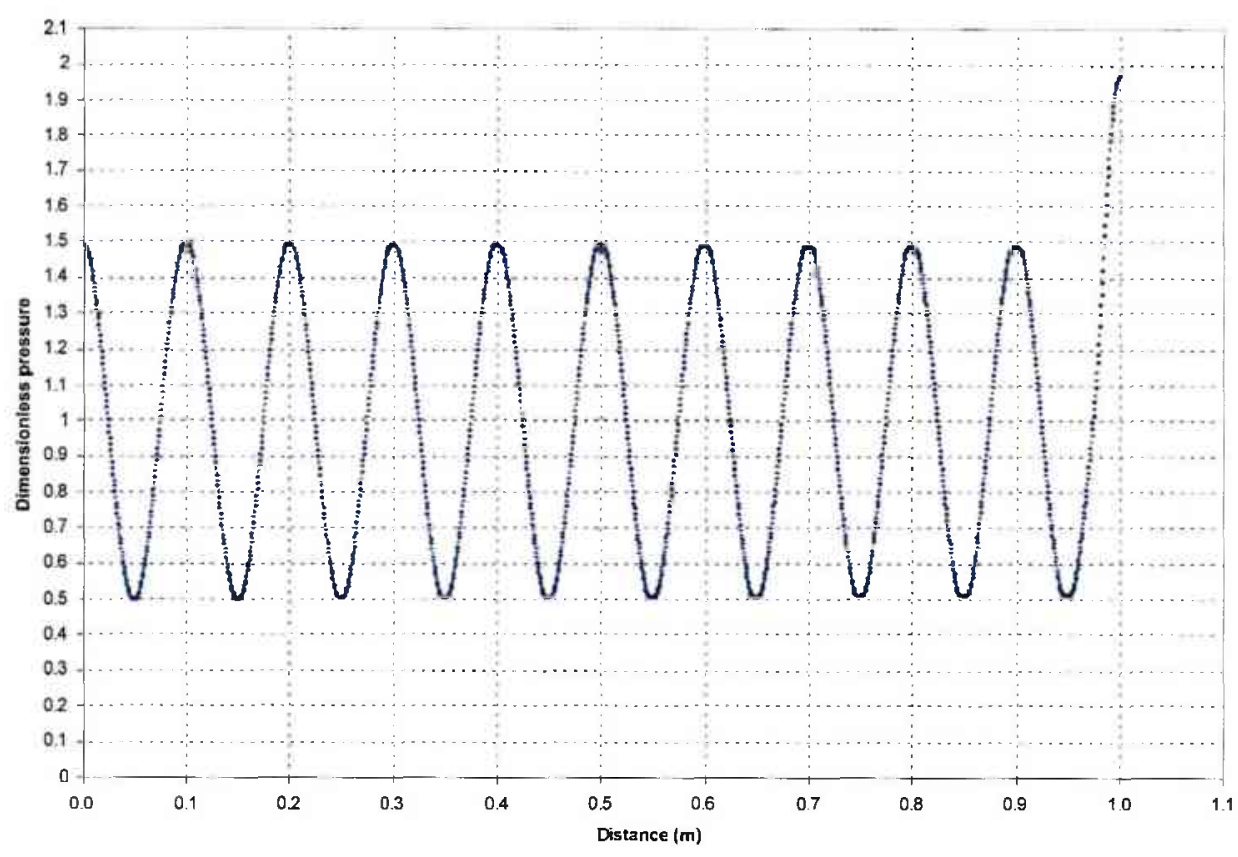

Figure 2.2.2 The pressure profile in the pipe resulting from imposing a sine wave (amplitude $=0.5 \mathrm{~atm}$, frequency $=15.0 \mathrm{kHz}$ )

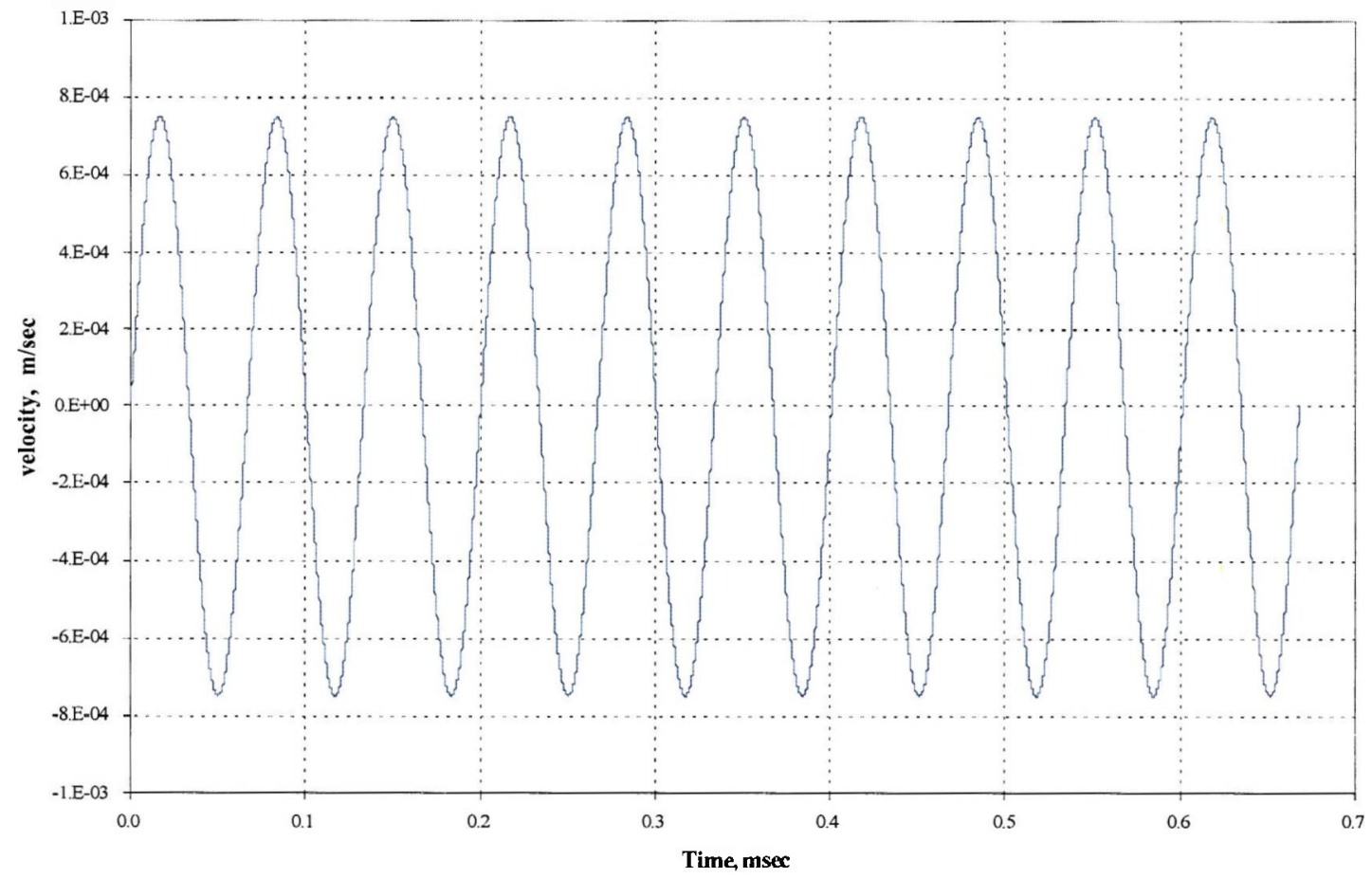

Figure 2.2.3 The velocity history at $0.2 \mathrm{~m}$ resulting from imposing a sine wave on a stationary fluid (amplitude $=0.5 \mathrm{~atm}$, frequency $=15.0 \mathrm{kHz}$ ) 


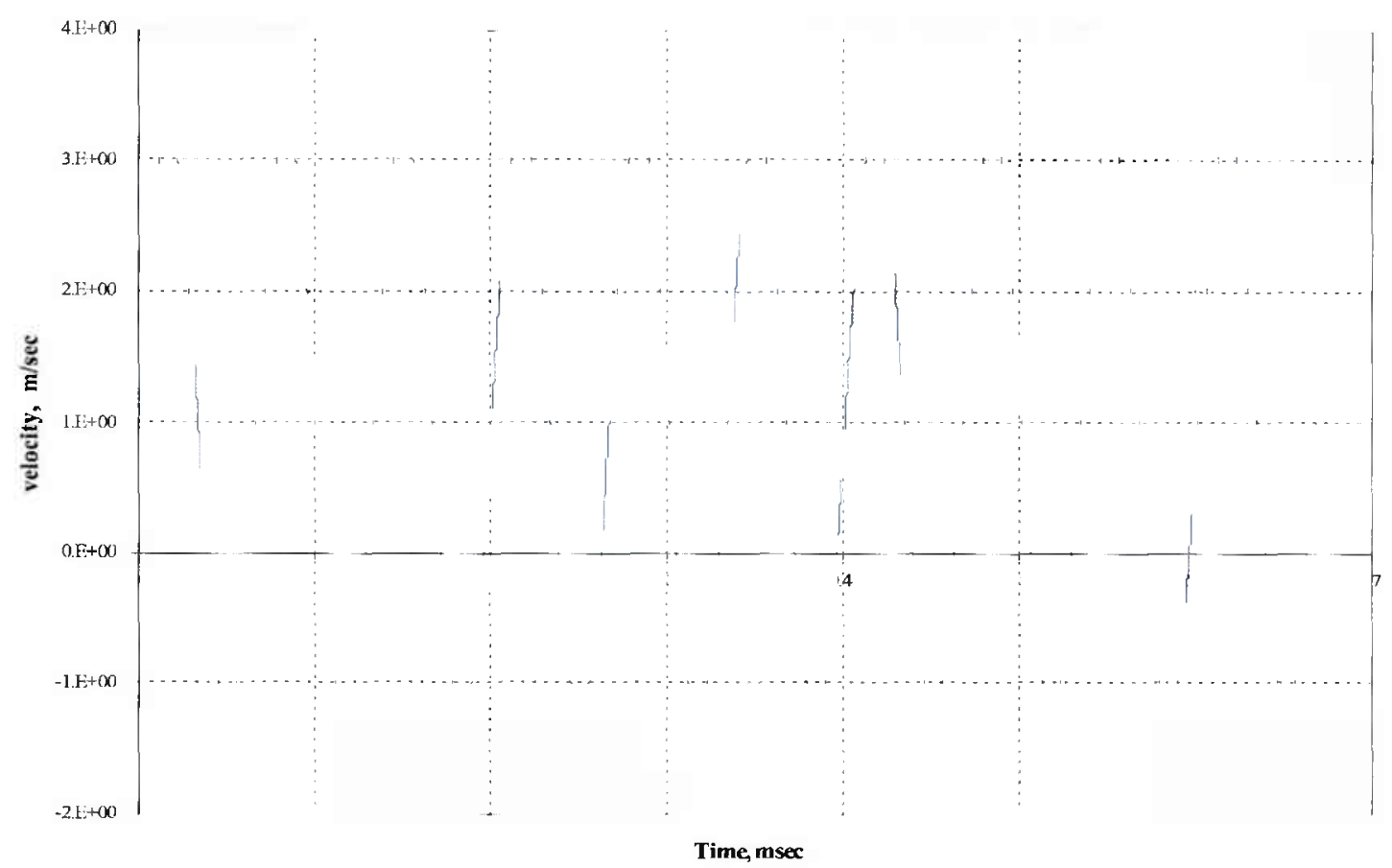

Figure 2.2.4 The velocity history at $0.2 \mathrm{~m}$ resulting from imposing a sine wave on a flowing fluid (amplitude $=0.5 \mathrm{~atm}$, frequency $=15.0 \mathrm{kHz}$ )

\section{Effect of Temperature Variation}

Another experiment was designed to study the effect of converting acoustic energy into an increase in the temperature of the fluid. In this case, we can proceed to add porous medium since we are interested solely in temperature effects. Again, the energy is applied to the fluid alone. This is achieved by applying the pulse to flowing fluid body between the core sample and acoustic transducer. Again the solid phase is immobile, eliminating the need for solid continuity and momentum equations. By limiting the volume of fluid flow, we can insure that the flow within the core would be darcian flow. This means that the fluid momentum equation is replaced by the Darcy's equation for diffusive fluid flow in porous media. Now we are left with the fluid continuity equation and its energy balance. In addition, the solid energy equation is still important because of the large surface area of contact between the fluid and the solid phase. This surface allows for continuous transmission of energy through which it necessitates the need for the solid energy equation. However, as result of the slow diffusive flow and the large contact area between the two phases, the local temperature difference between the two 
phases is negligible. Hence it is possible to combine both energy equations into a single equation as a result of the similar temperature in each of the phases.

Figure 2.2.5 shows the production from the core. The figure shows that the production from a core that is acoustically stimulated is greater than that for one that is not acoustically stimulated. This can be attributed to the reduced viscosity of the fluid as its temperature rises as result of energy infusion. This difference is further illustrated by Figure 2.2.6.

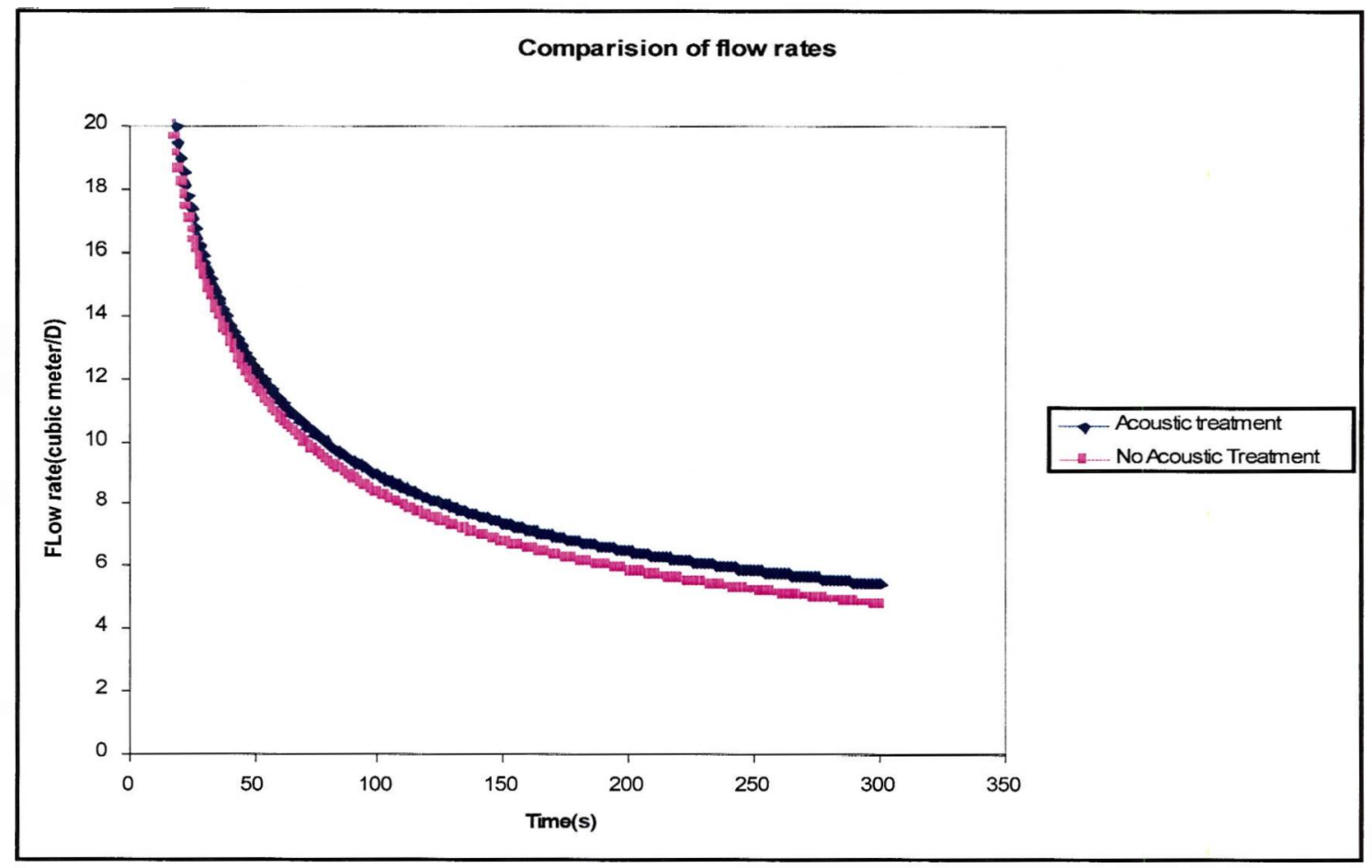

Figure 2.2.5 Comparison showing increase in production as a result of acoustic stimulation 


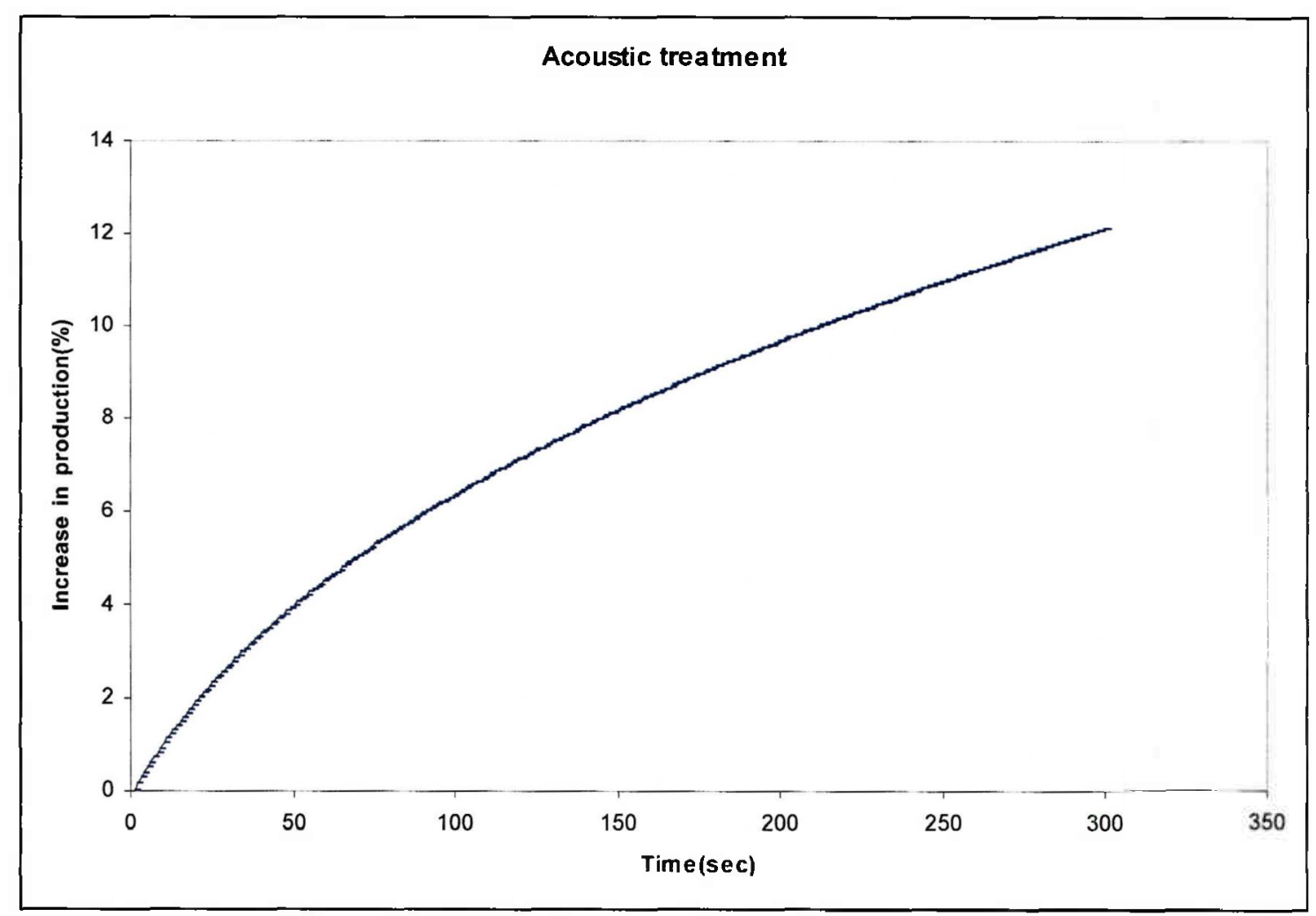

Figure 2.2.6 Increase in production as a result of acoustic stimulation

\subsubsection{Numerical Solver}

A numerical model has been developed to analyze the interaction of acoustic waves with fluid-saturated hydrocarbon reservoir rock. The model was formulated based on the mixture theory approach advanced by Bear and Bachmat [1990]. A numerical model was developed to solve the system of non-linear hyperbolic partial differential equations describing the physical process. This system was solved using an algorithm based on Harten's [1983] Total Variation Diminishing (TVD) method. A Fortran 90 code was written and tested with a shock-tube like problem. The results were in excellent agreement with the analytical solution of the problem. The model was tested with a shock-tube like problem in the porous media and the results were compared with the results of Levy et al. [1996]. Detailed discussion of the numerical model and validation runs are presented in Appendix D.

\subsubsection{Numerical Model to Describe Cavitation Phenomenon}


Cavitation has in the past received much attention in the fields of mechanical engineering and in the areas of hydrodynamics. In previous model efforts, hydrodynamic cavitation was accounted for with the exclusion of acoustic influences. This study developed a source term that may account for both hydrodynamic and acoustic effects with respect to cavitation inception. The resulting source term may then be implemented in a porous media flow model to fully capture the effects of sonication of the near-wellbore region. A model has been developed which is built upon the Euler equations of fluid dynamics. A cavitation source term was incorporated into the gas evolution equation that is activated by a problem-specific Cavitation Inception Marker (CM). It was found that the presence of cavitation events induced by acoustic transducer produces forces that tend to accelerate the fluid toward the acoustic source.

For investigating this phenomenon, a one dimensional fluid flow model which incorporates a cavitation source term was developed. The source term is activated by the cavitation inception marker (CM) developed in this research. CM accounts for acoustic and hydrodynamic factors as criteria for cavitation inception.

The model has been tested and has produced physically meaningful results. The numerical experiments performed during the model validation phase were successful in reproducing a host of expected results. Since laboratory results were unavailable, the numerical validation experiments were used as benchmark for analysis of data. The experiments in which the cavitation source term was activated produced results that are consistent with the physics of the imposed conditions.

It was found that a liquid system undergoing acoustic cavitation experiences a net fluid flow toward the source of the acoustic wave. Furthermore, it can be concluded that cavitation is a self-limiting process. If it is found that cavitation is a useful result of sonication, it is important to note that after sometime, no significant change in the vapor fraction may be achieved. Indeed, this is a useful finding with respect to the selection of an economic duty cycle at which the sonication device may be effective. Further discussion of the findings has been published as a part of this project by Quammie [2004]. 


\subsubsection{Near-Wellbore Thermal Effects of Sonication}

Acoustic stimulation of the oil-bearing strata is accomplished by introducing special vibrations, which are as identical as possible to the natural rock matrix frequency, and/or fluids. These vibrations give rise to several effects in the fluids contained in the strata. They decrease the cohesive and adhesive bounding, as well as a substantial part of the capillary forces, thereby allowing hydrocarbons to flow more easily within the formation. The vibrations cause frictional heat which, in turn, reduces their surface tensions and viscosity. The heating also may cause a partial evaporation of the lighter hydrocarbon fractions. It is obvious that reduced viscosity of the crude oil will favor mobility in porous medium thereby leading to increase in production rates. However increase in production rates may not be sufficient to justify the expenses incurred through consumption of electrical power. Feasibility analyses of enhanced production due to an acoustically-induced heat constitute the main focus of this section. In this investigation qualitative analyses were performed to determine the effect that heat generated through acoustic wave dissipation has on flow enhancement through the near-wellbore region. Laboratory investigations of this effect have been reported in the literature. The primary means of this research work is a numerical model.

The results of this work show that acoustically-induced heat has a short-term effect on the porous media. The vicinity of the wellbore cools down to its original temperature in several days as the produced fluids from porous media carries out the generated heat. Nevertheless, considering short-term effect only and heating mechanism only there is still a strong possibility of successful utilization of acoustic treatment for reservoirs with at least $50 \%$ oil saturation containing high viscosity oils.

The approach adopted in this work was intended to investigate one of the mechanisms involved in acoustic treatment of reservoir formations. From laboratory experiments conducted in the literature it is obvious that the temperature of the core and the fluid inside it rises with acoustic stimulation. The studies show that the temperature rise was 
significant especially in the vicinity of the application point. Temperature rise is attributed to the dissipation of propagating waves in the porous media. The attenuation of the waves in the porous media is high, so the waves cannot propagate far from the source. One can presume that limited penetration of acoustic wave may not be effective in oil production stimulation. Nevertheless, since skin damage is known to occur within several inches of the wellbore, shallow penetration of acoustic waves may produce near wellbore remediation. In this work it was assumed that all acoustic energy dissipates and is converted into heat. This assumption is not entirely valid, since it is clear that it would not be possible to generate heat from acoustic energy with $100 \%$ efficiency. However, in this research study we approach the heating mechanism optimistically in order to determine the limiting advantage of acoustic wave dissipation in porous media.

It was concluded that heating in the immediate vicinity of the wellbore via acoustic treatment is a short term effect. Produced fluids remove the heat generated in the wellbore by the dissipation of propagating acoustic waves. As soon as acoustic treatment stops the cooling of the reservoir takes place via convective flow of the reservoir liquid. Eventually, temperature of the reservoir in the vicinity of the sonic tool cools down to its original value within several days. Thus, it is not possible to explain long term effect of acoustic treatment observed in several field experiments (Beresnev and Johnson, 1994) by the mechanism studied in this work. While our numerical results show an increase in fluid production rate with sonic stimulation, several field observations produced negative results; i.e. the production rates decreases with sonic stimulation. These observations suggest that there might be other factors besides heating mechanism that need to be considered modeling the effects of acoustic treatment.

Considering short term effect, it is possible to claim that heating the immediate vicinity of the reservoir via acoustic treatment may be effective for high viscosity oils and in cases where considerable oil is left in the reservoir. This would suggest that for the matured reservoirs acoustic treatment may not be effective. It was found that for $30 \mathrm{cp}$ oil viscosity there is optimum oil saturation of $50-55 \%$. Oil viscosities below $30 \mathrm{cp}$ are clearly not good candidates for acoustic treatment regardless of oil saturation and 
formation absolute permeability. Oil viscosities of $50 \mathrm{cp}$ and $100 \mathrm{cp}$ can be treated with acoustic energy only above oil saturation of $50 \%$. It can be concluded that best candidates for acoustic treatment are reservoir with oil viscosity of $50 \mathrm{cp}$, oil saturation above $50 \%$ and formation absolute permeability below $1 \mathrm{md}$. Additional details on the results presented in this section have been published as a part of this project by Rejepov [2004].

\subsubsection{Generalized Solver for Hyperbollc PDEs}

Hyperbolic systems of PDEs arise in many practical problems. Therefore, the description and validation of a generalized one-dimensional hyperbolic PDEs solver are presented. This study utilizes the splitting approach to handle the presence of forcing functions, using the Harten's TVD scheme for solving the homogeneous system of PDEs, and the fifth-order Cash \& Karp Embedded Runge-Kutta method for solving the source problem, which is a system of ODEs. The outcome is an efficient, maintainable, and useful program that minimizes the data required, and can be adaptable to any problem without altering the original code. The solver was validated by solving the most general case of a system of hyperbolic PDEs and its simplifications, among which are some classical Benchmark problems like the Linear Advection, Inviscid Burgers equation, Euler equations and a non-homogeneous scalar model equation; moreover, the numerical solutions are compared against their analytical counterparts. For the specific case of a non-homogeneous system, the Detonation Analogue proposed by Fickett has been used in the past, but we found it to be improper to validate our solver. Hence, due to the lack of problems of this type with exact solution, we have designed our own non-homogeneous system with its analytical solution, which make it useable as a Benchmark problem for this solver and future studies. Complete discussion of this generalized solver is presented in appendix E. The information presented in appendix $E$ was submitted for publication by Leon \& Adewumi on May 2004. 


\subsection{Task 3. Development of a New Generation of Acoustic Transducers}

The activities of this task include enhancing the slim-tube test methodology, refining the test hardware, and documenting the experimental design. Test matrices were developed to organize the test configurations and the range of parameters to be measured. The results were used to establish the types of sensors and signal conditioning equipment needed for the slim-tube apparatus. Parameters to be investigated include frequency, acoustic intensity, duty cycle, static pressure, and temperature. These tests and their results will be used to determine the operational parameters yielding the optimum performance of a field-ready ensonification system. In this case, an optimal system would promote the highest permeability for a given substrate. Three piezoelectric transducers, 2 rated at $20 \mathrm{kHz}$ and 1 rated at $40 \mathrm{kHz}$, were used to carry out initial investigations on the effects of sound-structure interactions.

Transducers, projectors, and rarely hydrophones, are the primary components of acoustic-enhanced fluid-flow and near-wellbore damage experiments. Projectors are often obtained from vendors for the generation of pressure waves in liquids. Users are often limited by the manufacturers technical specifications and are therefore forced to operate within available settings. Consequently, systematic investigations of flowenhanced parameters are poorly defined and irreproducible. As an example, Gollapudi, et al., (1994) used an ultrasonic method for the removal of asphaltene deposits during petroleum production. They did not specify the brand or model of the commercial ultrasonic transducer (distruptor horn) used. In addition, the output values were stated in the form of the output control settings, but not in terms of power (watts) or acoustic intensity. The reproducibility of such an experiment may be difficult, if not impossible, due to insufficient information. The need to understand the governing mechanisms of acoustic energy-fluid-solid interactions in porous media requires versatile transducers that can be operated within various parameters. Transducers were supplied by ARL to carry out basic experiments at room temperature and various static pressures in order to shed some light on the acoustic energy-fluid interactions in porous media. 


\subsubsection{Transducers and Hydrophone Callbration}

Three piezoelectric projectors, two $20 \mathrm{kHz}$ and one $40 \mathrm{kHz}$ were supplied for laboratory-scale experiments. They are "low Q" transducers, which allows them to be used for a short pulse of ultrasound consisting of a broad range bandwith of frequencies. One projector has a square radiating head with a total area of $12.25 \mathrm{~cm}^{2}$. The other transducer's radiating face was ground to a circular form with a reduced area of $9.62 \mathrm{~cm}^{2}$, for easy coupling into the test apparatus. The $40 \mathrm{kHz}$ circular projector has a radiating face of total area $7.07 \mathrm{~cm}^{2}$. Two Polyvinylidene Fluoride (PVDF) hydrophones embedded in polyrethane, one circular $\left(5.07 \mathrm{~cm}^{2}\right)$ and the other rectangular $\left(6.45 .0 \mathrm{~cm}^{2}\right)$ were supplied by ARL. The circular hydrophone was calibrated in the ARL fresh-water pool, assuming the speed of sound to be $1,480 \mathrm{~m} / \mathrm{second}$ and density $1,000 \mathrm{~kg} / \mathrm{m}^{3}$. The hydrophone receive response is calculated from measurements of the source transducer and hydrophone voltage waveforms. Free field voltage sensitivity (FFVS) or the receive sensitivity is typically defined as $\mathrm{dB}$ relative to 1 volt per micropascal input (Figure 2.3.1). The figure shows measurements taken with and without lead urethane acoustic backing as a function of frequency.

\subsubsection{DetermInation of Resonance Frequency of a Branson Horn}

The resonance frequency of a Branson transducer was determined at ARL in order to be able to operate it in non-cavitation and cavitation regimes, using other available power supply and amplifier. The transducer's accompanied factory power converter and power supply (model 250) are designed to energize the transducer to the cavitation level regardless of the duty cycle, mode of operation (pulse/continuous wave) and output control setting. This particular transducer is considered to be a "high Q" transducer. It responds to a short voltage pulse with a relatively long lasting vibration decay time, emitting ultrasound with a narrow bandwidth. The resonance frequency was determined to be $24 \mathrm{kHz}$. These transducers were used to carry preliminary investigation to elucidate sound structure interactions, especially crude oil and water. 


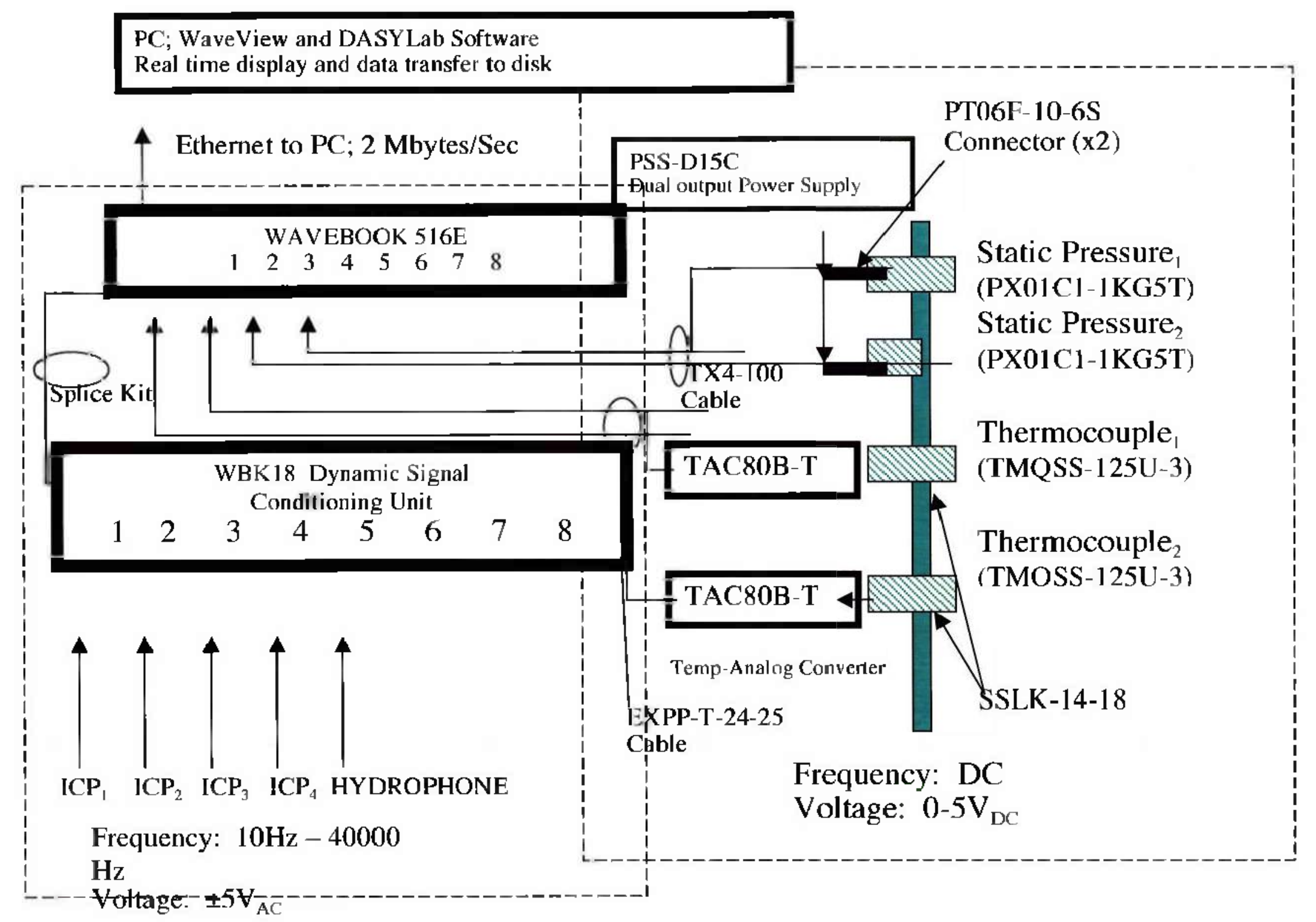

Figure 2.3.I Equipment settings for data acquisition and analysis. 


\subsubsection{Determination of the Equipment Requirement for Fabrication of the Slim-Tube Apparatus}

The key to the success of laboratory investigations of acoustically enhanced fluid flow in porous media and wellbore improvement depend on the ability to control and regulate applying acoustic energy. The purpose of the slim-tube experiments were to determine the optimal system operating parameters for a field unit and quantify its performance for known fluid bearing media. The high level ensonification system objectives are to have a sonic projector that can fit down the well hole, does not interfere with the well operation, is highly efficient, and is reliable in the well environment. In order to meet those top-level goals, this program must determine the "best" set of operating parameters for the system. As such, the laboratory portion of the experiments were to be used to explore the effect that sonic frequency, average acoustic intensity, cavitation, temperature, and static pressure have on flow through various media.

\subsubsection{Dynamic Pressure Transducer}

In addition to measuring static pressure in the slim tube, dynamic pressure will be measured and used to estimate the acoustic intensity in the media. The acoustic intensity is defined as the power per unit area delivered to the oil-bearing medium. This data is important in order to determine the design of a projector for use in the wellbore and to quantify the amount of energy required for flow enhancement. The efficiency of a device is the ratio of the output power to the required input power. Ultimately, the end device will have to produce certain intensity in the medium efficiently. Hence the need to determine flow as a function of intensity, or power delivered to the media. Theoretical estimations of the magnitude of sound pressure to be sensed by both the dynamic (acoustic) and static pressure transducers were carried out as a function of material property. For example, Figure 2.3.2 shows dynamic pressure (psi-rms) and dynamic signal level (V-peak) as a function of acoustic intensity (Watts $/ \mathrm{cm}^{2}$ ) response to dynamic pressure in sand or water medium. The pcb112A22 Piezotronics ${ }^{\text {dignamic }}$ pressure transducer was chosen because it shows the highest sensitivity over that range of intensities. Also, its integral signal conditioning is compatible with the WaveBook. 


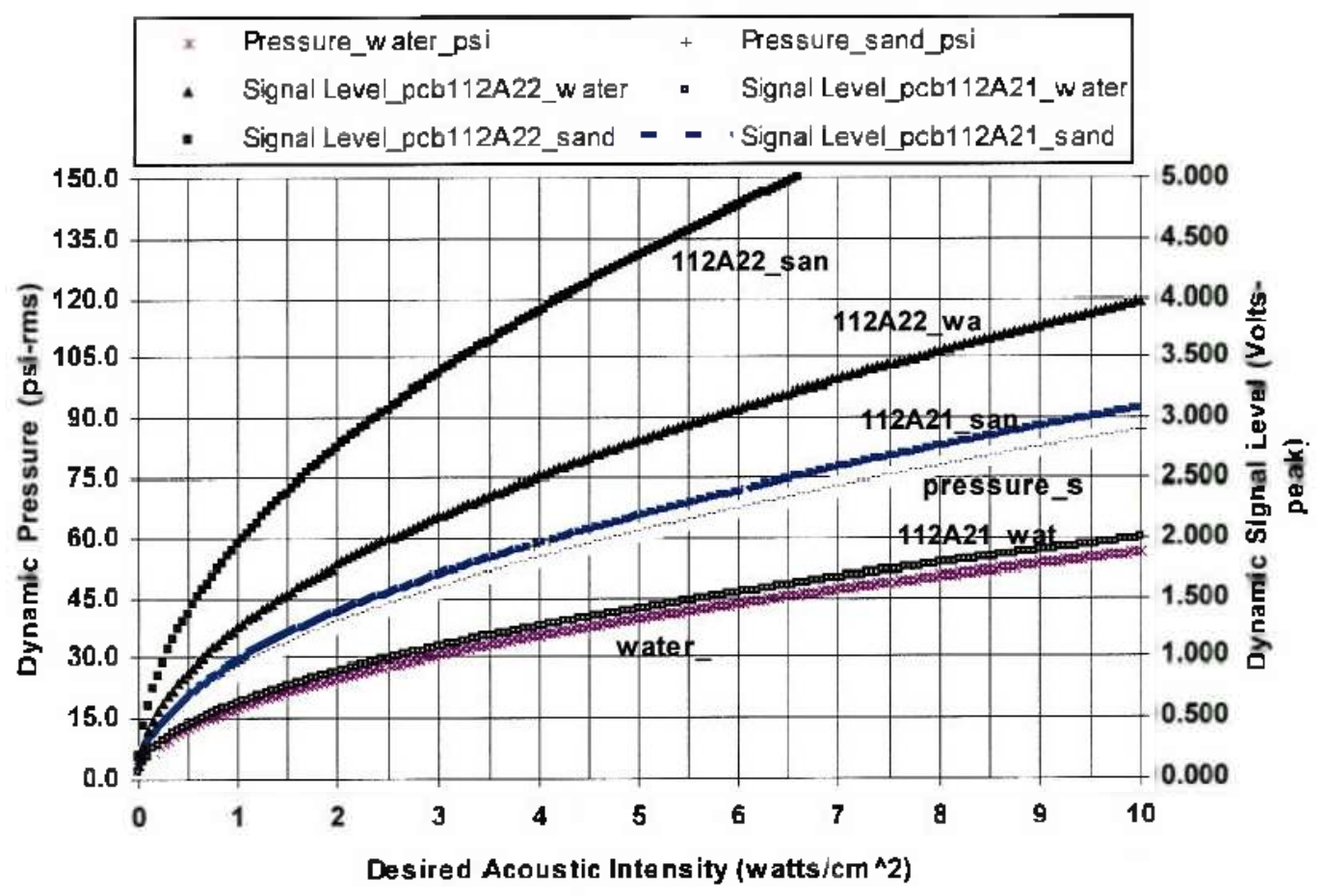

Figure 2.3.2 Theoretical estimation of dynamic pressure levels in the slim-tube apparatus

\subsubsection{Tests and Measurements}

The main variable that will be monitored in future tests as functions of other parameters, is the change in the flow rates (permeability) of fluid (crude oil, brine, water or Soltrol 170) with respect to investigated parameters. The flow rates will be calculated for the following test parameter variants:

- Intensity and frequency, for a fixed duty cycle;

- Duty cycle versus frequency, for a fixed intensity;

- Temperature and intensity, for a fixed frequency; and

- Vary static pressure for the above combinations.

The results will be displayed in 3-D plots in order to determine the maximum, or optimal, flow as a function of those variables. The number of runs per unit time for a combination variable will determine the rate and volume of data generated. For example, experiments requiring 5 different fixed excitation frequencies, 3 varying duty cycles and 
3 different intensities will result in $\mathbf{4 5}$ data sets: 5 frequencies $x 3$ duty cycle $\mathbf{x} 3$ intensities.

Figure 2.3.1 shows the data acquisition configuration, after considering such factors as time, volume of data, sampling interval and frequency, compatibility and so on. The setup consists of WaveBook/516E, WKB18 and DASYLab software. These items are supplied by IOTECH, OH. The WaveBook/516E is a 1-MHz, 16-bit Ethernet-based portable data acquisition system that will be connected to a computer. WBK18 is an 8Channel signal-conditioning module that will receive input from dynamic pressure sensors. Dynamic pressure transducers model 112A22 were obtained from PCB Piezotronics. Thermocouple (TMQSS-125U-3), static pressure transducers (PX01C11 KG5T), analog converters (TAC-80B-T), cables and other connectors were purchased from Omega Engineering. The equipment were configured and calibrated after setup. 


\subsection{Task 4. Field Testing of the Sonication Technology}

The overall plan for Task 4 was to perform field trials of the sonic transducer design. These trials were to take place in production and/or injection wells located in Pennsylvania, New York, and West Virginia. The field trials were expected to require three months of preparation time and one year of testing time.

\subsubsection{Phase 1 Activities}

Work performed during the first phase included the start of several tasks leading towards the actual field testing of the sonication equipment. Examples of these tasks are literature searches, meetings with producers, information and sample gathering, and drilling four new wells for project use.

A literature search and review of four targeted production formations was initiated. Production from the Glade, Clarendon, and Gordon formations has occurred for over 100 years. Due to this length of time, the most recent information found on these formations was from the mid 1960s. The bulk of the Bass Islands production is more recent and the literature dates to the early 1990s. Copies of this information can be found in Appendix C.

Other work included meeting with four independent oil producers to organize plans for the project. Plans varied for each operator, depending on the quantity and types of wells to be used in the project. This work is described in the subtasks listed below.

\subsubsection{Evaluation in Partially Depleted Sandstone}

The operators involved in this subtask were I.L. Geer and Sons (ILG) and Metzgar Oil Company (MOC). Located near Warren, Pennsylvania, both operators produce oil, water, and gas from either the Glade formation or a combination of Glade/Clarendon formations. ILG drilled four new wells for the project while MOC contributed an older production well.

ILG drilled and completed four wells during fall 2002 in the Glade Field. This field is located near Warren, Pennsylvania and has been producing for over 100 years. The four new wells penetrate the Glade, Clarendon and Balltown formations. Similar to most wells in this region, the completion method is openhole style. Figure 2.4.1 shows a 


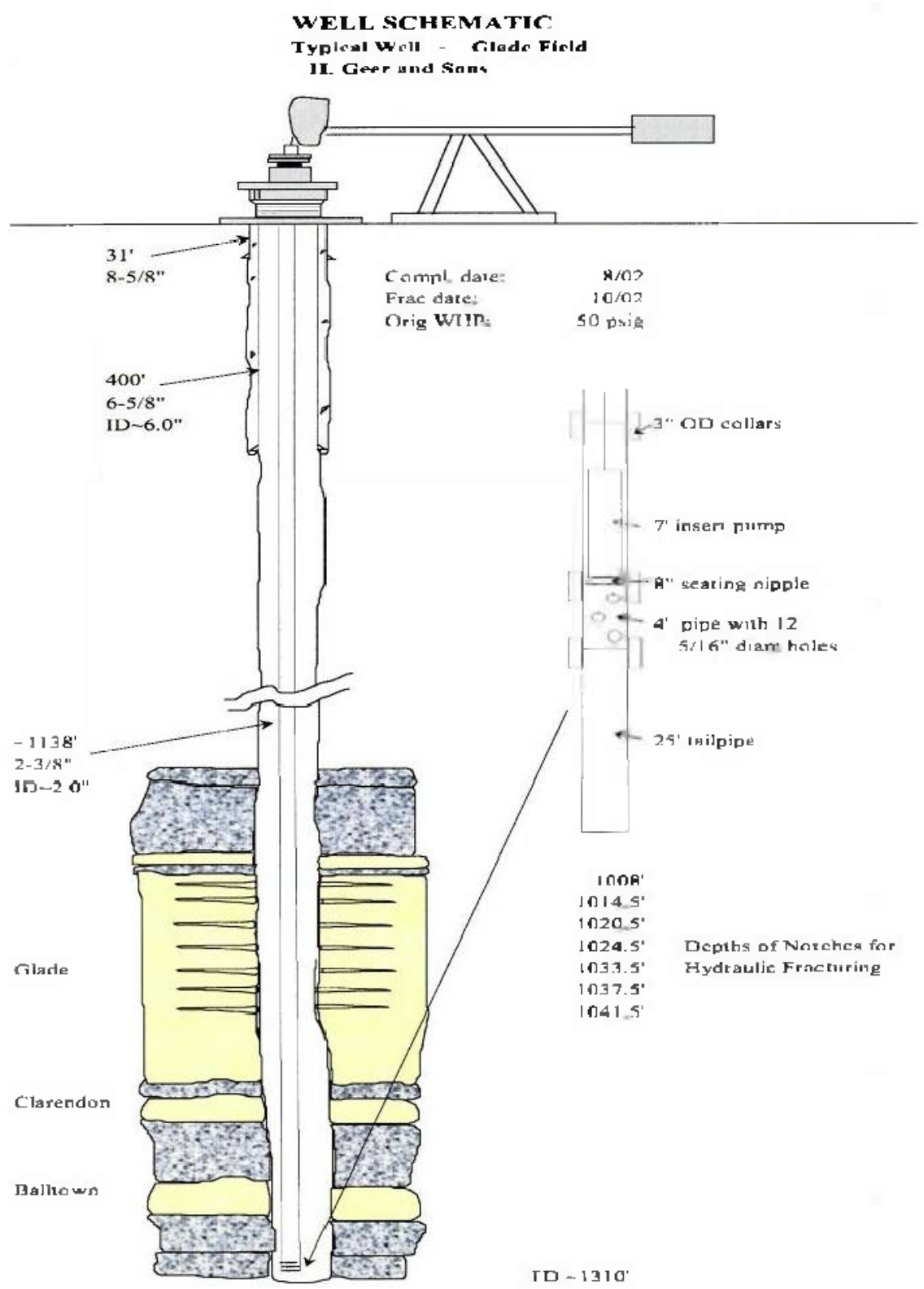

Figure 2.4.1 Schematic diagram of a typical completion for the ILG wells

typical completion for the ILG wells. Next, the wells were logged using Schlumberger's Air-Quad logging suite of gamma-ray, litho-density, compensated neutron, array induction, temperature and caliper logs. This suite was used because it provided excellent resolution, calculated porosities, and calculated water saturations. Figure 2.4 .2 shows a section of log section of the Glade sandstone. After air-notching the sandface at multiple depths, the wells were hydraulically fractured using a mixture of water, sand and 
additives. Following cleanup, the wells were piped into service with a beam pumping unit for lifting liquids and a gas meter to measure natural gas production. A separate 100 barrel tank was set for each well. Figure 2.4.3 shows the surface equipment arrangement for a typical well.

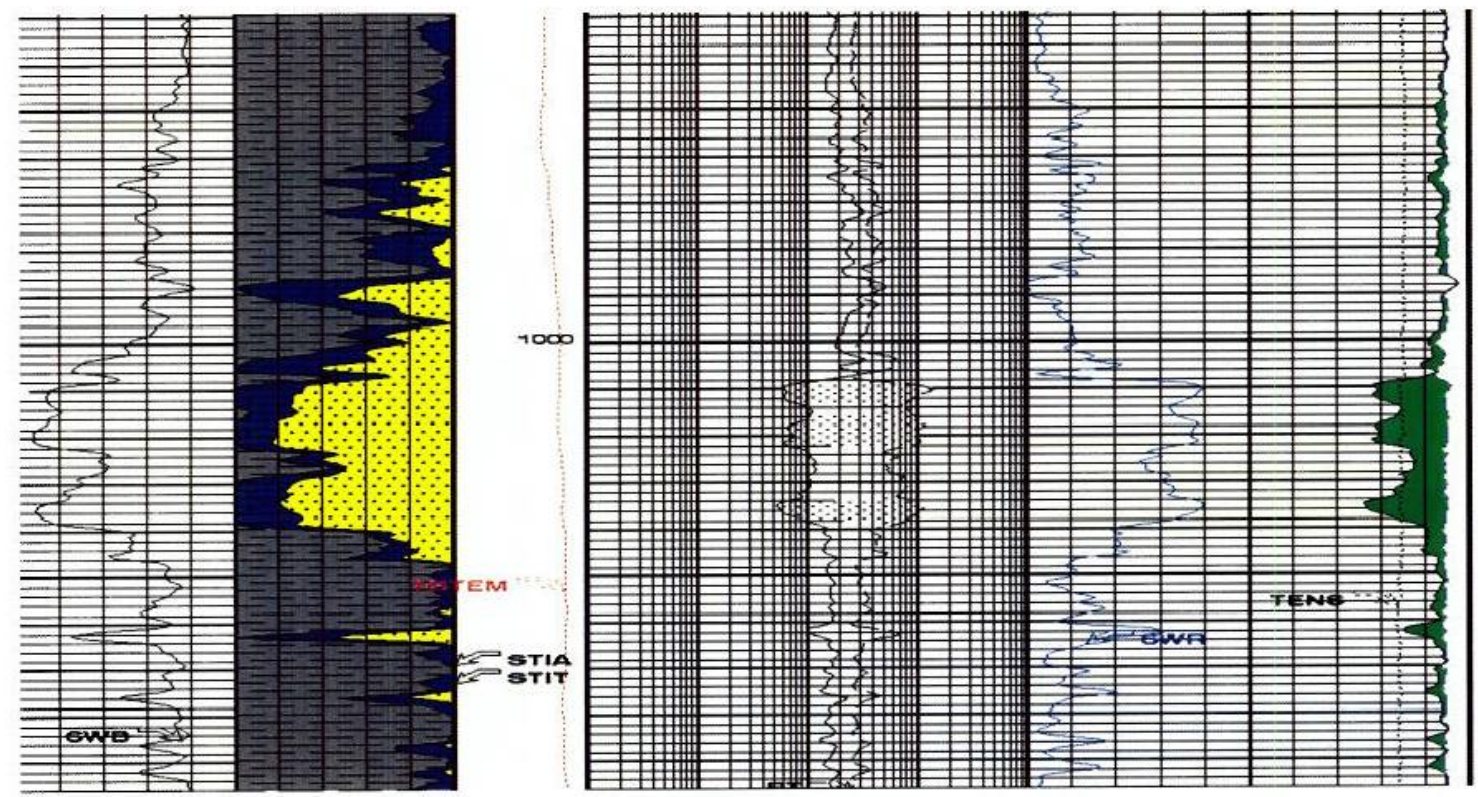

Figure 2.4.2. Section of the Glade Sandstone log section

Praduction from the four wells has changed from mostly fracturing fluids to a mixture of oil, water, and natural gas. Production amounts were recorded to establish a baseline. This baseline was to be used to determine the success of the sonication equipment. Figure 2.4 .4 shows oil, water and natural gas production from one of the project wells. The natural gas measurement for these four wells was performed using meters that were donated to the project by Utilities \& Industries, Inc, of Reynoldsville, Pennsylvania.

During November 2003, IOG drilled a new well located west of project well 65. Drilling was started using a conventional hammer-drill bit. The drilling equipment was switched to a four-inch diamond coring bit near the estimated top of the Glade formation. About eighty feet of core were taken at depths from 870 to 950 feet. Several project team 


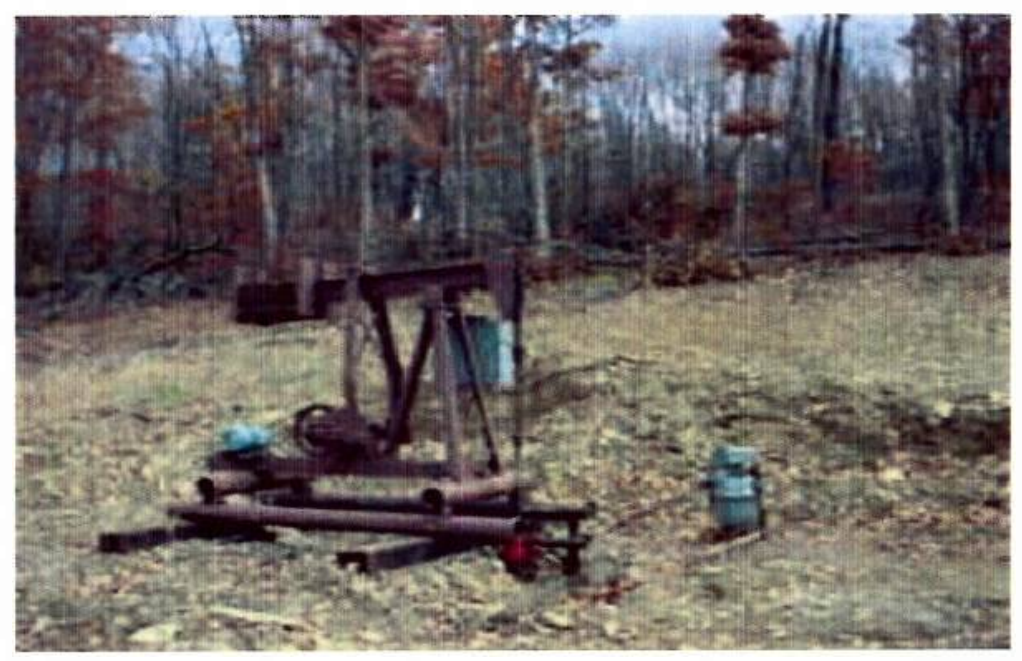

Figure 2.4.3 Equipment arrangement for a typical well

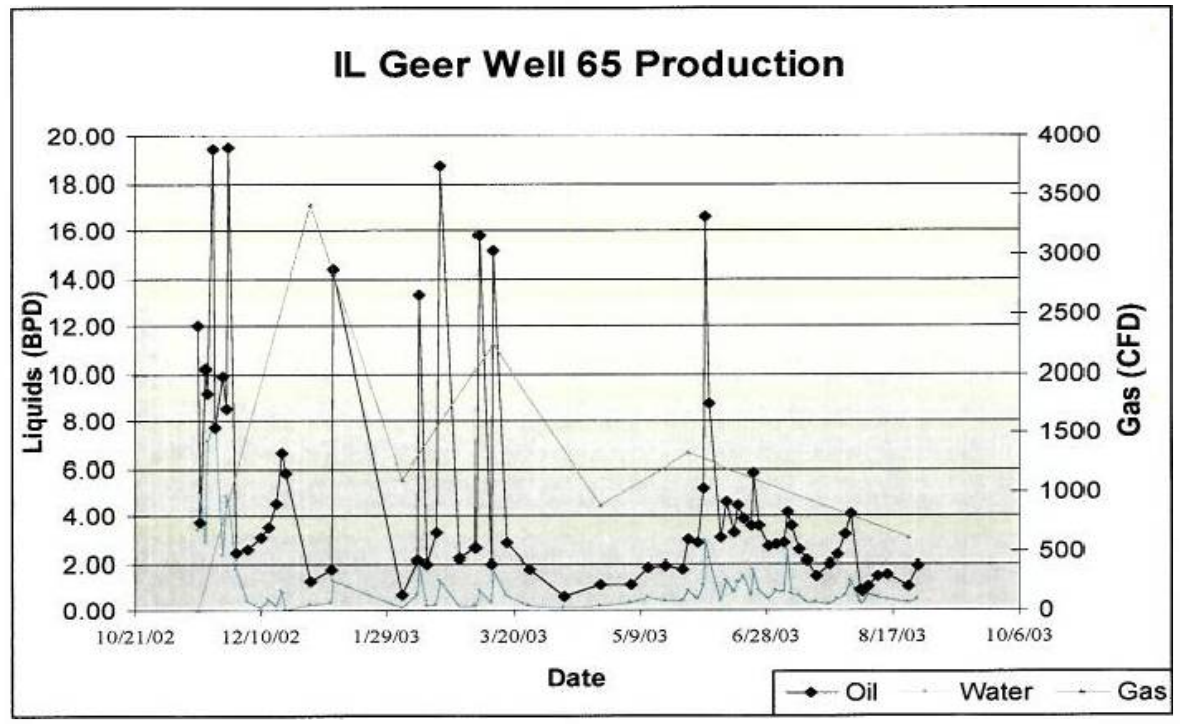

Figure 2.4.4 Production from a typical well

members went to the location to pack the cores into boxes and transport them to Penn State for analysis. Core analysis plans included drilling plugs from the core at various intervals and testing the plugs for saturation, porosity, and permeability.

The MOC well penetrates the Glade and Clarendon formations. This well has produced for many years. Pumped once per week, this well produces around three- 
quarters of a barrel of oil per week along with some water and natural gas. Original well and production records were not located.

We received oil, production water, paraffin and fracturing sand samples from ILG for laboratory testing. Our plans for these wells were to monitor the oil, water and natural gas production to establish a baseline. The plans were to then test the sonication equipment in these wells for approximately one year; however, this activity will not be performed since the final two phases of the project will not be performed.

\subsubsection{Evaluation in a Shot Hole}

Our plan for evaluating the sonication equipment in a shot hole was to request the use of one well from ILG near Warren, Pennsylvania. A well was chosen and most of the surface equipment had been installed. Next, we were to install measurement equipment to monitor the oil, water and natural gas production. Production would have been measured for at least three months to establish a baseline. Finally, we were test the equipment in their well for about one year. Again, this activity will not be performed.

\subsubsection{Evaluation in a Hydro-Fractured Wellbore}

Our plan for evaluating the sonication equipment in a hydro-fractured wellbore was to request the use of one or more wells from East Resources (ER). ER is located close to Pittsburgh, Pennsylvania and has several Gordon formation waterflooding operations in Pennsylvania and West Virginia. The Gordon formation is a sandstone bed that has been produced for over 100 years.

The candidate wells were to be injection wells that ER uses to inject treated water for their waterflooding operation. After choosing the candidates, we were to prepare measurement equipment to monitor the water injection rates. Injection was to be measured for at least three months to establish a baseline. Finally, we were to test the equipment in their wells for around one year. This activity will not be performed.

\subsubsection{Evaluation in a Fractured Carbonate}

Lenape Resources (LR) was the operator involved in this subtask of the project. Located in Botavia, New York. LR produces oil, water and natural gas from the Bass 
Islands. Bass Islands is a term used by drillers in the area when referring to the AkronBertie group of New York. Presently, LR has one candidate well with electricity access. This well, located near Jamestown, New York, produces a substantial amount of water and has problems with paraffin.

We met with LR several times and discussed well plans. A Bass Islands oil sample was received from LR for laboratory testing. Our plans for this task were to gather past and future production data from this well and establish a baseline. Finally, we were to test the equipment in their well for approximately one year. This activity will not be performed.

\subsubsection{Oil Wells Under Water Drive}

The operator involved in this subtask of the project was East Resources (ER). ER is located close to Pittsburgh. Pennsylvania and has several Gordon formation waterflooding operations in Pennsylvania and West Virginia.

Work completed in this activity included characterizing the Gordon sandstone at the Wileyville field. This field and its wells were to be used for the testing and evaluation of the Sonication device. The Wileyville field presented an interesting challenge in the context of the application of acoustics for removal of damage in the wellbore. The Wileyville field is currently undergoing waterflooding. Waterflooding was initiated in 1997 and prior to that gas injection was tried during the middle of the last century. The problem experienced by the field operator amounts to the maintenance of the water injection rate. To date, the operator periodically hydrofractures the injection wells to maintain well injectivity. The objective of testing the sonication device in these injection wells was to evaluate the mitigation of skin damage using the acoustic device while maintaining injectivity.

The Wileyville field is located in Wetzel County, West Virginia. It was discovered in the late 189's. In terms of size, it occupies approximately 308 acres. Geologically, it was thought to be an isolated Gordon sandstone reservoir, but it is in fact, connected to a similar Gordon sandstone field and thus forms a reservoir complex. The top of the Gordon sandstone in this field is found between 1,800 and 1,950 feet below sea level. The deepest part of the field is located in the northern part of the reservoir. The 
potential gradient is about 180 feet per foot of depth in the north-south direction where the length is 27,000 feet. In the east-west direction the difference between the deepest and the shallowest portion of the reservoir amounts to 20 feet. The linear distance between these points is approximately 5,500 feet, with a resulting gradient of 275 feet of linear distance per each foot of depth in the east-west direction. The gravitational gradient appears to be higher in the north-south direction and impacts evaluation of the reservoir, since the preferred flow path is developed in this direction.

The thickness, like most of the properties in this field, has a very inegular distribution. Towards the north and the east boundaries the sand tends to thin out, with values averaging 4 to 6 feet. Meanwhile the pay section tends to become thicker toward the western portion of the reservoir. This is especially true toward the center-west portion of the reservoir where average thickness is approximately $11 \mathrm{feet}$, with some areas where thicknesses average 15 feet. The transition in formation thickness between these regions occurs in the middle of the field where the principal flow path is located and as previously noted trends in the north-south direction.

In the Wileyville field, porosity and permeability characteristics control the location of oil accumulation and the relative productivity of various areas and individual wells. Analysis of these properties indicates that the northwestern region of the field is separated from the southern region by a strip that is approximately 500 feet wide and extends across the entire field from east to west. The porosity of this area ranges from 2 to $6 \%$. This is in contrast to a total reservoir average of $20 \%$. As expected, the porosity tends to decrease towards the reservoir boundaries and the highest values, around $36 \%$, are located in the central region (see Figure 2.4.5).

The northwestern area represents a third of the field's areal extent and has permeability values in the center as high as $175 \mathrm{md}$. The permeability of the central and southern edges of the reservoir can range from 10 to $20 \mathrm{md}$. The strip crossing the field has values of permeability ranging between 5 and $10 \mathrm{md}$ and is below the field average of $45 \mathrm{md}$. As previously noted, this strip acts as a boundary in terms of limiting fluid movement between the north and the south (see Figure 2.4.6). 


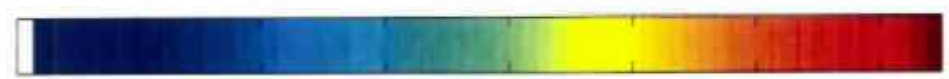

Figure 2.4.5 Wileyville field, isoporosity map

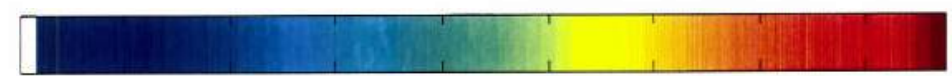

Figure 2.4.6 Wileyville field, isoperm map

Regarding the saturation distributions, the average saturation for both water and gas was assumed to be $25 \%$. These assumptions were provided by the field operator and 
were based on the experience of the field operator. No records or tests were available to support this assumption. It should be noted however that the operator has extensive experience in the operation of wells in the Appalachian Basin. The water saturation was assumed to be uniformly distributed over the field. The exceptions to this are areas to the east and to the west in the northern part of the line-drive injection where water saturations were determined through history matching to be in the range of 10 to $15 \%$. As previously stated, gas saturation takes into consideration the gas injection project in the southern portion of the field have values of up to $40 \%$. Oil saturation averages $50 \%$ across the entire field. The exceptions are the oil saturations found in the northern and southern portions of the field. The oil saturations in these areas are 65 and $70 \%$, respectively. Using these oil saturation values, it was estimated that $2.4 \mathrm{Mbbl}$ of oil are recoverable through waterflooding.

The Wileyville field consists of 22 active production wells. Thirteen of these wells are located to the west of the injection wells and 9 are located to the east. The locations of these wells are shown on Figure 2.4.7.

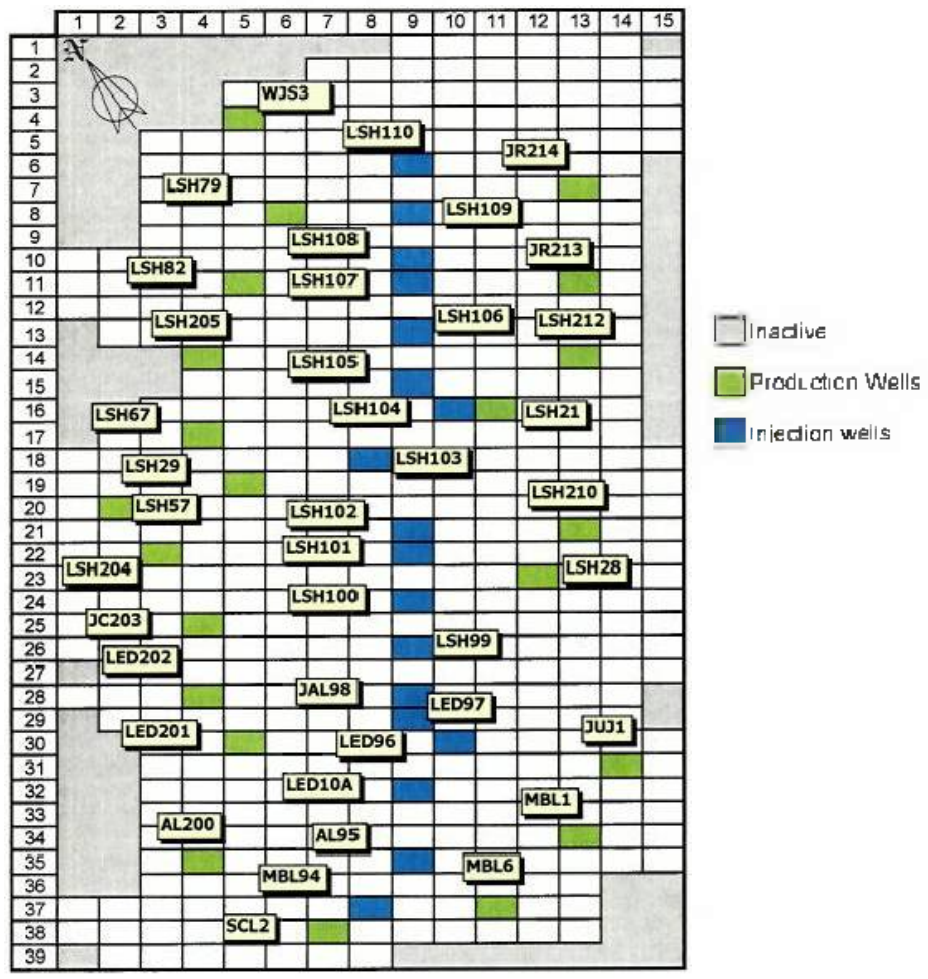


Figure 2.4.7 Wileyville field, location of the active production and injection wells

The latest production from this field, using secondary recovery, started in January 2000 with five production wells. During the next 18 months, the remaining producing wells were put on stream. The data from the field covers production to May 2002. At this point the field had produced $30 \mathrm{Mbbl}$ of oil and $21 \mathrm{Mbbl}$ of water, reflecting an increase in production during the last 10 months of $26 \mathrm{Mbbl}$ of oil and $14 \mathrm{Mbbl}$ of water (see Figure 2.4.8). This increase is attributed mainly to 2 factors - the completion of all the production wells and the arriving of the oil bank front at the production wells.

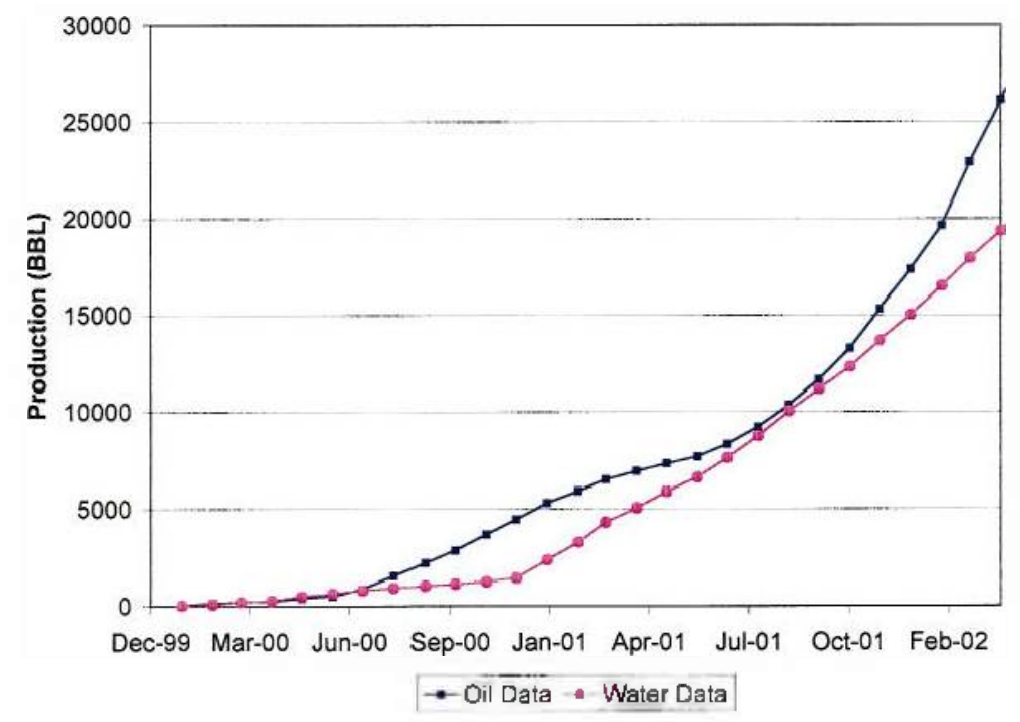

Figure 2.4.8. Wileyville field, cumulative water and oil production

During Phase I, of a reservoir model was developed. An important stage in the completion of a reservair modeling study is the creation of a discrete model. The discrete model is the representation of the reservoir characteristics for input into the computer model.

The discrete model represents a numerical form of the characteristics that resemble the reservoir properties. The representation of the reservoir properties is performed through different techniques. A block-centered grid is adopted in this study since the simulator used handles this type of grid. The property assigned to the center of the each block of the mesh is the singularity of this grid system. 
To create the discrete model the basic properties that must be known are the properties present in the Darcy's equation in porous media and Peaceman's equation. These properties include:

- Porosity distribution;

- Depth to top of the structure;

- Permeability distribution;

- Pay thickness of the sand; and

- Fluids saturation distribution (oil, ga,s and water).

An index matrix was generated for each of the fields in this study. This matrix establishes the blocks or cells that belong to the grid representing the reservoir. In order to create the index matrix, all of the properties of the reservoir have to be put together and the best representation of the reservoir boundaries determined. Considerations such as sand pinch-out, level of porosity, well locations, and preference of the flow in terms of direction were the most important characteristics necessary to address this issue.

The coordinate system chosen to develop the grid was rectangular coordinates and the orientation of the grid was marked by the trend of the injection line-drive. A twodimensional system was used since the formation thickness averages 10 to 12 feet. This permitted representation of the fields by a single-layer model.

The grid system for Wileyville field is based on an index matrix developed under the premise of non-uniform grid block size system. The non-uniform grid consists of grid blocks of different sizes, which allows refinement for locations of features such as production, injection or monitoring wells.

The number of blocks in the grid system is 646 blocks. Of these 646 blocks: 498 blocks represent the active cells. These active cells contain the producing part of the reservoir as well as the locations of the production and injection wells. The remaining 148 grid blocks represent the inactive part of the reservoir. The closed boundary is handled through the limit established between the active and inactive grid blocks of the index matrix. Figure 2.4.7 illustrates the index matrix in which active and inactive zones and well locations are established. Tables 2.4.1 and 2.4.2 show the names and locations for the 18 injection wells and the 22 production wells in the Wileyville field, respectively. 
Table 2.4.1 Injection wells and their location on the grid of the Wileyville field

\begin{tabular}{|l|c|c|c|}
\hline \multirow{2}{*}{ Farm } & \multirow{2}{*}{ Code No. } & Locat \\
\cline { 3 - 4 } & & X & Y \\
\hline L.S. Hoyt & LSH 110 & 9 & 6 \\
\hline L.S. Hoyt & LSH 109 & 9 & 8 \\
\hline L.S. Hoyt & LSH 108 & 9 & 10 \\
\hline L.S. Hoyt & LSH 107 & 9 & 11 \\
\hline L.S. Hoyt & LSH 106 & 9 & 13 \\
\hline L.S. Hoyt & LSH 105 & 9 & 15 \\
\hline L.S. Hoyt & LSH 104 & 10 & 16 \\
\hline L.S. Hoyt & LSH 103 & 8 & 18 \\
\hline L.S. Hoyt & LSH 102 & 9 & 21 \\
\hline
\end{tabular}

\begin{tabular}{|l|c|c|c|}
\hline \multicolumn{1}{|c|}{ Farm } & Code No. & Locat \\
\cline { 3 - 4 } & & X & Y \\
\hline L.S. Hoyt & LSH 101 & 9 & 22 \\
\hline L.S. Hoyt & LSH 100 & 9 & 24 \\
\hline L.S. Hoyt & LSH 99 & 9 & 26 \\
\hline Jacob A. Lantz & JAL 98 & 9 & 28 \\
\hline Louise E. Dulaney & LED 97 & 9 & 29 \\
\hline Louise E. Dulaney & LED 96 & 10 & 30 \\
\hline Louise E. Dulaney & LED 10A & 9 & 32 \\
\hline Ara Long & AL 95 & 9 & 35 \\
\hline Mary B. Long & MBL 94 & 8 & 37 \\
\hline \multicolumn{4}{|c|}{ 18 Injection wells } \\
\cline { 2 - 4 }
\end{tabular}

Table2.4.2 Production wells and their location on the grid of the Wileyville field

\begin{tabular}{|l|c|c|c|}
\hline \multicolumn{1}{|c|}{ Farm } & \multirow{2}{*}{ Code No. } & \multicolumn{2}{|c|}{ Locat. } \\
\cline { 3 - 4 } & & X & Y \\
\hline W.J. Santee & WJS 3 & 5 & 4 \\
\hline L.S. Hoyt & LSH 79 & 6 & 8 \\
\hline L.S. Hoyt & LSH 82 & 5 & 11 \\
\hline L.S. Hoyt & LSH 205 & 4 & 14 \\
\hline L.S. Hoyt & LSH 67 & 4 & 17 \\
\hline L.S. Hoyt & LSH 29 & 5 & 19 \\
\hline L.S. Hoyt & LSH 57 & 2 & 20 \\
\hline L.S. Hoyt & LSH 204 & 3 & 22 \\
\hline Jennetta Chamberlai & JC 203 & 4 & 25 \\
\hline Louise E. Dulaney & LED 202 & 4 & 28 \\
\hline Louise E. Dulaney & LED 201 & 5 & 30 \\
\hline
\end{tabular}

\begin{tabular}{|l|c|c|c|}
\hline \multirow{2}{*}{ Farm } & \multirow{2}{*}{ Code No. } & \multicolumn{2}{|c|}{ Locat. } \\
\cline { 3 - 4 } & & $X$ & $Y$ \\
\hline Ara Long & AL 200 & 4 & 35 \\
\hline Sarah C. Long & SCL 2 & 7 & 38 \\
\hline Mary B. Long & MBL 6 & 11 & 37 \\
\hline Mary B. Long & MBL 1 & 13 & 34 \\
\hline J. U. Jaliff & JUJ 1 & 14 & 31 \\
\hline L.S. Hoyt & LSH 28 & 12 & 23 \\
\hline L.S. Hoyt & LSH 210 & 13 & 21 \\
\hline L.S. Hoyt & LSH 21 & 11 & 16 \\
\hline L.S. Hoyt & LSH 212 & 13 & 14 \\
\hline Jonh Rush & JR 213 & 13 & 11 \\
\hline Jonh Rush & JR 214 & 13 & 7 \\
\hline \multirow{2}{*}{} & 22 Production wells \\
\cline { 3 - 4 } & \multicolumn{3}{|c}{}
\end{tabular}

\subsubsection{Near-Wellbore Remediation in Stripper Wells}

Near-wellbore damage remediation has direct impact on the improvement of the productivity of wells. This study investigated the potential impact of near-wellbore damage remediation on the production of shallow oil wells penetrating the Glade sandstone, located in Warren County, PA. Given the number of shallow reservoirs in the Appalachian and mid-continent basins, understanding of the potential for productivity 
enhancement in the Glade can provide valuable insight regarding the efficacy of the technology in shallow basins.

To accomplish this objective, a characterization of the reservoir was first developed using the CMG commercial simulator. History matching of the production data was used in this characterization study. The simulator was then modified using the rock and fluid properties determined during laboratory analysis of the core and fluids obtained from a well penetrating the Glade sandstone. Comparison of the results of these two studies was then used to ascertain the impact of the implementation of near-wellbore damage remediation on improving well performance. In addition an investigation of the reservoir behavior given re-pressurization was performed as an alternative to the near-wellbore damage remediation.

Our results show that removal of the formation damage would be an inefficient method of enhancing oil recovery for this particular oil field given its low reservoir pressure. By contrast, re-pressurization of the reservoir with $\mathrm{CH}_{4}$ showed positive results on the production from the wells penetrating the Glade sandstone. It was determined that the repressurization of the reservoir to a pressure approaching the freshwater gradient with the near-wellbore damage eliminated, would result in the highest increase in cumulative production. Complete description of the methods and results of this section can be found in the work of Gitman \& Watson |2005|. 


\subsection{References}

Bear, J. and Bachmat, Y. (1990) Introduction to Modeling of Transport Phenomena in Porous Media, Kluwer Publishing. Amsterdam.

Biot, M.A. (1956) Theory of Propagation of Elastic Waves in Fluid-Saturated Porous Solid, Journal of Acoustic Society of America, vol. 28., pp. $168-191$.

Corapcioglu, M.Y. (1991) Wave Propagation in Porous Media - A Review, Transport Processes in Porous Media (Editors Y. Bear and M.Y. Corapcioglu), pp. 373 469, Kluwer Publishing. Amsterdam.

Gitman, A. and Watson, R. W. (2005) Near-Wellbore Damage Remediation in Stripper Wells, SPE 97980, Presented at the 2005 SPE Eastern Regional Meeting held in Morgantown, W.V., September 2005.

Gollapudi, U. K., Bang, S. S. and M. R. Isalm (1994) Ultrasonic treatment for removal of Asphaltene deposits during petroleum production. SPE 27377, 653-660.

Levy, A., Ben-Dor, G., and Sorek, S. (1996) Numerical Investigation of the Propagation of Shock Waves in Rigid Porous Materials - Development of the Computer Code and Comparison with Experimental Results. Journal of Fluid Mechanics, vol. 3-4, pp. $163-179$.

Ozdemir, M. (2004) Effect of Ultrasonic Radiation on Rock Permeability, M.S. Thesis, The Pennsylvania State University, August 2004.

Poesio, P., Ooms, G., and Barake, S. (2002) An investigation of the influence of acoustic waves on the liquid flow through a porous material, Journal of Acoustic Society of America, vol. 111, 2019-2025.

Quammie, K.E. (2004) Numerical Modeling of Cavitation Phenomenon in Flowing Fluids, M.S. Thesis, The Pennsylvania State University, May 2004.

Rejepov, D.M. (2004) A Qualitative Analysis of Near-Wellbore Thermal Field Generated by Acoustic Waves, M.S. Thesis, The Pennsylvania State University, May 2004.

Venitaraman, A., Roberts, P. M. and Sharma, M. M. (1994) Ultrasonic removal of nearwellbore damage caused by fines and mud solids. SPE 27388, 441-448. 


\subsection{Conclusions}

The objective of this project was to develop a sonic well performance enhancement technology that focused on near wellbore formations. In order to successfully achieve this objective, a three-year (i.e., three phase) project was defined

with each year consisting of four tasks. The first task was a laboratory-scale study with the goal of determining the underlying principles of the technology. A scale-up mathematical model was to be developed in the second task to serve as the design guide for tool development. The third task was the development of effective transducers that can operate with variable frequency so that the most effective frequencies can be applied in any given situation. The system, assembled as part of the production string, was to ensure delivery of sufficient sonic energy to penetrate the near-wellbore formation. The last task was the actual field testing of the tool.

The first phase of the project has been completed, which was performed over a two-year period. The overall goals of the project were not met as the project was terminated after performing only the first of three phases; however, the objectives of the first phase were met and are summarized by task. Laboratory-scale testing and modeling was performed and baseline field testing was conducted. The testing and modeling did not proceed to the point, however, where the final tool could be designed, constructed, and tested in the field since two phases of activities were not performed..

\section{Task 1. Laboratory-Scale Experiments}

In Task 1, a literature review of laboratory and field investigations employing acoustic stimulation to enhance fluid flow in porous media was performed. Two characteristics appear to be common to most of the previous work reported. One is lack of systematic data that may be truly classified as scientific, and the other, is inconsistency the empirical evidence offers as to the impact of elastic waves on oil recovery from petroleum reservoirs.

The findings from the literature review were used to design our experimental protocol. A slim-tube experimental system was designed to enable us to perform systematic studies on the interaction of elastic waves with fluids in porous media. 
Experiments performed during the first phase focused on determining the inception of cavitation, studying thermal dissipation under cavitation conditions, investigating sonic energy interactions with glass beads and oil, and studying the effects of sonication on crude oil properties.

Our initial focus was on determining the operating characteristics of transducers with the goals of delineating the conditions for the onset of cavitation and determining the power dissipation rate during cavitation since we believe that sonic stimulation will be enhanced when the device is operated under cavitation conditions. Our findings show that the voltage threshold for onset of cavitation is independent of transducer-hydrophone separation distance.

Thermal dissipation under cavitation conditions was studied because energy dissipation may promote the mobilization of deposited paraffins and waxes. Our preliminary laboratory experiments suggest that waxes are mobilized when the fluid temperature approaches $40^{\circ} \mathrm{C}$.

Studies were performed investigating the interaction of sonic energy with glass beads in water, with oil droplets in water, and core samples. The tests with glass beads and oil in water provided phenomenal information with respect to movement, coalescence, and effect on composition. Acoustic energy drove off the lighter fractions, aromatics, and branched alkanes.

\section{Task 2. Development of a Computer Model for Scale-Up}

The primary efforts towards simulating of the effect of sonication on the nearwellbore region have focused on the development of a numerical model for handling the transportation of fluids under the influence of strong sonic waves in the porous medium. Governing equations describing this type of fluid transport process have not been adequately presented in the literature, and hence significant effort was directed towards this effort in Task 2. Activities included developing the numerical framework, developing accurate algorithm solutions, and validating the code. 


\section{Task 3. Development of a New Generation of Acoustic Transducers}

Past systematic investigations of flow-enhanced parameters are poorly defined and irreproducible. The reproducibility of these experiments may be difficult, if not impossible, due to insufficient information. The need to understand the governing mechanisms of acoustic energy-fluid-solid interactions in porous media requires versatile transducers that can be operated within various parameters. In this task, experiments were performed to gain insight into these interactions and provide information to design a slim-tube apparatus for performing the experiments. The design of the slim-tube apparatus was performed during the first phase of the project. Results from testing that was to be performed using the slim-tube apparatus during the second phase was then to be used to design and buildthe tool for field testing.

\section{Task 4. Field Testing of the Sonication Technolagy}

The overall plan for this task was to perform field trials of the sonic transducer design. The trials were to be performed in production and/or injection wells located in Pennsylvania, New York, and West Virginia. Work during the first phase included performing literature searches, meeting with producers to schedule testing, gathering production information, cores, and samples from wells, and drilling four new wells for use in the project. Field testing of the tool was to be performed in subsequent phases. 


\subsection{Acknowledgments}

Funding for this work was provided by the U.S. Department of Energy and The Pennsylvania State University under Cooperative Agreement No. DE-FC26-02NT15187. The project was managed by the U.S. Department of Energy, National Energy Technology Laboratory. Ms. Nancy Comstock from DOE's National Petroleum Technology Office was the Contracting Officer's Representative.

The following individuals and companies are acknowledged for their assistance in the project. Messrs. Louie, Gerry, and Gilbert Geer of IL Geer \& Sons, Clarendon, Pennsylvania, are acknowledged for drilling four new wells for the project and providing cores for testing as well as samples of oil, brine, frac sand, and paraffin. Mr. Eric Gray of Utilities \& Industries, Inc., Reynoldsville, Pennsylvania, is acknowledged for providing four meters to monitor the newly drilled wells. Mr. John Holko of Lenape Resources, Batavia, New York, provided well information. Mr. Robert Metzgar of Metzgar Oi] Company and North Penn Pipe \& Supply, Warren, Pennsylvania, is acknowledged for providing an existing well, information, and piping supplies. Messrs. William Fustos, Paul Dudenus, and Terry Pegula of East Resources, Cranberry, Pennsylvania, provided well information. 
Appendix A. Literature Review of the Influence of Acoustic Energy on Permeability of Porous Media 
Table A. I Laboratory studies of the influence of acoustic energy on permeability of porous media

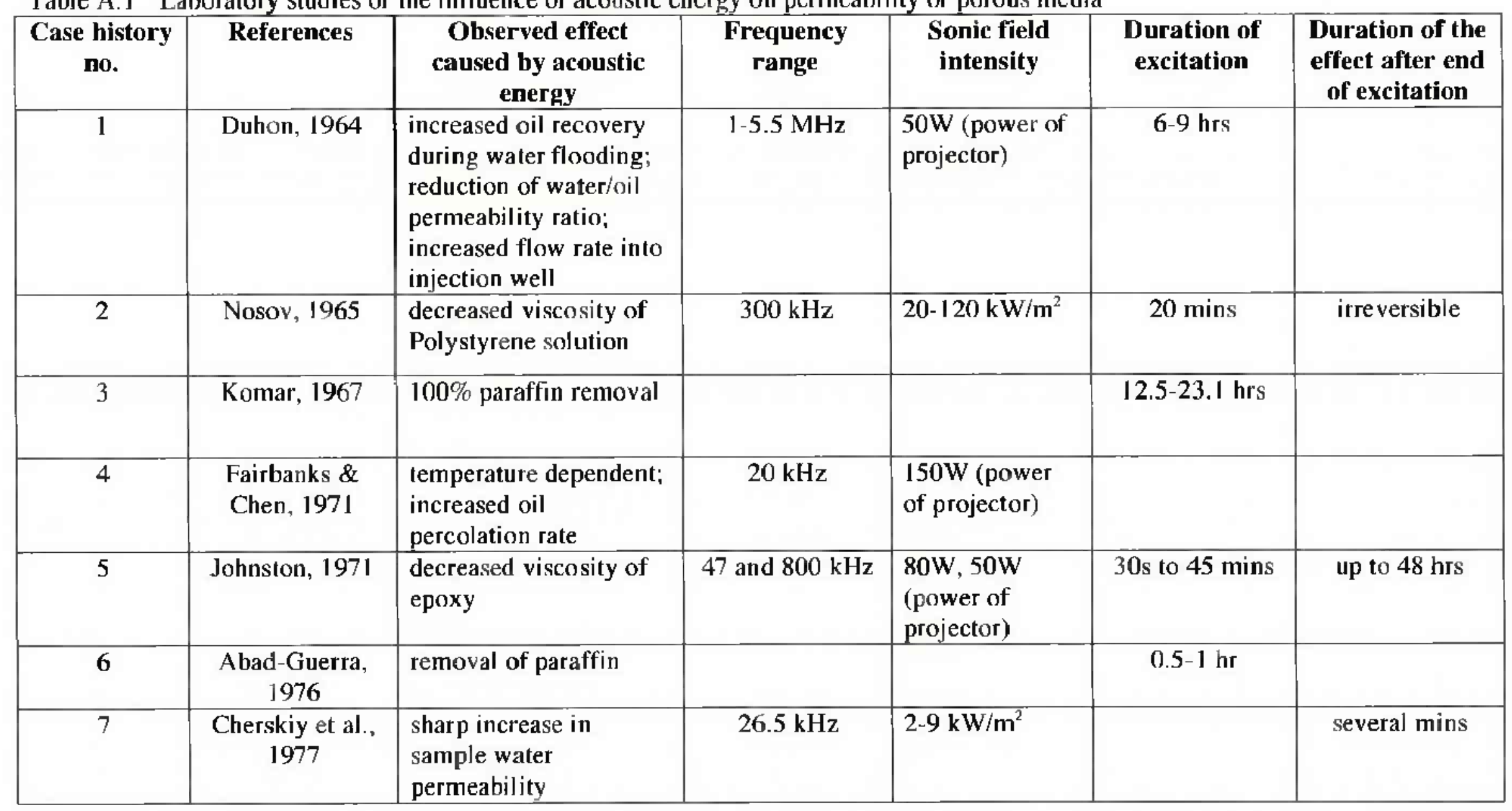




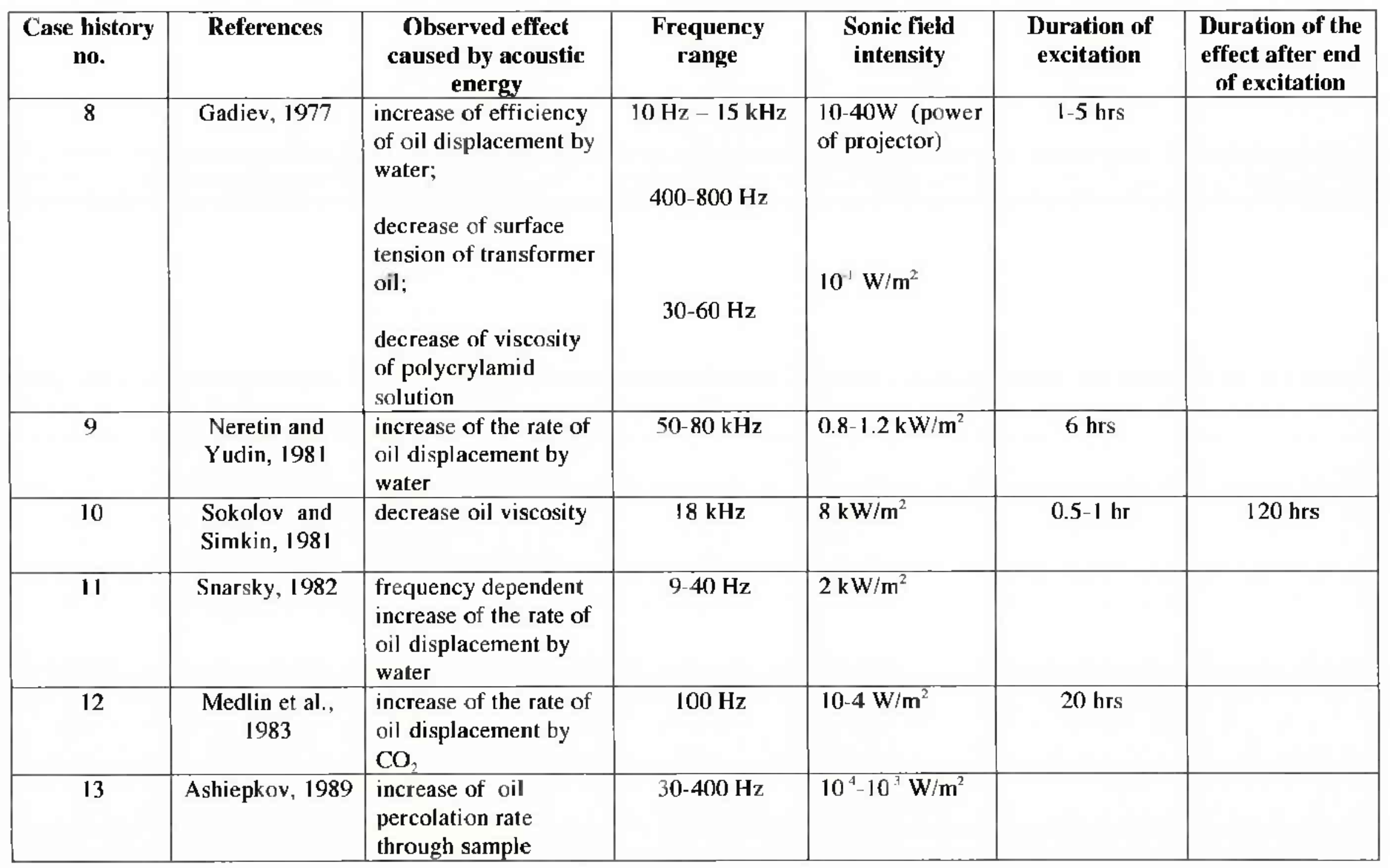




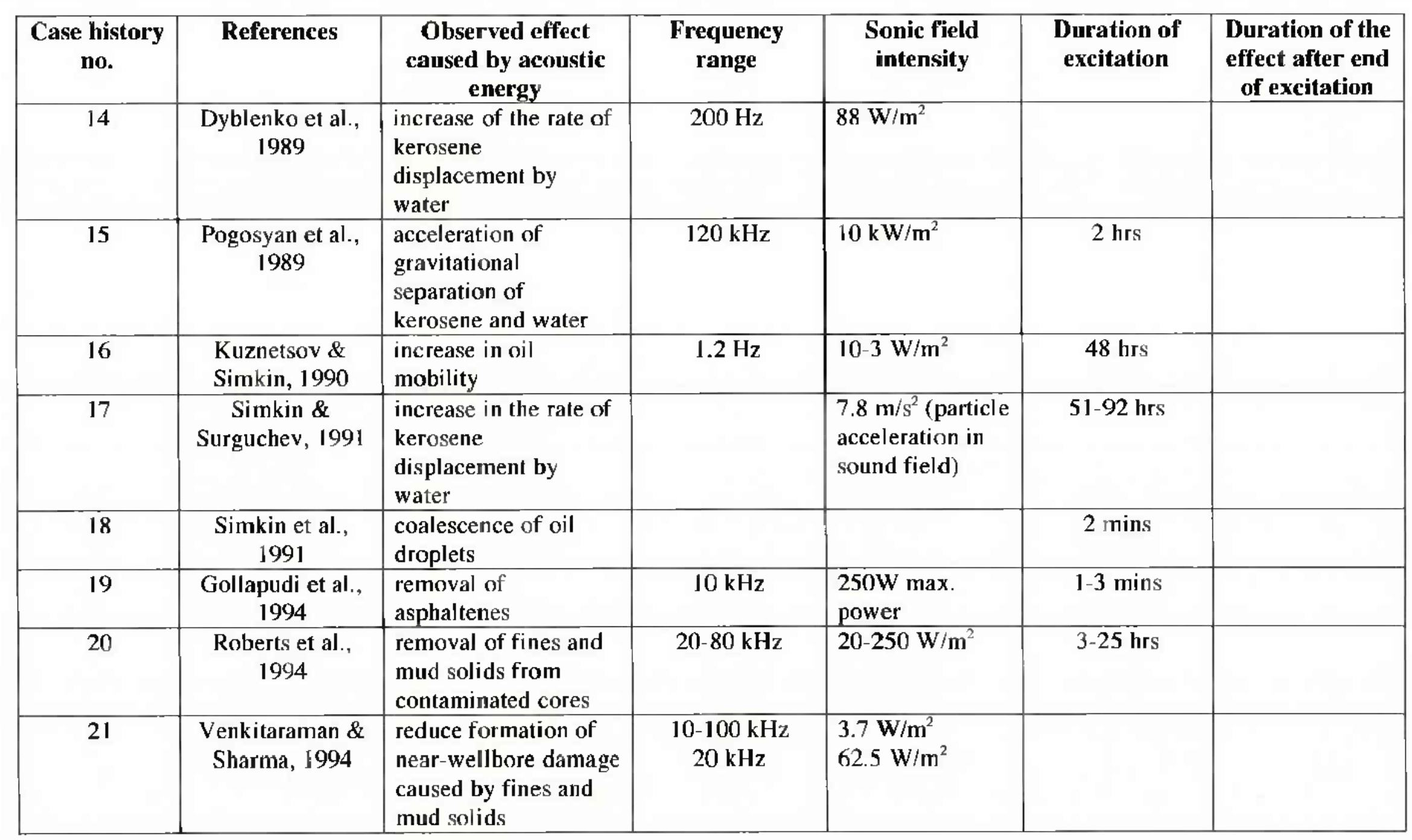




\begin{tabular}{|c|c|c|c|c|c|c|}
\hline $\begin{array}{c}\text { Case history } \\
\text { no. }\end{array}$ & References & $\begin{array}{l}\text { Observed effect } \\
\text { caused by } \\
\text { acoustic energy }\end{array}$ & $\begin{array}{l}\text { Frequency } \\
\text { range }\end{array}$ & $\begin{array}{l}\text { Sonic field } \\
\text { intensity }\end{array}$ & $\begin{array}{c}\text { Duration of } \\
\text { excitation }\end{array}$ & $\begin{array}{l}\text { Duration of the } \\
\text { effect after end } \\
\text { of excitation }\end{array}$ \\
\hline 22 & $\begin{array}{c}\text { Wilkey et al., } \\
1999\end{array}$ & $\begin{array}{l}\mathrm{BaSO}_{4} \text { scale } \\
\text { removal }>90 \% \\
\text { success }\end{array}$ & $20 \mathrm{kHz}$ & $20 \mathrm{~W} / \mathrm{m}^{2}$ & $15 \mathrm{mins}$ & \\
\hline 23 & $\begin{array}{c}\text { Roberts et al., } \\
2000\end{array}$ & $\begin{array}{l}\text { removal of } \\
\text { paraffin }\end{array}$ & $20-36 \mathrm{kHz}$ & $100-1,800 \mathrm{~W} / \mathrm{m}^{2}$ & $2.4 \mathrm{hrs}$ & \\
\hline 24 & $\begin{array}{l}\text { Poesio et al., } \\
2002\end{array}$ & $\begin{array}{l}\text { decreased } \\
\text { pressure } \\
\text { gradient; } \\
\text { permeability } \\
\text { remained } \\
\text { constant }\end{array}$ & 20 and $40 \mathrm{kHz}$ & 2 and $0.7 \mathrm{~kW}$ & & \\
\hline 25 & Lin \& Yen 1993 & $\begin{array}{l}\text { Asphaltene } \\
\text { dispersion in } \\
\text { surfactants }\end{array}$ & & $\begin{array}{l}6 \mathrm{~W} \text { (projector } \\
\text { max. power) }\end{array}$ & & \\
\hline 26 & $\begin{array}{c}\text { Gunal \& Islam } \\
2000\end{array}$ & $\begin{array}{l}\text { Decreased oil } \\
\text { viscosity in } \\
\text { water- } \\
\text { asphaltene } \\
\text { solution }\end{array}$ & $10 \mathrm{kHz}$ & $\begin{array}{l}250 \mathrm{~W} \text { (projector } \\
\text { power) }\end{array}$ & & \\
\hline 27 & Shedid 2002 & $\begin{array}{l}\text { Increased } \\
\text { permeability of } \\
\text { asphaltene } \\
\text { damaged core; } \\
\text { decreased oil } \\
\text { viscosity with } \\
\text { solvent addition }\end{array}$ & $\begin{array}{c}10,15, \text { and } 20 \\
\mathrm{kHz}\end{array}$ & & 5 to 30 minutes & \\
\hline
\end{tabular}




\section{References}

Abad-Gerra, B. P. (1976) Methods for restoring productivity to gas wells in the Clinton sand of Ohio: A laboratory and field experiment: Ph.D. thesis, Pennsylvania State University.

Ashiekov, J. S., Riashentsev, N. P. and Cherednikov, E. N. (1989) Controllable viroseismic action- A new method of the production stimulation. In Numerical methods of percolation problem analysis. Dynamic of multiphase media (Chislenniye metody resheniya zadach filtratsiii. Dinamika mnogofaznikh sred); 8-22 (in Russian).

Cherskiy, N. V., Tsarev, V. P., Konovalov, V. M. and Kuznetsov, O. L. (1977) The effects of ultrasound on permegbility of rocks to water. Transactions (Dklady) of the USSR Academy of Sciences, Earth Science Sections, vol. 232, 201-204.

Dyblenko, V. T., Tufanov, I. A. Suleymanov, G. A. and Lysenkov, A. P. (1989) Percolation phenomena and processes in saturated porous media under the vibro-wave action. In Ways of intesification of oil production (Puty intensifikatsii dobychi nefti), Proc. (Trudy) Bashkir Research and Design Institute of Oil (BASHNIPINEFT): $45-51$ (in Russian).

Fairbank, H. V. and Chen, W. I. (1971) Ultrasonic acceleration of liquid flow through porous media. Chemical Engineering Progress Symposium Ser., vol. 67, 108-116.

Gadiev, S. M. (1977) Use of vibrations in oil production (Ispol'zonvaniye vibratsii v dobyche nefti), Nedra Ptess (in Russian).

Gollapudi, U. K., Bang, S. S. and M. R. Isalm (1994) Ultrasonic treatment for removal of Asphaltene deposits during petroleum production. SPE 27377, 653-660.

Gunal, O. G. and Islam, M. R. (2000) Alteration of asphaltic crude rheology with electromagnetic and ultrasonic irradiation. Journal of Petroleum Science and Engineering, vol. 26, 263-272.Bureau, New York.

Johnston, H. K. II (1971) Polymer viscosity control by use of ultrasonics. Chemical Engineering Progress Symposium Ser., vol. 67, 39-45.

Komar, C. A. (1967) Effects of ultrasonics on Appalachian paraffin. Petroleum Engineering, vol. $39,437-440$.

Kuznetsov, O. L. and Simkin, E. M. (1990) Transformation and interaction of geophysical fields in lithosphere (Preobrazovaniye i vzaimodeystviye geofizicheskikh poley v litosfere); 106-112. Rock permeability for water and oil, oil viscosity, capillary pressure under the influence of elastic waves; Sec. 3.4, Effects of elastic wavefield; Sec. 4.4, Fluids separation in elastic wavefield; Nedra Press (in Russian).

Lin, J-R, and Yen, T. F. (1993) Upgrading process through cavitation and surfactant. Energy \& Fuels, v 7, no. 1, Jan-Feb, 1993, 111-118. 
Medlin, W. L., Masse, L. and Zumwalt, G. L. (1983) Methods for recovery of oil by means of a gas drive combined with low amplitude seismic excitation. U.S.A. Patent 4417621.

Neretin, V. D. and Yudin, V. A. (1981) Results of experimental studies of the influence of acoustic treatment on percolation processes in saturated porous media In Topics in nonlinear geophisics (voprosi nelineinoy geofiziki): All-Union Research Institute of Nuclear Geophysics and Geochemistry (VNIIYaGG), 132-137 (in Russian).

Nosov, V. A. (1965) Soviet progress in applied ultrasonics, vol. 2: Ultrasonics in the chemical industry: Consultant Bureau, N.Y.

Progosyan, A. B., Simkin, E. M., Stremovskiy, E. V., Surguchev, M. L. and Shnirel'man, A. I. (1989) Separation of hydrocarbon fluid and water in an elastic wavefield acting on a porous reservoir medium. Transactions (Doklady) of the USSR Academy of Sciences, Earth Science Sections, vol. 307, 575-577.

Poesio, P., Ooms, G., and Barake, S. (2002) An investigation of the influence of acoustic waves on the liquid flow through a porous material, Journal of Acoustic Society of America, vol. $111,2019-2025$.

Roberts, P.M., Venkitarman, A. and Sharma, M.M. (2000) Ultrasonic removal of organic deposits and polymer-induced formation damage. Society of Petroleum Engineering and Completion, vol. 15, no. 1, 19-34.

Shedid, S. A. (2002) A novel technique of asphaltene deposition treatment using ultrasonic irradiation. Petroleum Science and Technology, vol. 20, n 9-10, October/November, 2002, 1097-1118.

Simkin, E. M., Efinova, S. A., Kuznetsov, O. L., Pechkov, A. A., ZhuyKov. J. S., Driagin, V. V., Rafikov, R. S. and Sarksiants, B. R. (1990) Creation and industrial realization of the technology of controllable acoustic treatment for mineral production stimulation in wells (a report): Krylov Research Institute of Oil and Gas (VNII) (in Russian).

Simkin. E. M., and Surguchev, M. L. (1991) Advanced vibroseismic techniques for water flooded reservoir stimulation. Mechanism and field results. Proceedings $6^{\text {th }}$ European Symposium on Improved Oil Recovery (Stavanger, Norway), 1, Book 1, 233-241.

Simkin. E. M., Surguchev, M. L., Akhapkin, M. J. Pogosyan, A. B. and Stupochenko, V. E. (1991) Advanced vibroseismic techniques for water flooded reservoir stimulation. Mechanisms and field results. Proc. $6^{\text {th }}$ Europ. Symp. On Improved Oil Recovery (Stavanger, Norway), 1, Book 1, 233-241.

Snarskiy, A. N. (1982) Determination of the influence of ultrasonic filed on oil percolation rate in elementary reservoir model. Transactions of the Higher School Izvestiia Vysshikh Uchebnikh Zavedennii), Ser, Oil and Gas (Neft' I Gaz), no. 1, 30-32. 
Sokolov, A. V. and Simkin, E. M. (1981) Study of influence of acoustic treatment on rheological properties of some oils. In Topics in nonlinear geophisics (voprosi nelineinoy geofiziki): All-Union Research Institute of Nuclear Geophysics and Geochemistry (VNIIYaGG), 137-142 (in Russian).

Venitaraman, A., Roberts, P. M. and Sharma, M. M. (1994) Ultrasonic removal of near-wellbore damage caused by fines and mud solids. SPE 27388, 441-448.

Wilkey, M. L., Peters, R. W., and Furness Jr., J. C. (1999) The use of advanced acoustic cavitation for applications in the oil and natural gas industry. The 1999 Oil \& Gas Conferfence cosponsored by the U.S. Department of Energy, Federal Energy Technology Center (FETC) and National Petroleum Technology Office (NPTO), June 28-30, Dallas, Texas. 


\section{Appendix B. Details of Model Derivations}

\section{Appendix B.1 Derivation of the Averaging Rules}

For the derivations below we consider the REV that contains two phases $\alpha$ and $\beta$ which occupies a domain $u_{o}$ and has a volume $U_{o}$. This REV is sketched below:

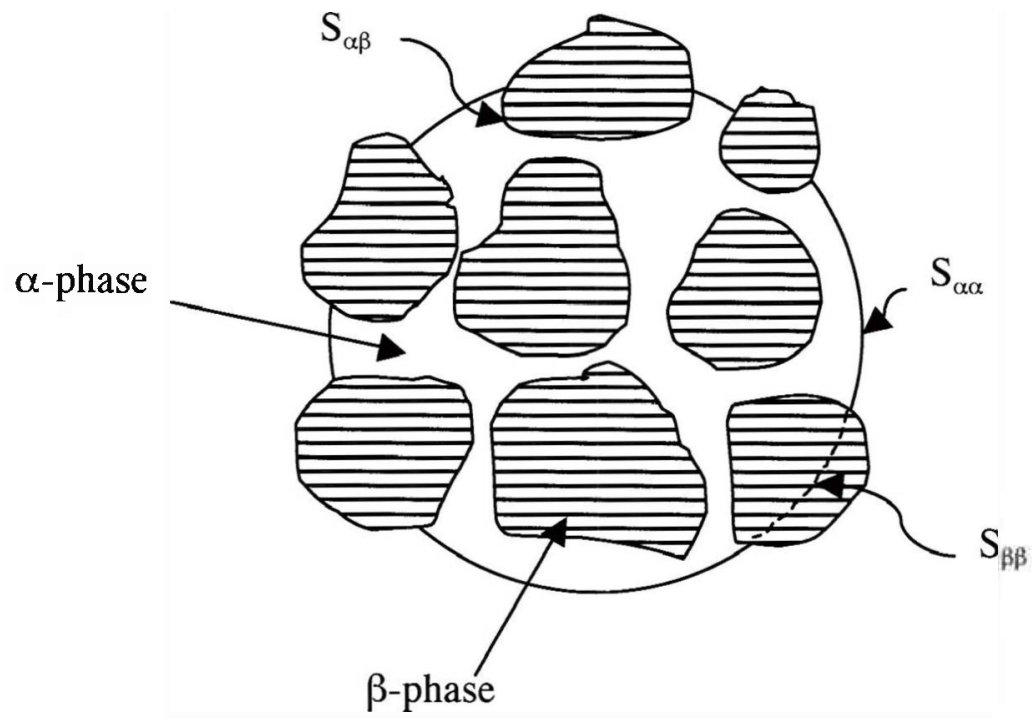

Figure 1. Schematic of a Representative Elementary Volume showing the inter-phase surfaces. 1. The sum of two quantities:

Let $f\left(x^{\prime}, t\right)$ and $g\left(x^{\prime}, t\right)$ be two quantities related to certain phase $\alpha$, and let $\bar{f}$ and $\bar{g}$ be the volume average of these quantities with respect to $\alpha$, that is:

$$
\bar{f}=\frac{1}{U_{o \alpha}} \int_{u_{o \alpha}} f d U
$$

And

$\bar{g}=\frac{1}{U_{o \alpha}} \int_{u_{o \alpha}} g d U$

where $U_{o \alpha}$ is the volume occupied by the $\alpha$ phase then:

$\overline{f+g}=\frac{1}{U_{o \alpha}} \int_{u_{o \alpha}}(f+g) d U=\frac{1}{U_{o \alpha}} \int_{u_{o \alpha}} f d U+\frac{1}{U_{o o r}} \int_{u_{o \alpha}} g d U$

therefore:

$\overline{f+g}=\bar{f}+\bar{g}$ 


\section{The average of a product:}

We start by denoting the deviation from the average $\bar{f}$ value of any property $f$ by $f^{\prime}$. We note that the average property is associated with the location vector $\vec{x}$ of the centroid of the REV and we define the deviation term as follows:

$f\left(x^{\prime}, t\right)=\overline{f(x, t)}+f^{\prime}\left(x^{\prime}, t\right)$

and similarly we can write:

$g\left(x^{\prime}, t\right)=\overline{g(x, t)}+g^{\prime}\left(x^{\prime}, t\right)$

We can now proceed to determine the average of a product as follows:

$\overline{f g}=\frac{1}{U_{o \alpha}} \int_{U} f g d U=\frac{1}{U_{o \alpha}} \int_{u_{o \alpha}}\left(\bar{f}+f^{\prime}\right)\left(\bar{g}+g^{\prime}\right) d U=\frac{1}{U_{o \alpha}} \overline{f g} \int_{u_{o \alpha}} d U+\bar{f} \frac{1}{U_{o \alpha}} \int_{u_{o \alpha \alpha}} g d U$

$+\bar{g} \frac{1}{U_{o \alpha}} \int_{u_{o \alpha}} g^{\prime} d U+\frac{1}{U_{o \alpha}} \int_{u_{o \alpha}} f^{\prime} g^{\prime} d U$

where $u_{o \alpha}$ is the

domain of the $\alpha$ phase within the REV. We note that the average of a deviation term about the centroid of the REV is zero (since this is how the centroid is defined), and with this we can reduce the previous equation to:

$\overline{f g}=\bar{f} \bar{g}+\overline{f^{\prime} g^{\prime}}$

\section{Average of a time derivative:}

We begin by applying Reynolds' transport theorem to the density $e$ of an extensive property $E$ of the $\alpha$ phase:

$\frac{D_{E}}{D t} \int_{u_{\alpha \alpha x}(t)} e d U=\int_{u_{o \alpha}(t)} \frac{\partial e}{\partial t} d U+\int_{S_{\alpha \beta}(t)} e u \cdot n d S+\int_{S_{\alpha \alpha}(t)} e V^{E} \bullet n d S$

where:

- $u$ is the displacement velocity of the surface $S_{\alpha \beta}$,

- $n$ is the outward normal unit vector from the surface of integration,

- and $V^{E}$ is the velocity of the $\alpha$ continuum.

We can also look at the material rate of change of $\alpha$ within the whole REV. This change can be expressed by:

$\frac{D_{E}}{D t} \int_{u_{o}} \gamma_{\alpha} e d U=\frac{\partial}{\partial t} \int_{u_{o}} \gamma_{\alpha} e d U+\int_{S_{o}} \gamma_{\alpha} e V^{E} \cdot n d S$

where:

$\gamma_{\alpha}(x)=1$ when $x$ is within $u_{o \alpha}$ and $\gamma_{\alpha}(x)=0$ otherwise.

And with the terms in equation (R.3.2) defined as follows:

$\frac{D_{E}}{D t} \int_{u_{o}} \gamma_{\alpha} e d U$ : The material rate of change of $\alpha$ within $u_{o}$,

$\frac{\partial}{\partial t} \int_{u_{o}} \gamma_{\alpha} e d U:$ The instantaneous rate of change of $\alpha$ within $u_{o}$, and

$\int_{S_{o}} \gamma_{\alpha x} e V^{E} \bullet n d S:$ The net rate of exchange of $E$ through the boundary surface $S_{o}=S_{\alpha \alpha}+S_{\beta \beta}$. 
We can rewrite (R.3.2) taking into account the definition of $\gamma_{\alpha}$ as follows:

$\frac{D_{E}}{D t} \int_{u_{o u}} e d U=\frac{\partial}{\partial t} \int_{u_{o u}} e d U+\int_{S_{c u t}} e V^{E} \cdot n d S$

Now by equating (R.3.1) and (R.3.3) and canceling terms that appear on both sides we obtain:

$\frac{\partial}{\partial t} \int_{u_{o \alpha}} e d U=\int_{u_{n \pi}(t)} \frac{\partial e}{\partial t} d U+\int_{S_{\alpha \beta}(t)} e u \cdot n d S$

Next we define the average of the any property $e$ with respect to the $\alpha$ phase as:

$\bar{e}=\frac{1}{U_{o \alpha}(x, t)} \int_{u_{o u}(x, t)} e\left(x^{\prime}, t ; x\right) d U$

where the notation $x^{\prime}, t ; x$ is to indicate that the location vector $x^{\prime}$ is within an REV whose centroid is associated with the vector $x$.

Similarly:

$$
\frac{\overline{\partial e}}{\partial t}=\frac{1}{U_{o \alpha}} \int_{u_{o \alpha}} \frac{\partial e}{\partial t} d U
$$

by substituting (R.3.4) and (R.3.5) into (R.3.3) we obtain the averaging rule for a time derivative:

$\frac{\partial}{\partial t}\left(U_{o \alpha} \bar{e}\right)=U_{o \alpha} \frac{\overline{\partial e}}{\partial t}+\int_{S_{\alpha \beta}} e u \cdot n d S$

we can further simplify (R.3) by dividing across by the volume of the REV $\left(U_{o}\right)$ to obtain:

$\frac{\partial}{\partial t}(\theta \bar{e})=\theta \frac{\overline{\partial e}}{\partial t}+\frac{1}{U_{n}} \int_{S_{a \beta}} e u \cdot n d S$

where $\theta$ is the phase saturation. We note here that for a fully saturated porous medium this is equal to the porosity therefore we can rewrite (R.3.6) as:

$\frac{\partial}{\partial t}(\phi \bar{e})=\phi \frac{\overline{\partial e}}{\partial t}+\frac{1}{U_{o}} \int_{S_{\alpha \bar{\beta}}} e u \cdot n d S$

\section{Average of a spatial derivative}

We begin by stating Gauss' theorem:

$\int_{u} \frac{\partial G_{j k l .}}{\partial x_{i}} d U=\int_{s} G_{j k l . .} n_{i} d S$

where $G_{j k l . . .}$ is a tensor of any rank and $n_{i}$ denotes the component of the outward normal unit vector, which is also known as a direction cosine, $u$ is a convex spatial domain and $S$ is the surface bounding it . Figure R.4.1 illustrates how the surface elements are calculated.

By applying Gauss's theorem to the $u_{o \alpha}$ domain we obtain:

$$
\int_{u_{o \alpha}} \frac{\partial G_{j k l \ldots \ldots}}{\partial x_{i}} d U=U_{o} \theta \frac{\overline{\partial G_{j k l \ldots}}}{\partial x_{i}}=\int_{S_{\alpha \beta}} G_{j k l \ldots .} \cos \left(n, 1 x_{i}\right) d S+\int_{S_{\alpha \alpha}} G_{j k l \ldots} \cos \left(n, 1 x_{i}\right) d S
$$




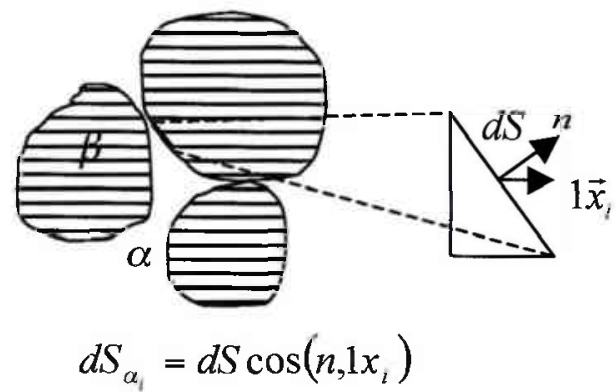

Figure R.4.1. A schematic showing how the surface elements are determined.

Now, we can write the equation for the instantaneous rate of change of the sum of $G_{j k l}$ over the domain $u_{o \alpha}$ as:

$\frac{\partial}{\partial x_{i}} \int_{u_{o \alpha}} G_{j k l . \ldots} d U=\frac{\partial}{\partial x_{i}}\left(U_{o} \theta \overline{G_{j k l \ldots}}\right)=U_{o} \theta \frac{\partial \overline{G_{j k l \ldots}}}{\partial x_{i}}+\overline{G_{j k l . .}} U_{o} \frac{\partial \theta}{\partial x_{i}}$

But this rate of change can also be evaluated using Figure R.4.2 as:

$$
\begin{aligned}
& \frac{\partial}{\partial x_{i}} \int_{u_{o x}} G_{j k l . .} d U=\lim _{\Delta x_{i} \rightarrow 0}\left(\int_{u_{o \alpha}(x+d x)} G_{j k l} d U-\int_{u_{o \alpha}} G_{j k l} d U\right) \\
& =\lim _{\Lambda x_{1} \rightarrow 0}\left\{\left(\int_{u_{o \alpha}, 2} G_{j k l . . .} d U+\int_{u_{o \alpha}, 3} G_{j k l . . .} d U\right)-\left(\int_{u_{o \alpha}, 1} G_{j k l, \ldots} d U+\int_{u_{o \alpha}, 2} G_{j k l . . .} d U\right)\right\} \\
& =\lim _{\Delta x_{i} \rightarrow 0}\left(\int_{u_{o g}, 3} G_{j k l \ldots} d U-\int_{u_{o a}, 1} G_{j k l} d U^{t}\right) \\
& =\Delta x_{i} \frac{1}{\Delta x_{i}}\left(\int_{(4,7,6)} G_{j k l} d S_{i}+\int_{(4,5,6)} G_{j k l} d S_{i}\right) \\
& =\int_{S_{\alpha \alpha}} G_{j k l . .} \cos \left(n, 1 x_{i}\right) d S
\end{aligned}
$$

We note here that the change in sign when the volume integrals in (R.4.4) where replaced with surface integrals is due to the directions of the normal unit vectors (See Figure R.4.2). Also the final surface integral is taken on the surface $S_{\alpha \alpha}$ because the surface element $d S_{i}$ is always located in the $\alpha$ phase (See Figure R.4.1).

Now we conclude our derivation by combining equations R.4.2 and R.4.4, i.e. :

$$
\int_{u_{o \alpha}} \frac{\partial G_{j k l \ldots}}{\partial x_{i}} d U=U_{o} \theta \frac{\overline{\partial G_{j k l \ldots}}}{\partial x_{i}}=\int_{S_{a \beta}} G_{j k l \ldots} \cos \left(n, 1 x_{i}\right) d S+\frac{\partial}{\partial x_{i}} \int_{u_{o \alpha}} G_{j k l \ldots} d U
$$




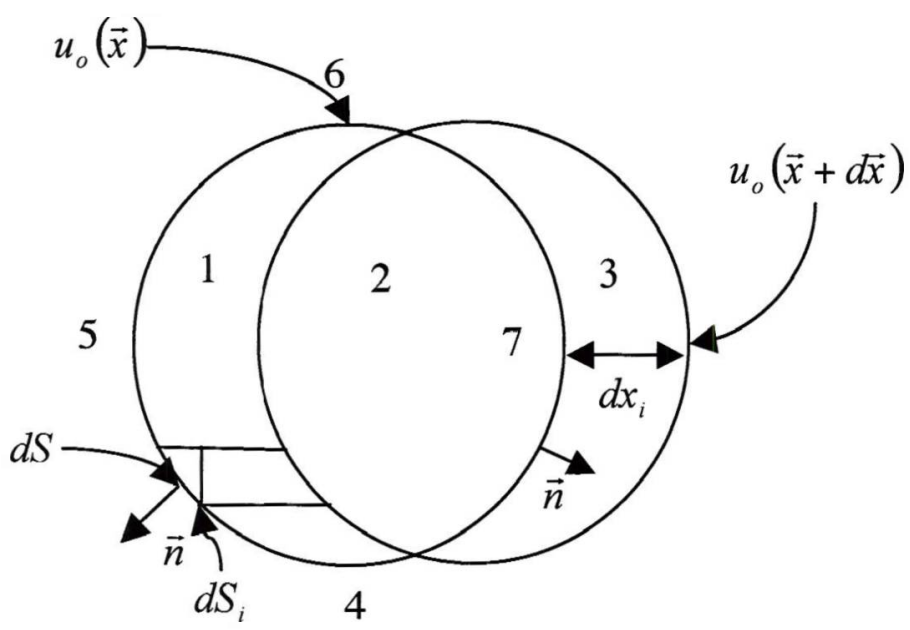

Figure R.4.2. A schematic corresponding to equation (R.4.4).

And from (R.4.5) and (R.4.3) we obtain the desired averaging rule:

$U_{o} \theta \frac{\overline{\partial G_{j k l}}}{\partial x_{i}}=\int_{s_{\alpha \beta}} G_{j k l \ldots} \cos \left(n, 1 x_{i}\right) d S+\frac{\partial}{\partial x_{i}}\left(U_{o} \theta \overline{G_{j k l} \ldots}\right)$

\section{Average of a spatial derivative of a scalar satisfying $\nabla^{2} G=0$}

Let $a_{j}=a_{j}(x)$ be a vector function of the position vector that is continuous and twice differentiable and let $b=b(x)$ b a scalar function of the position vector that is also continuous and twice differentiable. By first substituting $G_{j i}=b \frac{\partial a_{j}}{\partial x}$ into equation (R.4.1), Gauss' theorem, and expanding, and then letting $G_{j i}=a_{j} \frac{\partial b}{\partial x}$ and performing the same procedure and then subtracting the second expression from the first expression, we obtain the following equation (known as Green's vector theorem):

$\int_{S}\left(b \frac{\partial a_{j}}{\partial x_{i}}-a_{j} \frac{\partial b}{\partial x_{i}}\right) n_{i} d S=\int_{u}\left(b \frac{\partial^{2} a_{j}}{\partial x_{i} \partial x_{i}}-a_{j} \frac{\partial^{2} b}{\partial x_{i} \partial x_{i}}\right) d U$

Now, let $a_{j}=x_{j}^{\prime}$ and $b=G$ in the domain $u_{o \alpha}$, by substituting into (R.5.1) we obtain:

$\int_{S_{o \alpha}}\left(G \frac{\partial x_{j}^{\prime}}{\partial x_{i}}-x_{j}^{\prime}, \frac{\partial G}{\partial x_{i}}\right) n_{i} d S=\int_{u_{o \alpha}}\left(G \frac{\partial^{2} x_{j}^{\prime}}{\partial x_{i} \partial x_{i}}-x_{j}^{i} \frac{\partial^{2} G}{\partial x_{i} \partial x_{i}}\right) d U$

We note the following:

$\frac{\partial x_{j}^{\prime}}{\partial x_{i}}=\frac{\partial x_{j}}{\partial x_{i}}-\frac{\partial x_{c j}}{\partial x_{i}}=\frac{\partial x_{j}}{\partial x_{i}}=\delta_{j i}$ where $\delta_{j i}$ is the Kronecker delta, 


$$
\frac{\partial^{2} x_{j}^{\prime}}{\partial x_{i} \partial x_{i}}=\frac{\partial \delta_{j i}}{\partial x_{i}}=0
$$

and $n_{i} \delta_{j i}=n_{j}$. By making these substitutions into equation (R.5.2) we obtain:

$$
\int_{S_{o o}} G n_{j} d S=\int_{S_{o s}} \frac{\partial G}{\partial x_{i}} x_{j}^{\prime} n_{i} d S-\int_{u_{o o r}} x_{j}^{\prime} \frac{\partial^{2} G}{\partial x_{i} \partial x_{i}} d U
$$

Next, the discussion is limited to a scalar function $G=G(x, t)$ which satisfies the following two conditions:

a) $\quad G$ varies monotonously with the domain $u_{o \alpha}$, i.e. it attains no maximum or minimum in this domain. This is mathematically equivalent to:

$$
\nabla^{2} G=\frac{\partial^{2} G}{\partial x_{i} \partial x_{i}}=0
$$

b) Also, for $\mathrm{G}$ the following approximation is valid:

$$
\int_{S_{a a}} \frac{\partial G}{\partial x_{i}} x_{j}^{\prime} n_{i} d S \cong \frac{1}{S_{\alpha a}} \int_{\delta_{a u}} \frac{\partial G}{\partial x_{i}} d S \int_{S_{a \alpha}} x_{j}^{\prime} n_{i} d S \cong \frac{\partial \bar{G}}{\partial x_{i}} \int_{S_{u a}} x_{j}^{\prime} n_{i} d S
$$

where the assumption was made that the average of the gradient of the function being considered over the surface $\alpha-\alpha$ is approximately equal to the gradient of the average of the same function over the domain $u_{o \alpha}$.

Now we first expand (R.5.3) and then precede by substituting equations (R.5.4) and (R.5.5) into equation (R.5.3):

$$
\begin{aligned}
& \int_{S_{o u}} G n_{j} d S=\int_{S_{\alpha u}} \frac{\partial G}{\partial x_{i}} x^{\prime}{ }_{j} n_{i} d S+\int_{S_{\alpha \beta}} \frac{\partial G}{\partial x_{i}} x^{\prime}{ }_{j} n_{i} d S-\int_{u_{\alpha \alpha \alpha}} x^{\prime}, \frac{\partial^{2} G}{\partial x_{i} \partial x_{i}} d U \\
& \int_{S_{o \alpha}} G n_{j} d S=\frac{\partial G}{\partial x_{i}} \int_{S_{\alpha \alpha}} x^{\prime}{ }_{j} n_{i} d S+\int_{S_{\alpha \beta}} \frac{\partial G}{\partial x_{i}} x_{j}{ }_{j} n_{i} d S
\end{aligned}
$$

by applying Gauss' theorem to the L.H.S. of equation (R.5.6) we obtain:

$$
\int_{S_{o o}} G n_{j} d S=\int_{u_{o a}} \frac{\partial G}{\partial x_{j}} d U=U_{o u} \frac{\overline{\partial G}}{\partial x_{j}}
$$

next we replace the L.H.S. of equation (R.5.6) by its value from (R.5.7) and we obtain:

$$
U_{o x} \frac{\overline{\partial G}}{\partial x_{j}}=\frac{\partial \bar{G}}{\partial x_{i}} T_{i j}^{*}+\int_{S_{a \beta}} \frac{\partial G}{\partial x_{i}} x_{j}^{\prime} n_{i} d S
$$

where:

$$
T_{i j}^{*}=\int_{S_{a \alpha}} x_{j}^{\prime} n_{i} d S
$$

Bear and Bachmat [1990] pointed out that $T_{i j}^{*}$ represents the tortuosity of the phase within the REV. For convenience, we tabulated the five averaging rules in Table R.1. 
Table R.1. Summary of the averaging rules

\begin{tabular}{|c|c|}
\hline Type of average & Rule \\
\hline Sum (R.1) & $\overline{f(x, t)+g(x, t)}=\bar{f}+\bar{g}$ \\
\hline Product (R.2) & $\begin{array}{l}\overline{f g}=\overline{f g}+\overline{f^{\prime} g^{\prime}} \\
f^{\prime}: \text { the deviation of } f .\end{array}$ \\
\hline Time derivative (R.3) & $\begin{array}{l}\overline{\frac{\partial f}{\partial t}}=\frac{\partial \bar{f}}{\partial t}-\frac{1}{V_{R E V^{r}}} \int_{S} e u . n d S, \\
n: \text { outward unit normal vector, and } \\
u: \text { interphase velocity. }\end{array}$ \\
\hline Spatial derivative (R.4) & $\overline{\nabla f}=\nabla \bar{f}+\frac{1}{V_{R E V}} \int_{S} f n d S$ \\
\hline Spatial derivative satisfying $\nabla f^{2}=0($ R.5) & $\begin{array}{l}\overline{\frac{\partial f}{\partial x_{j}}}=\frac{\partial \bar{f}}{\partial x_{i}} T_{i j}^{*}+\frac{1}{V_{R E V}} \int_{S} x_{j}^{\prime} \frac{\partial f}{\partial x_{i}} n_{i} d S \\
T_{i j}^{*}=\frac{1}{V_{R E V}} \int_{S} n_{i} x_{j}^{\prime} d S, x^{\prime}=x-x^{c} \\
\text { where } x^{c} \text { is the coordinate of the centroid. }\end{array}$ \\
\hline
\end{tabular}

Next we apply these rules to obtain the macroscopic form of the generalized transport theorem.

\section{Appendix B.2 Mathematical Framework of the Macroscopic Balance Formulation}

\section{Mathematical Framework of Macroscopic Balance Formulation}

By integrating the general transport equation derived from microscopic consideration, over the larger domain $u_{o \alpha}$ of the REV and dividing by the volume of the REV ( $U_{o}$ ) we can begin the procedure for obtaining the macroscopic equations that are more meaning full in to the problem at hand.

$\frac{1}{U_{o}} \int_{u_{o u}} \frac{\partial e}{\partial t} d U=-\frac{1}{U_{o}} \int_{u_{o \alpha}} \nabla \cdot\left(e V+J^{E}\right) U U+\frac{1}{U_{o}} \int_{u_{o \alpha}} \rho \Gamma^{E} d U$

which can be rewritten in the following form:

$\frac{\overline{\partial e}}{\partial t}=-\overline{\nabla \cdot\left(e V+J^{E}\right)+\overline{\rho \Gamma^{E}}}$ 
where (..) refers to the volume average. For a two phase system, fluid and solid for our purpose, (A1.2) can be written for the fluid phase as follows:

$\left.\phi \frac{\overline{\partial e}}{\partial t}=-\phi \overline{\nabla \cdot\left(e V+J^{E}\right.}\right)+\phi \overline{\rho \Gamma^{E}}$

By applying rules (R.3) and (R.4) to equation (A1.3) we obtain:

$\left.\frac{\partial \phi \bar{e}}{\partial t}-\frac{1}{U_{o}} \int_{S_{\alpha \beta}} e u \cdot n d S=-\phi \nabla \cdot \overline{\left(e V+J^{E}\right.}\right)-\frac{1}{U_{o}} \int_{S_{\alpha \beta}}\left(e V+J^{E}\right) n d S+\phi \overline{\rho \Gamma^{E}}$

We can rearrange (A1.4) to obtain:

$\left.\frac{\partial \phi \bar{e}}{\partial t}=-\nabla \cdot \phi \overline{\left(e V+J^{E}\right.}\right)-\frac{1}{U_{o}} \int_{S_{\alpha \beta}} e(V-u) n d S-\frac{1}{U_{o}} \int_{S_{\alpha \beta}} J^{E} \cdot n d S+\phi \overline{\rho \Gamma^{E}}$

Here we describe each of the quantities in equation (A1.5):

- $\frac{\partial \phi \bar{e}}{\partial t}$ : Rate of increase of the extensive property $E$ per unit volume of porous media.

- $\phi \nabla \cdot\left(\overline{e V+J^{k}}\right)$ : Net influx of $E$ per unit volume due to advection and diffusion.

- $\frac{1}{U_{o}} \int_{S_{\alpha \beta}} e(V-u) n d S$ : Amount of $E$ entering the REV due to advection through the interface between the solid and the fluid per unit time.

- $\frac{1}{U_{o}} \int_{S_{\alpha \beta}} J^{E} . n d S$ : Amount of $E$ entering the REV due to diffusion through the interface between the solid and the fluid per unit time.

- $\phi \overline{\rho \Gamma^{E}}$ : Amount of E generated in the REV per unit time.

Next, by applying rules (R.1) and (R.2) to equation (A1.5). we obtain:

$\frac{\partial \phi \bar{e}}{\partial t}=-\nabla \cdot \phi\left(\bar{e} \bar{V}+\overline{e^{\prime} V^{\prime}}+\overline{J^{E}}\right)-\frac{1}{U_{o}} \int_{S_{\alpha \beta}} e(V-u) n d S-\frac{1}{U_{o}} \int_{S_{\alpha \beta}} J^{E} \cdot n d S+\phi \overline{\rho \Gamma^{E}}$

we note in this equation that the two integral terms need to be expressed in terms of readily measurable properties of the rock and fluid by making appropriate analysis and assumptions. Such development will be considered in the development of the momentum and energy balance equations.

\section{A2. Mass balance equation}

Let's start with the fluid case. By letting $E=m$ and $e=\rho$ and substituting in equation (A1.6) we obtain:

$\frac{\partial \phi \bar{\rho}}{\partial t}=-\nabla \cdot \phi\left(\bar{\rho} \bar{V}+\overline{\rho^{\prime} V^{\prime}}+\overline{J^{m}}\right)-\frac{1}{U_{o}} \int_{S_{\alpha \beta}} \rho(V-u) n d S-\frac{1}{U_{o}} \int_{S_{\alpha \beta}} J^{E} \cdot n d S+\phi \overline{\rho \Gamma^{E}}$

where the mass creation term is nonexistent because mass is nether generated nor created. If we assume the following:

i) The diffusive flux is much smaller than the advective mass flux that is: 


$$
\left|J^{m}\right| \ll|\rho V|
$$

ii) and that the solid liquid interface is impervious and there is no mass diffusion across the interphase, i.e.:

$$
\begin{aligned}
& \frac{1}{U_{o}} \int_{S_{\mathrm{\alpha} \beta}} \rho(V-u) n d S=0 \\
& \frac{1}{U_{o}} \int_{S_{u \beta}} J^{E} \cdot n d S=0
\end{aligned}
$$

then equation (A2.1) becomes:

$$
\frac{\partial \phi \bar{\rho}^{f}}{\partial t}=-\nabla \cdot \phi \bar{\rho}^{f} \bar{V}^{f}
$$

where the subscript $f$ indicates the fluid phase. Similarly we can write the mass balance equation for the solid phase as follows:

$$
\frac{\partial(1-\phi) \bar{\rho}^{s}}{\partial t}=-\nabla \cdot(1-\phi) \bar{\rho}^{s} \bar{V}^{s}
$$

\section{A3. Balance of linear momentum}

For this case, $E=M=m V^{m}, \Gamma^{M}=F$ (the intensity of the external body force acting on the phase), $e=\rho V^{m}$ and the diffusive flux is defined as $J^{M}=-\sigma$, where $\sigma$ is the stress and $V^{m}$ is defined as:

$V^{m}=\frac{1}{m} \sum_{\gamma=1}^{N} m^{\gamma} V^{\gamma}$

where the superscript $\gamma$ denotes a component within the phase. From equation (A3.1) we note that if the phase consists of a single component then the mass averaged velocity is simply equal to the velocity of the continuum phase, $V$.

We now substitute these quantities into the volume averaged generalized transport equation, (A1.6) we obtained the governing equation for momentum balance:

$$
\begin{aligned}
& \frac{\partial \phi \overline{\rho V^{m}}}{\partial t}=-\nabla \cdot \phi\left(\overline{\rho V^{m}} \bar{Y}^{m}+\overline{\rho V^{m} V^{m}}-\bar{\sigma}\right)-\frac{1}{U_{o}} \int_{S_{\alpha \beta}} \rho V^{m}\left(V^{m}-u\right) n d S \\
& +\frac{1}{U_{o}} \int_{S_{u \beta}} \sigma \cdot n d S+\phi \overline{\rho F}
\end{aligned}
$$

by applying rule (R.2) to the quantity $\overrightarrow{\rho \vec{V}^{m}}$ we get:

$$
\overline{\rho V^{m}}=\left(\bar{\rho} \overline{Y^{m}}+\overline{\rho^{\prime} V^{m}}\right.
$$

and by assuming that:

- the solid-fluid interphase is impervious (i.e the flow is in a tangential direction to the solid-liquid boundary), expressed mathematically by: 
$\frac{1}{U_{o}} \int_{S_{\alpha \beta}} \rho V^{m}\left(V^{m}-u\right) n d S=0$

- The dispersive fluxes are much smaller than the advective fluxes, i.e., :

$$
\overline{\rho^{\prime} V^{m}} \ll\left|\overline{\rho V^{m}}\right| \approx \bar{\rho} \overline{V^{m}}
$$

and:

$$
\left.\left|\widehat{\left(\rho V^{m}\right) V^{m} \mid}\right| \ll \mid \overline{\left(\rho V^{m}\right.}\right)^{m} \mid
$$

then equation (A3.2) reduces to the momentum balance equation for the fluid:

$$
\frac{\partial \phi \overline{\rho_{f}}\left(\overline{V_{f}}\right)}{\partial t}=-\nabla \cdot \phi\left(\bar{\rho}_{f} \bar{V}_{f} \bar{V}_{f}\right)+\frac{1}{U_{o}} \int_{S_{\alpha \beta}} \sigma_{f} \cdot n d S+\nabla \cdot \phi \bar{\sigma}_{f}+\overline{\phi \rho_{f} F_{f}}
$$

Similarly the momentum balance equation for the solid phase is:

$$
\begin{aligned}
& \frac{\partial(1-\phi) \overline{\rho_{s}}\left(\bar{V}_{s}\right)}{\partial t}=-\nabla \cdot(1-\phi)\left(\hat{\rho}_{s} \bar{V}_{s} \bar{V}_{s}\right)+\frac{1}{U_{o}} \int_{S_{\alpha \beta}} \sigma_{s} \cdot n d S+\nabla \cdot(1-\phi) \bar{\sigma}_{s} \\
& +(1-\phi) \overline{\rho_{s} F_{s}}
\end{aligned}
$$

we will next analyze the fluxes in the momentum balance equations.

A4. Analysis of the flux terms in the macroscopic momentum balance equation of the fluid We assume that the fluid is a newtonian fluid for which the stress $\sigma_{f}$ is made up of the viscous stress $\tau_{f}$ due to molecular interaction and of the fluid pressure $p$. Therefore, we can write:

$\sigma_{f}=\tau_{f}-p I$

where $I$ is the unit tensor.

Also we note that the body force caused by gravity is given by:

$F_{f}=-g \nabla Z$

where the $\mathrm{z}$-axis is directed upwards. First, we recombine the stress terms in equation (A3.5) in order to implement the correct averaging, i.e.:

$$
\frac{\partial \phi \overline{\rho_{f}}\left(\overline{V_{f}}\right)}{\partial t}=-\nabla \cdot \phi\left(\hat{\rho}_{f} \bar{V}_{f} \bar{V}_{f}\right)+\overline{\nabla \cdot \phi \sigma_{f}}+\phi \overline{\rho_{f} F_{f}}
$$

Next we substitute (A4.1) and (A4.2) into (A4.3) and apply averaging rule (R.1) to the stress term:

$\frac{\partial \phi \overline{\rho_{f}}\left(\overline{V_{f}}\right)}{\partial t}=-\nabla \cdot \phi\left(\bar{\rho}_{f} \bar{V}_{f} \bar{V}_{f}\right)+\overline{\nabla \cdot \phi \tau_{f}}-\overline{\phi \nabla p}-\phi \overline{\rho_{f}} g \nabla Z$

which can be written in the indicial notation as:

$$
\frac{\partial \phi \overline{\rho^{f}}\left(\overline{V_{i}^{f}}\right)}{\partial t}=-\frac{\partial}{\partial x_{j}}\left(\phi \bar{\rho}^{f} \bar{V}_{i}^{f} \bar{V}_{j}^{f}\right)+\phi \frac{\overline{\partial \tau_{i j}^{f}}}{\partial x_{j}}-\phi \frac{\overline{\partial p}}{\partial x_{i}}-\phi \bar{\rho} g \frac{\partial z}{\partial x_{i}}
$$

which can further be simplified as follows: 
$\phi \overline{\rho^{f}} \frac{\partial \overline{V_{i}^{f}}}{\partial t}+\overline{V_{i}^{f}} \frac{\partial \phi \overline{\rho^{f}}}{\partial t}=-\bar{V}_{i}^{f} \frac{\partial}{\partial x_{j}}\left(\phi \bar{\rho}^{f} \bar{V}_{j}^{f}\right)_{\phi} \bar{\rho}^{f} \bar{V}_{j}^{f} \frac{\partial}{\partial x_{j}}\left(\bar{V}_{i}^{f}\right.$,

$+\phi \frac{\overline{\partial \tau_{i j}^{f}}}{\partial x_{j}}-\phi \frac{\overline{\partial p}}{\partial x_{i}}-\phi \bar{\rho}^{f} g \frac{\partial z}{\partial x_{i}}$

Which can be written as:

$\phi \overline{\rho^{f}} \frac{\partial \overline{V_{i}^{f}}}{\partial t}+\overline{V_{i}^{f}}\left[\frac{\partial \phi \overline{\rho^{f}}}{\partial t}+\frac{\partial}{\partial x_{j}}\left(\phi \bar{\rho}^{f} \bar{V}_{j}^{f}\right)=-\phi \bar{\rho}^{f} \bar{V}_{j}^{f} \frac{\partial}{\partial x_{j}}\left(\bar{V}_{i}^{f}\right.\right.$,

$+\phi \frac{\overline{\partial \tau_{i j}^{f}}}{\partial x_{j}}-\phi \frac{\overline{\partial p}}{\partial x_{i}}-\phi \bar{\rho}^{f} g \frac{\partial z}{\partial x_{i}}$

We note that the term in the curly brackets equal zero (the continuity equation), therefore, we can write (A4.9) as:

$\phi \overline{\rho^{f}} \frac{\partial \overline{V_{i}^{f}}}{\partial t}=-\phi \bar{\rho}^{f} \bar{V}_{j}^{f} \frac{\partial \bar{V}_{i}^{f}}{\partial x_{j}}+\phi \frac{\overline{\partial \tau_{i j}^{f}}}{\partial x_{j}}-\phi \frac{\overline{\partial p}}{\partial x_{i}}-\phi \bar{\rho} f g \frac{\partial z}{\partial x_{i}}$

The pressure and inertial terms:

Next we analyze the terms in the R.H.S. of equation (A4.5) starting with the pressure term. We assume that the pressure varies monotonously in the REV, which is expressed mathematically by:

$\nabla p^{2} \equiv \frac{\partial^{2} p}{\partial x_{i} \partial x_{i}}=0$

and we precede by applying averaging rule (R.5):

$\overline{\frac{\partial p}{\partial x_{i}}}=\frac{\partial \bar{p}}{\partial x_{j}} T^{*}{ }_{j i}+\frac{1}{\phi U_{o}} \int_{S_{j s}} x_{i}^{\prime} \frac{\partial p}{\partial x_{j}} n_{j} d S$

Where:

$T^{*}{ }_{j i}=\frac{1}{U_{o}} \int_{S_{I f}} x_{i}^{\prime} n_{j} d S$

Now, in order to evaluate the surface integral in (A4.12a) Bear and Bachmat [1990] made the assumption that at the microscopic scale, the inertia and drag forces normal to the solid-fluid interface are negligible compared to the pressure forces and the gravity force, i.e.:

$\left|\left\{\left(\rho^{f} \frac{\partial V_{j}^{f}}{\partial t}+\rho^{f} V_{i}^{f} \frac{\partial V_{j}^{f}}{\partial x_{i}}\right)-\frac{\partial \tau_{i j}}{\partial x_{i}}\right] n_{j}\right| \ll<\left|\left(\frac{\partial p}{\partial x_{j}}+\rho^{f} g \frac{\partial z}{\partial x_{j}}\right) n_{j}\right|$

However, Levy et al. [1995] pointed out that the spatial term in the inertia expression $\left(\rho^{f} V_{i}^{f} \frac{\partial V_{j}^{f}}{\partial x_{i}}\right.$ ) can not be ignored especially when transients are present. This is due the fact that the paths of the velocity vectors change with distance due to the curvature in the porous medium. They suggested therefore to assume that: 


$$
\left\{\rho^{f} \frac{\partial V_{j}^{f}}{\partial t}-\frac{\partial \tau_{i j}}{\partial x_{i}}\right\} n_{j}|\ll|\left(\frac{\partial p}{\partial x_{j}}+\rho^{f} g \frac{\partial z}{\partial x_{j}}+\rho^{f} V_{i}^{f} \frac{\partial V_{j}^{f}}{\partial x_{i}}\right) n_{j} \mid
$$

We note that the change in the fluid shear stress can be considered small compared to the R.H.S. in equation (A4.14) because this stress can be assumed to be almost parallel to the solid surface.

Next we continue by dotting equation (A4.10) (written at the microscopic scale) with the normal unit vector outward from the solid-fluid surface pointing into the fluid, we obtain:

$$
\phi\left(\rho^{f} \frac{\partial V_{j}^{f}}{\partial t}-\frac{\partial \tau_{i j}^{f}}{\partial x_{i}}\right) n_{j}-\left(-\phi \rho^{f} V_{i}^{f} \frac{\partial V_{j}^{f}}{\partial x_{i}}-\phi \frac{\partial p}{\partial x_{j}}-\phi \rho^{f} g \frac{\partial z}{\partial x_{j}}\right) n_{j}=0
$$

by applying (A4.14) to (A4.15) we obtain:

$$
-\rho^{f} V_{i}^{f} \frac{\partial V_{j}^{f}}{\partial x_{i}} n_{j}-\frac{\partial p}{\partial x_{j}} n_{j}-\rho^{f} g \frac{\partial z}{\partial x_{j}} n_{j}=0
$$

We now rewrite the gravity force term as:

$$
\rho^{f} g \frac{\partial z}{\partial x_{j}}=\rho^{f} g \delta_{3 i}
$$

because when $x_{i}=z$ then $\frac{\partial z}{\partial x_{i}}=1=\delta_{33}$ otherwise $\frac{\partial z}{\partial x_{i}}=\delta_{31}=0$ or $\frac{\partial z}{\partial x_{i}}=\delta_{32}=0$.

Therefore we can rewrite equation (A4.16) as:

$$
-\rho^{\prime} V_{i}^{f} \frac{\partial V_{j}^{f}}{\partial x_{i}} n_{j}-\frac{\partial p}{\partial x_{j}} n_{j}-\rho^{f} g \delta_{3 i} n_{j}=0
$$

Next we assume that the deviation in density is negligible, i.e.:

$\rho^{f}=\bar{\rho}^{f}+\rho^{f} \cong \bar{\rho}^{f}$

With these assumptions we can precede to evaluate the surface integral in (A4.12a). By multiplying equation (A4.18) by $x^{\prime}$ and then integrating over the solid-liquid surface and then dividing through by $\phi U_{o}$ we obtain:

$$
-\frac{1}{\phi U_{o}} \int_{S_{\beta}} \rho^{f} V_{i}^{j} \frac{\partial V_{j}^{f}}{\partial x_{j}} x_{i}^{\prime} n_{j} d S-\frac{1}{\phi U_{o}} \int_{S_{j k}} \frac{\partial p}{\partial x_{j}} x_{i}^{\prime} n_{j} d S-\bar{\rho}^{j} g \delta_{3 i} \frac{1}{\phi U_{o}} \int_{S_{j}} x_{i}^{\prime} n_{j} d S=0
$$

and we note that:

$$
\begin{aligned}
& -\bar{\rho}^{f} g \delta_{3 i} \frac{1}{\phi U_{o}} \int_{S_{j S}} x_{i}^{\prime} n_{j} d S=-\bar{\rho}^{f} g \delta_{3 i}\left[\int_{S_{o f}} x_{i}^{\prime} n_{j} d S-\int_{S_{f l}} x_{i}^{\prime} n_{j} d S\right] \\
& =-\bar{\rho}^{f} g \delta_{3 i}\left[\delta_{i j}-T_{j i}^{*}\right]
\end{aligned}
$$


in obtaining the result in (A4.21) we used the definition (A4.12b) and also used Gauss' theorem as follows:

$$
\int_{S_{\text {of }}} x_{i}^{\prime} n_{j} d S=\int_{u_{o f}} \frac{\partial x_{i}^{\prime}}{\partial x_{j}} d U=\delta_{i j}
$$

Next we substitute (A4.21) into (A4.20) and rearrange to obtain:

$$
\frac{1}{\phi U_{o}} \int_{S_{j}} \frac{\partial p}{\partial x_{j}} x_{i}^{\prime} n_{j} d S=-\frac{1}{\phi U_{o}} \int_{S_{j s}} \rho^{f} V_{i}^{f} \frac{\partial V_{j}^{f}}{\partial x_{i}} x_{i}^{\prime} n_{j} d S-\bar{\rho}^{f} g\left(\delta_{3 i}-T_{3 i}^{*}\right)
$$

by combining (A4.12a) and (A4.22) we obtain:

$$
\frac{\overline{\partial p}}{\partial x_{i}}=\frac{\partial \bar{p}}{\partial x_{j}} T^{*}{ }_{j i}-\frac{1}{\phi U_{0}} \int_{S_{f i}} \rho^{f} V_{i}^{f} \frac{\partial V_{j}^{f}}{\partial x_{i}} x_{i}^{\prime} n_{j} d S-\bar{\rho}^{f} g\left(\delta_{3 i}-T_{3 i}^{*}\right)
$$

Now we substitute (A4.17) and (A4.23) into (A4.10):

$\phi \overline{\rho^{f}} \frac{\partial \overline{V_{i}^{f}}}{\partial t}=-\phi \bar{\rho} f \bar{V}_{j}^{f} \frac{\partial \bar{V}_{i}^{f}}{\partial x_{j}}+\phi \frac{\overline{\partial \tau_{i j}^{f}}}{\partial x_{j}}-\phi \frac{\partial \bar{p}}{\partial x_{j}} T^{*}{ }_{j i}+\frac{1}{U_{o}} \int_{S_{\beta}} \rho^{f} V_{j}^{f} \frac{\partial V_{i}^{f}}{\partial x_{j}} x_{i}^{\prime} n_{j} d S+$

$\phi \bar{\rho}^{f} g\left(\delta_{3 i}-T_{3 i}^{*}\right)-\phi \bar{\rho}^{f} g \delta_{3 i}$

which is equal to:

$\phi \overline{\rho^{f}} \frac{\partial \overline{V_{i}^{f}}}{\partial t}=-\phi \bar{\rho} f \bar{V}_{j}^{f} \frac{\partial \bar{V}_{i}^{f}}{\partial x_{j}}+\phi \frac{\overline{\partial \tau_{i j}^{f}}}{\partial x_{j}}-\phi\left(\frac{\partial \bar{p}}{\partial x_{j}}+\bar{\rho}^{f} g \frac{\partial z}{\partial x_{j}}\right) T_{j l}^{*}+$

$\frac{1}{U_{o}} \int_{S_{j}:} \rho^{f} V_{i}^{f} \frac{\partial V_{j}^{f}}{\partial x_{i}} x_{i}^{\prime} n_{j} d S$

Also another useful equation is obtained by substituting (A4.17) and (A4.23) into (A4.5) to obtain:

$$
\begin{aligned}
& \frac{\partial \phi \overline{\rho^{f}}\left(\overline{Y_{i}^{f}}\right)}{\partial t}=-\frac{\partial}{\partial x_{j}}\left(\phi \bar{\rho}^{f} \bar{V}_{i}^{f} \bar{V}_{j}^{f}\right)+\phi \frac{\overline{\partial \tau_{i j}^{f}}}{\partial x_{j}}-\phi\left(\frac{\partial \bar{p}}{\partial x_{j}}+\bar{\rho}^{f} g \frac{\partial z}{\partial x_{j}}\right) T^{*}{ }_{j i} \\
& +\frac{1}{U_{o}} \int_{S_{\beta}} \rho^{f} V_{i}^{f} \frac{\partial V_{j}^{f}}{\partial x_{i}} x_{i}^{\prime} n_{j} d S
\end{aligned}
$$

Levy et al., [1995] proposed a that the surface integral in (A3.25) is a quadratic Forchheimer term that is caused by the solid-fluid drag. They proposed the following form:

$-\sqrt{\frac{c_{v} \alpha_{m}}{\Delta_{f}^{2}}} \phi \rho^{f}\left|V_{f}-V_{s}\right|\left(V_{f_{i}}-V_{s_{i}}\right)$

where $c_{v}$ is a shape factor, $\alpha_{m}$ is a characteristic parameter of the configuration of the surface $S_{f s}$, and $\Delta_{f}$ is the hydraulic radius defined as:

$$
\Delta_{f}=\frac{U_{o f}}{S_{f s}}
$$


Other authors ([Hsu and Cheng, 1990] and [Nield, 1991]) suggested similar forms of the Forchheimer term but Levy's formula is more general in that the solid may or may not be at rest. By substituting equation (A4.27) into (A4.26) we obtain:

$$
\begin{aligned}
& \frac{\partial \phi \overline{\rho^{f}}\left(\overline{V_{i}^{f}}\right)}{\partial t}=-\frac{\partial}{\partial x_{j}}\left(\phi \bar{\rho}^{f} \bar{V}_{i}^{f} \bar{V}_{j}^{f}\right)+\phi \frac{\overline{\partial \tau_{i j}^{f}}}{\partial x_{j}}-\phi\left(\frac{\partial \bar{p}}{\partial x_{j}}+\bar{\rho}^{f} g \frac{\partial z}{\partial x_{j}}\right) T_{j i}^{*} \\
& -\sqrt{\frac{c_{v} \alpha_{m}}{\Delta_{f}^{2}}} \phi \rho^{f} \mid V_{f}-V_{s}\left(V_{f_{i}}-V_{s_{i}}\right)
\end{aligned}
$$

where the minus sign in front of the Forchheimer term is there because this force is opposing to the fluid flow at the pore wall.

\section{The viscous drag term}

For our purpose we consider a compressible and isotropic Newtonian fluid for which the constitutive relationship relating viscous drag to the rate of change of velocity is given at the microscopic scale by:

$$
\tau_{i j}=\mu\left(\frac{\partial V_{i}}{\partial x_{j}}+\frac{\partial V_{j}}{\partial x_{i}}\right)+\lambda\left(\frac{\partial V_{1}}{\partial x_{1}}+\frac{\partial V_{2}}{\partial x_{2}}+\frac{\partial V_{3}}{\partial x_{3}}\right)
$$

Where $\mu$ is the dynamic viscosity of and $\lambda$ is another viscosity coefficient of the liquid and the quantity $\left(\mu+\frac{2}{3} \lambda\right)$ is sometimes referred to as the bulk (or dilalational) viscosity.

We proceed by taking the spatial derivative of (A4.1) to obtain:

$$
\frac{\tau_{i j}}{\partial x_{j}}=\frac{\partial \mu}{\partial x_{j}}\left(\frac{\partial V_{i}}{\partial x_{j}}+\frac{\partial V_{j}}{\partial x_{i}}\right)+\frac{\partial \lambda}{\partial x_{i}}\left(\frac{\partial V_{j}}{\partial x_{j}}\right)+\mu \frac{\partial^{2} V_{i}}{\partial x_{j} \partial x_{j}}+(\mu+\lambda) \frac{\partial}{\partial x_{i}}\left(\frac{\partial V_{j}}{\partial x_{j}}\right)
$$

We note that the variation with distance of both the bulk and dynamic viscosities are negligible. Therefore, equation (A4.30) reduces to:

$$
\frac{\tau_{i j}}{\partial x_{j}}=\mu \frac{\partial^{2} V_{i}}{\partial x_{j} \partial x_{j}}+(\mu+\lambda) \frac{\partial}{\partial x_{i}}\left(\frac{\partial V_{j}}{\partial x_{j}}\right)
$$

Now we average equation (A4.31). First we used averaging rule (R.2, average of a product):

$$
\overline{\frac{\tau_{i j}}{\partial x_{j}}}=\bar{\mu} \frac{\partial^{2} V_{i}}{\partial x_{j} \partial x_{j}}+\overline{\mu^{\prime}\left(\frac{\partial^{2} V_{l}}{\partial x_{j} \partial x_{j}}\right)}+\overline{(\mu+\lambda)} \overline{\frac{\partial}{\partial x_{i}}\left(\frac{\partial V_{j}}{\partial x_{j}}\right)}+\overline{(\mu+\lambda) \frac{\partial}{\partial x_{j}}\left(\frac{\partial V_{j}}{\partial x_{j}}\right)}
$$

and we assume that

$$
\left|\bar{\mu} \overline{\frac{\partial^{2} V_{i}}{\partial x_{j} \partial x_{j}}}\right|>\left|\overline{\mu^{\prime}\left(\frac{\partial^{2} V_{i}}{\partial x_{j} \partial x_{j}}\right)}\right|
$$


and

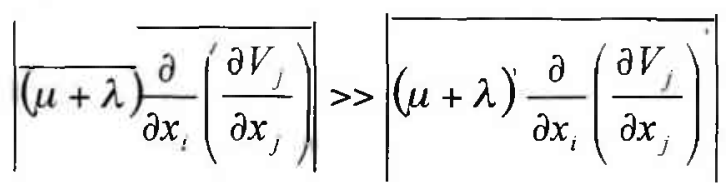

to obtain:

$$
\overline{\frac{\boldsymbol{\tau}_{i j}}{\partial x_{j}}}=\bar{\mu} \overline{\frac{\partial^{2} V_{i}}{\partial x_{j} \partial x_{j}}}+\overline{(\mu+\lambda) \overline{\frac{\partial}{\partial x_{i}}\left(\frac{\partial V_{j}}{\partial x_{j}}\right)}}
$$

Which can be further simplified using averaging rule (R.1, average of a sum):

$$
\phi \frac{\overline{\tau_{i j}}}{\partial x_{j}}=\bar{\mu} \phi \overline{\frac{\partial^{2} V_{i}}{\partial x_{j} \partial x_{j}}}+(\bar{\mu}+\bar{\lambda}) \overline{\frac{\partial}{\partial x_{i}}\left(\overline{\frac{\partial V_{j}}{\partial x_{j}}}\right)}
$$

Were we have multiplied across by the porosity.

Next we recall that the averaging rule for a spatial derivative written in indicial notation is given by:

$$
\phi \frac{\overline{\partial G_{i}}}{\partial x_{j}}=\frac{\partial\left(b \overline{G_{i}}\right)}{\partial x_{j}}+\frac{1}{U_{o}} \int_{S_{\beta}} G_{i} n_{j} d S
$$

Let $G_{i}=\frac{\partial V_{i}}{\partial x_{j}}$ and substitute this value in equation (R.4) to obtain:

$\phi \overline{\frac{\partial^{2} V_{i}}{\partial x_{j} \partial x_{j}}}=\frac{\partial}{\partial x_{j}}\left(\phi \frac{\overline{\partial V_{i}}}{\partial x_{j}}\right)+\frac{1}{U_{o}} \int_{S_{f}} \frac{\partial V_{i}}{\partial x_{j}} n_{j} d S$

And by applying (R.4) to $\phi \frac{\overline{\partial V_{i}}}{\partial x_{j}}$ in (A4.37) we obtain:

$\phi \overline{\frac{\partial^{2} V_{i}}{\partial x_{j} \partial x_{j}}}=\frac{\partial}{\partial x_{j}}\left(\frac{\partial}{\partial x_{j}}\left[\phi \bar{V}_{i}\right]+\frac{1}{U_{o}} \int_{S_{S s}} V_{i} n_{j} d S\right)+\frac{1}{U_{o}} \int_{S_{S s}} \frac{\partial V_{i}}{\partial x_{j}} n_{j} d S$

This simplifies to:

$$
\phi \overline{\frac{\partial^{2} V_{i}}{\partial x_{j} \partial x_{j}}}=\frac{\partial^{2} \phi \bar{V}_{i}}{\partial x_{j} \partial x_{j}}+\frac{\partial}{\partial x_{j}}\left(\frac{1}{U_{o}} \int_{S_{f s}} V_{i} n_{j} d S\right)+\frac{1}{U_{o}} \int_{S_{f s}} \frac{\partial V_{i}}{\partial x_{j}} n_{j} d S
$$

Similarly:

$$
\overline{\frac{\partial}{\partial x_{i}}\left(\frac{\partial V_{j}}{\partial x_{j}}\right)}=\frac{\partial^{2} \phi \bar{V}_{j}}{\partial x_{i} \partial x_{j}}+\frac{\partial}{\partial x_{i}}\left(\frac{1}{U_{o}} \int_{S_{s s}} V_{j} n_{j} d S\right)+\frac{1}{U_{o}} \int_{S_{j s}} \frac{\partial V_{j}}{\partial x_{j}} n_{i} d S
$$

By substituting (A4.39) and (A4.40) into (A4.36) we obtain: 


$$
\begin{aligned}
& \phi \frac{\overline{\tau_{i j}}}{\partial x_{j}}=\bar{\mu}\left\{\frac{\partial^{2} \phi \bar{V}_{i}}{\partial x_{j} \partial x_{j}}+\frac{\partial}{\partial x_{j}}\left(\frac{1}{U_{o}} \int_{S_{j s}} V_{i} n_{j} d S\right)+\frac{1}{U_{o}} \int_{S_{j}} \frac{\partial V_{i}}{\partial x_{j}} n_{j} d S\right\} \\
& +(\bar{\mu}+\bar{\lambda})\left\{\frac{\partial^{2} \phi \overline{V_{j}}}{\partial x_{i} \partial x_{j}}+\frac{\partial}{\partial x_{i}}\left(\frac{1}{U_{o}} \int_{S_{j s}} V_{j} n_{j} d S\right)+\frac{1}{U_{o}} \int_{S_{j s}} \frac{\partial V_{j}}{\partial x_{j}} n_{i} d S\right\}
\end{aligned}
$$

Next we evaluate the surface integrals in equation (A4.41). We first assume a no-slip condition at the solid-liquid inter-phase, i.e.:

$$
V_{f}\left|S_{f s}=V_{s}\right| S_{f s}
$$

We can start to evaluate the term $\frac{1}{U_{o}} \int_{S_{j s}} V_{i} n_{j} d S$. We first note that since the solid is relatively rigid, that we can assume that the average solid velocity to be approximately equal to the intrinsic volume averaged velocity of the solid, that is:

$$
V_{s i}=\bar{V}_{s i}
$$

With this we can write the surface integral being evaluated as:

$$
\frac{1}{U_{o}} \int_{S_{k}} V_{i} n_{j} d S=\frac{1}{U_{o}} \int_{S_{j s}} V_{s i} n_{j} d S=\frac{1}{U_{o}} \int_{S_{j s}} \bar{V}_{s i} n_{j} d S=\bar{V}_{s i} \frac{1}{U_{o}} \int_{S_{j s}} n_{j} d S
$$

To evaluate the last surface integral in (A4.44) we recall the averaging rule for a spatial derivative:

$$
\phi \frac{\overline{\partial G_{i}}}{\partial x_{j}}=\frac{\partial}{\partial x_{j}}\left(\phi \overline{G_{i}}\right)+\int_{S_{f_{A}}} G_{i} n_{j} d S
$$

And we let $G_{i}=1$ therefore:

$$
\overline{G_{i}}=\frac{1}{U_{\text {of }}} \int_{u_{o f}} G_{i} d U=\frac{U_{\text {of }}}{U_{\text {of }}}=1
$$

By substituting these values in (R.4) and noting that $\frac{\partial G_{i}}{\partial x_{j}}=0$ we obtain:

$$
\int_{S_{\xi}} G_{i} n_{j} d S=-\frac{\partial \phi}{\partial x_{j}}
$$


By substituting (A4.45) into (A4.44) we obtain:

$\frac{1}{U_{o}} \int_{S_{s i}} V_{i} n_{j} d S=-\bar{V}_{s i} \frac{\partial \phi}{\partial x_{j}}=\phi \frac{\partial \bar{V}_{s i}}{\partial x_{j}}-\frac{\partial \phi \bar{V}_{s i}}{\partial x_{j}}$

Next we turn our attention to the term $\frac{1}{U_{o}} \int_{S_{s}} \frac{\partial V_{j}}{\partial x_{j}} n_{i} d S$. By assuming that the diffusive flux near the solid liquid inter-phase is negligible, we can write:

$\frac{1}{U_{o}} \int_{S_{j}} \frac{\partial V_{j}}{\partial x_{j}} n_{i} d S=\overline{\frac{\partial V_{j}}{\partial x_{j}}} \frac{1}{U_{o}} \int_{S_{j s}} n_{i} d S$

But as shown previously:

$$
\int_{S_{s s}} G_{i} n_{i} d S=-\frac{\partial \phi}{\partial x_{i}}
$$

Therefore:

$\frac{1}{U_{0}} \int_{S_{\mathrm{A}}} \frac{\partial V_{j}}{\partial x_{j}} n_{i} d S=-\overline{\frac{\partial V_{j}}{\partial x_{j}}} \frac{\partial \phi}{\partial x_{i}}$

We recall the general form of the transport equation of any extensive property at the macroscopic scale:

$$
\frac{\partial \phi \bar{e}}{\partial t}=-\nabla \cdot \phi\left(\bar{V}+\overline{e^{\prime} V^{\prime}}+\overline{J^{E}}\right)-\frac{1}{U_{o}} \int_{S_{\alpha \beta}} e(V-u) n d S-\frac{1}{U} \int_{S_{\alpha \beta}} J^{E} \cdot n d S+\phi \overline{\rho \Gamma^{E}}
$$

And let consider the volume balance, i.e. $e=1$, and we recall that equation (A1.6) can be written at the microscopic scale as:

$\frac{\partial e}{\partial t}=-\nabla \cdot(e V+J)+\rho \Gamma$

Where $J=V-V=0$. Therefore, by substituting $e=1$ into (1.1) we obtain:

$\rho \Gamma=\nabla \cdot V$

By letting $e=1$ and substituting $\rho \Gamma=\nabla \cdot V$ into (A1.6) we obtain: 
$\frac{\partial \phi}{\partial t}=-\nabla \cdot \phi \bar{V}-\phi \overline{\nabla \cdot V}$

Where in (A4.51) we have assumed that the surface between the solid and the fluid is material, that there is no flux across the surface, and that the advective deviation term is negligible. Now we write equation (A4.51) in indicial notation:

$\frac{\partial \phi}{\partial t}=-\frac{\partial \phi \bar{V}_{j}}{\partial x_{j}}-\phi \frac{\overline{\partial V_{j}}}{\partial x_{j}} \Rightarrow \frac{\overline{\partial V_{j}}}{\partial x_{j}}=-\frac{1}{\phi}\left(\frac{\partial \phi}{\partial t}+\frac{\partial \phi \bar{V}_{j}}{\partial x_{j}}\right)$

Finally we substitute (A4.52) into (A4.49) to obtain:

$\frac{1}{U_{o}} \int_{S_{j}} \frac{\partial V_{j}}{\partial x_{j}} n_{i} d S=\frac{\overline{\partial V_{j}}}{\partial x_{j}} \frac{\partial \phi}{\partial x_{i}}=-\frac{1}{\phi}\left(\frac{\partial \phi}{\partial t}+\frac{\partial \phi \bar{V}_{j}}{\partial x_{j}}\right) \frac{\partial \phi}{\partial x_{i}}$

We next evaluate the term $\frac{1}{U_{o}} \int_{S_{S s}} \frac{\partial V_{i}}{\partial x_{j}} n_{j} d S$. We focus our attention on the region near the

fluid-solid surface. We construct an orthogonal reference frame $\left(n, t^{\prime}, t^{\prime \prime}\right)$ where $n$ is the normal unit vector, $t^{\prime}$ and $t^{\prime \prime}$ are two mutually orthogonal unit tangent vectors. In this frame, we can represent the fluid velocity vector by:

$V=V_{j} n_{j} n+V_{j} t_{j}{ }^{\prime} t^{\prime}+V_{j} t_{j}{ }^{\prime \prime} t^{\prime \prime}$

Similarly, the solid velocity vector can be represented by:

$V_{s}=V_{s j} n_{j} n+V_{s j} t_{j}^{\prime} t^{\prime}+V_{s j} t_{j}^{\prime \prime} t^{\prime \prime}$

By subtracting (A4.55) from (A4.54) we obtain:

$\left(V-V_{s}\right)=\left(V_{j}-V_{s j}\right)\left(n_{j} n+t_{j}{ }^{\prime} t^{\prime}+t_{j}{ }^{\prime}{ }^{\prime \prime}\right)$

And by dotting (A4.56) with the unit vector $1 x_{i}$ we obtain:

$\left(V_{i}-V_{s i}\right)=\left(V_{j}-V_{s j}\right)\left(n_{j} n_{i}+t_{j}{ }^{\prime} t_{i}^{\prime}+t_{j}^{\prime \prime} t_{i}^{\prime \prime}\right)$

Also we note the identity:

$n_{j} n_{i}+t_{j}{ }^{\prime} t_{i}+t_{j}{ }^{\prime} t_{i}^{\prime \prime}=\delta_{i j}$ 
The velocity components of the solid and the fluid can also be evaluated in the reference frame $\left(t^{\prime}, t^{\prime \prime}\right)$ by:

$$
\left(V_{i}^{t}-V_{s i}^{t}\right)=\left(V_{j}-V_{s j}\right)\left(t_{j}{ }^{\prime} t^{\prime}{ }_{i}+t_{j}{ }^{\prime \prime} t_{i}{ }^{\prime \prime}\right)=\left(V_{j}-V_{s j}\right)\left(\delta_{i j}-n_{j} n_{i}\right)
$$

Where the $t$ superscript indicates that these velocity components are on the surfaces of the solid.

With (A4.59) we can evaluate $\frac{1}{U_{o}} \int_{S_{F}} \frac{\partial V_{i}}{\partial x_{j}} n_{j} d S$ as follows:

$$
\begin{aligned}
& \frac{1}{U_{o}} \int_{S_{f s}} \frac{\partial V_{i}}{\partial x_{j}} n_{j} d S \cong \frac{1}{U_{o}} \int_{S_{f s}} \frac{V_{s i}^{t}-V_{i} \mid \Delta}{\Delta} d S \cong \frac{1}{U_{o} \Delta_{c}} \int_{S_{j s}}\left(V_{s j}-V_{j}\right)\left(\delta_{i j}-n_{j} n_{i}\right) d S \\
& =\frac{S_{f s}}{\Delta_{c} U_{o}}\left\{\left(\bar{V}_{s j}-\bar{V}_{j}\right)\left(\delta_{i j}-\frac{1}{S_{f s}} \int_{S_{f s}} n_{j} n_{i} d S\right)\right\}
\end{aligned}
$$

Where the term $V_{i} \mid \Delta$ refers to the fluid velocity at an elementary distance from the surface and $\Delta_{c}$ is a characteristic distance that provides a measure of the pore size. One way to evaluate $\Delta_{c}$ is by proportionality to the hydraulic radius (defined as the ratio $\frac{U_{o f}}{S_{f s}}$ ) of the pore $\Delta_{f}$, i.e.:

$$
\Delta_{c}=\frac{\Delta_{f}}{C_{f}}
$$

Where $C_{f}$ is a dimensionless shape factor. With these new terms, equation (A4.32) can be modified to the form:

$$
\frac{1}{U_{o}} \int_{S_{f s}} \frac{\partial V_{i}}{\partial x_{j}} n_{j} d S=-\frac{\phi C_{f} \alpha_{i j}}{\Delta_{f}^{2}} \bar{V}_{r j}
$$

Where

$\alpha_{i j}=\delta_{i j}-\frac{1}{S_{f s}} \int_{S_{f s}} n_{j} n_{i} d S$

And

$$
\bar{V}_{r j}=\bar{V}_{j}-\bar{V}_{s j}
$$


By substituting (A4.46), (A4.49), (A4.53), and (A4.60) into (A4.41) we obtain:

$$
\begin{aligned}
& \phi \frac{\overline{\tau_{i j}}}{\partial x_{j}}=\bar{\mu}\left\{\frac{\partial^{2} \phi \overline{V_{r i}}}{\partial x_{j} \partial x_{j}}+\frac{\partial}{\partial x_{j}}\left(\phi \frac{\partial \bar{V}_{s i}}{\partial x_{j}}\right)\right\} \\
& +(\bar{\mu}+\bar{\lambda})\left[\frac{\partial^{2} \phi \bar{V}_{i j}}{\partial x_{i} \partial x_{j}}+\frac{\partial}{\partial x_{i}}\left(\phi \frac{\partial \bar{V}_{s j}}{\partial x_{j}}\right)-\frac{1}{\phi}\left(\frac{\partial \phi}{\partial t}+\frac{\partial \phi \bar{V}_{j}}{\partial x_{j}}\right) \frac{\partial \phi}{\partial x_{i}}\right\} \\
& -\frac{\phi C_{j} \alpha_{i j}}{\Delta_{f}^{2}} \bar{V}_{r j}
\end{aligned}
$$

Next we assume the medium is the medium is approximately homogeneous, i.e.: $\frac{\partial \phi}{\partial x_{j}} \approx 0$. With this assumption, equation (A4.63) reduces to:

$\phi \frac{\overline{\partial \tau_{i j}}}{\partial x_{j}}=\bar{\mu} \phi \frac{\partial^{2} \bar{V}_{i}}{\partial x_{j} \partial x_{j}}+(\bar{\mu}+\bar{\lambda}) \phi \frac{\partial^{2} \bar{V}_{j}}{\partial x_{i} \partial x_{j}}-\bar{\mu} \frac{\phi C_{f} \alpha_{i j}}{\Delta_{f}^{2}} \bar{V}_{r j}$

By substituting equation (A4.64) into (A4.28) we obtain the following final form of the momentum balance equation for the fluid:

$$
\begin{aligned}
& \frac{\partial \phi \overline{\rho^{f}}\left(\overline{V_{i}^{f}}\right)}{\partial t}=-\frac{\partial}{\partial x_{j}}\left(\phi \bar{\rho}^{f} \bar{V}_{i}^{f} \bar{V}_{j}^{f}\right)+\bar{\mu}^{f} \phi \frac{\partial^{2} \bar{V}_{i}^{f}}{\partial x_{j} \partial x_{j}}+\left(\bar{\mu}^{f}+\bar{\lambda}^{f}\right) \phi \frac{\partial^{2} \bar{V}_{j}^{f}}{\partial x_{i} \partial x_{j}}-\bar{\mu}^{f} \frac{\phi C_{f} \alpha_{i j}}{\Delta_{f}^{2}} \bar{V}_{r j} \\
& -\phi\left(\frac{\partial \bar{p}}{\partial x_{j}}+\bar{\rho}^{f} g \frac{\partial z}{\partial x_{j}}\right) T^{*}{ }_{j i}-\sqrt{\frac{c_{v} \alpha_{m}}{\Delta_{f}^{2}}} \phi \rho^{f}\left|V^{f}-V^{s}\right|\left(V_{i}^{f}-V_{i}^{s}\right)
\end{aligned}
$$

\section{A5. Analysis of the flux terms in the macroscopic momentum balance equation of the solid}

Trezaghi [1925] (also see [Bear and Bachmat, 1990]), considering a solid granular matrix where the grains are approximately surrounded by ambient fluid, proposed that on the macroscopic scale the strain causing stress (effective stress, $\sigma_{s}{ }^{\prime}$ ) in the solid is caused by the difference between the stress within the solids and a second stress caused by the stress in the fluid and equal to the later in magnitude, therefore:

$$
\bar{\sigma}_{s}^{\prime}=(1-\phi)\left(\bar{\sigma}_{s}-\bar{\sigma}_{f}\right)
$$

Let's use this concept to determine the stress in the solid phase. First, consider the momentum equation for the solid and the fluid: 


$$
\begin{aligned}
& \frac{\partial(1-\phi) \bar{\rho}^{s}\left(\bar{V}^{s}\right)}{\partial t}=-\nabla \cdot(1-\phi)\left(\bar{\rho}^{s} \bar{V}^{s} \bar{V}^{s}\right)+\frac{1}{U_{o}} \int_{S_{s}} \sigma_{s} \cdot n d S+\nabla \cdot(1-\phi) \bar{\sigma}+(1-\phi) \overline{\rho^{s} F^{s}} \\
& \frac{\partial \phi \bar{\rho}^{f}\left(\bar{V}^{f}\right)}{\partial t}=-\nabla \cdot \phi\left(\bar{\rho} f \bar{V}^{f} \bar{V}^{f}\right)+\frac{1}{U_{o}} \int_{S_{f s}} \sigma_{f} \cdot n d S+\nabla \cdot \phi \bar{\sigma}_{f}+\phi \overline{\rho^{f} F^{f}}
\end{aligned}
$$

We note that the momentum exchange terms (the surface integrals) in (A5.2a, and b) must be equal in magnitude and opposite in direction for equilibrium to occur. With this observation, we obtain the following momentum balance equation for the porous medium when we add equations (A5.1) and (A5.2):

$$
\begin{aligned}
& \frac{\partial \phi \bar{\rho}^{f}\left(\bar{V}^{f}\right)}{\partial t}+\frac{\partial(1-\phi) \bar{\rho}^{s}\left(\bar{V}^{s}\right)}{\partial t}=-\nabla \cdot \phi\left(\bar{\rho}^{f} \bar{V}^{f} \bar{V}^{f}\right)-\nabla \cdot(1-\phi)\left(\bar{\rho}^{s} \bar{V}^{s} \bar{V}^{s},\right. \\
& +\nabla \cdot \bar{\sigma}_{t}+\phi \overline{\rho^{f} F^{f}}+(1-\phi) \overline{\rho^{s} F^{s}}
\end{aligned}
$$

Where

$$
\bar{\sigma}_{\imath}=\phi \bar{\sigma}_{f}+(1-\phi) \bar{\sigma}_{s}=\bar{\sigma}_{s}{ }^{\prime}+\bar{\sigma}_{f}
$$

By substituting (A5.4) into (A5.3) we obtain:

$$
\begin{aligned}
& \left.\frac{\partial \phi \bar{\rho}^{f}\left(\bar{V}^{f}\right)}{\partial t}+\frac{\partial(1-\phi) \bar{\rho}^{s}\left(\bar{V}^{s}\right)}{\partial t}=-\nabla \cdot \phi\left(\bar{\rho}^{f} \bar{V}^{f} \bar{V}^{f}\right)-\nabla \cdot(1-\phi)\right)_{\rho}^{s} \bar{V}^{s} \bar{V}^{s}, \\
& +\nabla \cdot \bar{\sigma}_{s}{ }^{\prime}+\nabla \cdot \bar{\sigma}_{f}+\phi \overline{\rho^{f} F^{f}}+(1-\phi) \overline{\rho^{s} F^{s}}
\end{aligned}
$$

And by subtracting the fluid momentum balance equation from (A5.5) we obtain:

$$
\left.\left.\frac{\partial(1-\phi) \rho{ }^{s}\left(\bar{V}^{s}\right)}{\partial t}=-\nabla \cdot(1-\phi)\right) \cdot \bar{\rho}^{s} \bar{V} s \bar{V} s\right)+\nabla \cdot \bar{\sigma}_{s}{ }^{\prime}+\nabla \cdot(1-\phi) \bar{\sigma}_{f}-\frac{1}{U_{a}} \int_{S_{s}} \sigma_{f} \cdot n d S+(1-\phi) \overline{\rho^{s} F^{s}}
$$

Which can be written in indicial notation as?

$$
\begin{aligned}
& \frac{\partial(1-\phi) \bar{\rho}^{s}\left(\bar{V}_{i}^{s}\right)}{\partial t}=-\frac{\partial}{\partial x_{j}}\left[(1-\phi)\left(\epsilon^{s} \bar{V}_{i}^{s} \bar{V}_{j}^{s}\right)\right] \frac{\partial \bar{\sigma}_{s i j}{ }^{\prime}}{\partial x_{j}}+\frac{\partial(1-\phi) \bar{\sigma}_{f i j}}{\partial x_{j}}-\frac{1}{U_{o}} \int_{S_{j s}} \sigma_{f i} n_{j j} d S \\
& -(1-\phi))^{s} \frac{\partial z}{\partial x_{i}}
\end{aligned}
$$

Where we have substituted for the body force with the gravity acting on the solid. 
We recall that for a fluid the stress is given by:

$$
\sigma_{i j}=\tau_{i j}-p \delta_{i j}
$$

Therefore, we can write:

$$
\frac{\partial(1-\phi) \bar{\sigma}_{f i j}}{\partial x_{j}}=\frac{\partial(1-\phi)\left(\bar{\tau}_{i j}-p \delta_{i j}\right)}{\partial x_{j}}=\frac{\partial(1-\phi) \boldsymbol{t}_{i j}}{\partial x_{j}}-\frac{\partial(1-\phi) p}{\partial x_{i}} \cong-\frac{\partial(1-\phi) p}{\partial x_{i}}
$$

Where we have assumed in (A5.9) that the effect of the viscous resistance in the bulk fluid has a negligible effect within the solid.

Our next objective is to evaluate the surface integral term in (A5.7).

And we proceed to evaluate the integral as follows:

$-\frac{1}{U_{o}} \int_{S_{f s}} \sigma_{f i} . n_{f j} d S=-\frac{1}{U_{o}} \int_{S_{f s}}\left(\tau_{i j}-p \delta_{i j}\right) n_{f j} d S=-\frac{1}{U_{o}} \int_{S_{f s}} \tau_{f i} n_{f j} d S+\frac{1}{U_{o}} \int_{S_{f s}} p . n_{f i} d S$

Where the identity $n_{f i}=n_{f j} \delta_{i j}$ was used in the last surface integral in the R.H.S. of (A5.10). By using averaging rule (R.4) we obtain:

$\phi \frac{\overline{\partial \tau_{i j}}}{\partial x_{j}}=\frac{\partial \phi \overline{\tau_{i j}}}{\partial x_{j}}+\frac{1}{U_{o}} \int_{S_{f i}} \tau_{f i} \cdot n_{f j} d S$

Here we recall equation (A4.64):

$\phi \frac{\overline{\partial \tau_{i j}}}{\partial x_{j}}=\bar{\mu} \phi \frac{\partial^{2} \bar{V}_{i}}{\partial x_{j} \partial x_{j}}+(\bar{\mu}+\bar{\lambda}) \phi \frac{\partial^{2} \bar{V}_{j}}{\partial x_{i} \partial x_{j}}-\bar{\mu} \frac{\phi C_{j} \alpha_{i j}}{\Delta_{f}^{2}} \bar{V}_{r j}$

By comparing (A5.11) with (A4.64) we conclude that:

$-\frac{1}{U_{o}} \int_{S_{f s}} \tau_{f i} \cdot n_{f j} d S=\bar{\mu}^{f} \frac{\phi C_{f} \alpha_{i j}}{\Delta_{f}^{2}} \bar{V}_{r j}^{f}$

Next we evaluate the pressure term. Again using (R.4) we can write:

$$
\frac{1}{U_{o}} \int_{S_{\beta}} p \cdot n_{f j} d S=\phi \frac{\overline{\partial p}}{\partial x_{i}}-\frac{\partial \phi \bar{p}}{\partial x_{i}}
$$

Recall the following two equations: 


$$
\begin{aligned}
& \frac{\overline{\partial p}}{\partial x_{i}}=\frac{\partial \bar{p}}{\partial x_{j}} T^{*}{ }_{j i}-\frac{1}{\phi U_{o}} \int_{S_{\beta}} \rho^{f} V_{i}^{j} \frac{\partial V_{j}^{f}}{\partial x_{i}} x_{i}^{\prime} n_{j} d S-\bar{\rho}^{f} g\left(\delta_{3 i}-T_{3 i}^{*}\right) \\
& \frac{1}{\phi U_{O}} \int_{S_{\beta}} \rho^{f} V_{i}^{f} \frac{\partial V_{j}^{f}}{\partial x_{i}} x_{i}^{\prime} n_{j} d S=-\sqrt{\frac{c_{\nu} \alpha_{m}}{\Delta_{f}^{2}}} \phi \rho^{f}\left|V_{f}-V_{s}\right|\left(V_{f_{i}}-V_{s_{i}}\right)
\end{aligned}
$$

By substituting these two equations into (A5.13) we obtain:

$$
\left.\frac{1}{U_{o}} \int_{S_{f s}} p \cdot n_{j i} d S=\phi \frac{\partial \bar{p}}{\partial x_{j}} T^{*}{ }_{j i}+\phi\right) \sqrt{\frac{c_{v} \alpha_{m}}{\Delta_{f}^{2}}} \rho^{f} \mid V_{f}-V_{s}\left(V_{f_{i}}-V_{s_{i}}\right)-\phi \bar{\rho}^{f} g\left(\delta_{3 i}-T_{3 i}^{*}\right)-\frac{\partial \phi \bar{p}}{\partial x_{i}}
$$

Where the plus sign in front of the Forchheimer term is there because the solid exerts this force to retard fluid flow at the interphase.

By substituting (A5.9), (A5.12) and (A5.14) into (A5.7) we obtain:

$$
\begin{aligned}
& \left.\frac{\partial(1-\phi) \bar{\rho}^{s}\left(\bar{V}_{i}^{s}\right)}{\partial t}=-\frac{\partial}{\partial x_{j}}[1-\phi)\left(\bar{\rho}^{s} \bar{V}_{i}^{s} \bar{V}_{j}^{s}\right)\right] \frac{\partial \bar{\sigma}_{s i j}{ }^{\prime}}{\partial x_{j}}+\bar{\mu}^{f} \frac{\phi C_{f} \alpha_{i j}}{\Delta_{f}^{2}} \bar{V}_{r j}^{f} \\
& +\phi \frac{\partial \bar{p}}{\partial x_{j}} T^{*}{ }_{j i}+\phi \sqrt{\frac{c_{v} \alpha_{m}}{\Delta_{f}^{2}}} \rho^{f}\left|V_{f}-V_{s}\right|_{i}\left(V_{f_{i}}-V_{s_{i}}\right)-\phi \bar{\rho}^{f} g\left(\delta_{3 i}-T_{3 i}^{*}\right)-\frac{\partial \bar{p}}{\partial x_{i}}-(1-\phi) \bar{\rho}^{s} \frac{\partial z}{\partial x_{i}}
\end{aligned}
$$

Which can be further simplified using the equality $\left(\delta_{3 i}-T_{3 i}^{*}\right)=\delta_{3 i}\left(1-T_{j i}^{*}\right)=\frac{\partial z}{\partial x_{i}}\left(1-T_{j i}^{*}\right)$ to obtain:

$$
\begin{aligned}
& \frac{\partial(1-\phi) \bar{\rho}^{s}\left(\bar{V}_{i}^{s}\right)}{\partial t}=-\frac{\partial}{\partial x_{j}}\left[(1-\phi)\left(\bar{\rho} s \bar{V}_{i}^{*} \bar{V}_{j}^{s}\right)\right] \frac{\partial \bar{\sigma}_{s i j}^{\prime}}{\partial x_{j}}+\bar{\mu}^{f} \frac{\phi C_{f} \alpha_{i j}}{\Delta_{s_{f}}^{2}} \bar{V}_{r j}^{f} \\
& +\phi\left(\frac{\partial \bar{p}}{\partial x_{j}}+\bar{\rho}^{f} g \frac{\partial z}{\partial x_{i}}\right) T^{*}{ }_{j i}-\frac{\partial \bar{p}}{\partial x_{i}}-\left(\phi \bar{\rho}^{f}+(1-\phi) \bar{\rho}^{s}\right) \frac{\partial z}{\partial x_{i}}+\sqrt{\frac{c_{v} \alpha_{m}}{\Delta_{f}^{2}}} \phi \bar{\rho}^{f}\left|V_{r}\right| V_{r_{i}}
\end{aligned}
$$

Next we analyze the effective stress tensor.

Bear et al. [1992] presented the following constitutive relationship for the effective stress:

$$
\bar{\sigma}_{s i j}^{\prime}=2 \bar{\mu}^{s} \varepsilon_{s k i j}+\bar{\lambda}^{s} \varepsilon_{s k} \delta_{i j}-\bar{\eta}\left(T_{s}-T_{s 0}\right) \delta_{i j}
$$

Where $\bar{\mu}^{s}, \bar{\lambda}^{s}$, and $\bar{\eta}$ are the Lamé coefficients of the solid matrix that are to be determined experimentally. The skeleton's strain $\varepsilon_{\text {skij }}$ is given by: 


$$
\varepsilon_{s k i j}=\frac{1}{2}\left(\frac{\partial w_{i}}{\partial x_{j}}+\frac{\partial w_{j}}{\partial x_{i}}\right)
$$

Where $\vec{w}$ is the macroscopic displacement vector of the solid skeleton and:

$$
\varepsilon_{s k}=\frac{\partial w_{i}}{\partial x_{i}}
$$

is the volumetric strain.

For equation (A5.17), the solid is assumed to be an isotropic thermoelastic material that only undergoes small deformations.

\section{A6. The energy balance equation for the fluid}

We start this derivation by developing the microscopic energy balance equation for any extensive property $E$. The total energy for a phase within a domain $u$ bounded by a surface $S$ consists of the internal energy (thermodynamic energy) $I$, and the kinetic energy. The energy density $e$ is then given by:

$$
e=\rho I+\frac{1}{2} \rho V^{2}
$$

The energy in the domain $u$ consists of the advective energy flux through the surface $\mathrm{S}$ given by:

$$
\int\left(\rho I+\frac{1}{2} \rho V^{2}\right) V \cdot n d S
$$

and the diffusive heat flux through the surface given by:

$-\int_{S} J^{H} \cdot n d S$

by combining (A6.2) and (A6.3) and applying the divergence theorem we obtain:

$-\int_{u} \nabla \cdot\left\{\rho\left(I+\frac{1}{2} V^{2}\right) V+J^{H}\right\} d U$

Also energy is added by work done by the body forces, which is given by:

$$
\int_{u}^{V} \cdot \rho F d U
$$


and by the surface forces:

$\int_{S}^{V} \cdot \sigma \cdot n d S=\int_{u} \nabla \cdot(V \cdot \sigma) d U$

Finally, by the energy produced within the domain due to chemical reactions is given by:

$\int_{u} \rho \Gamma^{H} d U$

By combining (A6.4) to (A6.7) and letting the volume of the element goes to zero, we obtain the energy balance equation at the microscopic level in the form:

$\frac{\partial}{\partial t}\left(\rho I+\frac{1}{2} \rho V^{2}\right)=-\nabla \cdot\left\{\rho\left(I+\frac{1}{2} V^{2}\right) V+J^{H}\right\}+V \cdot \rho F+\nabla \cdot(V \cdot \sigma)+\rho \Gamma^{H}$

Equation (A6.8) can be written in indicial notation as:

$\frac{\partial}{\partial t}\left(\rho I+\frac{1}{2} \rho V^{2}\right)=-\frac{\partial}{\partial x_{i}}\left\{\rho\left(I+\frac{1}{2} V^{2}\right) V_{i}\right\}-\frac{\partial J_{i}^{H}}{\partial x_{i}}+\rho V_{i} F_{i}+\frac{\partial}{\partial x_{i}}\left(V_{i} \sigma_{i j}\right)+\rho \Gamma^{H}$

With some manipulation to (A6.9) we obtain:

$$
\begin{aligned}
& \rho \frac{D I}{D t}=\sigma_{i j} \frac{\partial V_{i}}{\partial x_{j}}-\frac{\partial J_{i}^{H}}{\partial x_{i}}+\rho \Gamma^{H}-\left(I+\frac{1}{2} V^{2}\right)\left(\frac{\partial \rho}{\partial t}+\frac{\partial \rho V_{i}}{\partial x_{i}}\right) \\
& -V_{i}\left(\frac{\partial \rho V_{i}}{\partial t}+\frac{\partial}{\partial x_{i}}\left\{\rho V_{i} V_{j}\right\}-\frac{\partial \sigma_{i j}}{\partial x_{i}}-\rho F_{i}\right)
\end{aligned}
$$

Next we note that the stress tensor is defined by:

$\sigma_{i j}=\tau_{i j}-p \delta_{i j}$

By substituting (A6.11) into the first term in equation (A6.10) and we assume that the system is non-reactive the later becomes:

$$
\begin{aligned}
& \rho \frac{D I}{D t}=\tau_{i j} \frac{\partial V_{i}}{\partial x_{j}}-p \frac{\partial V_{i}}{\partial x_{i}}-\frac{\partial J_{i}^{H \tilde{H}}}{\partial x_{i}}-\left(I+\frac{1}{2} V^{2}\right)\left(\frac{\partial \rho}{\partial t}+\frac{\partial \rho V_{i}}{\partial x_{i}}\right) \\
& -V_{i}\left(\frac{\partial \rho V_{i}}{\partial t}+\frac{\partial}{\partial x_{i}}\left\{\hat{\omega} V_{i} V_{j}\right\}-\frac{\partial \sigma_{i j}}{\partial x_{i}}-\rho F_{i}\right)
\end{aligned}
$$


Now we desire to express the specific internal energy $I$ in terms of the absolute temperature. To do this, we recall that according the basic principles of thermodynamics, $I=I(v, s)$ where $v$ is the volume and $s$ is the entropy. Thus, for a non-reacting system we can write:

$d I=T d s-p d v$

and from the concept of the total derivative we have:

$d s=\left.\frac{\partial s}{\partial T}\right|_{v} d T+\left.\frac{\partial s}{\partial v}\right|_{T} d v$

Also we use the following Maxwell relationship:

$\left.\frac{\partial s}{\partial v}\right|_{T}=\left.\frac{\partial p}{\partial T}\right|_{v}$

and from (A6.13):

$\frac{\partial I}{\partial T}=T \frac{\partial s}{\partial T}-p \frac{\partial v}{\partial T}$

and for a constant volume, we have:

$\left.\frac{\partial I}{\partial T}\right|_{v}=\left.T \frac{\partial s}{\partial T}\right|_{v}=C_{v}$

Where $C_{v}$ is the specific heat per unit mass at a constant volume assumed constant for our purposes.

By substituting (A6.14) into (A6.13) we obtain:

$d I=\left.T \frac{\partial s}{\partial T}\right|_{\nu} d T+\left(\left.T \frac{\partial s}{\partial v}\right|_{T}-p\right) d v$

and then substituting (A6.15) and (A6.17) into (A6.18) we obtain:

$d I=C_{v} d T+\left(\left.T \frac{\partial p}{\partial T}\right|_{v}-p\right) d v$

Therefore, the R.H.S. of (A6.10) can be written as: 
$\rho \frac{D I}{D t}=\rho C_{v} d T+\rho\left(\left.T \frac{\partial p}{\partial T}\right|_{v}-p\right) \frac{D v}{D t}$

but:

$\rho \frac{D v}{D t}=\rho \frac{D 1 / \rho}{D t}=-\frac{1}{\rho} \frac{D \rho}{D t}$

and we recall the continuity equation:

$$
\begin{aligned}
& \frac{\partial \rho}{\partial t}=-\frac{\partial \rho V_{i}}{\partial x_{i}}=-\rho \frac{\partial V_{i}}{\partial x_{i}}-V_{i} \frac{\partial \rho}{\partial x_{i}} \\
& \frac{\partial \rho}{\partial t}+\rho \frac{\partial V_{i}}{\partial x_{i}}=-\rho \frac{\partial V_{i}}{\partial x_{i}} \\
& \frac{D \rho}{D t}=-\rho \frac{\partial V_{i}}{\partial x_{i}} \\
& -\frac{1}{\rho} \frac{D \rho}{D t}=\frac{\partial V_{i}}{\partial x_{i}}
\end{aligned}
$$

therefore:

$\rho \frac{D I}{D t}=\rho C_{\nu} \frac{D T}{D t}+\left(\left.T \frac{\partial p}{\partial T}\right|_{\nu}-p\right) \frac{\partial V_{i}}{\partial x_{i}}$

By substituting (A6.23) into (A6.12) we obtain:

$$
\begin{aligned}
& \rho C_{v} \frac{D T}{D t}=\tau_{i j} \frac{\partial V_{i}}{\partial x_{j}}-\frac{\partial J_{i}^{H}}{\partial x_{i}}-\left.T \frac{\partial p}{\partial T}\right|_{v} \frac{\partial V_{i}}{\partial x_{i}}-\left(I+\frac{1}{2} V^{2}\right)\left(\frac{\partial \rho}{\partial t}+\frac{\partial \rho V_{i}}{\partial x_{i}}\right) \\
& -V_{i}\left(\frac{\partial \rho V_{i}}{\partial t}+\frac{\partial}{\partial x_{i}}\left\{\rho V_{i} V_{j}\right\}-\frac{\partial \sigma_{i j}}{\partial x_{i}}-\rho F_{i}\right)
\end{aligned}
$$

At this point we cancel the second to last term in (A6.24) using the continuity equation to obtain:

$$
\rho C_{v} \frac{D T}{D t}=\tau_{i j} \frac{\partial V_{i}}{\partial x_{j}}-\frac{\partial J_{i}^{H}}{\partial x_{i}}-\left.T \frac{\partial p}{\partial T}\right|_{\nu} \frac{\partial V_{i}}{\partial x_{i}}-V_{i}\left(\frac{\partial \rho V_{i}}{\partial t}+\frac{\partial}{\partial x_{i}}\left\{\rho V_{i} V_{j}\right\}-\frac{\partial \sigma_{i j}}{\partial x_{i}}-\rho F_{i}\right)
$$

Now we focus on the L.H.S. of (A6.25): 


$$
\begin{aligned}
& \rho C_{v} \frac{D T}{D t}=\rho C_{v}\left(\frac{\partial T}{\partial t}+V_{i} \frac{\partial T}{\partial x_{i}}\right)=\frac{\partial \rho C_{v} T}{\partial t}-T \frac{\partial \rho C_{v}}{\partial t}+\frac{\partial}{\partial x_{i}}\left(\rho C_{v} T V_{i}\right)-T \frac{\partial}{\partial x_{i}}\left(\rho C_{v} V_{i}\right) \\
& =\frac{\partial \rho C_{v} T}{\partial t}+\frac{\partial}{\partial x_{i}}\left(\rho C_{v} T V_{i}\right)-T\left(\frac{\partial \rho C_{v}}{\partial t}+\frac{\partial}{\partial x_{i}}\left(\rho C_{v} V_{i}\right)\right) \\
& =\frac{\partial \rho C_{v} T}{\partial t}+\frac{\partial}{\partial x_{i}}\left(\rho C_{v} T V_{i}\right)-T\left(C_{v} \frac{\partial \rho}{\partial t}+\rho \frac{\partial C_{v}}{\partial t}+\rho V_{i} \frac{\partial}{\partial x_{i}}\left(C_{v}\right)+C_{v} \frac{\partial}{\partial x_{i}}\left(\rho V_{i}\right)\right) \\
& =\frac{\partial \rho C_{v} T}{\partial t}+\frac{\partial}{\partial x_{i}}\left(\rho C_{v} T V_{i}\right)-T\left(C_{v}\left\{\frac{\partial \rho}{\partial t}+\frac{\partial}{\partial x_{i}}\left(\rho V_{i}\right)\right\}+\rho \frac{D C_{v}}{D t}\right) \\
& =\frac{\partial \rho C_{v} T}{\partial t}+\frac{\partial}{\partial x_{i}}\left(\rho C_{v} T V_{i}\right)
\end{aligned}
$$

substituting for the L.H.S. in equation (A6.25) with its value in (A6.26) and rearranging we obtain:

$$
\begin{aligned}
& \frac{\partial \rho C_{v} T}{\partial t}=-\frac{\partial}{\partial x_{i}}\left(\rho C_{v} T V_{i}+J_{i}^{H}\right)-\left.T \frac{\partial p}{\partial T}\right|_{\nu} \frac{\partial V_{i}}{\partial x_{i}}-V_{i}\left(\frac{\partial \rho V_{i}}{\partial t}+\frac{\partial}{\partial x_{i}}\left\{\rho V_{i} V_{j}\right\}-\frac{\partial \sigma_{i j}}{\partial x_{i}}-\rho F_{i}\right) \\
& +\tau_{i j} \frac{\partial V_{i}}{\partial x_{j}}
\end{aligned}
$$

Next, we take the phase average with respect to the liquid continuum of equation (A6.27) as follows

$$
\begin{aligned}
& \phi \overline{\frac{\partial \rho^{f} C^{f} T^{f}}{\partial t}}=-\phi \overline{\frac{\partial}{\partial x_{i}}\left(\rho C^{f} T^{f} V_{i}^{f}+J_{i}^{f H}\right)}-\overline{\left.\phi T^{f} \frac{\partial p}{\partial T^{f}}\right|_{v} \frac{\partial V_{i}^{f}}{\partial x_{i}}}
\end{aligned}
$$

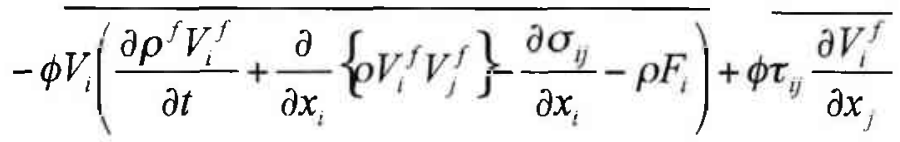

We now analyze each term in equation (A6.28) starting with the L.H.S. and the first term in the R.H.S. combined together:

$$
\left.\phi \overline{\frac{\partial \rho^{f} C^{f} T^{f}}{\partial t}}+\phi \overline{\frac{\partial}{\partial x_{i}}\left(\rho C^{f} T^{f} V_{i}^{f}+J_{i}^{f H}\right.}\right)=\phi \frac{\partial \bar{\rho}^{f} C^{f} \bar{T}^{f}}{\partial t}+\phi \frac{\partial}{\partial x_{i}}\left(\bar{\rho}^{f} C^{f} \bar{T}^{f} \bar{V}_{i}^{f}+\bar{J}_{i}^{f l l}\right)
$$

where we have assumed that:

$$
\left|\bar{\rho}^{f} \bar{T}^{f}\right|>>\left|\overline{\rho^{f} \cdot T^{f}}\right| \text { and }\left|\bar{\rho}^{f} \bar{T}^{f} \bar{V}^{f}\right|>>\left|\overline{\rho^{f} \cdot T^{f} \cdot V^{f}}\right|
$$


and applied averaging rule (R.1), average of a sum.

By applying averaging rules (R.3) and (R.4), averaging of a time derivative and a spatial derivative respectively, we obtain:

$$
\begin{aligned}
& \phi \frac{\partial \bar{\rho}^{f} C^{f} \bar{T}^{f}}{\partial t}+\phi \frac{\partial}{\partial x_{i}}\left(\bar{\rho}^{f} C^{f} \bar{T}^{f} \bar{V}_{i}^{f}+\bar{J}_{i}^{f H}\right)=\frac{\partial}{\partial t}\left(\phi \bar{\rho}^{f} C^{f} \bar{T}^{f}\right)-\int_{S_{f s}} \rho^{f} C^{f} T^{f} u_{i} n_{i} d S \\
& +\frac{\partial}{\partial x_{i}}\left(\phi \bar{\rho}^{f} C^{f} \bar{T}^{f} \bar{V}_{i}^{f}+\bar{J}_{i}^{f H}\right)+\int_{S_{f s}} \rho^{f} C^{f} T^{f} V^{f} n_{i} d S+\int_{S_{f s}} J_{i}^{f H} n_{i} d S \\
& =\frac{\partial}{\partial t}\left(\phi \bar{\rho}^{f} C^{f} \bar{T}^{f}\right)+\frac{\partial}{\partial x_{i}}\left(\phi \bar{\rho}^{f} C^{f} \bar{T}^{f} \bar{V}^{f}+\bar{J}_{i}^{f H}\right)+\int_{S_{f s}} \rho^{f} C^{f} T^{f}\left(V_{i}^{f}-u_{i}\right) n_{i} d S+\int_{S_{f s}} J_{i}^{f H} n_{i} d S
\end{aligned}
$$

Since $S_{f s}$ is a material surface then:

$$
\int_{s_{j s}} \rho^{f} C^{f} T^{f}\left(V_{i}^{f}-u_{i}\right) n_{i} d S=0
$$

and the heat transfer between the two phases through the surface is given by:

$$
\int_{S_{\beta}} J_{i}^{f H} n_{i} d S=\alpha^{* H}\left(\bar{T}^{f}-\bar{T}^{s}\right)
$$

where $\alpha^{* H}$ is a heat transfer coefficient.

By substituting (A6.32) and (A6.33) into (A6.31) we obtain:

$$
\begin{aligned}
& \phi \frac{\partial \bar{\rho}^{f} C^{f} \bar{T}^{f}}{\partial t}+\phi-\frac{\partial}{\partial x_{i}}\left(\bar{\rho}^{f} C^{f} \bar{T}^{f} \bar{V}_{i}^{f}+\bar{J}_{i}^{f H}\right)=\frac{\partial}{\partial t}\left(\phi \bar{\rho}^{f} C^{f f} \bar{T}^{f}\right)+\frac{\partial}{\partial x_{i}}\left(\phi \bar{\rho}^{f} C^{f} \bar{T}^{f} \bar{V}^{f}+\bar{J}_{i}^{f H}\right) \\
& +\alpha^{* H}\left(\bar{T}^{f}-\bar{T}^{s}\right)
\end{aligned}
$$

Next we consider the term:

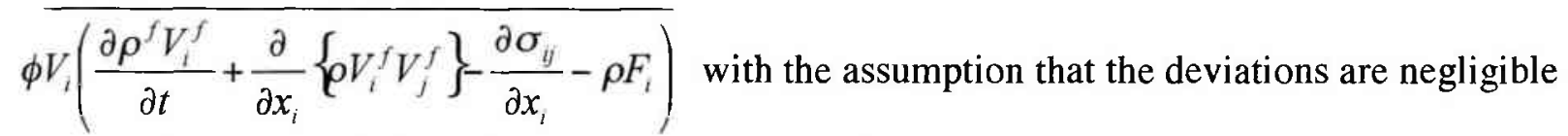
compared to the products of the averages we can write:

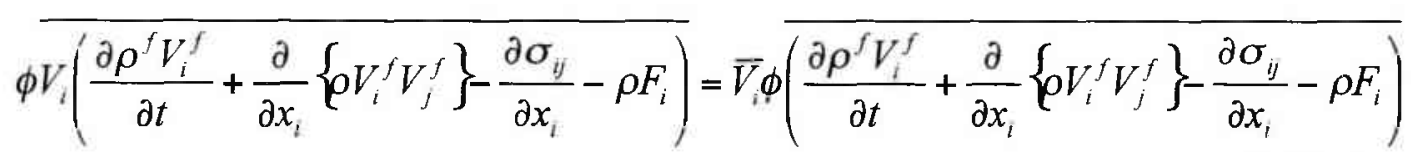

we note that the porosity times the term in the brackets is the average of the momentum balance equation given by: 


$$
\begin{aligned}
& \frac{\partial \phi \overline{\rho^{f}}\left(\overline{V_{i}^{f}}\right)}{\partial t}=-\frac{\partial}{\partial x_{j}}\left(\phi \rho \bar{V}_{i}^{f} \bar{V}_{j}^{f}\right)+\bar{\mu}^{f} \phi \frac{\partial^{2} \bar{V}_{i}^{f}}{\partial x_{j} \partial x_{j}}+\left(\bar{u}^{f}+\bar{\lambda}^{f}\right) \phi \frac{\partial^{2} \bar{V}_{j}^{f}}{\partial x_{i} \partial x_{j}}-\bar{\mu}^{f} \frac{\phi C_{f} \alpha_{i j}}{\Delta_{f}^{2}} \bar{V}_{r j} \\
& -\phi\left(\frac{\partial \bar{p}}{\partial x_{j}}+\bar{\rho}^{f} g \frac{\partial z}{\partial x_{j}}\right) T^{*}{ }_{j i}-\sqrt{\frac{c_{v} \alpha_{m}}{\Delta_{f}^{2}}} \phi \rho^{f}\left|V^{f}-V^{s}\right|\left(V_{i}^{f}-V_{i}^{s}\right)
\end{aligned}
$$

$$
\left.\overline{V_{i} \phi\left(\frac{\partial \rho^{f} V_{i}^{f}}{\partial t}+\frac{\partial}{\partial x_{i}}\left\{\rho V_{i}^{f} V_{j}^{f}\right\}-\frac{\partial \sigma_{i j}}{\partial x_{i}}-\rho F_{i}\right)}\right)=\bar{V}_{i}^{f}\left(\begin{array}{l}
-\bar{\mu}^{f} \phi \frac{\partial^{2} \bar{V}_{i}^{f}}{\partial x_{j} \partial x_{j}}-\left(\bar{\mu}^{f}+\bar{\lambda}^{f} \phi \frac{\partial^{2} \bar{V}_{j}^{f}}{\partial x_{i} \partial x_{j}}\right. \\
\left.+\bar{\mu}^{f} \frac{\phi C_{f} \alpha_{i j}}{\Delta^{2}} \bar{V}_{r j}+\phi\left(\frac{\partial \bar{p}}{\partial x_{j}}+\bar{\rho}^{f} g \frac{\partial z}{\partial x_{j}}\right) \bar{V}_{j}^{f}\right) \\
+\sqrt{\frac{c_{v} \alpha_{m}}{\Delta_{f}^{2}} \phi \rho^{f}}\left|V^{f}-V^{s}\right|\left(V_{i}^{f}-V_{i}^{s}\right)
\end{array}\right)
$$

We desire to write the first two terms in the R.H.S. of (A6.36) in the form:

$$
\frac{\partial \phi \overline{\rho^{f}}\left(\overline{V^{f}}\right)}{\partial t}+\frac{\partial}{\partial x_{j}}\left(\phi \bar{\rho}^{f}\left(\overline{V^{f}}\right) \bar{V}_{j}^{f}\right)
$$

in order for the formulation to be in conserved variables. We do this as follows:

$$
\begin{aligned}
& \frac{\partial \phi \bar{\rho}^{f} \bar{V}_{i}^{f} \bar{V}_{i}^{j}}{\partial t}=\bar{V}_{i}^{f} \frac{\partial \phi \bar{\rho}^{f} \bar{V}_{i}^{f}}{\partial t}+\phi \bar{\rho}^{f} \bar{V}_{i}^{f} \frac{\partial \bar{V}_{i}^{f}}{\partial t} \\
& =\bar{V}_{i}^{f} \frac{\partial \phi \bar{\rho}^{f} \bar{V}_{i}^{f}}{\partial t}+\bar{V}_{i}^{f} \frac{\partial \phi \bar{\rho}^{f} \bar{V}_{i}^{f}}{\partial t}-\bar{V}_{i}^{f} \bar{V}_{i}^{f} \frac{\partial \phi \bar{\rho}^{f}}{\partial t} \\
& =2 \bar{V}_{i}^{f} \frac{\partial \phi \bar{\rho}^{f} \bar{V}_{i}^{f}}{\partial t}-\bar{V}_{i}^{f} \bar{V}_{i}^{f} \frac{\partial \phi \bar{\rho}^{f}}{\partial t}
\end{aligned}
$$

and also: 


$$
\begin{aligned}
& \frac{\partial \phi \bar{\rho}^{f} \bar{V}_{i}^{f} \bar{V}_{i}^{f} \bar{V}_{j}^{f}}{\partial x_{j}}=\bar{V}_{i}^{f} \frac{\partial \phi \bar{\rho}^{f} \bar{V}_{i}^{f} \bar{V}_{j}^{f}}{\partial x_{j}}+\phi \bar{\rho}^{f} \bar{V}_{i}^{f} \bar{V}_{j}^{f} \frac{\partial \bar{V}_{i}^{f}}{\partial x_{j}} \\
& =\bar{V}_{i}^{f} \frac{\partial \phi \bar{\rho}^{f} \bar{V}_{i}^{f} \bar{V}_{j}^{f}}{\partial x_{j}}+\bar{V}_{i}^{f} \frac{\partial \phi \bar{\rho}^{f} \bar{V}_{i}^{f} \bar{V}_{j}^{f}}{\partial x_{j}}-\bar{V}_{i}^{f} \bar{V}_{i}^{f} \frac{\partial \phi \bar{\rho}^{f} \bar{V}_{j}^{f}}{\partial x_{j}} \\
& =2 \frac{\partial \phi \bar{\rho}^{f} \bar{V}_{i}^{f} \bar{V}_{j}^{f}}{\partial x_{j}}-\bar{V}_{i}^{f} \bar{V}_{i}^{f} \frac{\partial \phi \bar{\rho}^{f} \bar{V}_{j}^{f}}{\partial x_{j}}
\end{aligned}
$$

By combining (A6.38) and (A6.39) and noting that:

$$
\begin{aligned}
& -\bar{V}_{i}^{f} \bar{V}_{i}^{f}\left(\frac{\partial \phi \bar{\rho}^{f}}{\partial t}+\frac{\partial \phi \bar{\rho}^{f} \bar{V}_{j}^{f}}{\partial x_{j}}\right)=0 \\
& \frac{\partial \phi \bar{\rho}^{f}\left(\frac{1}{2} \bar{V}^{f}\right)^{2}}{\partial t}+\frac{\partial \phi \bar{\rho}^{f}\left(\frac{1}{2} \bar{V}^{f}\right)^{2} \bar{V}_{j}^{f}}{\partial x_{j}}=\bar{V}_{i}^{f} \frac{\partial \phi \bar{\rho}^{f} \bar{V}_{i}^{f}}{\partial t}+\frac{\partial \phi \bar{\rho}^{f} \bar{V}_{i}^{f} \bar{V}_{j}^{f}}{\partial x_{j}}
\end{aligned}
$$

By substituting (A6.41) into (A6.36) we obtain:

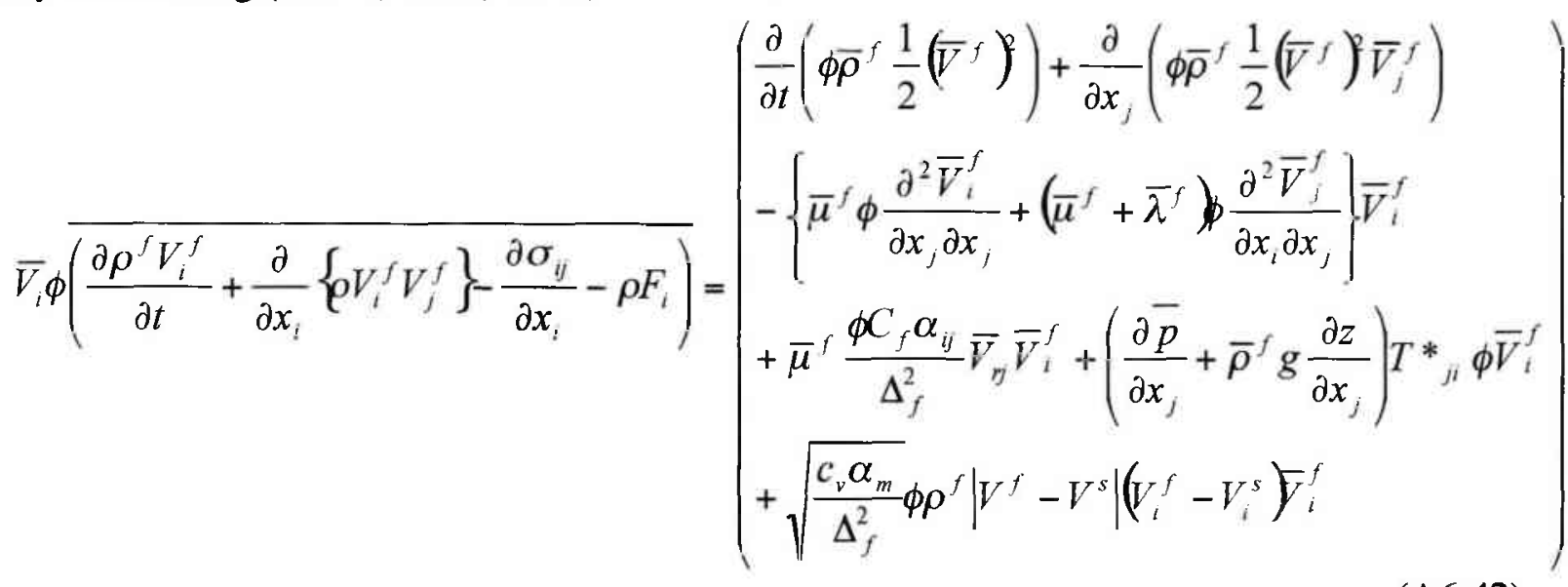

Next we focus on the term $\phi T^{f} \frac{\partial p}{\partial T^{f}} \mid \frac{\partial V_{i}^{f}}{\partial x_{i}}$. With the assumption that:

$\left|\bar{T} f \overline{\frac{\partial p}{\partial T^{f}}}\right|_{v} \frac{\overline{\partial V_{i}^{f}}}{\partial x_{i}}|\gg| T^{f},\left.\frac{\partial p}{\partial T^{f}}\right|_{v}, \frac{\partial V_{i}^{f}}{\partial x_{i}} \mid$

we can write:

$$
\left.\phi T^{f} \frac{\partial p}{\partial T^{f}}\right|_{\nu} \frac{\partial V_{i}^{f}}{\partial x_{i}}=\bar{T}^{f} \overline{\left.\frac{\partial p}{\partial T^{f}}\right|_{\nu}} \phi \frac{\overline{\partial V_{i}^{f}}}{\partial x_{i}}
$$

and by using averaging rule (R.4) we can write:

$$
\bar{T}^{f} \frac{\overline{\partial p}}{\partial T^{f}} \phi_{\nu} \phi \frac{\overline{\partial V_{i}^{f}}}{\partial x_{i}}=\left.\bar{T}^{f} \frac{\overline{\partial p}}{\partial T^{f}}\right|_{\nu}\left(\frac{\partial \phi \bar{V}_{i}^{f}}{\partial x_{i}}+\frac{1}{U_{o}} \int_{S_{s s}} V_{i}^{f} n_{i} d S\right)
$$

Now by assuming that the rate of change of velocity within the fluid is not significantly influenced by the interaction at the solid-fluid interface, i.e,.: 
$\left|\frac{\partial \phi \bar{V}_{i}^{f}}{\partial x_{i}}\right| \gg\left|\frac{1}{\bar{U}_{o}} \int_{S_{\text {f }}} V_{i}^{f} n_{i} d S\right|$

then equation (A6.45) takes the form:

$\left.\left.\bar{T}^{f} \overline{\frac{\partial p}{\partial T^{f}}}\right|_{\nu} \underset{\frac{\partial V_{i}^{f}}{\partial x_{i}}}{=\bar{T}^{f}} \frac{\overline{\partial p}}{\partial T^{f}}\right|_{\nu} \frac{\partial \phi \bar{V}_{i}^{f}}{\partial x_{i}}$

we recall that the density of any fluid is a function of temperature and pressure (at a fixed concentration) therefore we can write:

$d \rho=\left.\frac{\partial \rho}{\partial p}\right|_{T} d P+\left.\frac{\partial \rho}{\partial T}\right|_{p} d T$

For a constant density equation (A6.48) takes the form:

$\left.\frac{\partial \rho}{\partial p}\right|_{T} d P+\left.\frac{\partial \rho}{\partial T}\right|_{P} d T=0$

$\frac{d P}{d T}=\frac{-\left.\frac{\partial \rho}{\partial T}\right|_{p}}{\left.\frac{\partial \rho}{\partial p}\right|_{T}}=\frac{-\left.\frac{1}{\rho} \frac{\partial \rho}{\partial T}\right|_{p}}{\left.\frac{1}{\rho} \frac{\partial \rho}{\partial p}\right|_{T}}=\left.\frac{\beta_{T}}{\beta_{p}}\right|_{\rho}$

where $\beta_{T}$ is the coefficient of thermal expansion of the fluid at constant pressure and $\beta_{p}$ is the coefficient of compressibility of the fluid.

By substituting (A6.49) into (A6.47) we obtain:

$\left.\bar{T}^{f} \overline{\frac{\partial p}{\partial T^{f}}}\right|_{\nu} \phi \frac{\overline{\partial V_{i}^{f}}}{\partial x_{i}}=\left.\bar{T}^{f} \frac{\overline{\beta_{T}}}{\beta_{p}}\right|_{\rho} \frac{\partial \phi \bar{V}_{i}^{f}}{\partial x_{i}}$

Bear and Bachmat [1990], following a development from Fourier's Law determined that the averaged heat flux term $\bar{J}_{i}^{f H}$ has the form:

$\bar{J}_{i}^{f H}=-\phi \lambda_{i j}^{*} \frac{\partial \bar{T}_{i}^{f}}{\partial x_{i}}$

Next we consider the term $\phi \tau_{i j} \frac{\partial V_{i}^{j}}{\partial x_{j}}$ and we assume that:

$\left|\overline{\bar{\tau}}_{i j} \phi \overline{\frac{\partial V_{i}^{f}}{\partial x_{j}}}\right|>>\left|\overline{\boldsymbol{\tau}_{i j}^{\prime} \frac{\partial V_{i}^{f \prime}}{\partial x_{j}}}\right|$

With this assumption and using averaging rule (R.4) we obtain:

$\bar{\tau}_{i j} \phi \frac{\overline{\partial V_{i}^{f}}}{\partial x_{j}}=\bar{\tau}_{i j}\left(\frac{\partial \phi \bar{V}_{i}^{f}}{\partial x_{j}}+\frac{1}{U_{o}} \int_{S_{f s}} V_{i}^{f} n_{j} d S\right)$

recalling equation (A4.46):

$\frac{1}{U_{o}} \int_{S_{i s}} V_{i} n_{j} d S=-\bar{V}_{s i} \frac{\partial \phi}{\partial x_{j}}=\phi \frac{\partial \bar{V}_{s i}}{\partial x_{j}}-\frac{\partial \phi \bar{V}_{s i}}{\partial x_{j}}$ 
we can rewrite (A6.53) as follows:

$\bar{\tau}_{i j} \phi \frac{\overline{\partial V_{i}^{f}}}{\partial x_{j}}=\bar{\tau}_{i j}\left(\frac{\partial \phi{\overline{V_{i}^{f}}}^{f}}{\partial x_{j}}+\phi \frac{\partial{\overline{V_{i}}}^{s}}{\partial x_{j}}-\frac{\partial \phi{\overline{V_{i}}}_{i}^{s}}{\partial x_{j}}\right)$

Next we recall that:

$\tau_{i j}=\mu\left(\frac{\partial V_{i}}{\partial x_{j}}+\frac{\partial V_{j}}{\partial x_{i}}\right)+\lambda\left(\frac{\partial V_{k}}{\partial x_{k}}\right) \delta_{i j}$

By averaging (A4.1) we obtain:

$\bar{\tau}_{i j}=\bar{\mu}^{f}\left(\frac{\partial \bar{V}_{i}}{\partial x_{j}}+\frac{\partial \bar{V}_{j}}{\partial x_{i}}\right)+\bar{\lambda}^{f}\left(\frac{\partial \bar{V}_{k}}{\partial x_{k}}\right) \delta_{i j}$

therefore:

$\bar{\tau}_{i j} \phi \frac{\overline{\partial V_{i}^{f}}}{\partial x_{j}}=\left(\bar{\mu}^{f}\left(\frac{\partial \bar{V}_{i}}{\partial x_{j}}+\frac{\partial \bar{V}_{j}}{\partial x_{i}}\right)+\bar{\lambda}^{f}\left(\frac{\partial \bar{V}_{k}}{\partial x_{k}}\right) \delta_{i j}\right)\left(\frac{\partial \phi \bar{V}_{i}^{f}}{\partial x_{j}}+\phi \frac{\partial \bar{V}_{i}^{s}}{\partial x_{j}}-\frac{\partial \phi \bar{V}_{i}^{s}}{\partial x_{j}}\right)$

Finally by substituting (A6.34), (A6.42), (A6.50), (A6.51), and (A6.53) into (A6.28) we obtain the energy balance equation for the fluid phase:

$$
\begin{aligned}
& \frac{\partial}{\partial t}\left(\phi \bar{\rho}^{f}\left\{C^{f} \bar{T}^{f}+\frac{1}{2}\left(\bar{V}^{f}\right)^{f}\right\}\right)=-\frac{\partial}{\partial x_{i}}\left(\phi \bar{\rho}^{f}\left\{C^{f} \bar{T}^{f}+\frac{1}{2}\left(\bar{V}^{f}\right)^{f}\right\} \bar{V}_{i}^{f}-\phi \lambda_{i j}^{*} \frac{\partial \bar{T}^{f}}{\partial x_{i}}\right) \\
& -\alpha^{* H}\left(\bar{T}^{f}-\bar{T}^{s}\right)-\bar{T}^{f} \frac{\beta_{T}}{\beta_{p}} \mid \rho \frac{\partial \phi \bar{V}_{i}^{f}}{\partial x_{i}}+\left\{\bar{\mu}^{f} \phi \frac{\partial^{2} \bar{V}_{i}^{f}}{\partial x_{j} \partial x_{j}}+\left(\bar{\mu}^{f}+\bar{\lambda}^{f}\right\rangle \frac{\partial^{2} \bar{V}_{j}^{f}}{\left.\partial x_{i} \partial x_{j}\right\}} \bar{V}_{i}^{f}\right. \\
& -\bar{\mu}^{f} \frac{\phi C_{f} \alpha_{i j}}{\Delta_{f}^{2}} \bar{V}_{r j} \bar{V}_{i}^{f}-\left(\frac{\partial \bar{p}}{\partial x_{j}}+\bar{\rho}^{f} g \frac{\partial z}{\partial x_{j}}\right) T^{*}{ }_{j i} \phi \bar{V}_{i}^{f} \\
& -\sqrt{\frac{c_{v} \alpha_{m}}{\Delta_{f}^{2}} \phi \rho^{f}}\left|V^{f}-V^{s}\right|\left(V_{i}^{f}-V_{i}^{s} \bar{Y}_{i}^{f}+\bar{\tau}_{i j} \phi \frac{\overline{\partial V_{i}^{f}}}{\partial x_{j}}\right.
\end{aligned}
$$

\section{A7. The Energy balance equation for the solid phase}

Following a similar development to that used in deriving the fluid's microscopic energy balance equation for an isotropic thermoelastic solid we obtain:

$$
\frac{\partial \rho C_{v} T}{\partial t}=-\frac{\partial}{\partial x_{i}}\left(\rho C^{s} T V_{i}+J_{i}^{I i}\right)-T \eta \frac{\partial \varepsilon}{\partial t}-V_{i}\left(\frac{\partial \rho V_{i}}{\partial t}+\frac{\partial}{\partial x_{i}}\left\{\rho V_{i} V_{j}\right\}-\frac{\partial \sigma_{i j}}{\partial x_{i}}-\rho F_{i}\right)
$$


Where the term $C^{s}$ is the specific heat of the solid at a constant strain, $\eta$ is a coefficient relating the temperature variation to the change in the stress of the solid.

Next we average (A7.1) with respect to the solid continuum as follows:

$$
\begin{aligned}
& (1-\phi) \frac{\overline{\partial \rho C_{v} T}}{\partial t}+(1-\phi) \overline{\frac{\partial}{\partial x_{i}}\left(\rho C^{s} T V_{i}+J_{i}^{H}\right)}=-(1-\phi) \overline{I \eta \frac{\partial \varepsilon}{\partial t}} \\
& -(1-\phi) \boldsymbol{V}_{i}\left(\frac{\partial \rho V_{i}^{\prime}}{\partial t}+\frac{\partial}{\partial x_{i}}\left\{\rho V_{i} V_{j}\right\}-\frac{\partial \sigma_{i j}}{\partial x_{i}}-\rho F_{i}\right)
\end{aligned}
$$

First we consider the terms on the L.H.S., by assuming that

$$
\left|\bar{\rho}^{s} \bar{T}^{s}\right| \gg\left|\overline{\rho^{s \prime} T^{s}}\right| \text { and }\left|\bar{\rho}^{s} \bar{T}^{s} \bar{V}^{s}\right|>>\sqrt{\rho^{s \prime} T^{s t} V^{s}} \mid
$$

and applying averaging rule (R.1), average of a sum, we obtain:

$$
\begin{aligned}
& \left.(1-\phi) \frac{\overline{\partial \rho^{s} C^{s} T^{s}}}{\partial t}+(1-\phi) \frac{\partial-\frac{\partial}{\partial x_{i}}\left(\rho C^{s} T^{s} V_{i}^{s}+J_{i}^{s H}\right.}{\partial t}\right)=(1-\phi) \frac{\partial \bar{\rho}^{s} C^{s} \bar{T}^{s}}{\partial t} \\
& +(1-\phi) \frac{\partial}{\partial x_{i}}\left(\bar{\rho}^{s} C^{s} \bar{T}^{s} \bar{V}_{i}^{s}+\bar{J}_{i}^{s H}\right)
\end{aligned}
$$

By applying averaging rules (R.3) and (R.4), averaging of a time derivative and a spatial derivative respectively, we obtain:

$$
\begin{aligned}
& (1-\phi) \frac{\partial \bar{\rho}^{s} C^{s} \bar{T}^{s}}{\partial t}+(1-\phi) \frac{\partial}{\partial x_{i}}\left(\bar{\rho}^{s} C^{s} \bar{T}^{s} \bar{V}_{i}^{s}+\bar{J}_{i}^{s H}\right)=\frac{\partial}{\partial t}\left((1-\phi) \bar{\rho}^{s} C^{s} \bar{T}^{s}\right)-\int_{s_{s}} \rho^{s} C^{s} T^{s} u_{i} n_{i} d S \\
& +\frac{\partial}{\partial x_{i}}\left((1-\phi) \bar{\rho}^{s} C^{s} \bar{T}^{s} \bar{V}_{i}^{s}+\bar{J}_{i}^{s H}\right)+\int_{S_{A}} \rho^{s} C^{s} T^{s} V^{s} n_{j} d S+\int_{S_{s s}} J_{i}^{s H} n_{i} d S \\
& =\frac{\partial}{\partial t}\left((1-\phi) \bar{\rho}^{s} C^{s} \bar{T}^{s}\right)+\frac{\partial}{\partial x_{i}}\left((1-\phi) \bar{\rho}^{s} C^{s} \bar{T}^{s} \bar{V}_{i}^{s}+\bar{J}_{i}^{s H}\right)+\int_{s_{s}} \rho^{s} C^{s} T\left(V_{i}^{s}-u_{i}\right) r_{i} d S+\int_{s_{s s}} J_{i}^{s H} n_{i} d S
\end{aligned}
$$

Since $S_{f s}$ is a material surface then:

$$
\int_{S_{f s}} \rho^{s} C^{s} T\left(V_{i}^{s}-u_{i}\right) n_{i} d S=0
$$

Here we note that the heat flux through the surface between the solid and the fluid must be equal in magnitude and opposite in sign, thus from equation

$$
\int_{S_{f s}} J_{i}^{f I I} n_{i} d S=\alpha^{* H}\left(\bar{T}^{f}-\bar{T}^{s}\right)
$$


we can write:

$\int_{S_{j s}} J_{i}^{s H} n_{i} d S=-\alpha^{* H}\left(\bar{T}^{f}-\bar{T}^{s}\right)$

By substituting (A7.6) and (A7.7) into (A7.5) we obtain:

$$
\begin{aligned}
& (1-\phi) \frac{\partial \bar{\rho}^{s} C^{s} \bar{T}^{s}}{\partial t}+(1-\phi) \frac{\partial}{\partial x_{i}}\left(\bar{\rho}^{s} C^{s} \bar{T}^{s} \bar{V}_{i}^{s}+\bar{J}_{i}^{s H}\right)=\frac{\partial}{\partial t}\left((1-\phi) \bar{\rho}^{s} C^{s} \bar{T}^{s}\right) \\
& +\frac{\partial}{\partial x_{i}}\left((1-\phi) \bar{\rho}^{s} C^{s} \bar{T}^{s} \bar{V}_{i}^{s}+\bar{J}_{i}^{s H}\right)-\alpha^{* H}\left(\bar{T}^{f}-\bar{T}^{s}\right)
\end{aligned}
$$

Next we consider the average of the term including the momentum balance equation. By following a procedure similar to that used for the derivation of the fluid's energy balance equation and by recalling that

$$
\begin{aligned}
& \frac{\partial(1-\phi) \bar{\rho}^{s}\left(\bar{V}_{i}^{s}\right)}{\partial t}=-\frac{\partial}{\partial x_{j}}\left[(1-\phi)\left(-s \bar{V}_{i} ; \bar{V}_{j}^{s}\right)\right] \frac{\partial \bar{\sigma}_{s i j}{ }^{\prime}}{\partial x_{j}}+\bar{\mu}^{f} \frac{\phi C_{f} \alpha_{i j}}{\Delta_{f}^{2}} \bar{V}_{r j}^{f} \\
& +\phi\left(\frac{\partial \bar{p}}{\partial x_{j}}+\bar{\rho}^{f} g \frac{\partial z}{\partial x_{i}}\right) T^{*}{ }_{j i}-\frac{\partial \bar{p}}{\partial x_{i}}-\left(\phi \bar{\rho}^{f}+(1-\phi) \bar{\rho}^{s}\right) \frac{\partial z}{\partial x_{i}}-\sqrt{\frac{c_{v} \alpha_{m}}{\Delta_{f}^{2}}} \phi \bar{\rho}^{f}\left|V_{r}\right| V_{r_{i}}
\end{aligned}
$$

is the macroscopic momentum balance equation for the solid we obtain:

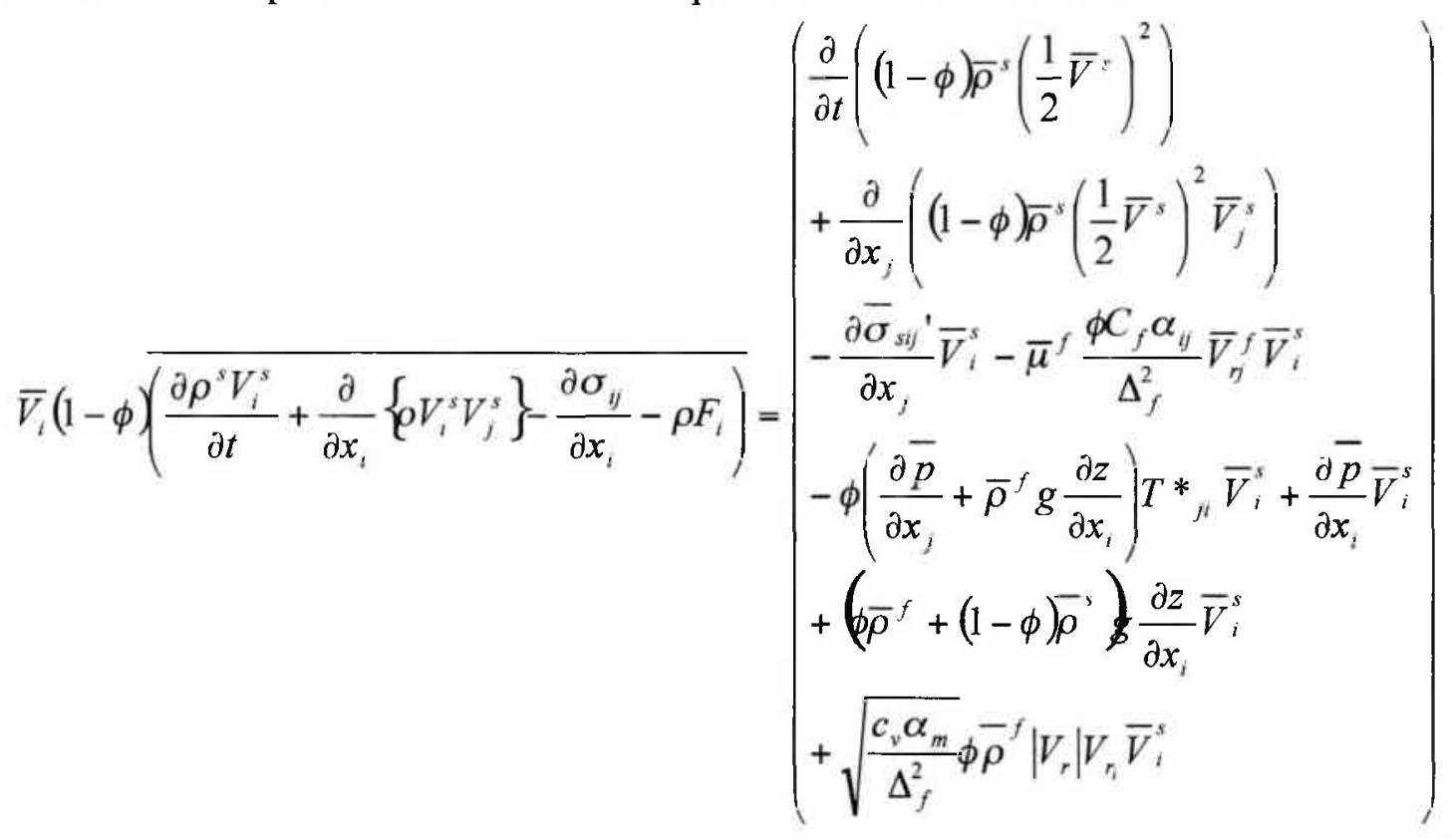

Next we consider the term $(1-\phi) \overline{I \eta \frac{\partial \varepsilon}{\partial t}}$. By assuming that 


$$
(1-\phi) \overline{\Gamma \eta \frac{\partial \varepsilon}{\partial t}} \cong \bar{T}^{s} \bar{\eta}(1-\phi) \overline{\frac{\partial \varepsilon}{\partial t}}
$$

and that the strain at the surface is mainly tangential in direction we can write:

$$
(1-\phi) \overline{T \eta \frac{\partial \varepsilon}{\partial t}} \cong \bar{T} \bar{\eta} \frac{\partial(1-\phi) \bar{\varepsilon}}{\partial t}
$$

Also the conductive heat flux through the solid is given by:

$$
\bar{J}_{i}^{s H}=-\phi \lambda_{i j}^{* s} \frac{\partial \bar{T}_{i}^{s}}{\partial x_{i}}
$$

To obtain the energy balance equation for the solid phase we substitute (A7.8), (A7.9), (A7.11), and (A7.12) into (A7.2) and rearranging we obtain:

$$
\begin{aligned}
& \frac{\partial}{\partial t}\left((1-\phi) \bar{\rho}^{s}\left\{C^{s} \bar{T}^{s}+\frac{1}{2}\left(\bar{V}^{s}\right)^{2}\right\}\right)=-\frac{\partial}{\partial x_{i}}\left((1-\phi) \bar{\rho}^{s}\left\{C^{s} \bar{T}^{s}+\frac{1}{2}\left(\bar{V}^{s}\right)^{2}\right\} \bar{V}_{i}^{s}-\phi \lambda_{i j}^{* s} \frac{\partial \bar{T}_{i}^{s}}{\partial x_{i}}\right) \\
& +\alpha^{* H}\left(\bar{T}^{f}-\bar{T}^{s}\right)-\bar{T}^{s} \bar{\eta} \frac{\partial(1-\phi) \bar{\varepsilon}}{\partial t}+\frac{\partial \bar{\sigma}_{s i j}^{\prime} \bar{V}_{i}^{s}+\bar{\mu}^{f} \frac{\phi C_{f} \alpha_{i j}}{\partial x_{j}} \bar{V}_{r j}^{f} \bar{V}_{i}^{s}}{+\phi\left(\frac{\partial \bar{p}}{\partial x_{j}}+\bar{\rho}^{f} g \frac{\partial z}{\partial x_{i}}\right) T^{*}{ }_{j i} \bar{V}_{i}^{s}-\frac{\partial \bar{p}}{\partial x_{i}} \bar{V}_{i}^{s}-\left(\phi \bar{\rho}^{f}+(1-\phi) \bar{\rho}^{s}\right) \frac{\partial z}{\partial x_{i}} \bar{V}_{i}^{s}} \\
& -\sqrt{\frac{c_{v} \alpha_{m}}{\Delta_{f}^{2}} \phi \bar{\rho}^{f}\left|V_{r}\right| V_{r_{i}} \bar{V}_{i}^{s}}
\end{aligned}
$$

\section{References}

Bear, J. and Bachmat, Y., 1986, "Macroscopic Modeling of Transport Phenomena in Porous Media. 2: Application to Mass, Momentum and Energy Transport," Transport in Porous Media, 1, 1986, pp. 241-269.

Bear, J. and Bachmat, Y., 1990, Introduction to Modeling of Transport Phenomena in Porous Media, Kluwer, Dordrecht, The Netherlands.

Bear, J., Sorek, S., Ben-Dor, G., and Mazor, G., 1992, "Displacement waves in saturated thermoelastic porous media. I. Basic equations." Fluid Dynamics Research, 9, 155-164.

Hsu, C. T., and Cheng, P., 1990, "Thermal dispersion in a porous medium," International Journal of Heat and Mass Transfer, 33, 1587-1597.

Levy, A., Sorek, S., Ben-Dor, G., and Bear, J., 1995, "Evolution of the balance equations in saturated thermoelastic porous media following abrupt simultaneous changes in pressure and temperature," Transport in Porous Media, 21, 241-268. 
Nield, D. A., 1991, "The limitations of the Brinkman-Forchheimer equation in modeling flow in a saturated porous medium and at an interface," International Journal of Heat and Fluid Flow, 12, 269-272.

Terzaghi, K., 1925, Erdbaumeckanik auf bodenphysikalische Grundlage (Deuticke, Leipzig). 


\section{Appendix C. Literature Review of Production Formations}

\section{Glade Formation}

Introduction

The following information was taken from Mineral Resources Report M52 entitled "Oil and Gas Geology of the Warren Quadrangle, Pennsylvania." This document was published in 1965 by William S. Lytle of the Pennsylvania Geological Survey. Additional information was found in Mineral Resources Report M53 entitled "Oil and Gas Geology of the Youngsville Quadrangle, Pennsylvania." This document was published in 1964 by William G. McGlade of the Pennsylvania Geological Survey.

The Warren and Youngsville quadrangles are located in Warren County of northwestern Pennsylvania. The Warren quadrangle area is about 222 square miles. About one third is productive of oil and gas. The main producing sands are the Glade, Clarendon Stray, Clarendon, Gartland, and Cherry Grove of Upper Devonian age. The Youngsville quadrangle occupies the north-central portion of Warren County adjacent to the New York State Line. As of September 30, 1963, approximately 535 oil wells and/or gas wells were producing from the Glade sandstone in the Youngsville quadrangle. The first wells were drilled in this area as early as 1865 .

The term "sand" in this report refers to potentially productive zones of mostly sandstone with associated siltstones and shales. The terms "formation" or "rocks" in this report are more inclusive than the term "sand" and include transitional zones above and below the producing zone. The term "sandstone" refers only to beds of lithified sand.

Glade Formation

In the Warren quadrangle, the top of the Glade rocks lies at about 200 feet below the base of the "Pink Rock" or 75 feet below the base of the Warren Second sand. These rocks extend about 125 feet to the top of the Clarendon formation. The base of the Glade formation is also the base of the Conneaut Group and the top of the Canadaway Group, and is separated from the overlying Warren Second sand by approximately 75 feet of shale, sandstone, and siltsone. The Glade formation strata may be subdivided into three lithologic units (top to bottom): Glade siltstone, Glade sandstone (the producing zone) and the Glade transitional zone. The lower unit usually has a shale body at the base for convenient separation from the underlying sandy Clarendon formation.

The top of the Glade siltsone is the beginning of a silty section about 100 feet below the Warren Second sand. This predominantly greenish-gray slightly calcareous, argillaceous siltstone contains fossils and some interbedded greenish-gray shale. The Glade siltstone is about 40 feet thick. In areas where the Glade sand reaches maximum thickness, the sand extends upward and replaces part of the Glade siltstone. Over most of the oil and gas producing area the Glade siltstone is a consistent lithologic unit. The top of the Glade siltstone can readily be found from samples and on geophysical logs.

The middle zone is the Glade sand which is one of the main producing zones of the Warren quadrangle. This sand is mostly a very fine- to fine-grained, light-gray sandstone. In some 
productive areas the Glade is a greenish-gray siltstone and is also slightly micaceous and calcareous. Fossil fragments can be found in the middle of the sandstone occasionally. The productive Glade sandstone of the Glade formation in the Youngsville quadrangle occurs as a bar-like sandstone body which trends slightly east of north direction through the eastern portion of the quadrangle. This sandstone appears to thin gradually between the town of Sugar Grove and the southeastern end of the quadrangle. A thicker Glade sandstone, thought to be the same unit, seems to continue northward into Chautauqua County, New York.

\section{Historical Review}

In 1881, an oil well presumed to be from the Glade sandstone was completed near Youngsville. A considerable number of wells were drilled between 1910 and 1915 in Youngsville, Matthews Run, and in the Five Points area. After 1915, activity tapered off for several years. The Sugar Grove Gas Pool was drilled in 1925 and 1926. Almost all of the wells were either abandoned or became inoperative by the late 1920's except the wells in the Youngsville Pool and the Sugar Grove Gas Pool. Drilling was resumed in the Youngsville Pool and the Five Points Pools about 1929. In both these pools a pilot re-pressuring project was attempted. The projects were abandoned after no success. Sparse drilling continued in the Five Points Pools until 1946. Drilling activity was vigorously renewed in 1946 and about 30 wells were drilled during the year in the Sugar Grove, Five Points, and Chandlers Valley Pools. Between 1947 and 1962 operators drilled several wells each year in the Youngsville-Sugar Grove Field.

In 1949, a pilot re-pressuring project was conducted near Sugar Grove. Selected portions of the Glade sandstone were shot with nitroglycerine and then subjected periodically to injection pressures of 350 pounds per square inch during the following three years. Very little gas was injected and no increase in oil production was obtained.

Before 1962, shooting with nitroglycerin was the accepted method of completion in the Glade reservoir and all other reservoirs in the Youngsville quadrangle. Two wells within the Sugar Grove Pool were hydraulically fractured before 1953 using kerosene and sand. Neither well responded to the treatment. The use of hydraulic fracturing as a stimulation method in the Sugar Grove Pool was suspended for more than ten years.

During October, 1961, the United States Bureau of Mines, in cooperation with Howard Curtis and Son Co., cut and analyzed a 4-inch diameter rotary air core of the Glade sandstone. The well owner was advised in January, 1962 by the Bureau of Mines that hydraulically fracturing the Glade sandstone would be advantageous. A well that had been shot with 100 quarts of nitroglycerin was fractured by Curtis. This well produced satisfactory results, leading to the future method of Glade sandstone development.

\section{Stratigraphy}

A three-well correlation among wells in Tidioute area, the Youngsville area and the Warren quadrangle shows the following thicknesses of the Glade formation.

$\begin{array}{ll}\text { Glade siltstone } & 56 \mathrm{feet} \\ \text { Glade sandstone } & 20 \mathrm{feet} \\ \text { Glade transitional zone } & 74 \mathrm{feet}\end{array}$


The Glade formation of the Youngsville quadrangle is the stratigraphic equivalent of the Queen formation of the Tidioute and Sheffield quadrangles, as well as the "Eighty Foot rock" and the Glade sand pay of the Warren area. The Queen formation was named after the village of Queen located in the Tidioute quadrangle. The Glade sandstone was named from a subsurface section of an oil well located at Glade, Glade Township in the Warren quadrangle in March, 1875 . One author noted that the Glade sandstone of the Warren quadrangle might correlate with the Bradford First sand in McKean County which is approximately correlative with the Cuba sandstone near Cuba, Allegany County, New York.

As of 1963, the Glade unit could be traced from a point just north of the New York-Pennsylvania border to south of the town of Tidioute, along the Allegheny River. This sandstone body was difficult to trace south of the Youngsville quadrangle due to the lack of detailed subsurface data.

The Glade siltstone is usually about 45 to 55 feet thick. Consisting mainly of light-gray to medium gray, sometimes-calcereous siltstone interbedded with thin beds of dark-gray shale, the siltstone is overlain by a dark-gray silty shale unit. Usually about 80 feet thick, the shale unit separates the Warren Second sand from the Glade rocks.

\author{
Lithologic Descriptions of Glade "sandstone" cores \\ Youngsville and Warren Quadrangles, Pennsylvania \\ Howard Curtis and Son Co. \\ Sandene Lease Well No. 14
}

Core description by William Nabors (697-748.6) and William K. Overbey, Jr. (749-771) both of the Morgantown Petroleum Research Laboratory, Bureau of Mines, United States Department of the Interior-with modifications by W.G. McGlade.

\begin{tabular}{|c|c|c|}
\hline Depth, $f t$ & \multicolumn{2}{|c|}{ Thickness } \\
\hline Top Bottom & Feet & Lithologic description \\
\hline $697.47-697.9$ & 0.43 & Shale, calcareous, dark-gray \\
\hline $697.9-700.0$ & 0.1 & Shale, calcareous, silty, green \\
\hline $700.0-705.0$ & 5.0 & Sandy shale, medium-gray \\
\hline $705.0-708.8$ & 3.8 & Sandstone, fine-grained, shaly, calcareous, light-gray, hard \\
\hline $708.8-714.0$ & 5.2 & Sandstone, fine-grained, light-gray, micaceous, hard \\
\hline $714.0-716.5$ & 2.5 & Sandstone, fine-grained, light-gray, hard slight oil bleeding \\
\hline $716.5-717.41$ & 0.91 & Sandstone, fine-grained, light-gray, hard, light oil bleeding \\
\hline $717.41-717.85$ & 0.44 & Sandstone, fine-grained, light-gray, hard, heavy oil bleeding \\
\hline $717.85-720.3$ & 6.45 & $\begin{array}{l}\text { Sandstone, fine-grained, with limestone laminae, light-gray, hard, } \\
\text { heavy oil bleeding }\end{array}$ \\
\hline $720.3-726.0$ & 5.7 & $\begin{array}{l}\text { Sandstone, fine-grained, light-gray, hard, limestone laminae } \\
\text { heavy oil bleeding }\end{array}$ \\
\hline $724.65-724.8$ & 0.15 & Shale laminae \\
\hline
\end{tabular}


- -725.2

725.4-725.55

$725.75-725.950 .2$

726.0-726.5

726.5-730.3

$730.0-730.3$

730.3-735.85

732.4-735.85

735.85-736.49

$736.49-739.2$

739.2-739.9

739.9-741.4

$741.4-741.8$

$741.8-742.8$

742.8-745

$745.0-746.0$

$746.0-748.6$

$749-754$

$754-757$

$757-764$

764-769

$769-770$

770-771
- Shale pebbles

0.15 Shale

Horizontal fractures about $1 / 4$ inch between fractures

0.5 Sandstone, fine-grained, light-gray, slightly micaceous, hard, irregular shale laminae, heavy oil bleeding

3.8 Sandstone, fine-grained, light-gray, hard, heavy oil bleeding

0.3 Irregular shale laminae

5.55 Sandstone, fine-grained, light-gray, hard excessive oil bleeding

3.45 Fossiliferous zone

0.64 Sandstone, very fine-grained, light-gray, bubbling oil show

2.71 Sandstone, fine-grained, light-gray, hard, slight oil bleeding

0.7 Sandstone, fine-grained, light-gray, hard, fossiliferous, slight oil bleeding

1.5 Sandstone, fine-grained, light-gray, hard, oil odor

0.4 Sandstone, fine-grained, laminated, light-gray, hard, oil show

1.0 Sandstone, fine-grained, light-gray, hard, slight oil bleeding

2.2 Sandstone, fine-grained, highly laminated, light-gray, hard, oil odor

1.0 Sandstone, fine-grained, silty, micaceous, light-gray

2.6 Sandstone, fine-grained, silty, light-gray, medium hard

5.0 Sandstone, fine-grained, subangular, micaceous, slightly argillaceous and dolomitic, light-gray to white. Traces of Montmorillonite clay by benzidine stain. Fossiliferous in zones

3.0 Sandstone, very fine-grained, light-gray to white, interbedded within thin shale streaks

7.0 Sandstone, fine-grained, light-gray to white, micaceous, many thin clay and shale partings, some up to 1 inch thick

5.0 Shale, sandy, medium-gray to dark-gray, micaceous, with thin sandstone interbeds up to 1 inch thick

1.0 Limestone, very sandy, medium-gray, very argillaceous, very fossiliferous

1.0 Sandstone, very fine-grained, white to light-gray, calcareous, fossiliferous 


\section{CORE ANALYSIS, GLADE SANDSTONE}

Howard Curtis and Son Co., Sandene Lease Well No. 14

Northwest Section 3, Youngsville quadrangle

Sugar Grove Township, Warren County, Pennsylvania

Date cored: October, 1961

Cored interval:

$697^{\prime}-775^{\prime}$

Type of core:

4 inch rotary

Core recovery:

$77.5^{\prime}$

Coring fluid: air

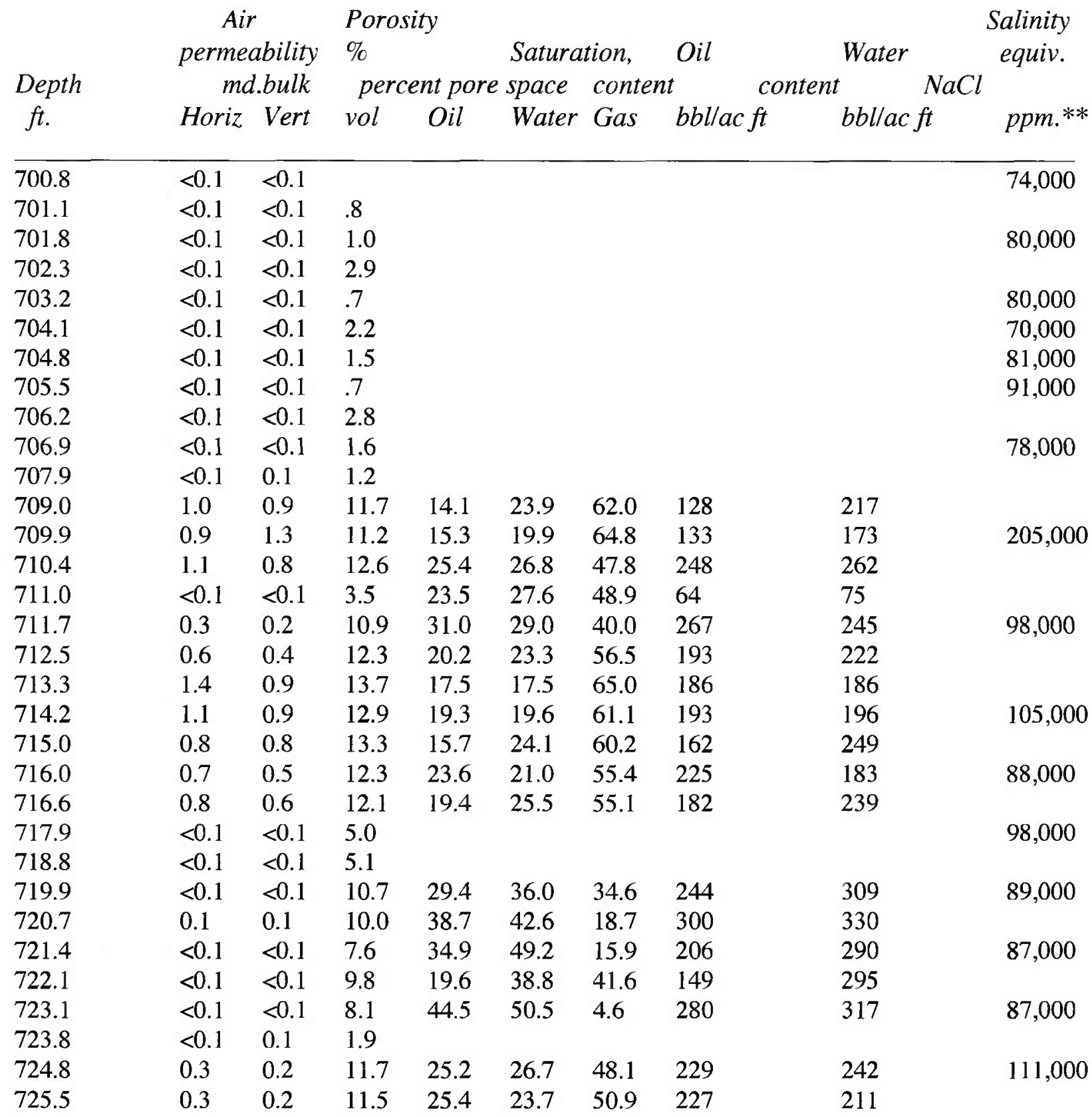




\begin{tabular}{|c|c|c|c|c|c|c|c|c|c|}
\hline 726.2 & $<0.1$ & 0.1 & 10.2 & 33.6 & 34.7 & 31.7 & 266 & 275 & 88,000 \\
\hline 727.1 & $<0.1$ & $<0.1$ & 10.8 & 33.6 & 24.1 & 42.3 & 282 & 203 & \\
\hline 728.2 & $<0.1$ & $<0.1$ & 2.2 & & & & & & \\
\hline 729.2 & $<0.1$ & 0.1 & 1.5 & & & & & & \\
\hline 730.2 & 0.4 & 0.1 & 11.7 & 28.7 & 28.1 & 43.2 & 261 & 255 & 92,000 \\
\hline 731.0 & 0.5 & 0.4 & 12.2 & 26.5 & 27.8 & 45.7 & 251 & 263 & \\
\hline 731.7 & 0.4 & 0.4 & 12.0 & 27.7 & 25.8 & 46.5 & 258 & 240 & 95,000 \\
\hline 732.8 & 0.3 & 0.3 & 11.4 & 30.6 & 23.2 & 46.2 & 271 & 205 & \\
\hline 733.5 & 0.3 & 0.2 & 11.7 & 31.6 & 28.5 & 39.9 & 287 & 259 & 101,000 \\
\hline 734.5 & 0.5 & 0.4 & 12.6 & 28.2 & 25.1 & 46.7 & 276 & 245 & \\
\hline 735.6 & $<0.1$ & $<0.1$ & 2.0 & & & & & & 192,000 \\
\hline 736.3 & 0.1 & $<0.1$ & 9.7 & 40.1 & 32.3 & 27.6 & 302 & 243 & \\
\hline 737.1 & 0.5 & 0.5 & 12.4 & 19.8 & 26.9 & 53.3 & 191 & 259 & 94,000 \\
\hline 738.0 & 0.7 & 0.6 & 12.4 & 27.3 & 26.3 & 46.4 & 263 & 253 & \\
\hline 738.7 & 0.2 & 0.1 & 10.1 & & & & & & \\
\hline 739.4 & 0.7 & 0.5 & 12.7 & 34.7 & 21.3 & 44.0 & 342 & 210 & 109,000 \\
\hline 739.8 & 0.5 & 0.5 & 12.6 & 27.7 & 28.0 & 44.3 & 271 & 274 & \\
\hline 740.8 & 0.5 & 0.5 & 12.1 & 25.8 & 27.3 & 46.9 & 242 & 256 & \\
\hline 741.8 & 0.6 & 0.5 & 12.5 & 28.3 & 28.0 & 43.7 & 274 & 272 & 100,000 \\
\hline 742.7 & $<0.1$ & 0.1 & 5.7 & 11.4 & 17.3 & 71.3 & 50 & 77 & \\
\hline 744.6 & $<0.1$ & 0.1 & 3.2 & 39.3 & 58.0 & 2.7 & 98 & 144 & 90,000 \\
\hline 745.3 & 0.1 & 0.1 & 9.9 & 33.9 & 38.4 & 27.7 & 260 & 295 & \\
\hline 746.3 & $<0.1$ & $<0.1$ & 9.3 & 35.4 & 49.0 & 15.6 & 255 & 354 & 90,000 \\
\hline 747.5 & 0.3 & $<0.1$ & 9.6 & 29.5 & 54.9 & 15.6 & 220 & 409 & \\
\hline 748.9 & 0.1 & $<0.1$ & 10.5 & 21.7 & 41.8 & 36.5 & 177 & 341 & 80,000 \\
\hline 749.8 & $<0.1$ & $<0.1$ & 4.6 & & & & & & \\
\hline 751.2 & $<0.1$ & $<0.1$ & 5.7 & & & & & & 98,000 \\
\hline 752.4 & $<0.1$ & $<0.1$ & 9.7 & & & & & & \\
\hline 753.6 & $<0.1$ & $<0.1$ & 0.8 & & & & & & 89,000 \\
\hline 756.8 & $<0.1$ & $<0.1$ & 1.1 & & & & & & \\
\hline 758.2 & $<0.1$ & $<0.1$ & 2.2 & & & & & & 86,000 \\
\hline 759.8 & $<0.1$ & $<0.1$ & 5.3 & 20.2 & 48.9 & 30.9 & 83 & 201 & \\
\hline 762.8 & $<0.1$ & $<0.1$ & 0.6 & & & & & & \\
\hline 766.0 & $<0.1$ & $<0.1$ & 1.0 & & & & & & \\
\hline 769.1 & $<0.1$ & $<0.1$ & 8.1 & 5.9 & 15.8 & 78.3 & 37 & 99 & \\
\hline 770.0 & 1.1 & $<0.1$ & 5.5 & 35.9 & 44.9 & 19.2 & 153 & 192 & \\
\hline 771.5 & $<0.1$ & $<0.1$ & 5.1 & 50.6 & 45.2 & 4.2 & 200 & 179 & \\
\hline 774.8 & $<0.1$ & $<0.1$ & 1.7 & & & & & & \\
\hline Ave values & 0.3 & 0.2 & 7.4 & 27.2 & 31.3 & 41.4 & 214 & 238 & 98,000 \\
\hline
\end{tabular}




\section{REPORT OF CRUDE PETROLEUM ANALYSIS}

Bureau of Mines Bartlesville Laboratory

F.C. Eaton well No. 3

$710-750$ feet

F.C. Eaton
Warren Quadrangle

Tiona Field

Glade sand
Item 55

Pennsylvania

Glade Township

\section{GENERAL CHARACTERISTICS}

Specific Gravity, 0.801

Sulphur, percent, 0.15

Saybolt Universal viscosity at $100 \mathrm{deg}$ F, $38 \mathrm{sec}$.
A.P.I. gravity, 45.2 degrees Color, N.P.A. No. 6

\section{DISTILLATION, BUREAU OF MINES HEMPEL METHOD}

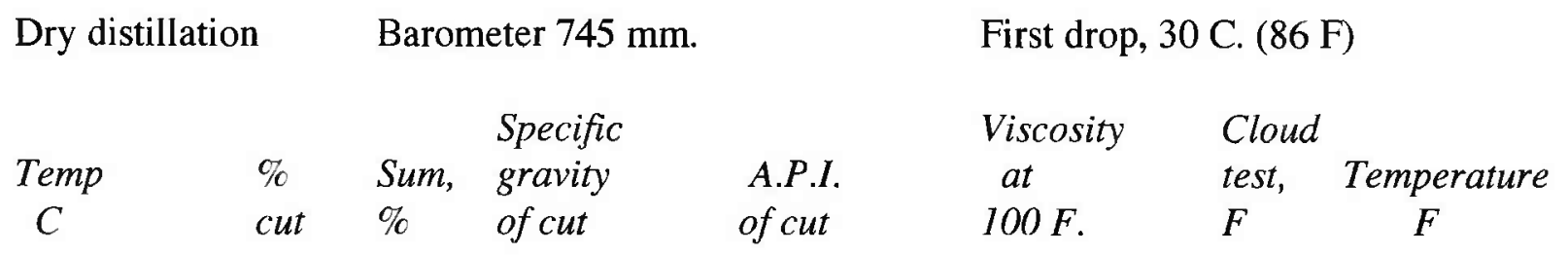

\begin{tabular}{|c|c|c|c|c|c|c|c|}
\hline$\overline{\mathrm{U}} \mathrm{p}$ to 50 & 1.4 & $1.4\}$ & & & & & Up to 122 \\
\hline $50-75$ & 2.6 & $4.0\}$ & 0.680 & 76.6 & & & $122-167$ \\
\hline $75-100$ & 5.3 & 9.3 & 0.698 & 71.2 & & & $167-212$ \\
\hline $100-125$ & 8.8 & 18.1 & 0.727 & 63.1 & & & $212-257$ \\
\hline $125-150$ & 7.9 & 26.0 & 0.746 & 58.2 & & & $257-302$ \\
\hline $150-175$ & 6.0 & 32.0 & 0.762 & 54.2 & & & $302-347$ \\
\hline $175-200$ & 6.3 & 38.3 & 0.776 & 50.9 & & & $347-392$ \\
\hline $200-225$ & 3.7 & 42.0 & 0.787 & 48.3 & & & $392-437$ \\
\hline $225-250$ & 5.6 & 47.6 & 0.797 & 46.0 & & & $437-482$ \\
\hline $250-275$ & 6.1 & 53.7 & 0.812 & 42.8 & & & $482-527$ \\
\hline \multicolumn{8}{|c|}{ Vacuum distillation at $40 \mathrm{~mm} . \mathrm{Hg}$} \\
\hline Up to 200 & 3.8 & 3.8 & 0.834 & 38.2 & 40 & 20 & Up to 392 \\
\hline $200-225$ & 5.1 & 8.9 & 0.839 & 37.2 & 46 & 35 & $392-437$ \\
\hline $225-250$ & 7.7 & 16.6 & .859 & 33.2 & 69 & 60 & $437-482$ \\
\hline $250-275$ & 5.7 & 23.3 & .864 & 32.3 & 87 & 70 & $482-527$ \\
\hline 275-300 & 5.7 & 29.0 & .879 & 29.3 & 120 & 90 & $527-572$ \\
\hline
\end{tabular}

Carbon residue of residuum, 1.6 percent; carbon residue of crude, 0.3 percent 


\section{APPROXIMATE SUMMARY}

\begin{tabular}{llllll} 
& $\%$ & Sp. Gr. & Deg API & Viscosity \\
Light Gasoline & 9.3 & 0.691 & 73.3 & \\
& & & & \\
\hline Total gasoline and Naptha & 38.3 & 0.736 & 60.8 & \\
Kerosene distillate & 15.4 & .801 & 45.2 & \\
Gas oil & 7.5 & .836 & 37.8 & \\
Nonviscous lubricating distillate 15.1 & $.842-.870$ & $36.6-31.1$ & $50-100$ & \\
Medium lubricating distillate & 6.4 & $.870-.886$ & $31.1-28.2$ & $100-200$ \\
Viscous lubricating distillate & - & - & - & Above 200 \\
Residuum & 16.3 & .898 & 26.1 & \\
Distillation loss & 1.0 & & & & \\
& & & & &
\end{tabular}

*Published in U.S. Bureau of Mines Report of Investigation 3385 


\section{Clarendon Formation}

\section{$\underline{\text { Introduction }}$}

The following information was taken from Mineral Resources Report M52 entitled "Oil and Gas Geology of the Warren Quadrangle, Pennsylvania." This document was published in 1965 by William S. Lytle of the Pennsylvania Geological Survey. Additional information was found in Mineral Resources Report M53 entitled "Oil and Gas Geology of the Youngsville Quadrangle, Pennsylvania." This document was published in 1964 by William G. McGlade of the Pennsylvania Geological Survey.

The Warren and Youngsville quadrangles are located in Warren County of northwestern Pennsylvania. The Warren quadrangle area is about 222 square miles. About one third is productive of oil and gas. The main producing sands are the Glade, Clarendon Stray, Clarendon, Gartland, and Cherry Grove of Upper Devonian age. The Youngsville quadrangle occupies the north-central portion of Warren County adjacent to the New York State Line. The first wells were drilled in this area as early as 1865 .

The term "sand" in this report refers to potentially productive zones of mostly sandstone with associated siltstones and shales. The terms "formation" or "rocks" in this report are more inclusive than the term "sand" and include transitional zones above and below the producing zone. The term "sandstone" refers only to beds of lithified sand.

\section{Historical Review}

The Tolles well was the first well drilled southeast of the Allegheny River in the Clarendon field. This well was drilled on January 13, 1878 at Bugsbee Mills, near Stoneham. Initial production from the Clarendon sand was 20 BOPD. Clarendon wells seldom had initial production rates of more than 25 BOPD.

Drilling in the Warren area continued at a fast rate from 1881 through 1895 . Since 1895 , drilling has been sporadic other than for waterflooding projects from the 1940's through the 1960's.

\section{Clarendon Formation}

The Clarendon Stray sand is between the base of the Glade rocks and the Clarendon sand. The Stray is around 40 feet thick. The top of the zone usually consists of about 20 feet of mediumgray shales and siltstones with rare fine-grained sandstone. When the siltstones and sandstones are well developed and productive of oil and gas in the lower 20 feet of the zone, the productive zone is called the Clarendon Stray sand. The Stray sand is a very fine- to fine-grained, light-gray sandstone. Near Big Bend the sand produces gas along the Allegheny River. Several drillers logs show the Clarendon Stray sand separated from the Clarendon sand by one-foot-thick fossiliferous cap rock. The top of the Clarendon rocks is the top of the zone of Clarendon Stray. This top averages 325 feet below the "Pink Rock."

In 1965, the Clarendon formation was the main oil-producing zone in the Warren quadrangle. The limits of the producing area were from the quadrangle south into the Sheffield quadrangle where this sand was one of the most important producers. As of 1963 , only one well was productive from the Clarendon section in the nearby Youngsville quadrangle. 
The true main body of Clarendon sand is more uniform than the other oil and gas producing sands in the Warren quadrangle. The sand is very fine-grained to pebbly and light-greenish-gray colored. The thickness varies from 0 to 50 feet, averaging 30 feet. The Clarendon contains very little interbedded shale. The productive area consists of one foot to an occasional eight feet of cap rock at the top, below which gas and then oil is found in the upper 10 feet. The sand is free from salt water. The Clarendon formation includes all sandy beds between the Glade formation above and a shale zone below which separates them from the underlying Speechley formation. When the Clarendon Stray sand is included with the Clarendon sand the interval from the base of the "Pink Rock" is 345 feet, whereas the interval from the base of the "Pink Rock" to the top of the true Clarendon sand is 365 feet.

The sandstone zones within the Clarendon formation are usually more erratic and thinner than the sandstone beds in the Glade formation. Several wells south of Youngsville show individual sandstone beds up to 6 feet thick. Cuttings from a well in the southeastern portion of the Youngsville quadrangle show about 11 feet of very fine-grained, silty, light-gray sandstone with traces of fine- to medium-size grains scattered throughout.

The Clarendon sand is best developed in the southeastern section of the Warren quadrangle where the main producing belt attains a width of over five miles. The Clarendon sand is frequently called "Third" and "Tiona" by the drillers. The base of the Clarendon is hard to determine. If the underlying Gartland is well developed, the top of the Gartland is considered to be the base of the Clarendon formation. When the base of the Clarendon rocks can be picked on the geophysical logs the lower unit of those rocks is called the zone of Clarendon sand. Approximately 40 feet thick, the zone contains the Clarendon sand at the top if developed, and shales and siltstones below.

The Clarendon formation is productive in the Morrison Run Pool, located about 4 miles southwest of Warren, Pennsylvania. This sandstone interval is usually very fine grained and light gray in color and averages about 22 feet in thickness. Sandstone beds in the Clarendon formation of the Youngsville quadrangle consist mainly of thin, very fine-grained and tight sandstone units in a predominant siltstone section.

Fields and Pools

Clarendon Field, Clarendon Pool

The Clarendon Pool, the largest pool in the Warren quadrangle, is 8 miles long and 4-1/2 miles wide. This pool trends north and south. Oil production comes from the Clarendon sand which is very fine- to fine-grained, light-gray sandstone containing some medium grains. The upper 4 to 5 feet of the sandstone unit is usually medium to very coarse grained and contains gas. The Clarendon Stray is hard to distinguish beween the Clarendon sand. The Stray lies immediately above the Clarendon sand and is separated from it at times by only a few feet of shale or by a foot of cap rock. 


\section{Clarendon Sand}

Porosities, permeablilities, saturations and oil contents

Clarendon Pool

Warren quadrangle, Warren County, Pennsylvania

\begin{tabular}{llllll} 
Pool Location & $\begin{array}{l}\text { Average } \\
\text { Porosity } \\
\%\end{array}$ & $\begin{array}{l}\text { Ave } \\
\text { Perm } \\
\text { md }\end{array}$ & $\begin{array}{l}\text { Ave Oil } \\
\text { Saturation } \\
\%\end{array}$ & $\begin{array}{l}\text { Ave Oil } \\
\text { Saturation } \\
\text { bbl/ac ft }\end{array}$ & $\begin{array}{l}\text { Oil cont } \\
\text { bbl/ac ft }\end{array}$ \\
\hline Northern End & 11.1 & 1.01 & & 184 & 10,200 \\
Northern End & 11.6 & 2.90 & & 220 & 6,250 \\
Middle & 12.4 & 2.08 & 27.8 & 295 & 8,201 \\
Middle & 14 & 11.80 & 25.3 & 282 & 7,954 \\
Middle & 12.03 & 1.04 & 21.1 & 197 & 5,527 \\
Southern End & 10.8 & 21.50 & & 278 & 7,370 \\
Southern End & 11 & 1.02 & & 233 & 5,670
\end{tabular}

Lithologic description of well cuttings

Well 013, lot 488, Morrison Run Field

D.W. Franchot

Pleasant Township, Warren County, Pa.

Elevation: 1594 Ground

Total Depth: 1391 feet

Description by W.R. Wagner

Depth (Feet)

Description of Strata

1219

1237-1246 Siltstone, 80\%, coarse-grained silt to fine-grained sand, 5GY7-8/1, in small part very slightly calcareous. Shale, $20 \%$, N6-N5, soft

1246-1248 Shale, N6, soft

1248

1248-1258 Sandstone, very fine-grained sand to coarse-grained silt, medium-light-greenishgray (5GY7/1) in part slightly micaceous, $10 \%$ slightly calcareous

1258-1260 Shale, N6, soft

1260-1265 Sandstone, similar to $1248-1258$ 
1265-1270 Sandstone as above 40\%, grading to silty sandstone. Shale, $60 \%$ soft, N6, soft 1270-1276 Siltstone, 70\% soft, coarse-grained, greenish-gray (5GY6/0.5) to N6, slightly to somewhat argillaceous. Sandstone, $30 \%$, very fine-grained, light-greenish-gray (5GY8/1-0.5), few pieces calcareous

1276-1294 Siltstone, 80\%, greenish-gray (5GY6-5/1-0.5), argillaceous to somewhat argillaceous, 5-10\% somewhat calcareous to calcareous, some brachiopod fragments 1289-94. Shale, 20\%, N5-N4

1294 Base of Clarendon rocks

\section{REPORT OF CRUDE PET ROLEUM ANALYSIS}

Bureau of Mines Bartlesville Laboratory

Clarendon Field

Clarendon Sand, Upper Devonian

981-1,006 feet
South Penn Oil Co. Pennsylvania

No. 38

Lot 106
Warren Co., Mead Twp.

Warren Quadrangle

\section{GENERAL CHARACTERISTICS}

Specific Gravity, 0.785

Sulphur, percent, less than 0.1

Saybolt Universal viscosity at $100 \mathrm{deg}$ F, $36 \mathrm{sec}$.
A.P.I. gravity, 48.8 degrees
Pour pt., deg F below 5

Color, N.P.A. No. $21 / 2$

\section{DISTILLATION, BUREAU OF MINES ROUTINE METHOD}

STAGE 1-Distillation at atmospheric pressure, $737 \mathrm{~mm}$. Hg

First drop, 27 C (81 F)

\begin{tabular}{|c|c|c|c|c|c|c|c|c|c|c|c|}
\hline \multirow[b]{2}{*}{$\begin{array}{c}\text { Fract. } \\
\text { No. }\end{array}$} & \multirow{2}{*}{\multicolumn{2}{|c|}{ Cut at }} & \multirow[b]{2}{*}{$\%$} & \multirow[b]{2}{*}{$\begin{array}{l}\text { Sum, } \\
\%\end{array}$} & \multirow[b]{2}{*}{$\begin{array}{l}\text { Sp. Gr.API } \\
60 / 60 F\end{array}$} & \multicolumn{2}{|l|}{ Deg } & \multicolumn{2}{|c|}{ Aniline } & \multirow{2}{*}{$\begin{array}{c}\text { S.U. } \\
\text { test } \\
100 \mathrm{~F}\end{array}$} & \multirow{2}{*}{$\begin{array}{c}\text { Cloud } \\
\text { F }\end{array}$} \\
\hline & & $F$ & & & & $60 F$ & point & $C$ & Visc & & \\
\hline
\end{tabular}

$\begin{array}{lllllllll}\overline{1} & 50 & 122 & 5.7 & 5.7 & 0.627 & 94.2 & - & - \\ 2 & 75 & 167 & 3.9 & 9.6 & .659 & 83.2 & 2.3 & 62.6 \\ 3 & 100 & 212 & 5.7 & 15.3 & .703 & 69.8 & 13 & 58.2 \\ 4 & 125 & 257 & 7.5 & 22.8 & .730 & 62.3 & 17 & 56.0 \\ 5 & 150 & 302 & 5.9 & 28.7 & .749 & 57.4 & 18 & 56.4 \\ 6 & 175 & 347 & 6.0 & 34.7 & .763 & 54.0 & 18 & 59.0 \\ 7 & 200 & 392 & 5.4 & 40.1 & .774 & 51.3 & 17 & 65.0 \\ 8 & 225 & 437 & 5.1 & 45.2 & .785 & 48.8 & 17 & 65.0 \\ 9 & 250 & 482 & 5.3 & 50.5 & .797 & 46.0 & 17 & 75.0 \\ 10 & 275 & 527 & 6.2 & 56.7 & .810 & 43.2 & 19 & 79.0\end{array}$


STAGE 2-Distillation continued at $40 \mathrm{~mm}$. $\mathrm{Hg}$

\begin{tabular}{|c|c|c|c|c|c|c|c|c|c|c|}
\hline 11 & 200 & 392 & 3.0 & 59.7 & 0.823 & 40.4 & 21 & 84.4 & 42 & 20 \\
\hline 12 & 225 & 437 & 5.1 & 64.8 & .832 & 38.6 & 21 & 88.2 & 45 & 35 \\
\hline 13 & 250 & 482 & 4.7 & 69.5 & .841 & 36.8 & 22 & & 55 & 55 \\
\hline 14 & 275 & 527 & 4.1 & 73.6 & .849 & 35.2 & 23 & & 73 & 70 \\
\hline 15 & 300 & 572 & 4.7 & 78.3 & .858 & 33.4 & 24 & & 115 & 80 \\
\hline \multicolumn{3}{|c|}{$\operatorname{Res}$} & 17.2 & 95.5 & .890 & 27.5 & & & & \\
\hline
\end{tabular}

Carbon residue of residuum, 0.9 percent; carbon residue of crude, 0.2 percent

\begin{tabular}{llllll}
\multicolumn{5}{c}{ APPROXIMATE SUMMARY } \\
& $\%$ & Sp. Gr. & Deg API & Viscosity \\
Light Gasoline & & & & \\
& 15.3 & 0.663 & 81.9 & \\
\hline Total gasoline and Naptha & 40.1 & 0.718 & 65.6 & \\
Kerosene distillate & 16.6 & .798 & 45.8 & \\
Gas oil & 8.0 & .828 & 39.4 & \\
Nonviscous lubricating distillate9.7 & $.836-.855$ & $37.8-34.0$ & $50-100$ & \\
Medium lubricating distillate & 3.9 & $.855-.863$ & $34.0-32.5$ & $100-200$ \\
Viscous lubricating distillate & - & - & - & Above 200 \\
Residuum & 17.2 & .890 & 27.5 & \\
Distillation loss & 4.5 & & & & \\
& & & & & \\
\hline
\end{tabular}

*Published by permission of Director U.S. Bureau of Mines 


\section{Gordon Formation}

\section{Introduction}

The following information was taken from Mineral Resources Report M54 entitled "Oil and Gas Geology of the Amity and Claysville Quadrangles, Pennsylvania." This document was published in 1967 by William G. McGlade of the Pennsylvania Geological Survey.

The Amity and Claysville 15-minute quadrangles are located in Washington County, Pennsylvania. These quadrangles include areas of once prolific oil and gas production. The principal oil- and gas-producing reservoirs in this area are located within the Conewango Group of the Upper Devonian Series. Five major sandstone units that can be found in this group are the Hundred-Foot (Gantz-Fifty Foot), Nineveh, Gordon, Fourth, and Fifth.

Drillers' well records were the main source of rock information in the studied area in addition to geophysical logs, well samples and cores. These records were evaluated for accuracy by comparing well geological data with composite sections of different portions of the studied area. The following lists a brief description of the "dependability index" assigned to the Gordon unit.

"Gordon zone.-In the Amity quadrangle, particularly in the eastern portion, the lower Nineveh may be confused with the upper part (Gordon Stray sandstone) of the Gordon zone and some difficulty may be encountered in separating the base of the Gordon from the underlying Fourth sandstone. In the western portion of the Amity quadrangle and in parts of the eastern portion of the Claysville quadrangle the Gordon Stray-Gordon separation is usually easily defined. In a large part of the Claysville quadrangle, the Gordon is a simple, undifferentiated unit while in a large part of the Amity quadrangle, the Gordon zone is multi-layered and very complex. The boundaries of the Gordon, particularly the top, are usually dependable but the Gordon Stray-Gordon separation is not possible when the total zone is complex."

\section{Historical Review}

In 1885, oil was discovered in the Gordon sand of the giant Washington-Taylorstown field. This sandstone reservoir would be the most prolific oil producer in the area. In 1886, a Gordon sandstone discovery of 100-170 barrels of oil per day was completed about one mile southeast of Hill Church. Intense activity followed this discovery. By 1965, very little exploration or development drilling had been carried on for many years.

\section{Gordon Formation}

The Gordon Zone is probably more complex than any other unit in the Upper Devonian of the Amity and Claysville quadrangles. In the southeastern portion of the Amity quadrangle this formation averages 90 feet thick and is a multi-layered sequence of sandstone beds. Further west where the Gordon zone is oil productive the zone is greater than 60 feet thick. The oil-producing interval is usually located at the base of the zone. Further west the sandstone beds thin and finally shale out west of the Donegal gas storage field. This storage field is located along the western edge of the Claysville quadrangle. In portions of the northeastern and central eastern portions of the Amity quadrangle the Gordon zone has usually been logged as a one or two sandstone unit. Gamma ray logs show that the zone is much thicker and very complex compared 
to drillers' log data. In this area the Gordon may be silty or very fine grained and therefore not recorded by the driller.

Ten to thirty feet of red and dark gray shale separate the Gordon formation from the overlying Niveneh zone. In the Claysville quadrangle and the western portion of the Amity quadrangle, this boundary is usually recognized. In the eastern portion of the Amity quadrangle where the Gordon zone is muli-layered, the boundary may be difficult to difficult to determine without geophysical well log data.

The sandstones of the Gordon zone are white to light gray, very fine to coarse grained, with conglomerate and coarse-grained sandstones in thin beds. The roundness of grains increases with grain size. The coarser beds exhibit poorer sorting. Gray shales predominate throughout the interbedded shale sequence. However, the shales may be red near the top.

Most of the oil production is from the basal Gordon sandstone with minor amounts coming from the upper Gordon Stray sandstone unit. Gas production

Analyses of four cores

WASHINGTON OIL COMPANY

Jas. McMannis No. 9

Washington Co., PA

GORDON SANDS

$2471 \mathrm{ft}-2500.7 \mathrm{ft}$

Sample No. Depth in feet Permeability Md. Percent Porosity

\section{GORDON STRAY}

$\begin{array}{llll}X-1 & 2471.2 & .23 & 11.82 \\ X-2 & 2473.3 & .68 & 13.21 \\ X-3 & 2474.4 & .44 & 12.63 \\ X-4 & 2475.4 & \text { Impermeable } & 11.54 \\ X-5 & 2478 & \text { Impermeable } & 2.63\end{array}$

GORDON SAND

X-6

S-1

S-2

$\mathrm{X}-7$

S-3
2492.2

2492.5

2493.0

2493.9

2494.7
2.47

6.3

3.62

20.75

26.83
13.19

17.37

15.24

18.08

19.17 


$\begin{array}{llll}\text { X-8 } & 2495.3 & 37.04 & 21.59 \\ \text { S-4 } & 2495.9 & 108.32 & 20.59 \\ \text { S-5 } & 2496.5 & 0.16 & 17.25 \\ \text { X-9 } & 2497.0 & 182.1 & 22.50 \\ \text { S-6 } & 2497.5 & 221.92 & 25.91 \\ \text { S-7 } & 2498.0 & 239.69 & 20.00 \\ \text { X-10 } & 2498.5 & 188.85 & 23.44 \\ \text { S-8 } & 2499.0 & 284.54 & 28.29 \\ \text { S-9 } & 2499.5 & 166.65 & 23.94\end{array}$

WASHINGTON OIL COMPANY

Jas. Hodgins, Sr. No. 13

Blaine Township

Washington Co., PA

GORDON SAND

Saturation

Depth

feet

2551.0

2551.5

2552.7

2553.4

2553.8

2554.5

2555.0

2555.5

2556.0

2557.3
Perm,

md

4.8

16.4

314.2

328.2

285.3

290.9

61.7

106.8

33.1

4.2
Porosity, percent

11.72

11.07

26.37

27.19

26.80

26.41

21.81

23.17

17.38

9.68 percent pore volume

Oil Water

Oil

content $\mathrm{bbl} / \mathrm{ac}-\mathrm{ft}$
Chloride content of water in cores, ppm

Note: Analyzed by United States Bureau of Mines 


\section{WASHINGTON OIL COMPANY \\ Lemon Carson No. 10 \\ Taylorstown Field \\ Washington Co., PA GORDON SAND}

\begin{tabular}{|c|c|c|c|c|c|c|}
\hline $\begin{array}{l}\text { Depth } \\
\text { feet }\end{array}$ & $\begin{array}{l}\text { Perm, } \\
\text { md }\end{array}$ & $\begin{array}{l}\text { Porosity, } \\
\text { percent }\end{array}$ & \multicolumn{2}{|c|}{$\begin{array}{c}\text { Saturation } \\
\text { percent pore volume }\end{array}$} & $\begin{array}{l}\text { Oil } \\
\text { content } \\
\text { bbl/ac-ft }\end{array}$ & $\begin{array}{l}\text { Chloride } \\
\text { content of } \\
\text { water in cores, } \\
\text { ppm }\end{array}$ \\
\hline 2548.0 & 0.7 & 5.99 & 0.0 & 99.67 & 0 & \\
\hline 2548.7 & 195.4 & 25.15 & 5.96 & 92.07 & 116 & 16461 \\
\hline 2549.5 & 242.0 & 26.66 & 7.67 & 80.33 & 159 & \\
\hline 2550.5 & 14.0 & 24.22 & 13.07 & 78.77 & 245 & 12637 \\
\hline 2551.5 & 2.4 & 24.69 & 21.95 & 68.04 & 420 & \\
\hline 2552.0 & 1.2 & 27.11 & 20.67 & 69.33 & 435 & 37376 \\
\hline 2552.5 & 276.5 & 25.99 & 19.84 & 70.71 & 400 & \\
\hline 2553.0 & 297.6 & 26.67 & 17.76 & 67.06 & 367 & 30299 \\
\hline \multicolumn{6}{|c|}{ Chloride content of water produced with oil, Lemon Carson No. 2} & 37762 \\
\hline
\end{tabular}

Note: Analyzed by United States Bureau of Mines 
CHARLES E. YOUNG AND ASSOC.

No. 1 J.L. Kenamond

Washington Co., PA

GORDON SAND

\begin{tabular}{|c|c|c|c|c|c|c|}
\hline $\begin{array}{l}\text { Depth } \\
\text { feet }\end{array}$ & $\begin{array}{l}\text { Perm, } \\
\text { md }\end{array}$ & $\begin{array}{l}\text { Porosity, } \\
\text { percent }\end{array}$ & $\begin{array}{l}\text { Sat } \\
\text { percen } \\
\text { Oil }\end{array}$ & $\frac{\text { volume }}{\text { Water }}$ & $\begin{array}{l}\text { Oil } \\
\text { content } \\
\text { bbl/ac-ft }\end{array}$ & $\begin{array}{l}\text { Chloride } \\
\text { content of } \\
\text { water in cores, } \\
\text { ppm }\end{array}$ \\
\hline 2527.7 & 0.3 & 2.49 & 25.62 & 72.02 & 50 & \\
\hline 2428.4 & 0.9 & 2.28 & & & & \\
\hline 2432.5 & 0.3 & 3.62 & 15.89 & 80.41 & 45 & \\
\hline 2443.1 & 1.5 & 4.47 & & & & \\
\hline 2448.1 & 0.4 & 3.54 & & & & \\
\hline 2450.0 & 1.0 & 7.87 & & & & \\
\hline 2452.7 & 0.7 & 4.59 & & & & \\
\hline 2459.5 & 9.6 & 6.57 & & & & \\
\hline 2462.1 & 11.6 & 11.21 & & & & \\
\hline 2462.7 & 3.9 & 11.19 & & & & \\
\hline 2462.8 & 96.0 & 10.11 & & & & \\
\hline $2465.7 \mathrm{a}$ & 0.9 & 8.14 & 25.59 & 14.22 & 162 & \\
\hline $2468.3 b$ & 2.5 & 11.69 & 24.86 & 27.16 & 226 & 111916 \\
\hline 2469.6 & 1.9 & 10.31 & & & & \\
\hline $2470.4 a$ & 0.8 & 9.24 & 17.16 & 32.60 & 123 & \\
\hline 2472.2 & 1.1 & 8.48 & & & & \\
\hline $2473.1 b$ & 0.9 & 9.16 & 14.47 & 45.08 & 103 & \\
\hline 2475.6 & 3.2 & 7.72 & & & & \\
\hline 2477.1 & 1.0 & 8.37 & 25.80 & 41.95 & 168 & 129271 \\
\hline 2478.5 & 71.5 & 7.82 & 22.60 & 13.09 & 137 & 21815 \\
\hline 2479.5 & 1.1 & 7.68 & & & & \\
\hline 2484.2 & 0.4 & 4.52 & & & & \\
\hline 2486.0 & 0.4 & 5.55 & & & & \\
\hline
\end{tabular}

a Sample was not placed in air-tight container until 36 hours after its removal from core barrel.

b Sample was not placed in air-tight container until 12 hours after its removal from core barrel.

Note: Analyzed by United States Bureau of Mines 
Lithologic description of cores

Washington Oil Company No. 13 Jas. Hodgins, Sr.

Blaine Township, Washington County, $\mathrm{Pa}$.

Description by R.C. Stephenson

\section{DESCRIPTION OF CORES}

Depth

ft.

2545

in. $f$

in

25463

$2546 \quad 3$

$2547 \quad 3$

2547

3

25521

2552
Core No. 1

Cored 1 foot 3 inches, recovered 1 foot.

Recovered about 1 foot of dark gray shale showing pronounced bedding plane fracture. No sand. Drilled hard. No samples for analysis.

Core No. 2

Cored 1 foot, recovered 1 foot.

Eight inches broken material, somewhat coarser than cuttings, composed of dark-gray shale and greenish-gray, fine sand.

Fout inches sandstone, small, broken biscuits, light greenishgray, fine, hard, tight, subangular grains, no petroliferous odor. Drilled hard. Not sampled for analysis.

Core No. 3

Cored 4 feet 10 inches, recovered 1 foot.

Four inches broken material, somew hat coarser than cuttings, composed of dark gray shale and fine gray sand, as above, with shale fragments predominating. No biscuits. Separated from sand in lower part of core barrel by broken clayey shale packed in the barrel.

Eight inches sandstone, light gray, fine, fairly hard, with random distribution of small, well-rounded pebbles. A few small biscuits and much pulverized sand. Quite strong petroliferous odor. Samples 1 and 2 taken from this zone for analysis.

At 2548 feet was a marked drilling break, probably marking the top of the Gordon Sand.

\section{Core No. 4}

Cored 3 feet 3 inches, recovered 3 feet 3 inches.

Three feet, three inches sandstone, light gray with greenish cast, fine at top grading to medium fine below, with some pebbles in the sand near the top, slightly micaceous, becoming more soft and 
friable in the lower two feet. Strong petroliferous odor, with small amount of light green oil along some fresh fractures. Samples $3,4,5,6$ and 7 come from this core. All of this interval drilled easily.

Depth

$f$.

$\begin{array}{llll}2555 & 4 & 2557 & 10\end{array}$

Steel line measure at 2560 feet
Core No. 5

Cored 2 feet 6 inches, recovered 2 feet 2 inches.

One foot, one inch sandstone, light greenish-gray, fine, fairly soft, somewhat friable, increasing hardness toward base where there are some random pebbles scattered through the sand. Strong odor and traces of light green oil decrease downward.

Nine inches, shale, dark gray, very hard.

Four inches sandstone, light gray, very fine, hard, tight, with no apparent odor on fresh fracture. Sample 10 from this interval.

Core No. 6

$\begin{array}{lllll}2557 & 10 & 2559 & 10 & \text { Cored } 2 \text { feet, recovered } 3 \text { inches. }\end{array}$

Three inches sandstone, medium gray, very fine, hard, tight, with some very thin dark gray shaly streaks.

Description of Gordon samples from two wells

Gulf Oil Company No. 1 Lone Pine Unit

Elevation (KB) 1244 feet

Description by W.G. McGlade

Depth (Feet)

Sample Description

$2750-2800$

2750-2801

2750-2802

2750-2803

2750-2804

2750-2805

2750-2806

2750-2807

$2750-2808$
Siltstone: light gray

Siltstone: red and Shale: dark gray TOP OF GORDON SANDSTONE

Sandstone: very fine to coarse grained, mostly fine grained, light gray

Shale: dark greenish-gray and Sandstone: as above

Sandstone: very fine to medium grained, light gray

Shale: silty, mostly red, and medium greenish-gray

Sandstone: very fine to fine grained, silty, light reddish-gray

Sandstone: very fine grained, light greenish-gray BASE OF GORDON

SANDSTONE

Shale: silty, light gray 
Charles E. Young and others, Harry Hatfield No. 1

North Strabane Township, Washington County, $\mathrm{Pa}$.

Completed January, 1946

Elevation 1343 feet

Description by C.R. Fettke

(modified by W.G. McGlade)

Depth (Feet)

Sample Description

2650-2668 Shale, dark gray, with some interbedded very fine to fine-grained light gray sandstone and a little purplish-red silty shale

2668 Top of Gordon Sand

2668-2690 Sandstone, fine-grained, light gray, with considerable interbedded gray shale

2690-2700 Sandstone, very fine-grained, light gray, with a great deal of interbedded gray shale

$2700 \quad$ Bottom of Gordon sand

2700-2742 Shale, dark gray, with a little interbedded very fine-grained, light gray sandstone

Analysis of oil sample from the Gordon sand in the Washington Field, Washington County, Pennsylvania. Data from the "Report of Investigations, Analyses of Crude Oils from Some Fields of Pennsylvania and New York" by E.C. Lane and W.L. Gardon, January 1938, p. 58-62:

Sample 23390

W.W. Moore well no. 2

2,631-2,637 feet

Manufacturers Light \& Heat Co.
Item 50

Pennsylvania

Washington County

N. Franklin Township

Washington Field

Gordon Sand

GENERAL CHARACTERISTICS 
Specific Gravity, 0.800

Sulphur percent, less than 0.10

Saybolt Universal viscosity at $100 \mathrm{deg} F, 44 \mathrm{sec}$.
A.P.I. gravity, 45.4 degrees

Color, dark green

APPROXIMATE SUMMARY

\begin{tabular}{llllll} 
& $\%$ & Sp. Gr. & Deg API & Viscosity \\
Light Gasoline & 0.8 & & & \\
\hline Total gasoline and Naptha & 24.3 & 0.748 & 57.7 & \\
Kerosene distillate & 23.5 & 0.788 & 48.1 & \\
Gas oil & 10.2 & 0.827 & 39.6 & \\
Nonviscous lubricating distillate 4.4 & $0.832-0.855$ & $38.6-34.0$ & $50-100$ & \\
$\begin{array}{l}\text { Medium lubricating distillate } \\
\text { Viscous lubricating distillate }\end{array}$ & 3.7 & $0.855-0.859$ & $34.0-33.2$ & $100-200$ \\
$\begin{array}{l}\text { Residuum } \\
\text { Distillation loss }\end{array}$ & 21.7 & 0.889 & 27.7 & Above 200
\end{tabular}




\section{Akron Dolomite (Bass Islands) Trend}

Introduction

The "Bass Islands Trend" is a popular name for a long (38-40 mile) narrow, (1-1/2 miles or less wide) discontinuous belt of productive oil and gas wells in portions of Chautauqua, Cattaraugus and Erie Counties, New York. The largest part extends from east of the village of Sinclairville southwest to the village of Clymer, crossing Chautauqua Lake at the narrows.

The productive formation is usually the Onondaga Limestone and occasionally the underlying Akron Dolostone. These formations are approximately 1000 feet above the Medina Group. The Onondaga is present at the surface in the Buffalo area.

The name "Bass Islands" is a misnomer that has been used by drillers in the trend. Bass Islands is a Canadian and Ohio term for the Akron-Bertie Group of New York. This group is composed of rocks just below the Onondaga Limestone.

\section{History}

National Fuel's Zoar field was the first field in the Bass Islands Trend which produced during the late 1800 's until 1916 when it was converted to storage. No other Onondaga Akron production was encountered until 1963 when Wolf's Head Refining drilled a well near Ellery Center just northeast of Chautauqua Lake. Drilled as a deep test, the well penetrated through a faulted Onondaga section and some oil and gas production was reported in a zone below the unconformity separating the middle Devonian from Upper Silurian. However, the well was considered non-commercial and was plugged. In early 1981, a natural flow of gas was encountered in the Onondaga Limestone in a well at Gerry, New York. The operator overlooked the importance of the gas in the Onondaga and simply completed the Medina.

In February 1981, an operator based in Hamburg, New York drilled a well in the southwestern corner of Chautauqua County. This well was originally scheduled to be a 4500 feet Medina test well. However, the well blew out and flowed oil from a depth of 2885 feet. Before the well was brought under control, several hundred barrels of high gravity crude oil were reported to have flowed to the surface. Amazingly, this well was the first flowing oil well in the state of New York in nearly forty years from a zone not commonly recognized as productive for hydrocarbons. Numerous discoveries followed, dispelling the idea that the first was an isolated occurrence.

Bass Islands Trend In early 1980, repetition of section in Gamma Ray logs was noticed in several Medina wells in Chautauqua County, New York. The repeated section was generally part of the interval from above the top of the Devonian Onondaga Limestone to the mid point of the Silurian Salina. Reverse faulting caused this structural complication. Wells with repeated sections often had gas and/or oil shows at the fault break. These shows were generally considered uneconomic.

Further drilling into this trend led to three conclusions. The faults are enechelon and genetically related. These faults traverse the county laterally from northeast to southwest. The faults are located in a nartow fairway about two miles wide. 
Art Van Tyne of the New York State Oil \& Gas Research Office mapped and showed the same trend in several 1980 publications. Mr. Van Tyne projected two parallel reverse faults that traversed Chautauqua County and continued into adjacent Catteraugus and Erie Counties. Isopach maps of the interval from the top of the Onondaga to the base of the Packer Shell across Chautauqua County show thickening in the trend area.

The Harrington \#1 well in Ellery Township was drilled by Wolfshead Refining in 1963 in the trend. This well was drilled as a deep test, but was unsuccessful. A small amount of oil and gas production was reported in a zone below the unconformity separating the Middle Devonian from Upper Silurian. 


\section{APPENDIX D. DEVELOPMENT OF A NUMERICAL SOLVER FOR WAVE PROPAGATION IN POROUS MEDIA}

\section{Previous work on Wave Propagation in Porous Media}

The state of knowledge in this field was summarized by Corapcioglu [1990]. He pointed out that the continuum approach to this problem stems from the principles of mass, momentum, and energy conservation. The rock matrix is considered as a continuous rigid or elastic medium that is saturated by single or a multiphase fluid. The fluid phase is considered to exist in a continuous manner in the interconnected solid pores. The coupling of the conserved variables between the fluid and the solid is achieved by employing constitutive relationships (i.e., and equation of state of the fluid, and a stress, strain relationship in the solid). The necessary constants for these constitutive relationships are commonly determined in laboratory studies |Biot and Willis, 1957| although theoretical developments have been reported as well [Mindlin, 1957]. A mathematically elegant treatment of porous media continuum was developed by Bear and Bachmat [1990]. In this approach the system of partial differential equations governing the process is developed by considering a Representative Elementary Volume (REV) and proceeding from processes that take place microscopically to achieve a macroscopic description of the response of porous media to dynamic pressure loading.

\section{Mathematical Formulation}

Systems of hyperbolic PDEs are customerly formulated in the form

$$
\frac{\partial U}{\partial t}+\frac{\partial F(U)}{\partial x}=Q(U)
$$

where, $\mathrm{U}$ is the conserved vector, $\mathrm{F}$ is the flux vector and $\mathrm{Q}$ is the forcing function. These mathematically convenient definition take on special forms. Following the development of Bear and Bachmat |1990|, and the subsequent development of Levy et. al [ 1995], it can be shown that the conserved variables my be defined as

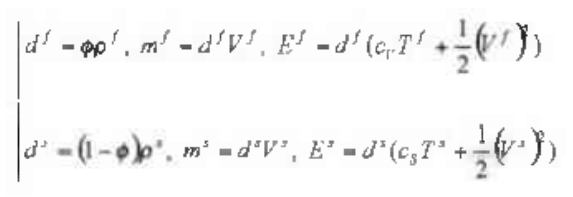


Where $\phi$ is the porosity of the solid matrix, $c_{V}$ is the heat capacity of the fluid at constant volume, and $C_{\mathrm{r}}$ is the heat expansion coefficient of the rock. By noting that both momentum and energy have kinetic and internal components and following the averaging method described by Bear and Bachmat [1990], it can be shown that the flux vector is given by

$$
F=\left|\begin{array}{l}
m^{\prime} \\
m^{\prime} \\
\frac{\left(m^{\prime}\right)}{d^{\prime}}+\phi \xi P \\
\frac{\left(m^{\prime}\right)}{d^{\prime}}+(1-\phi \xi) P-\sigma^{\prime} \\
\frac{m^{\prime}}{d^{\prime}}\left[E^{f}+\phi \xi p\right] \\
\frac{m^{2}}{d^{\prime}}\left[E^{x}+(1-\phi \xi) P\right]
\end{array}\right|
$$

where $\sigma$ is the stress in the solid, $P$ is the pressure of the fluid, and $\xi$ is a coefficient indicating the degree of tortuosity of the rock. This term can be better understood as the ratio length of a porous specimen to distance traveled by a fluid particle through a the interconnected pores of the same specimen. A good discussion on this term has been provided by Carman.

Momentum and energy transfer between the two sources occurs through friction loss. Following the derivation, it was found that

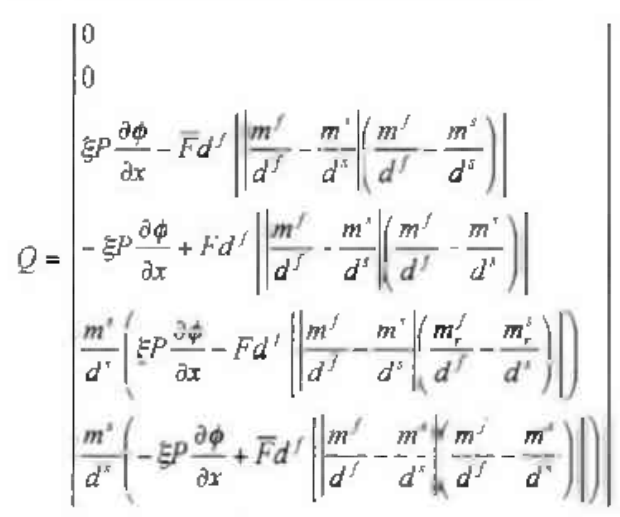

Where $\bar{F}$ is a friction constant, sometimes referred to as the Forchheimer term [Levy, 1995].

The system (D3) consists of six equations in six conserved unknowns which is a mathematically close system. However constitutive relationships are needed to relate the non-conserved variable to the conserved variables. The following relationships were used for this purpose

$$
p=\frac{(y(P, T)-1)}{\phi}\left|E^{f}-\frac{\left(m^{j}\right)}{2 d^{f}}\right|
$$


$\sigma^{x}=E_{e}\left(1-\frac{d^{s}}{\rho^{s}}\right)-E_{T} C \cdot\left(T^{s}-T^{s 0}\right)$

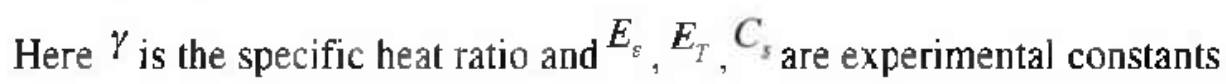

\section{Numerical Solver}

A high resolution Total Variation Diminishing (TVD) hyperbolic equation system solver was used. Numerically, the system of transport equations can be solved using the following upwind scheme:

$$
U_{j}^{n+1}=U_{j}^{n}-\frac{\Delta t}{\Delta x}\left(\bar{F}_{j=1}-\bar{F}_{j+1}\right)+\Delta l Q_{j}
$$

The modified numerical flux is given by:

$$
\begin{aligned}
& \bar{F}_{j+1}=\frac{1}{2}\left|F\left(U_{j}^{*}\right)+F\left(U_{j+1}^{*}\right)-\frac{\Delta x}{\Delta t} \sum_{i=1}^{6} \beta_{j}^{\ell} \cdot \mathbf{R}{ }_{j+1 / 2}^{k}\right| \\
& \beta_{j+1 / 2}^{k}=\Psi^{k}\left(v_{j+1 / 2}^{k}+\gamma_{j+1 / 2}^{\alpha}\right) k_{j+1 / 2}^{k}-\left(g_{j}^{k}+g_{j+1}^{k}\right) \\
& v_{j+1 / 2}^{k}=\frac{\Delta t}{\Delta t} a^{k}\left(U_{j+1}^{n}\right) \\
& \gamma_{j+1 / 2}^{*}- \begin{cases}\left(g_{j+1}^{k}-g_{j+1}^{k}\right) \alpha_{j+1 / 2}^{k}, & \alpha_{j+1 / 2}^{k}=0 \\
0, & \alpha_{j+1 / 2}^{k}=0\end{cases} \\
& g_{j}^{k}=\operatorname{sgn}\left(\tilde{g}_{j+1 / 2}^{k}\right) \max \left[0, \min \left(\tilde{g}_{j+1 / 2}^{k}, \hat{g}_{j-1 / 2}^{k} \operatorname{sgn}\left(\widetilde{g}_{j+1 / 2}^{k}\right)\right)\right. \\
& \Psi(x)= \begin{cases}x^{2} / 4 \xi+\xi, & |x|<2 \xi \\
|x| & |x| \geq 2 \xi\end{cases} \\
& \xi=0.1
\end{aligned}
$$

The rest of the values in the previous equation are provided in details elsewhere |Levy et al., $1995]$.

\section{Numerical Solver Validation}

The Model was first tested for the single phase gas using a the following Riemann problem:

$$
U(x)=\left\{\begin{array}{l}
U_{L}, x \leq \frac{L}{2} \\
U_{R}, x>\frac{L}{2}
\end{array}\right.
$$




$$
U_{L}=\left[\begin{array}{c}
11.7683 \\
0.00000 \\
0.00000 \\
0.00000 \\
2533125 \\
0.00000
\end{array}\right], U_{R}=\left|\begin{array}{c}
1.17683 \\
0.00000 \\
0.00000 \\
0.00000 \\
253312.5 \\
0.00000
\end{array}\right|
$$

Here an excellent agreement with the exact solution presented by Levy et. al [1996| is evident from Figures D1 through D3.

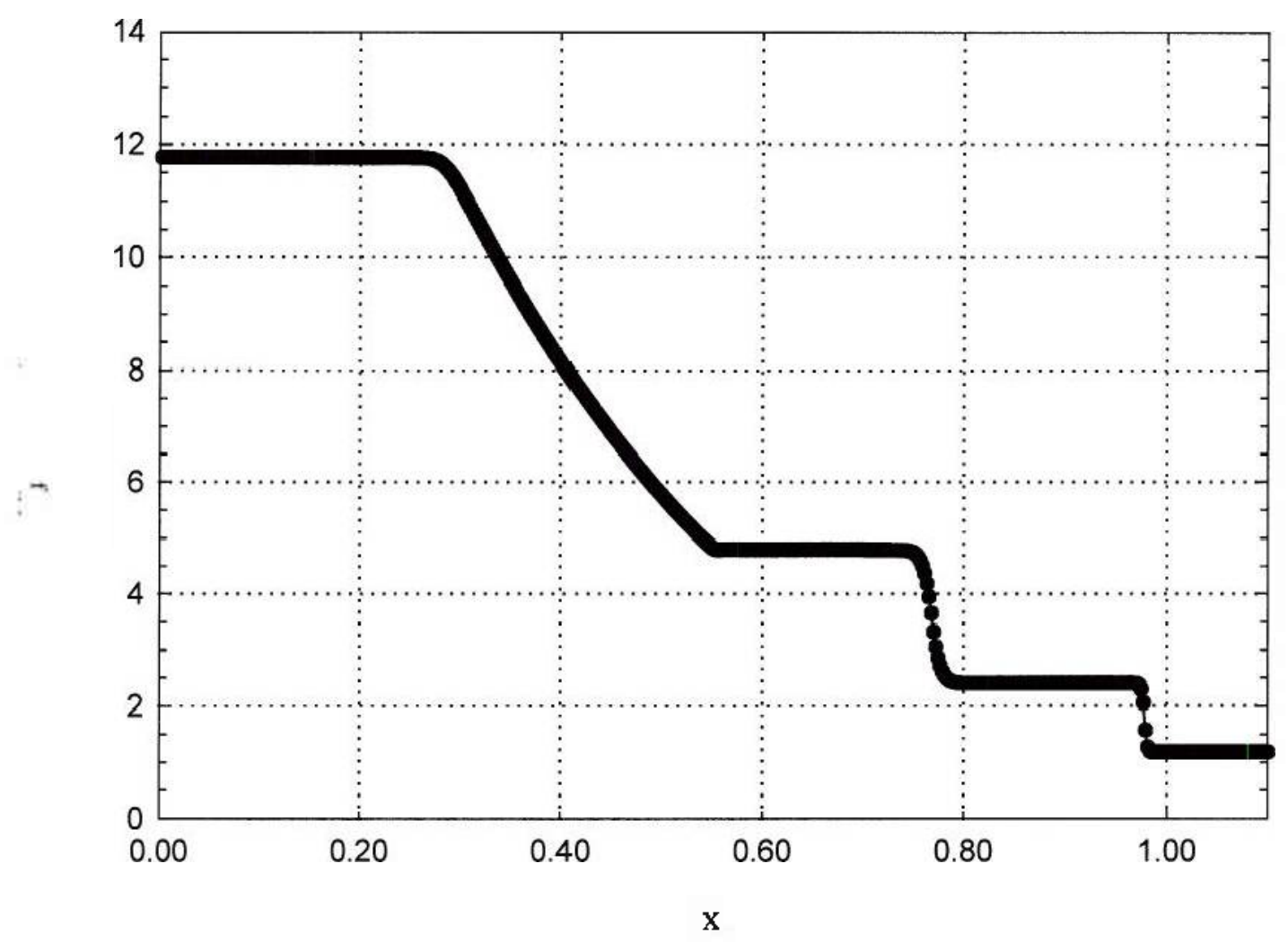

Figure D1: Comparison of exact and numerical solutions for the density term. 


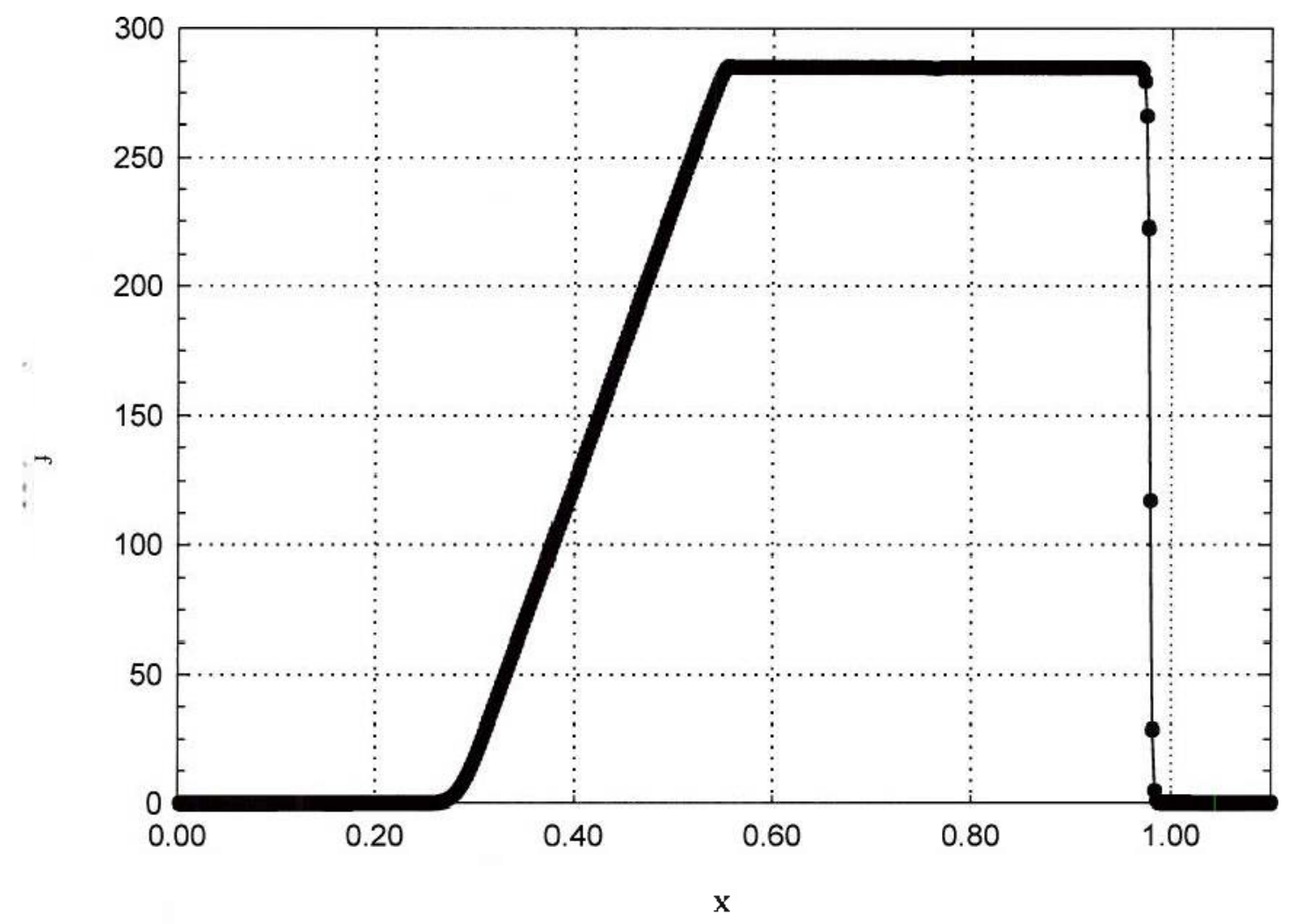

Figure D2: Comparison of exact and numerical solutions for the density term.

\section{Validation of Model with Shock Tube Test}

The model was tested using the shock tube problem presented by Levy et al. [1996]. The shock tube experiment was conducted using a $40 \mathrm{~cm}$ tube. The following figures show the pressure profiles in the tube at particular time. The results are compared with the those reported by Levy et al. 


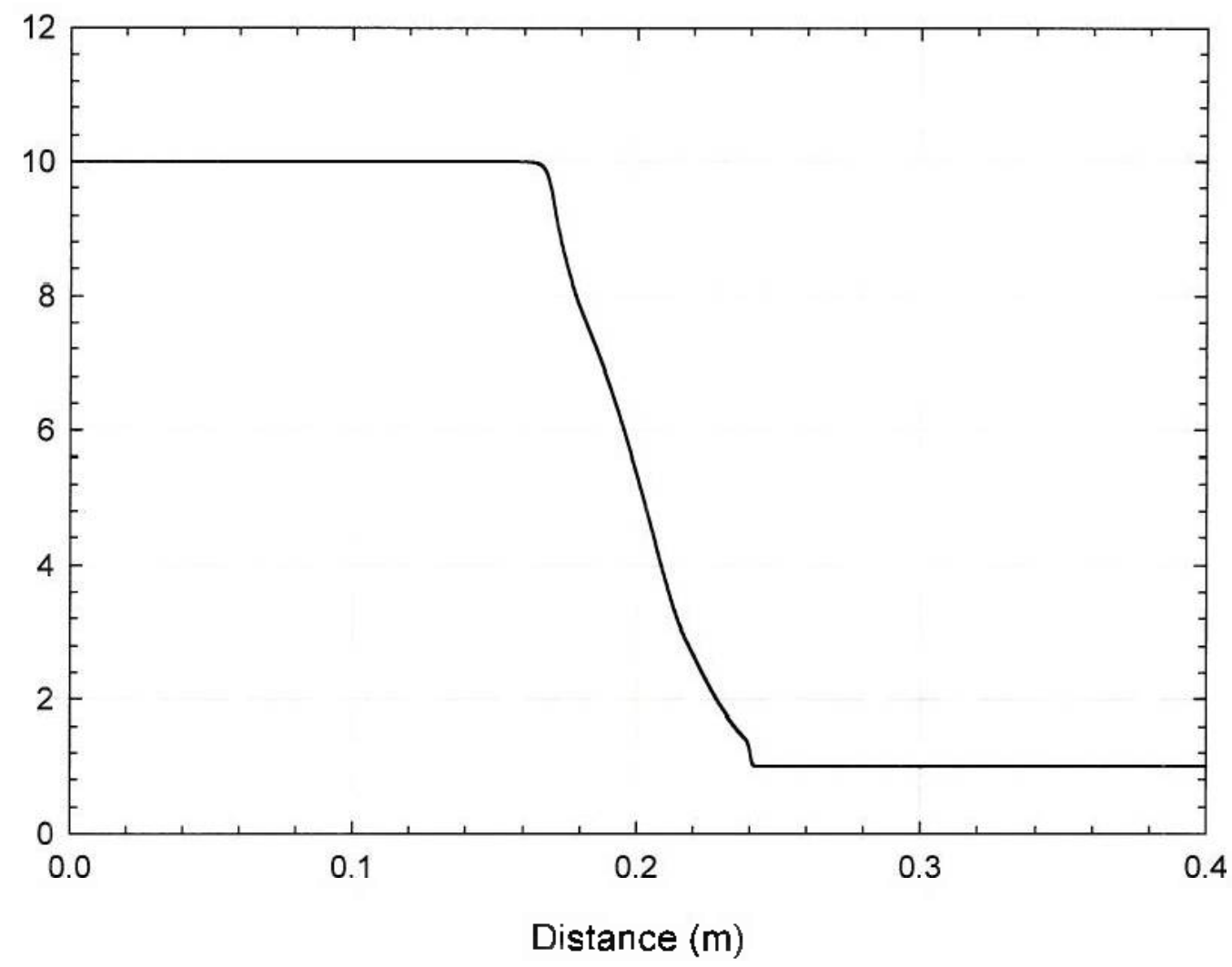

Figure D3: Pressure profile in a $40 \mathrm{~cm}$ porous sample after $130 \mathrm{~ms}$ from shock initiation. 




Figure D3: Fluid density profile in a $40 \mathrm{~cm}$ porous sample after $130 \mathrm{~ms}$ from shock initiation. 


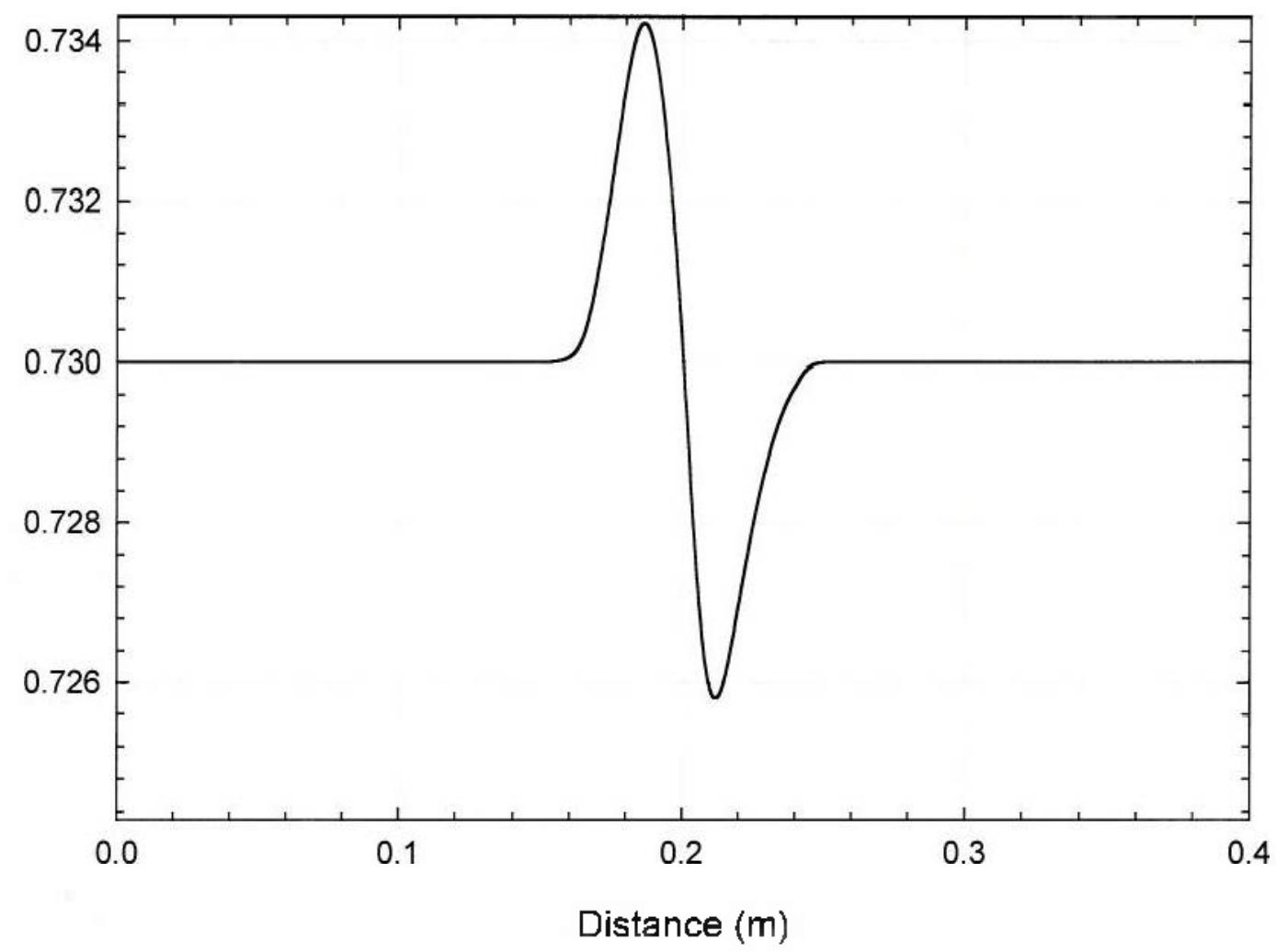

Figure D4: Porosity profile in a $40 \mathrm{~cm}$ sample $130 \mathrm{~ms}$ after shock initiation. 


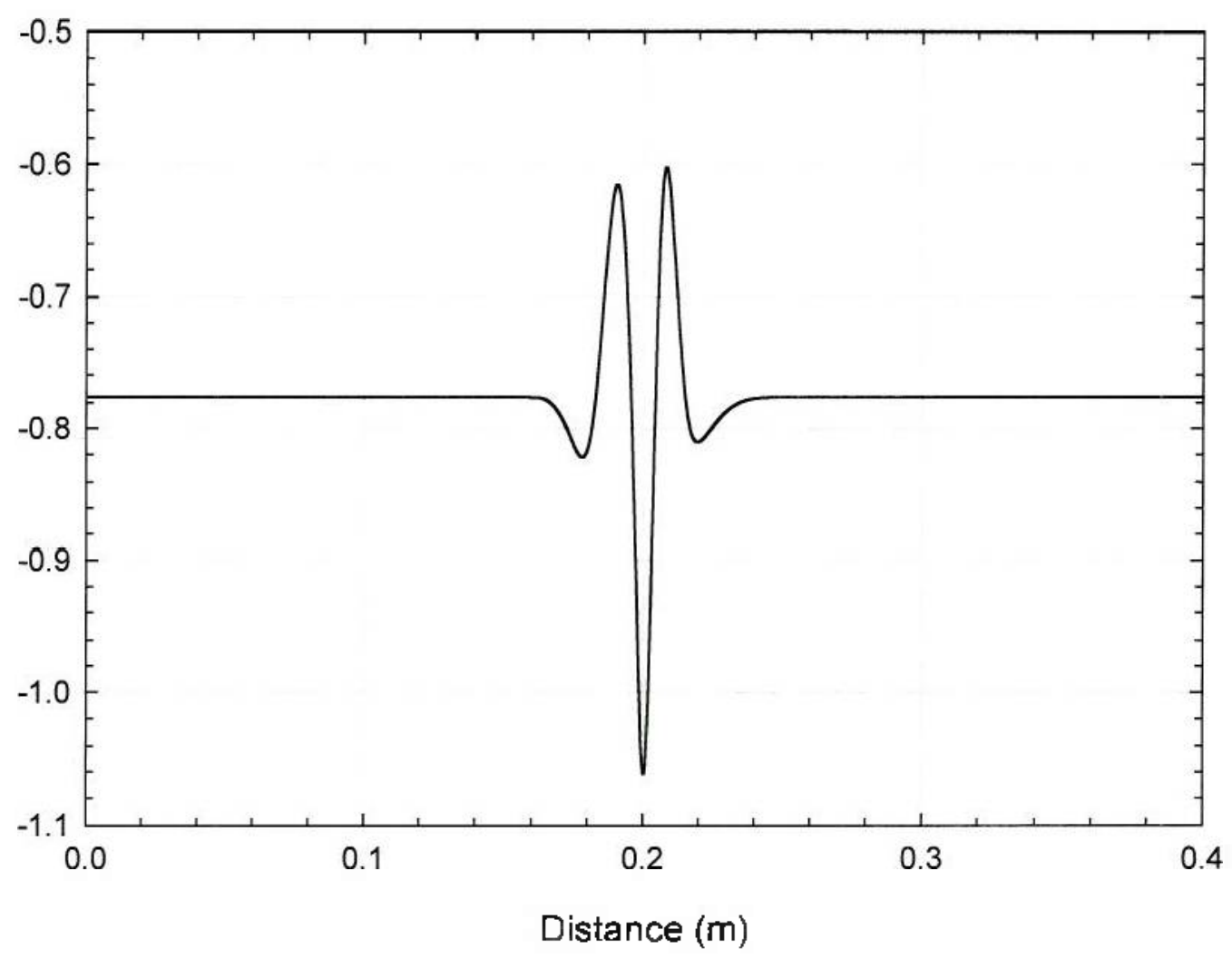

Figure D5: Stress profile in a $40 \mathrm{~cm}$ sample $130 \mathrm{~ms}$ after shock initiation.

\section{Conclusion}

A mathematical model for wave propagation in porous media has been developed and tested. This model uses the mixture theory to formulate mass, momentum, and energy equations in porous media. This model can be used to determine the extent to which remediation is expected to take place. This will aid in the design of sonication tool and in determining the optimum scenario for running this tool. The model was validated using a shock tube problem and the agreement was good. 


\title{
Appendix E. DETAILS ON THE DEVELOPMENT OF HIGH RESOLUTION SOLVER FOR HYPERBOLIC PDES. (Paper submitted for publication on May, 2004). A TVD-Scheme based Generalized Solver for Hyperbolic PDEs
}

\author{
Daniel E. León ${ }^{\mathrm{a}}$ and Michael A. Adewumi ${ }^{\mathrm{b} *}$ \\ ¿Petroleum and Natural Gas Engineering. The Pennsylvania State University, 230 Hosler Building. Universiry Park, PA16802. USA. e- \\ mail:del]92@psu.edu \\ 'Petroleum and Natural Gas Engineering, The Pennsylvania State Universiry, 202 Hosler Building, Universily Park, PAJ6802, USA. e-mail: \\ m2a@psu.edu
}

SUMMARY

\begin{abstract}
Hyperbolic systems of PDEs arise in many practical problems. Thereforc, the description and validation of a generalized onedimensional hyperbolic PDEs solver are presented. This study utilizes the splitting approach to handle the presence of forcing functions, using the Harten's TVD scheme for solving the homogeneous system of PDEs, and the fifth-order Cash $\&$ Karp Embedded Runge-Kutta method for solving the source problem, which is a system of ODEs. The outcome is an efficient, maintanable, and useful program that minimizes the data required, and can be adaptable to any pnoblem without altering the original code. The solver was validated by solving the most general case of a system of hyperbolic PDEs and its simplifications. among which are some classical Benchmark problems like the Linear Advection, Inviscid Burgers equation, Euler equations and a non-homogeneous scalar model equation; moreover, the numerical solutions are compared against their analytica] counterparts. For the specific case of a non-homogeneous system, the Detonation Analogue proposed by Fickett has been used in the past, but we found it to be improper to validate our solver. Hence, due to the lack of problems of this type with exact solution, we have designed our own non-homogeneous system with its analytical solution, which make it useable as a Benchmark problem for this solver and future studies.
\end{abstract}

KEY WORDS: non-homogeneous hyperbolic systems; Total variation diminishing (TVD); Benchmark problems

\section{INTRODUCTION}

Since the beginning of the $20^{\text {th }}$ century, a lot of work has been dedicated to the study of numerical schemes for solving differential equations because most of the complex mathematical expressions of this kind do not have analytical solutions in the literature. In parallel, the developments of the computer along this period has had a great impact on the way principles from the sciences of fluid mechanics and heat transfer are applied to design problems in modern engineering practice. As a result, a new methodology for attacking these complex problems using numerical techniques, known as Computational Fluid Dynamics (CFD), have rise.

Different physical processes governing a varicty of problems can be modeled by hyperbolic partial differential cquations (PDEs). In the Petroleum and Natural Gas field, classical problems involve the flow of single- or multiphase gas in pipclines with either one or more components. In order to solve this kind of problems numerically we need to have the mathematical formulation of the governing equations and the numerical schemes to solve the cquations. Note, that fully-transient problems are modeled by PDEs since they have ac leas two independent variables, namely time and one spatial dimension, while one-dimensional, sceady state formulacions (i.e. no timedcpendence) are modeled by ordinary differential equations (ODFs).

" Correspondence to: Peiroleum and Narural Gas Engineering Program. Department of Energy and Geo-Environmental Engineering, The Pennsy/vania State University, 202 Hosier Building. University Park. PA 16802, USA Tel.; 1814 8653526; Fax: 1814863 I875 
There are various choices for the set of variables to be used in the formulation of the problem. One obvious choice is the set of conserved variábles. Non-consenvarive variables can also be used, but these kinds of formulations fail at shock waves, giving wrong shock strengths, wrong shock speeds and thus wrong shock positions. Moreover, it is also known that non-conservative schemes do not converge to the correct solution if a shock wave is present in the solution [1].

The idea of a generalized solver, using conserved variables allows us to solve different problems without the need to write a new program for every specific case. Therefore, an efficient and useful solver was developed, which minimizes the data required, and can also be adaptable to any problem without altering the original code. This article presents principles governing the generalized solver. The solver is validated with some classical Benchmark problems and; due to lack of non-homogeneous systems with exact solutions in the literature, we designed our own problem with its analytical solution. to be used as a Benchmark problem for the solver.

Another important issue to be handled is the inclusion of an external subroutine, which will permit one to decouple the conserved variables into physical or measurable variables of the problem being solved.

\section{DESCRIPTION OF THE SOLVER}

We suppose that the problems of interest can be modeled using a system of one-dimensional hyperbolic PDEs (1).

$$
\frac{\partial U}{\partial t}+\frac{\partial(F(U))}{\partial x}=Q(U)
$$

Where, $U$ is the vector of conservative variables, $F(U)$ is the vector of physical fluxes, and $Q(U)$ is the vector of forcing functions. The numerical methods available for hyperbolic equations, either first- or high-order accurate are designed to solve only homogeneous systems of the type (2), where the RHS is equal to zero, i.e. there are no source terms or forcing functions.

$$
\frac{\partial U}{\partial t}+\frac{\partial(F(U))}{\partial x}=0
$$

However, equation (1) represents a non-homogeneous system of hyperbolic PDEs. In order to solve it, we need to implement a splitting scheme that solves in different steps the homogencous problem, and the source problem which is another simplification of (1), where the spatial partial derivative is also equal to zero, collapsing to the system of ODEs:

$$
\frac{d U}{d t}=Q(U)
$$

The description of the solver is presented below, and the single-phase Gas Dynamics Euler equations for an ideal gas are used as an example to show the data required by the program and the calculations performed by the routines.

\subsection{Data Reading}


Required Data. There is a minimum amount of data, required by the solver for the solution of the problem. These minimum requirements are presented in Table 1 :

Table I. Minimum requirements for the Solver

\begin{tabular}{|c|c|c|}
\hline Name & Symbol or Description & Example \\
\hline Number of Equations & $m$ & 3 \\
\hline Constifute Variables & $\left(U_{1}, U_{2}, \bar{U}_{3}, \ldots, U_{m}\right)$ & $\begin{array}{c}\left.U=\mid \begin{array}{l}U_{1} \\
U_{2} \\
U_{3}\end{array}\right]=\left[\begin{array}{c}\rho \\
\rho u \\
E\end{array} \mid\right. \\
\text { where: } E=\rho\left(\frac{u^{2}}{2}+e\right), e=\frac{p}{(\gamma-1) \rho}, \quad \gamma=c_{n} / c_{\nu}\end{array}$ \\
\hline Physical Fluxes & $\begin{array}{l}F_{1}=F_{1}\left(U_{1}, U_{2}, \ldots, U_{m}\right) \\
F_{2}=F_{2}\left(U_{1}, U_{2}, \ldots, U_{m}\right) \\
\vdots \\
F_{m}=F_{m}\left(U_{1}, U_{2}, \ldots, U_{m t}\right)\end{array}$ & $\begin{array}{c}\left.F(U)=\left[\begin{array}{c}F_{1} \\
F_{2} \\
F_{3}\end{array}\right]=\left|\begin{array}{c}\rho u \\
\rho u^{2}+p \\
u(E+p)\end{array}\right|=\mid \begin{array}{c}U_{2} \\
\frac{U_{2}^{2}}{U}+(\gamma-1)\left(U_{3}-\frac{U_{2}^{2}}{2 U_{1}}\right) \\
\frac{U_{2}}{U_{1}}\left(U_{3}+(\gamma-1)\left(U_{3}-\frac{U_{2}^{2}}{2 U_{1}}\right)\right)\end{array}\right] \\
\text { where: } p=(1-\gamma)\left|U_{3}-\frac{U_{2}^{2}}{2 U_{1}}\right|\end{array}$ \\
\hline Consrants & & $\gamma$ \\
\hline Simulation Time & \multicolumn{2}{|l|}{ TotalT } \\
\hline CFL Number & \multicolumn{2}{|l|}{$0<\mathrm{CHL}<1$} \\
\hline $\begin{array}{l}\text { Accuracy-order of the } \\
\text { splitting approach: }\end{array}$ & \multicolumn{2}{|c|}{ First- or second-order accurate } \\
\hline $\begin{array}{l}\text { Length of the spatial } \\
\text { domain }\end{array}$ & \multicolumn{2}{|l|}{$L$} \\
\hline Number of gridpoints & \multicolumn{2}{|l|}{$N$} \\
\hline $\begin{array}{l}\text { Boundary conditions } \\
\text { and type }\end{array}$ & \multicolumn{2}{|c|}{ Transmissive Neumann-type $\left(\left.\frac{\partial t i}{d x}\right|_{L \ldots \ldots}-0\right)$ or Drichlet-type $\left(\left.U\right|_{\ldots \ldots}=C\right)$} \\
\hline Initial conditions: & \multicolumn{2}{|c|}{$\begin{array}{l}\text { There are two options for the initial conditions. One is the classical Riemann problem. } \\
\text { and the other possibility is to oblain the steady-state distribution: } \frac{d F(U)}{d x}=Q(U)\end{array}$} \\
\hline
\end{tabular}

External subroutine: Due to the generalized property of the code, the solutions generated by the solver are always the values of the conserved variables $(U)$. Therefore, in order to obtain the values of physical or measurable variables for the specific problem such as pressure, temperature, velocity, internal energy, holdup, phase composition, etc; the user has to provide a FORTRAN subroutine that receives the vector $U$ and decouples it into desired variables. Morcover, for more general problems, the decoupling process will occur at the end of each time step, when the source terms need to he evaluated 
Optional Data. There are some additional data that might be available for certain problems, and when they are provided, the computational time needed to generate them is saved, allowing it to solve the problem These data are described in Table 2.

Table 2. Optional data for the Solver.

\begin{tabular}{|c|c|c|}
\hline Name & Symbol or Description & Example \\
\hline $\begin{array}{l}\text { Jacobian } \\
\text { Matrix }\end{array}$ & 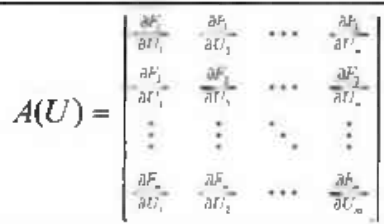 & 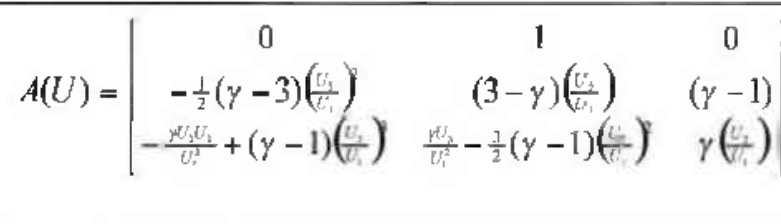 \\
\hline Eigenvalues & $\begin{array}{l}\lambda_{1}=\lambda_{1}\left(U_{1}, U_{2}, \ldots, U_{m}\right) \\
\lambda_{1}=\lambda_{2}\left(U_{1}, U_{2}, \ldots, U_{m}\right) \\
\vdots \\
\lambda_{m}=\lambda_{m}\left(U_{1}, U_{2}, \ldots, U_{m}\right)\end{array}$ & $\begin{array}{l}\lambda_{4}=u-a ; \quad \lambda_{1}=u ; \quad \lambda_{1}=u+a \\
\text { where: } a=\sqrt{\frac{p p}{\rho}}\end{array}$ \\
\hline $\begin{array}{l}\text { Righr } \\
\text { Eigenvectors }\end{array}$ & $\begin{array}{l}R^{(1)}=\left|\begin{array}{l}R_{1}^{(1)}\left(U_{1}, \ldots, U_{m}\right) \\
R_{2}^{(1)}\left(U_{1}, \ldots, U_{m}\right) \\
\vdots \\
R_{m}^{(1)}\left(U_{1}, \ldots, U_{m}\right)\end{array}\right| ; \\
R^{(2)}=\left|\begin{array}{l}R_{1}^{(2)}\left(U_{1}, \ldots, U_{m}\right) \\
R_{2}^{(2)}\left(U_{1}, \ldots, U_{m}\right) \\
\vdots \\
R_{m}^{(2)}\left(U_{1}, \ldots, U_{m}\right)\end{array}\right| ; \\
R^{(m)}=\left|\begin{array}{l}R_{1}^{(m)}\left(U_{1}, \ldots, U_{m}\right) \\
R_{2}^{(m)}\left(U_{1}, \ldots, U_{m}\right) \\
\vdots \\
R_{m}^{(m)}\left(U_{1}, \ldots, U_{m}\right)\end{array}\right|\end{array}$ & $\begin{array}{l}R^{(1)}=\left|\begin{array}{c}1 \\
u-a \\
H-u a\end{array}\right| ; \quad R^{(2)}=\left|\begin{array}{c}1 \\
u \\
\frac{y^{2}}{7}\end{array}\right| ; \quad R^{(1)}=\left|\begin{array}{c}1 \\
u+a \\
H+u a\end{array}\right| \\
\text { where: } H=\frac{E+p}{\rho}\end{array}$ \\
\hline
\end{tabular}

\subsection{Grid Type and Boundary Conditions}

The grid type is chosen by the program depending upon the type of boundary condition imposed. If the boundaries are transmissive Neumann-type. a block-centered grid (Figure 1) is assigned to the domain, and the corresponding houndary conditions are applied according to (4).

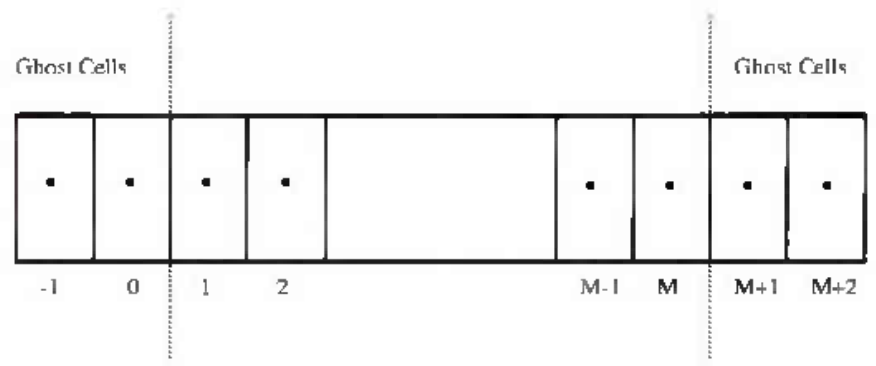

Figure I. Schemalic of a block-centered grid. 


$$
\text { Inner BCs : }\left\{\begin{array}{l}
U_{-1}=U_{2} \\
U_{0}=U_{1}
\end{array} ; \text { Outer BCs }:\left\{\begin{array}{l}
U_{M+2}=U_{M-1} \\
U_{M+1}=U_{M}
\end{array}\right.\right.
$$

On the other hand, if Drichlet-type boundaries exist. a point-distributed grid (Figure 2) is used.

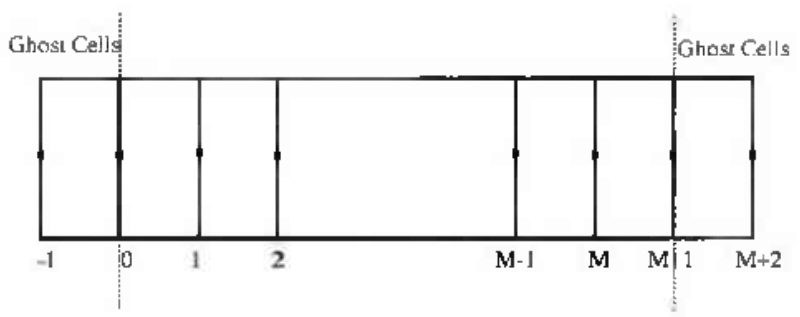

Figure 2. Schematic of a point-distributed grid.

\subsection{Homogeneous Hyperbolic PDE Solver}

As mentioned before, the numerical merhods to solve hyperbolic PDEs, are designed to handle homogeneous systems (2) only. Therefore, when solving general problems of the form (1), one has to solve in one step the homogeneous problem, which is presented in this section, and the source problem (3) in a different step as we will sec later.

Eigenstructure of the System. For classical problems, the eigenstructure of the system is well known, and it can he provided to the solver as an optional data. But, for most general problems that this program is meant to solve, no analytical expressions can be devised to obrain the eigenvalues and corresponding right eigenvectors. In such cases, the solver can generate them numerically as shown below.

a) Jacobian Matrix: Having the flux functions written in terms of the conserved variables, the partial derivatives of the Jacobian are oblained numerically using a first-order approximation of partial derivate, namely:

$$
\frac{\partial F_{1}}{\partial U_{j}}=\frac{F_{j}\left(U_{1}, U_{2}, \ldots, U_{1}+\varepsilon_{j}, \ldots, U_{m}\right)-F_{1}\left(U_{1}, U_{2}, \ldots, U_{j}, \ldots, U_{m}\right)}{\varepsilon_{j}}
$$

As an example, for the Fuler equation we have:

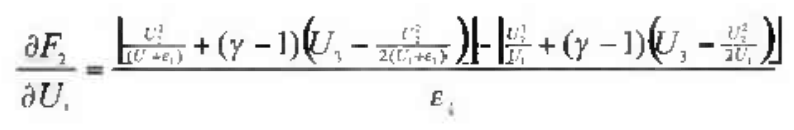

As a default, all the epsilons $\left(\varepsilon\right.$, ) in the program have been set equal to $1.10^{-7}$, but they can be changed according to the magnitude of the variables of the problem being solved. 
b) Eigenvalues: The program making use of the definition of strictly hyperbolic system, which says that the matrix $A(U)$ has $m$ real and distinct eigenvalues. obtains the eigenvalues numerically. Having the Jacobian matrix, the characteristic polynomial of $\mathrm{m}^{\text {th }}$-order is defined as:

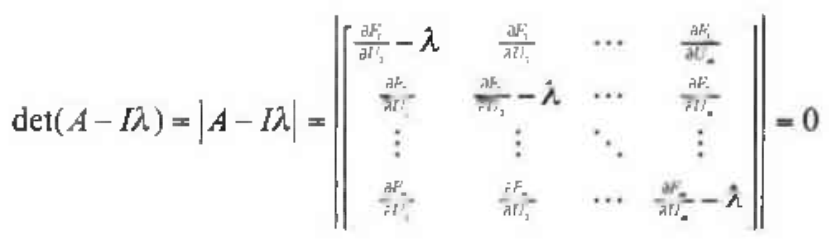

Then, the determinant of a square matrix can be expressed in terms of its minors, like:

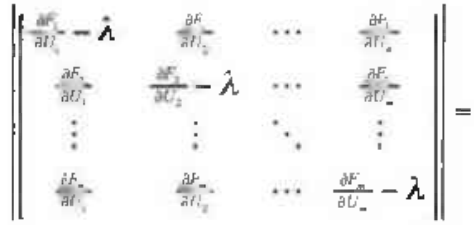

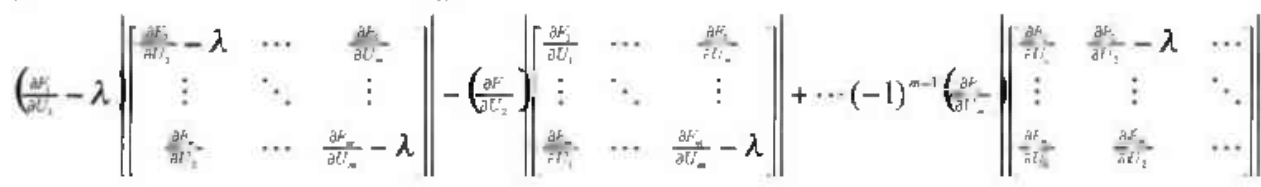

Obviously, during the first time step none of the eigenvalues are known at any of the intercells, and thus the searching is more time consuming, particularly the first one. For that first intercell the search starts at a point in the real domain theoretically tocated in $-\infty$, (i.e. $-10,000$ ). Then, at a fixed step (i.e. 0.01 ) the search is performed by rying to satisfy the definition (7). During the search, once the value given by the determinant change its sign from one step to another, it implies that the interval where the first root is located, has been found. Finally, the eigenvalue is determined to the accuracy of $1.10^{-12}$ using Bisection method. After that, the search continues from that point and the remaining eigenvalues are found using the same fixed step.

It is clear that the search for the eigenvalues is critical and time consuming during the first time step, but for the subsequent time steps, the eigenvalues corresponding to the previous one are use as initial guesses, making the search process more efficient. Furthermore, since the time step in this kind of systerns is very small, $\Delta t=10^{-3} \sim 10^{-3}$, the guess values are very close to the actual ones.

c) Right Eigenvectors: Any matrix has an associated polynomial whose roots are the eigenvalues of the matrix. The reverse is also true: for any polynomial, there is an associated matrix whose eigenvalues are the same as the roots of the polynomial. However, "the correspondence between polynomial and matrices cannot be one-to-one" [3]. This is because a general matrix of order $m$, depends on $m^{2}$ parameters (its entries), whereas a polynomial of degree $m$ depends on only $m+1$ parameters (its cocfficients). Thus, distinct matrices can have the same characteristic polynomial. The right eigenvectors of a system are defined as column vectors, $R^{(i)}$, satisfying the relation:

$$
A \cdot R^{(i)}=\lambda_{1} \cdot R^{(i)}
$$


An important feature is that the eigenvectors are not unique, so they can be scaled arbitrarily. This means that if a vector $R^{(1)}$ satisfy $(9)$, then for any scalar $\gamma \neq 0, \gamma \cdot R^{(1)}$ is also a right eigenvector corresponding to $\lambda_{1}$. Consequently, eigenvectors are usually normalized by requiring some norm of the vector to be 1 .

What the above statement implies is that two out of the $m$ equations defined by $(10)$ are equivalent. Therefore, one of these two equations is eliminated, then the first component of the eigenvector is set to 1 , and the resulting ( $m-[\times m-1$ ) linear system (15) is solved exactly using the Gauss-Jordan method. Mathernatically, expanding (9) gives:

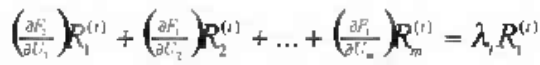

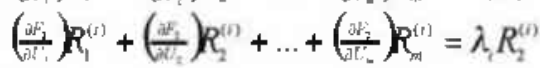

$$
\begin{aligned}
& \text { : }
\end{aligned}
$$

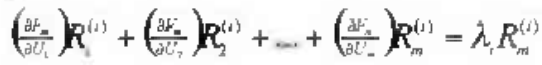

After eliminating one of the equations and rearranging the system in the form $[A]_{\text {wem-1 }}[x]_{-1}=[b]_{-1}$, one has:

$$
\begin{aligned}
& R_{1}^{(i)}=1
\end{aligned}
$$

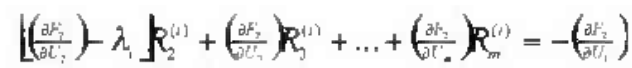

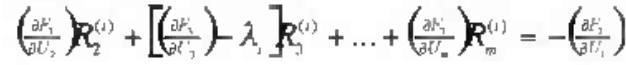

$$
\begin{aligned}
& \text { : }
\end{aligned}
$$

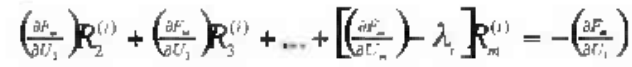

Modified Numerical Flux. Harten [4] circumvented Godunov's theorem, which concluded that "monotone schemes are at most first-order accurate" $|\mathrm{l}|$. Having a first-order upwind scheme, with a truncation error of the form:

$$
\begin{aligned}
& \frac{\partial u}{\partial t}+\frac{\partial f(u)}{\partial x}=\Delta t \frac{\partial}{\partial x}\left|\beta(u, \sigma) \frac{\partial u}{\partial x}\right| ; \quad \sigma=\frac{\Delta t}{\Delta x}, \\
& \beta(u, \sigma)=\frac{1}{2 \sigma^{2}}\left[\sum_{i=1}^{k} j^{2} H_{j}\left(u, u_{1}, \ldots, u\right)-\sigma^{2} a^{2}(u) \mid\right.
\end{aligned}
$$

Its solutions are second-order accurate approximations of this equivalent differential equation. Hence, "if the truncation error is subtracted from the original flux as an antidiffusive flux, the solucions of the resulting equations will be second-order approximations of the original equation" [5], i.e.

$$
\frac{\partial u}{\partial t}+\left(\frac{\partial f}{\partial x}-\frac{1}{\sigma} \frac{\partial g}{\partial x}\right)=0, \quad g(x)=\Delta x \beta(u, \sigma) \frac{\partial u}{\partial x}
$$

Now, the application of the original first-order scheme to the conservation law with the modified flux function $(f+g)$ gives: 


$$
\frac{\partial u}{\partial t}+\left(\frac{\partial f}{\partial x}+\frac{1}{\sigma} \frac{\partial g}{\partial x}\right)=0
$$

Which is a second-order approximation of the scalar case of (2). Then. Harten [4] defined a second-order accurate modified numerical flux and expanded it to systems of conservation laws as:

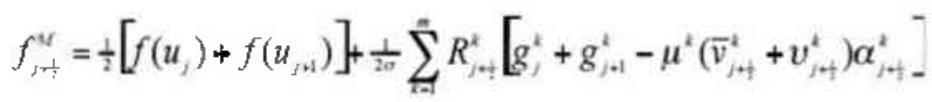

Note that the right eigenvectors in the above equation correspond to the intercell, and although Roe [6] stated that the choice of an average matrix of the form $\widetilde{A}(U)=A\left(\frac{1}{3}\left(U_{f}+U_{R}\right)\right)$ would not be appropriate, all our experiments agree with Harten [4] in the sense that the arithmetic average works as well as the Roe's linearization. The wave strengths, $\alpha_{j+\dagger}^{k}$ can be obtained analytically by solving the local Riemann problem through decomposing the data jump onto the right eigenvectors as:

$$
\Delta U_{i}=U_{i R}-U_{i L}=\sum_{\gamma=1}^{m} \alpha^{(t)} R_{i}^{(j)}
$$

Expanding (16), for every intercell, we have a system of $m$ equations (17) which is solved using the Gauss-Jordan method.

$$
\begin{aligned}
& \Delta U_{1}=U_{1 R}-U_{1 L}=\alpha^{(1)} R_{1}^{(1)}+\alpha^{(2)} R_{1}^{(2)}+\cdots+\alpha^{(m)} R_{1}^{(m)} \\
& \Delta U_{2}=U_{2 R}-U_{2 I}=\alpha^{(1)} R_{2}^{(1)}+\alpha^{(2)} R_{2}^{(2)}+\cdots+\alpha^{(m)} R_{2}^{(m)} \\
& \vdots \\
& \Delta U_{m}=U_{m R}-U_{m L}=\alpha^{(1)} R_{m}^{(1)}+\alpha^{(2)} R_{m}^{(2)}+\cdots+\alpha^{(m)} R_{m}^{(m)}
\end{aligned}
$$

Then, the following functions are computed in the given order:

$$
\begin{aligned}
& \bar{v}_{j+\downarrow}=\frac{\Delta}{\Delta x} \lambda_{\lambda}\left(u_{k j+1}\right) \\
& \tilde{g}_{j+\frac{1}{2}}^{k}=\frac{1}{2}\left[\mu^{k}\left(v_{j+\frac{1}{2}}^{k}\right)-\left(v_{j-\frac{1}{2}}^{k}\right)^{2}\right] \alpha_{j+\frac{1}{2}}^{k} \\
& s_{j+\frac{1}{2}}^{k}=\operatorname{sigh}\left(\tilde{g}_{j+\frac{1}{2}}^{k}\right) \\
& g_{j}^{k}=s_{j+\frac{2}{3}}^{k} \max \left[0, \min \left(\left|\vec{g}_{s+\frac{1}{2}}^{k}\right|, \tilde{g}_{j-j_{j+}^{k}}^{k} s^{k}\right)\right]
\end{aligned}
$$

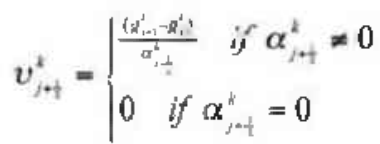

Note that $\lambda_{i}$ and $R^{k}$ are the eigenvalues and corresponding right eigenvectors of the system respectively. And, $\mu(v)$ is a funcrion inside the viscosity coefficient, whose natural choice is $\mu(v)=|\nu|$ to give the less dissipative TVD scheme of the form (15). Howcver, when the numerical viscosity vanishes for $v=0$ an entropy violation appears in the scheme. Therefore, it seems possible to eliminate this sort of entropy violation by simply modifying 
the function to be positive near $v=0$. To make $u(v)$ smoother at the same time Harten [4] defined it in the interva] $0<\delta<\frac{1}{3}$, ás:

$$
\mu(v)= \begin{cases}\frac{\nu^{2}}{d \delta}+\delta & \text { if }|v|<2 \delta \\ |v| & \text { if }|v| \geq 2 \delta\end{cases}
$$

In his tests, Harten [4] used values of $\delta=0.05,0.1$ and 0.25 for the case of single-phase Euler equations and, although the dependence on $\delta$ is slight, he selected $\delta=0.1$ for genuinely non-linear fields. Finally, what we have is a modified numerical flux, to be used for solving any system of non-linear hyperbolic equations using the conservative formula (25).

CFL Number and Timestep size. According to Tannehill et al [7], the beginning of modern numerical analysis could be attributed to the famous paper by Courant, Friedrichs and Lewy [2], in whose names stand the acronym CFL.. The application of the von Neumann or Fourier stability method to the first-order wave equation (20). produces the numerical stability requirement (22) for explicit methods of the form (25).

$$
\begin{gathered}
\frac{\partial u}{\partial t}+a \frac{\partial u}{\partial x}=0 \\
\left|a \frac{\Delta t}{\Delta x}\right| \leq 1
\end{gathered}
$$

The spatial discretization length ( $\Delta x$ ) is chosen for a combination of desired accuracy and availahle computing resources. From (22), the timestep size can he ohtained as:

$$
\Delta t=\frac{C_{c} \Delta x}{S_{\max }^{n}}
$$

Where, $C_{c f}$ is the CFI coefficient satisfying the restriction $0<C_{\omega n} \leq 1$. Note that, as the coefficient $C_{C A}$ is closer to I, the scheme becomes more efficient. Furthermore, $S_{\text {max }}^{n}$ is the largest wave speed present along the domain at time level $n$, which in our solver is nothing more than the largest eigenvalue, namely:

$$
S_{-\infty}^{n}=\max \left\{\left|\lambda^{n}\right|\right\}
$$

Updating the Solution. For the conservation laws (2), a control volume $V=\left[x_{i}, x_{R}\right] \times\left[t_{1}, t_{2}\right]$ is defined on the $\mathrm{x}-\mathrm{t}$ plane (Figure 3), and the equations can be written in the integral form (24). 


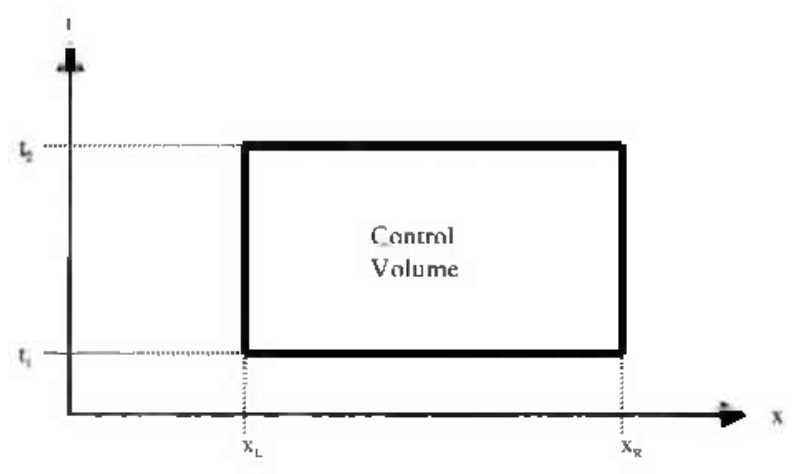

Figure 3. Control volume $V=\left[x_{1}, x_{3}\right] \times\left[t_{1}, t_{2}\right]$ on the $x-t$ plane $\left.\mid 1\right]$.

$$
\int_{x_{L}}^{\pi_{5}} U\left(x, t_{2}\right) d x=\int_{x_{1}}^{\pi_{\pi}} U\left(x, t_{1}\right) d x+\int_{4_{4}}^{t_{4}} F\left(U\left(x_{L}, t\right)\right) d t-\int_{5}^{4} F\left(U\left(x_{R}, t\right)\right) d t
$$

Finally, after integrating along the discrete volume, a conservative scheme is defined as a numerical method of the form:

$$
U_{1}^{n+1}=U_{1}^{n}+\frac{\Delta \Delta}{\Delta x}\left(F_{i-Y_{1}}^{n}-F_{i+y_{1}}^{n}\right)
$$

The conservative formula (25) is used to calculate the solution at the new timestep " $n+1$ " using the Harten's modified flux (15).

\subsection{Splitting Scheme for Non-homogenous Systems}

Non-homogeneous systems of the form (1), arise in many practical problems that can be solved by this program. The approach followed by this work is the splitting scheme, which solves in two different steps the homogeneous problem (2), and the source problem (3).

Justification of the method. For the simplest non-homogeneous hyperbolic equation given by the IVP (26), where $a$ is the constant speed of the wave and $\eta$ is another constant parameter, the splitting apptoach gives an exact solution, and based on computational experience it has been demonstrated that this technique also gives appropriate solutions for more general problems [1].

$$
\left.\begin{array}{l}
P D E: \quad \frac{\partial u}{\partial t}+a \frac{\partial u}{\partial x}=\eta \eta \\
\text { /C: } u(x, 0)=u_{0}(x)
\end{array}\right\}
$$

The exact solution of (26) is:

$$
\left.u(x, t)=u_{\mathrm{n}}(x-\sigma)\right) e^{\eta t}
$$


It is also important to note that when $\eta=0$ the exact solution collapses to the one for the homogencous equation, namely the linear advection problem. By splitting the problem into a pair of IVPS (28) and (29):

$$
\begin{aligned}
& \left.\begin{array}{l}
P D E: \quad \frac{\partial r}{\partial \ell}+a \frac{\partial r}{\partial x}=0 \\
I C: \quad r(x, 0)=u_{0}(x)
\end{array}\right\} \Rightarrow r(x, t) \\
& O D E: \quad \frac{d}{d t} s=\eta, \\
& I C: \quad s(0)=r(x, t)
\end{aligned} \mid \Rightarrow s(t)
$$

The solution of the homogeneous problem (28) is $r(x, t)=u_{0}(x-a t)$, while the solution of the source problem (29) is given by $s(x(t), t)=s(0) e^{\pi}$. Since $s(0)=r(x, t)=u_{0}(x-a t)$ the resulting solution of the original problem (26) is:

$$
s(x(t), t)=u_{0}(x-a t) e^{n t}
$$

Note that this is the exact solution (27) given before. What we have applied is the splitting scheme, which solves in succession (28) and (29), and can be expressed as:

$$
u(x, t)=O^{(t)} P^{(t)}\left[u_{0}(x)\right]
$$

Where $P^{(1)}$ is the homogeneous hyperbolic PDE operator applied over a lime $t$, and $O^{(t)}$ is ODE operator also applied on the timeframe $t$.

Numerical Methods based on Spliting. An exact solution, like the one generated for the model equation (26), can no longer be obtained when applying the scheme to non-linear systems like (1). However, good approximate numerical solutions can be obtained when using the method for discrete data. The solution is computed by evolving the initial value $U^{n}$, by one time step of size $\Delta t$, to a value $U^{n=1}$ in a spatial domain $[0, L]$, which has been discretized into a finite number $N$ of gridpoints. Having a systern (32), the steps (28) and (29) can be generalized as (33) and (34) respectively.

$$
\begin{aligned}
& P D E s: \quad \frac{\partial U}{\partial t}+\frac{\partial F(U)}{\partial x}=Q(U) \quad, 0 \leq x \leq L \\
& \text { ICs : } \quad U\left(x, t^{n}\right)=U^{n} \\
& \left.\begin{array}{l}
\text { PDEs : } \quad \frac{\partial U}{\partial t}+\frac{\partial F(U)}{\partial x}=0 \\
\text { ICS }: \quad U\left(x, t^{n}\right)=U^{n}
\end{array}\right\} \Rightarrow \bar{U}^{n+1} \\
& \left.\begin{array}{l}
\text { ODES: } \quad \frac{d}{d t} U=Q(U) \\
\text { ICS : } \bar{U}^{n+1}
\end{array}\right\} \Rightarrow U^{n+1}
\end{aligned}
$$

For numerical methods, analogous to (31), a first-order accurate version of the splitting approach is: 


$$
U^{n-1}=O^{(\Delta)} P^{(\Delta)}\left[U^{n}\right]
$$

Moreover, if both the $P$ and $O$ operators are at least second-order accurate, (36) is a second-order accurate splitting scheme.

$$
U^{n+1}=O^{\left(\frac{\alpha}{2}\right)} P^{(\Delta)} O^{\left(\frac{*}{2}\right)}\left[U^{n}\right]
$$

At this point it is important to point out that the beauty of the splitting scheme is in the freedom to choose the numerical operators $P$ and $O$. In general, one may choose the best scheme for each type of problems. This study adopted the Harten's TVD scheme for solving the homogeneous IVPs (2), and the fifth-order Cash \& Karp Embedded Runge-Kutta method for solving the ODEs (3).

\subsection{ODE Solver}

The explicit Runge-Kutta method is one of the most well known for solving ODEs. A fifth-order accurate estimate of the solution is obtained by the Runge-Kutta-Fehlberg method:

$$
\left.U(s+\Delta s)\right|_{s^{2}}=U_{0}+\sum_{i=1}^{b} a_{r}^{\gamma} F_{i}^{*}
$$

Where $a_{q}^{k}$ are constant coefficients proposed by Fehlberg [8], and the quantities $F_{i}^{f}$ are compuled by evaluating the RHS vector with formulas of the type:

$$
F_{i}^{+}=\Delta s \cdot R H S\left(U_{0}+\sum_{j=1}^{H} d_{v}^{F} F_{j}^{F}\right), 1 \leq i \leq 6
$$

The constant coefficients $d_{y}^{*}$ are also given by Fehlherg [8]. Note that to gel al] the $F_{i}^{f}$ quantities, six evaluations of the RHS vector are required. Once all these quantities are known, the final solution is calculated from (37).

The original values of the constants ( $a_{t}^{F}, d_{y}^{F}$ ) are given by Fehlberg |8|, but according to Press et al |9], Cash and Karp [10] proposed ones that produce a more efficient method, with better error properties (Table 3) and those are the ones chosen for our program.

Table 3. Cash-Karp | 10 | parameters for embedded Runge-Kutta method

\begin{tabular}{|c|c|c|c|c|c|c|}
\hline$i$ & $a_{1}$ & \multicolumn{3}{|c|}{$d_{i j}$} & \\
\hline 1 & $\frac{32}{378}$ & & & & & \\
\hline 2 & 0 & $\frac{1}{5}$ & & & & \\
\hline 3 & $\frac{250}{621}$ & $\frac{3}{40}$ & $\frac{9}{40}$ & & & \\
\hline 4 & $\frac{125}{594}$ & $\frac{3}{10}$ & $-\frac{9}{10}$ & $\frac{6}{5}$ & & \\
\hline 5 & 0 & $-\frac{11}{54}$ & $\frac{5}{2}$ & $-\frac{70}{27}$ & $\frac{35}{27}$ & \\
\hline
\end{tabular}




\begin{tabular}{|c|c|c|c|c|c|c|}
\hline 6 & $\frac{512}{1771}$ & $\frac{1631}{55296}$ & $\frac{175}{512}$ & $\frac{575}{13824}$ & $\frac{44275}{110592}$ & $\frac{253}{4096}$ \\
\hline & & 1 & 2 & 3 & 4 & 5 \\
\hline
\end{tabular}

\section{BENCHMARK PROBLEMS}

A number of Benchmark problems whose analytical solutions are available is used to validate the solver. Due to the generalized property of the code, problems with essential characteristics of shocks were solved for all the possible simplifications of a system of hyperbolic PDEs. These include homogenous and non-homogeneous, linear and nonlinear systems. Note also that all the four previous problems can be collapses to their corresponding scalar cases. We now present a comparison between the analytical and numerical solutions for classical benchmark problems.

For the case of non-homogeneous systems, the Detonation Analogue problem proposed by Fickett $|11|$ has been utilized in the work done by Clarke et al [12] to choose the numerical scheme. We have found that the reacrion equation in the Detonation Analogue model is an ODE, which makes this problem unsuitable to validate our solver. For that reason we have creared our own system of equations, for which we know the exact solutions, and hence it can bc uscd as a benchmark problem for this program and for future studies.

\subsection{Homogeneous Linear Scalor Case - Linear Advection Equation}

Initially we solved the classical Linear Advection problem, which describes a wave propagaring in the $x$ direction with a velocity $a|7|$. Here the initial data of the IVP (34b)-(53) is a square wave in the spatial domain $[0,2.5] ; 250$ cells were used, taking $a=1.0$, and a CFL number 0.99 .

$$
\text { ICs: } u(x, 0)=u_{0}(x)= \begin{cases}0 & \text { if } x<0.3 \\ 1 & \text { if } 0.3 \leq x \leq 0.7 \\ 0 & \text { if } x>0.7\end{cases}
$$

The exact solution of this problem is such that given an initial distribution of the data $u_{o}(x)$, the PDE translate it with a speed $a$. This is:

$$
u(x, t)=u_{n}(x-a)
$$

The solution of the problem was calculated numerically for two different output times (0.5 and 1.5 ), and the results are shown in Figure 4, where the numerical and exact solutions are compared. 

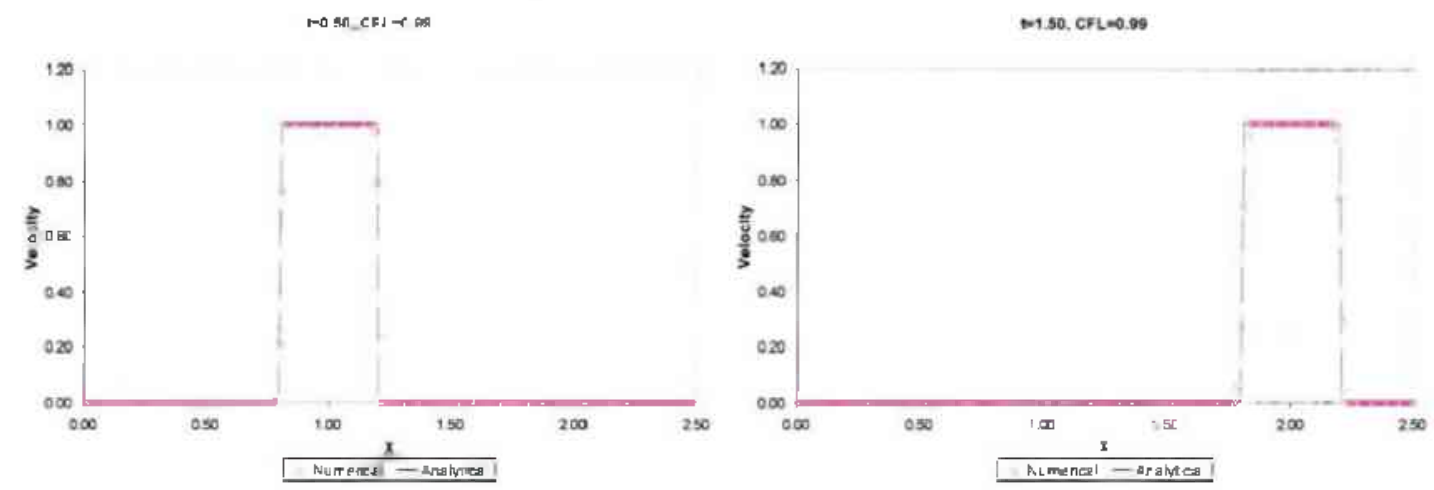

Figure 4. Comparison for the Linear Advection problem.

\subsection{Homogeneous Non-linear Scalar Case - Inviscid Burger's Equation}

Unfortunately, the usual fluid mechanics problem is highly non-linear. As an analog of the non-linear equations that our solver will be dealing with, here we present the solution of the inviscid Burger's equation (41) in the spatial domain $[0,4]$, using 400 cells, and for a CFL number 0.99 .

$$
\begin{gathered}
\text { PDE: } \frac{\partial u}{\partial t}+\frac{\partial f(u)}{\partial x}=0, \quad f(u)=\frac{u^{2}}{2} \\
\text { ICs: } u(x, 0)=u_{0}(x)= \begin{cases}\frac{1}{2} & \text { if } x \leq \frac{1}{4} \\
2 & \text { if } \frac{1}{4}<x<1 \\
1 & \text { if } x \geq 1\end{cases}
\end{gathered}
$$

The numerical solution of the problem was obtained for two output times (0.5 and 1.5), and they are shown in Figure 5.
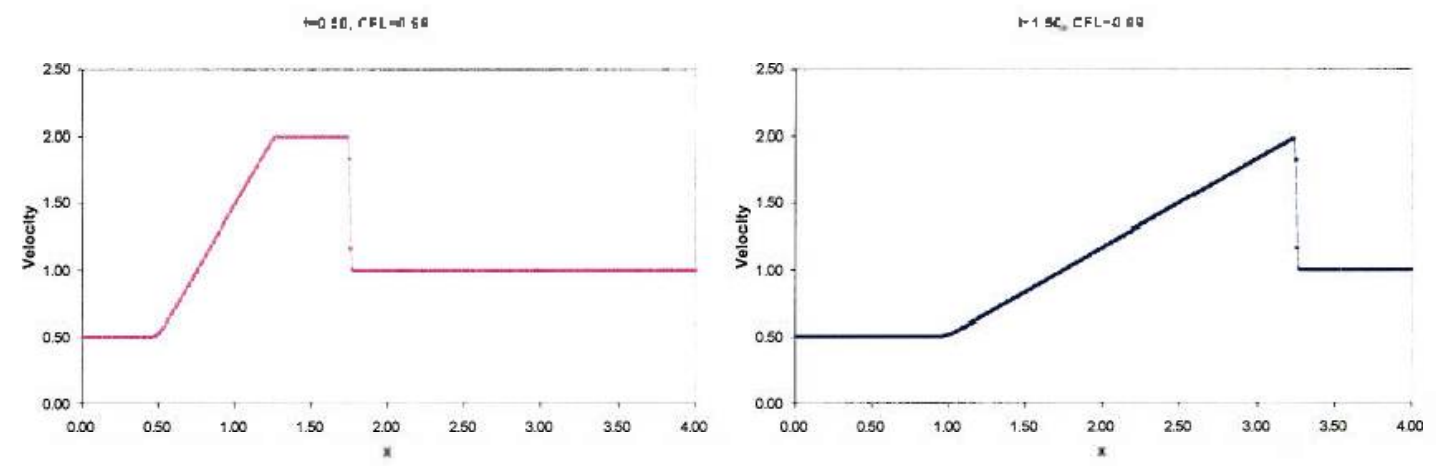

Figure 5. Numerical solution for the Burger's equation.

\subsection{Homogeneous Linear Sysrem - Linearized Gas Dynamics Equations}

Entering in the field of systems of equations, the linearized equations of Gas Dynamics (42), can be derived from the conservations laws that govern the dynarnics of compressible fluids, namely the time-dependent Euler equations [1]. The unknowns of the system are: the density $u_{i}=\langle x, t)$ and the speed $u_{2}=u(x, t)$ of the fluid. The positive 
constants , and $a$ represent the reference density, and the speed of sound respectively. The problem was solved in the spatial domain $[0,2]$, using 200 cells, and for a CFL number 0.67 . The constant values used are $a=1.0, \quad 0=1.0$.

$$
\text { PDEs: } \begin{aligned}
& \frac{\partial \rho}{\partial t}+\rho_{0} \frac{\partial u}{\partial x}=0 \\
& \frac{\partial u}{\partial t}+\frac{a^{2}}{\rho_{0}} \frac{\partial \rho}{\partial x}=0
\end{aligned} \mid
$$

By expanding the LHS and RHS data states in terms of the corresponding eigenvectors, and after some algebraic manipulations the exact solution of the problem can be written as:

$$
\begin{gathered}
\rho(x, t)=\frac{1}{2 a}\left[a \rho^{(0)}(x+a t)-\rho_{o} u^{(0)}(x+a t)\right]+\frac{1}{2 a}\left[a \rho^{(0)}(x-a t)+\rho_{c} u^{(0)}(x-a t)\right] \\
u(x, t)=-\frac{1}{2 \rho_{0}}\left[a \rho^{(0)}(x+a t)-\rho_{0} u^{(0)}(x+a t)\right]+\frac{1}{2 a}\left[a \rho^{(0)}(x-a t)+\rho_{0} u^{(0)}(x-a t)\right]
\end{gathered}
$$

Three different tests were performed, whose initial conditions are included in Table 4.

Table 4. Initial condilions for different tests. Linearized Gas Dynamics equations.

\begin{tabular}{|c|c|c|}
\hline Test & Density & Velocity \\
\hline 1 & $\rho(x, 0)=\rho^{(0)}(x)=\left\{\begin{array}{lll}1.0 & \text { if } & 0<x \leq 1 \\
0.5 & \text { if } & 1<x \leq 2\end{array}\right.$ & $u(x, 0)=u^{(0)}(x)=0$ \\
\hline 2 & $\rho(x, 0)=\rho^{(0)}(x)=\left\{\begin{array}{lll}0.5 & \text { if } & 0<x \leq 1 \\
1.0 & \text { if } & 1<x \leq 2\end{array}\right.$ & $u(x, 0)=u^{(0)}(x)=0$ \\
\hline 3 & $\rho(x, 0)=\rho^{(0)}(x)=0.75$ & $u(x, 0)=u^{(n)}(x)=\left\{\begin{array}{lll}-0.5 & \text { if } & 0<x \leq 1 \\
1+0.5 & \text { if } & 1<x \leq 2 \\
\hline\end{array}\right.$
\end{tabular}

The first test is a problem solved analylically by [1] where two symmetric waves emerge from the initial position of discontinuity, carrying a discontinuous jump in both density and velocity. Test 2, is a slight symmetric modification of test 1 , which was included in order to test the ability of the solver to handle conservative variables with negative signs. The assessment of the solver with conservative variables of mixed signs is presented in test 3.

The solutions of the three tests were calculated for an output time of 0.7 , and the results are shown in Figures 6 to 8 , where the numerical and exact solutions are compared. 

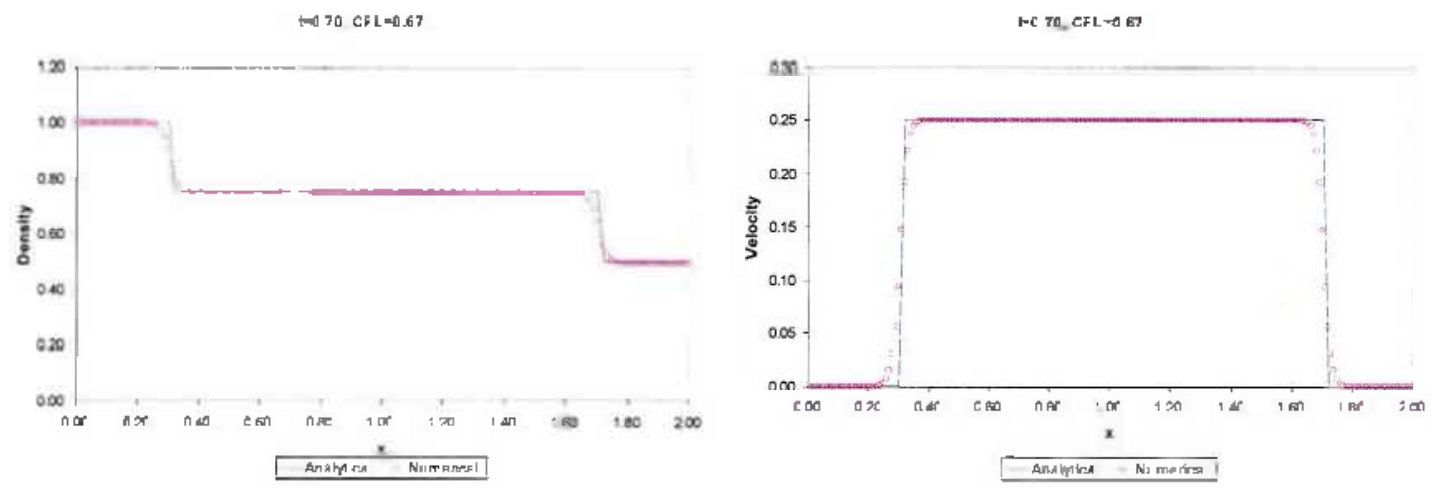

Figure 6. Comparison for the Linearized Gas Dynamics equations (test 1).
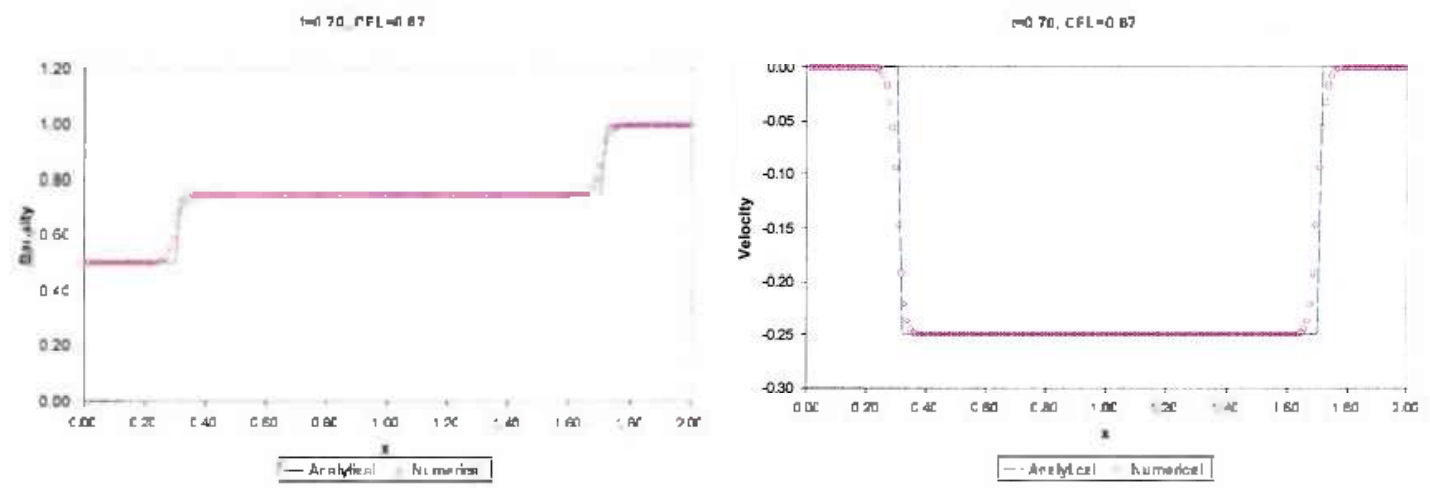

Figure 7. Comparison for the Linearized Gas Dynamics equations (resc 2).
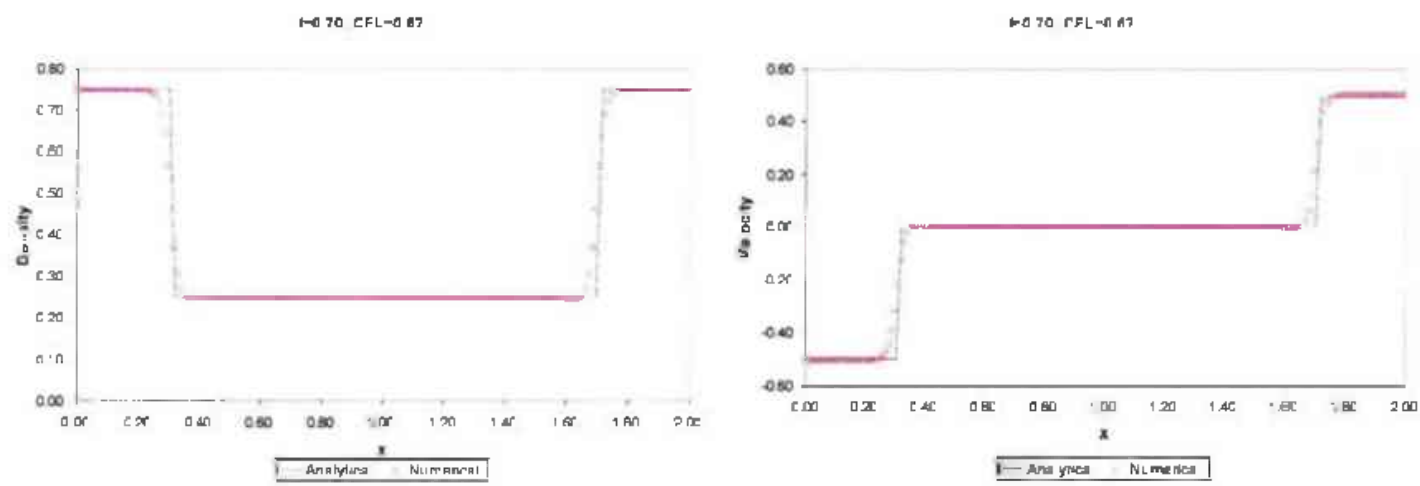

Figure 8. Comparison for the Linearized Gas Dynamics equations (test 3).

\subsection{Homogeneous Non-linear System - Euler Equations}

The non-linear hyperbolic laws that govern the motion of an inviscid non-heat conducting gas are the timedependent Euler equations (44). The Euler equations are of interest because many of the major elements of fluid dynarnics are incorporated in them $\mid 7]$, such as shock waves or contacl surfaces. 


$$
\begin{aligned}
& \frac{\partial \rho}{\partial t}+\frac{\partial \rho u}{\partial x}=0 \\
& \frac{\partial \rho u}{\partial t}+\frac{\partial\left(\rho u^{2}+p\right)}{\partial x}=0 \\
& \frac{\partial E}{\partial t}+\frac{\partial[u(E+p)]}{\partial x}=0
\end{aligned}
$$

The so called shock tube problem is a closed tube initially divided into a high-pressure section and a low-pressure secrion by a fixed diaphragm: making both sections to be initially at velocity zero [13]. Then the diaphragm is removed, allowing the discontinuity to propagate. This problem was solved numerically for two different initial conditions (Table 5), in the spatial domain $|0,1|$, using $400 \mathrm{cel} \mid \mathrm{s}$, and a CFL number 0.5 .

Table 5. Specificarions of the different tests. Euler equations.

\begin{tabular}{|c|c|c|c|c|c|c|c|c|}
\hline Test & $\mathbf{x}_{0}$ & Time & $\mathbf{U}_{\mathbf{1} \mathbf{L}}$ & $\mathbf{U}_{\mathbf{2} \mathbf{L}}$ & $\mathbf{U}_{\text {3 L }}$ & $\mathbf{U}_{\mathbf{1} \mathbf{R}}$ & $\mathbf{U}_{\mathbf{2} \mathbf{R}}$ & $\mathbf{U}_{\mathbf{3} \mathbf{R}}$ \\
\hline $\mathbf{1}$ & 0.5 & 0.25 & 1.000 & 0.000 & 2.500 & 0.125 & 0.00 & 0.250 \\
\hline $\mathbf{2}$ & 0.6 & 0.10 & 0.445 & 0.298 & 8.919 & 0.500 & 0.00 & 1.427 \\
\hline
\end{tabular}

The numerical results are presenred in Figures 9 and 10 , where they are compared against the exact solution obtained from an exact solver described by Toro [13].
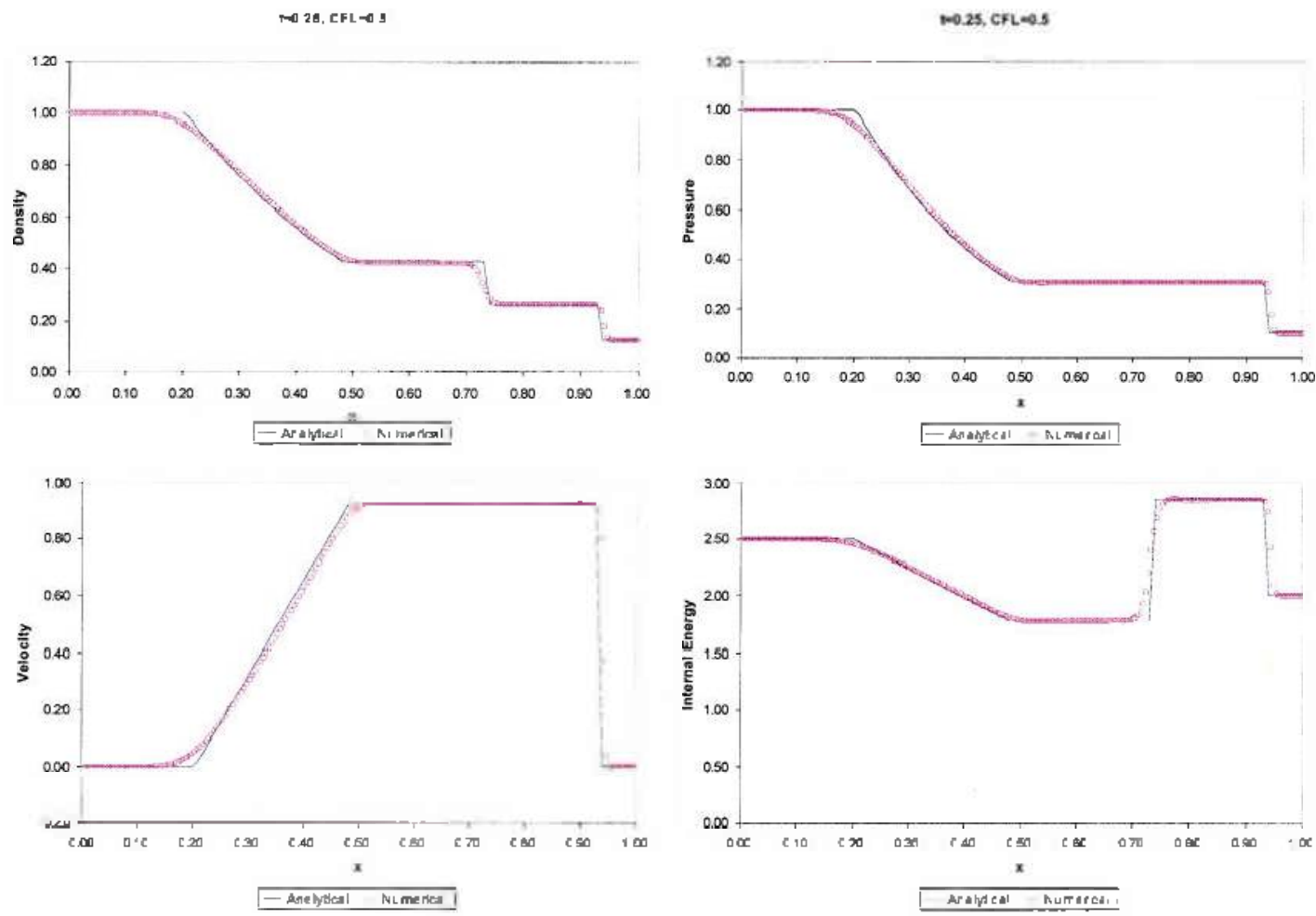

Figure 9. Comparison for the Euler equations (test 1). 

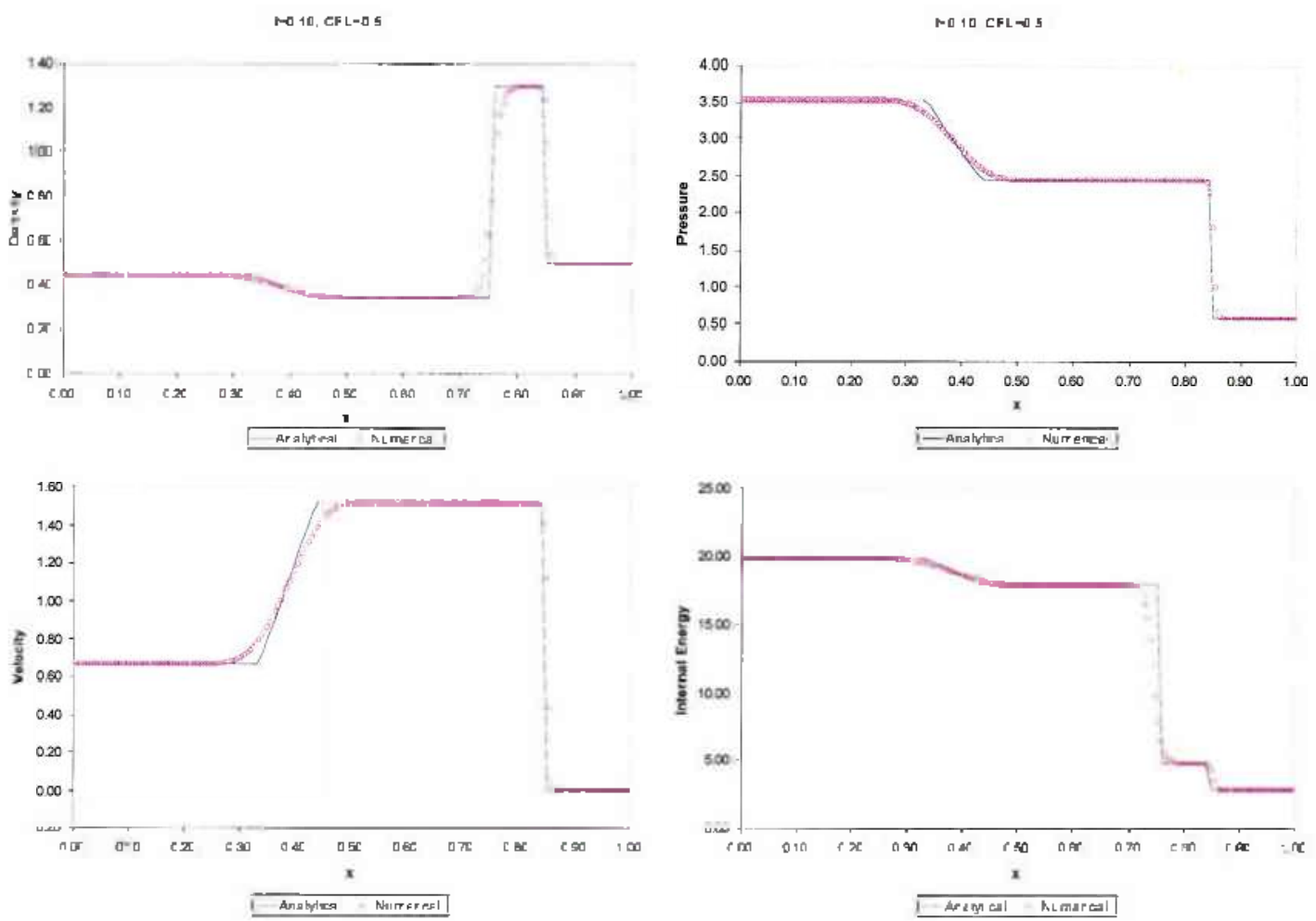

Figure 10. Comparison for the Euler equations (lest 2).

3.5. Non-homogeneous Scalar Case-Model Splitting Equation

At this point the goal is to solve non-homogeneous conservation laws. Let us consider the simplest model hyperbolic equation (26) with the ICs (45).

$$
\text { IC }: u(x, 0)=u_{n}(x)=\left|\begin{array}{ll}
1.0 & \text { if } x \leq 0.5 \\
0.0 & \text { if } x>0.5
\end{array}\right|
$$

The IVP (26)-(45) was solved numerically in the spatial domain $[0,2.5]$ using 250 cells, and a CFL number 0.99 , while the constant values were set to be: $a=1$ and $\eta-1$. The solution was computed for three different output times $(0.5,1.0$ and 1.5$)$ using both the first- and second-order accurate splitting schemes. The numerical solutions are compared against the exact profiles in Figure 11. 

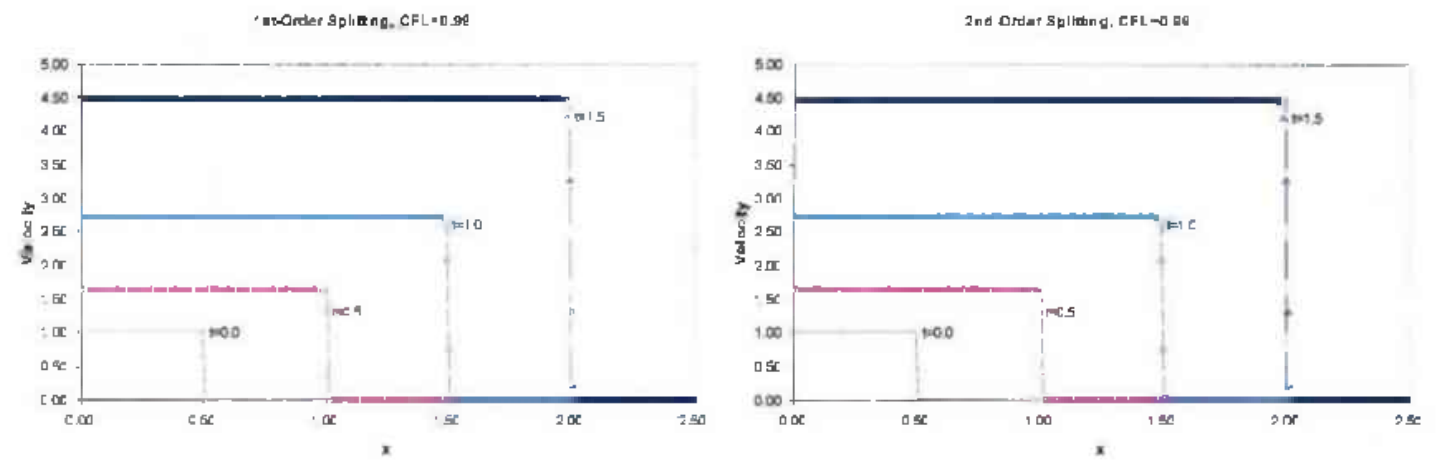

Figure 1 1. Comparison for the Model equation ( $\mathrm{I}^{\mathrm{s}}$ - and $2^{\text {od }}$-order splitting).

\subsection{Non-homogeneous System-Two-Coupled Model Spltting Equations}

In order to validate the solver, as we have done in the previous problems, we need to compare the numerical results with their corresponding exact solutions. Therefore. due to the lack of problems with analytical solution, we created our own system of equations, for which we know the exact solution. The derivation is as follows:

I.et us write two independent model splitting equations of the form (26), in two different variables $u$ and $v$, these are:

$$
\left.\begin{array}{l}
\frac{\partial u^{*}}{\partial t}+a_{1} \frac{\partial u^{*}}{\partial x}=\eta_{1} u^{*} \\
I C: \quad u^{*}(x, 0)=u_{\circ}^{*}(x)
\end{array}\right\}
$$

The corresponding exact solutions of (46) and (47) are given by:

$$
\begin{aligned}
& u^{*}(x, t)=u_{0}^{*}\left(x-a_{1} t\right) e^{\eta_{1} t} \\
& v^{*}(x, t)=v^{*}\left(x-a_{2} t\right) e^{\eta_{t} t}
\end{aligned}
$$

Now we define the new coupled variables $U_{1}$ and $U_{2}$ as:

$$
\begin{aligned}
& U_{1}=u^{*}+v^{*} \\
& U_{2}=u^{*}-v^{*}
\end{aligned}
$$

Hence, we can express $u$ and $v$ as a function of the new variables $U_{1}$ and $U_{2}$ like:

$$
\begin{aligned}
& u^{\prime}=\frac{U_{1}+U_{2}}{2} \\
& v^{\prime}=\frac{U_{1}-U_{2}}{2}
\end{aligned}
$$


Now, to generate the new coupled PDEs in terms of $U_{1}$ and $U_{2}$ we add equations (46) and (47), which after some simplifications becomes (54).

$$
\begin{gathered}
\frac{\partial U_{1}}{\partial t}+\frac{\partial\left(\frac{a_{1}}{2}\left(U_{1}+U_{2}\right)+\frac{a_{2}}{2}\left(U_{1}-U_{2}\right)\right)}{\partial x}=\frac{\eta_{1}\left(U_{1}+U_{2}\right)}{2}+\frac{\eta_{2}\left(U_{1}-U_{2}\right)}{2} \\
\frac{\partial U_{1}}{\partial t}+\frac{\partial \mid\left(\frac{a+a_{2}}{2}\right) U_{1}+\left(\frac{a-a_{2}}{2}\right) U_{2} J}{\partial x}=\left(\frac{n+\eta_{1}}{2}\right) U_{1}+\left(\frac{n-\eta_{2}}{2}\right) U_{2}
\end{gathered}
$$

Similarly, subtracting (47) from (46) one obtains (55) as:

$$
\begin{gathered}
\frac{\partial U_{2}}{\partial t}+\frac{\partial\left(\frac{a}{2}\left(U_{1}+U_{2}\right)-\frac{a_{1}}{2}\left(U_{1}-U_{2}\right)\right)}{\partial x}=\frac{\eta_{1}\left(U_{1}+U_{2}\right)}{2}-\frac{\eta_{2}\left(U_{1}-U_{2}\right)}{2} \\
\frac{\partial U_{2}}{\partial t}+\frac{\partial\left[\left(\frac{a-a_{2}}{2}\right) U_{1}+\left(\frac{a+a_{2}}{2}\right) U_{2}\right\rfloor^{\prime}}{\partial x}=\left(\frac{\left(1-\eta_{2}\right.}{2}\right) U_{1}+\left(\frac{\eta_{1}+\eta_{2}}{2}\right) U_{2}
\end{gathered}
$$

Finally, the coupled system of equations (54) and (55) can be written in conservative form as:

$$
\frac{\partial U}{\partial x}+\frac{\partial F(U)}{\partial x}=\stackrel{r}{Q}(U)
$$

where:

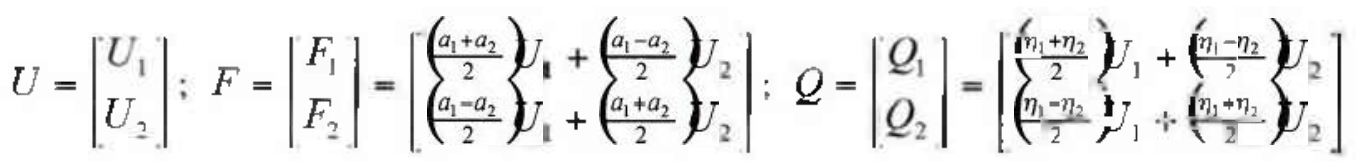

From the transformations (50) and (51) it is clear that the ICs of the system are:

$$
\begin{aligned}
& U_{1}(x, 0)=U_{1 o}(x)-u_{o}^{*}(x)+v_{o}^{*}(x) \\
& U_{2}(x, 0)=U_{2 o}(x)=u_{o}^{*}(x)-v_{o}^{*}(x)
\end{aligned} \mid
$$

And the exact solution of the system (57) can be written as:

$$
\begin{aligned}
& U_{1}(x, t)=u^{*}(x, t)+v^{*}(x, t)=u_{0}^{*}\left(x-a_{1} t\right) e^{\eta_{1} t}+v_{v}^{*}\left(x-a_{2} t\right) e^{\eta_{1} t} \\
& U_{2}(x, t)=u^{*}(x, t)-v^{*}(x, t)=u_{0}^{*}\left(x-a_{1} t\right) e^{\eta^{n t}}-v_{0}^{*}\left(x-a_{2} t\right) e^{\eta_{2}^{\prime}}
\end{aligned}
$$

Eigenstructure of the System. Even though the solver does not need it, to completely define our own problem, here we included the eigenstructure of the system, which could be useful for future studies that may use this system as a benchmark problem. The Jacobian matrix is given by:

$$
A(U)=\frac{\partial F}{\partial U}=\left[\begin{array}{c}
\left(\frac{a_{1}+a_{2}}{2}\right) \\
\left(\frac{a_{1}-a_{2}}{2}\right)
\end{array}\left(\begin{array}{c}
\frac{a_{1}-a_{2}}{2} \\
\left(\frac{a_{1}+a_{2}}{2}\right)
\end{array}\right]\right.
$$


The eigenvalues of the matrix $A(U)$ are obtained from the characteristic equation, which is satisfied when the following determinant vanishes:

$$
|A-\lambda I|=\left|\begin{array}{cc}
\left(\frac{a_{1}+a_{3}}{2}\right)-\lambda & \left(\frac{a_{-}-a_{1}}{2}\right) \\
\left(\frac{a_{1}-a_{2}}{2}\right) & \left(\frac{a_{1}+a_{2}}{2}\right)-\lambda
\end{array}\right|=0
$$

Expression (60) can be written as the second-order polynomial (61)

$$
\begin{gathered}
{\left[\left(\frac{a_{1}-a_{i}}{2}\right)-\lambda\right]-\left(\frac{a_{1}-a_{2}}{2}\right)=0} \\
\left(\frac{a+a_{1}}{2}\right)-2\left(\frac{a+a_{3}}{2}\right) \lambda+\lambda^{2}-\left(\frac{a_{1}-a_{1}}{2}\right)=0 \\
\lambda^{2}-2\left(\frac{a_{1}+a_{3}}{2}\right) \lambda+\left(\frac{a_{1}+a_{1}}{2}\right)-\left(\frac{a_{1}-a_{2}}{2}\right)=0
\end{gathered}
$$

This is solved using the quadratic equation, to produce the eigenvalues (62).

$$
\begin{gathered}
\lambda=\frac{\left.2\left(\frac{a-a_{3}}{2}\right) \pm \sqrt{\left[2\left(\frac{a_{1}+a_{1}}{2}\right)\right]-4\left[\left(\frac{a_{1}+a_{3}}{2}\right)-\left(\frac{a_{1}-a_{3}}{2}\right)\right.}\right]}{2} \\
\lambda=\frac{2\left(\frac{a+a_{1}}{2}\right) \pm \sqrt{4\left(\frac{a-a_{1}}{2}\right)}}{2} \\
\lambda=\frac{2\left(\frac{a+a_{1}}{2}\right) \pm 2\left(\frac{a-a_{2}}{2}\right)}{2} \\
\lambda_{1}=a_{1} ; \quad \lambda_{3}=a_{2}
\end{gathered}
$$

Now, by definition, the right eigenvectors are obtained as (9), which in matrix form is:

$$
\left|\begin{array}{cc}
\left(\frac{a-a_{2}}{2}\right) & \left(\frac{a-a_{1}}{2}\right) \\
\left(\frac{a-a_{1}}{2}\right) & \left(\frac{a+a}{2}\right)
\end{array}\right|\left|\begin{array}{l}
R_{1}^{(n)} \\
R_{2}^{(n)}
\end{array}\right|=\lambda\left|\begin{array}{l}
R_{1}^{(j)} \\
R_{2}^{(o)}
\end{array}\right|
$$

Producing the two linearly dependent equations:

$$
\begin{aligned}
& \left(\frac{a_{1}+a_{3}}{2}\right) \boldsymbol{R}_{1}^{(i)}+\left(\frac{a_{-}-a_{2}}{2}\right) R_{2}^{(i)}=\lambda_{1} R_{1}^{(i)} \\
& \left(\frac{a_{1}-a_{3}}{2}\right) R_{1}^{(i)}+\left(\frac{a_{1}+a_{2}}{2}\right) R_{2}^{(t)}=\lambda_{1} R_{2}^{())}
\end{aligned}
$$

Since, what we have are two linearly dependent equations, the corresponding right eigenvector of $\lambda_{1}=a_{1}$ is obtained by setting the norm of the vector to be $R_{1}^{\prime \prime \prime}=1$, which leads the second component of the eigenvector to be:

$$
R_{:}^{(1)}=\frac{-\left(\frac{-a+a}{2}\right)}{\left(\frac{a_{1}-a_{2}}{3}\right)}=\frac{a_{1}-a_{2}}{a_{1}-a_{2}}=1
$$

The same procedure is applied to obtain the corresponding right eigenvector of $\lambda_{2}=a_{2}$, yielding to: 


$$
R^{(1)}=\left|\begin{array}{l}
1 \\
1
\end{array}\right| ; \quad R^{(2)}=\left|\begin{array}{c}
1 \\
-1
\end{array}\right|
$$

Numerical Test /. Having the equations (46) and (47), with initial conditions:

$$
\begin{aligned}
u^{\prime}(x, 0)=u_{s}^{\prime \prime}(x) & = \begin{cases}2 & \text { if } x \leq 0.5 \\
0 & \text { if } x>0.5\end{cases} \\
v^{\prime}(x, 0)=v_{\Delta}^{*}(x) & =\left\{\begin{array}{ll}
1 & \text { if } x \leq 0.5 \\
0 & \text { if } x>0.5
\end{array}\right\}
\end{aligned}
$$

From (57) we obtain the ICs of the system (56) as:

$$
\begin{array}{r}
U_{1}(x, 0)=U_{10}(x)=\mid \begin{array}{ll}
3 & \text { if } x \leq 0.5 \\
0 & \text { if } x>0.5
\end{array} \\
U_{2}(x, 0)=U_{20}(x)=\left\{\begin{array}{ll}
1 & \text { if } x \leq 0.5 \\
0 & \text { if } x>0.5
\end{array} \mid\right.
\end{array}
$$

Then, the problem (56), with ICs (66), was solved in the spatial domain $[0,5.0]$ using 500 cells, and a CFL number 0.99. The constants used are: $a_{1}=1.0, a_{2}=2.0, \eta_{1}=2.0, \eta_{2}=1.0$. The numerical solutions are computed for three output fimes $(0.5,1.0$ and 1.5$)$ using both the first- and second-order accurate splilting schemes, and they are compared against the exact profiles in Figures 12 and 13.
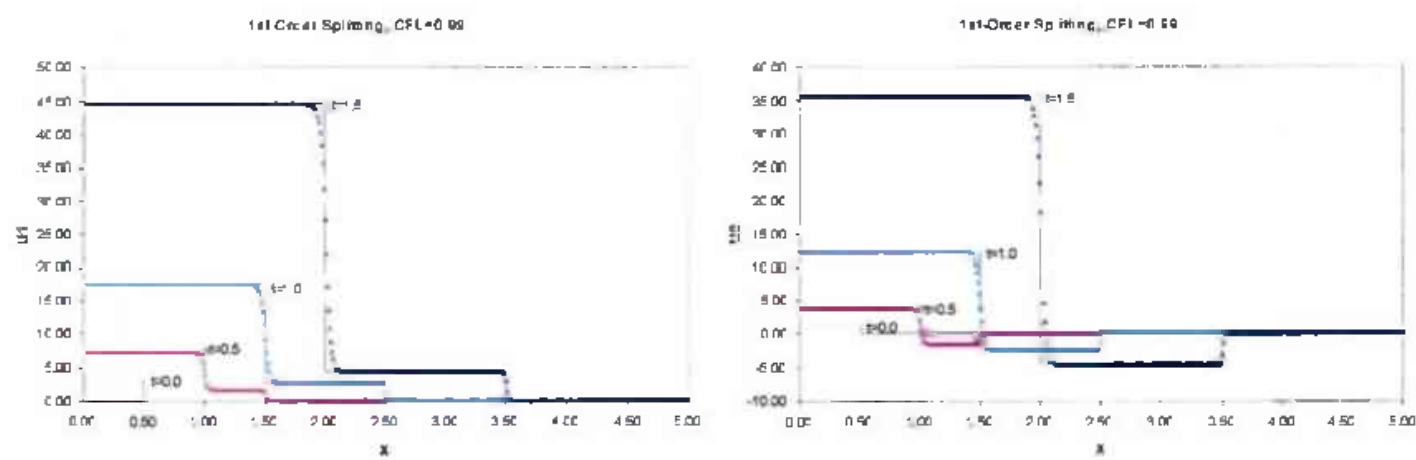

Figure 12. Comparison for the lwo-coupled Model cquations using $1^{\text {st }}$-order splitting (test 1 ). 


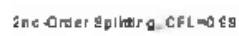

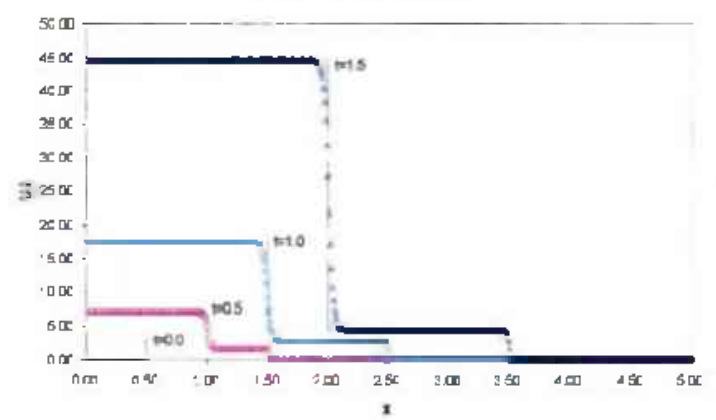

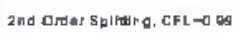

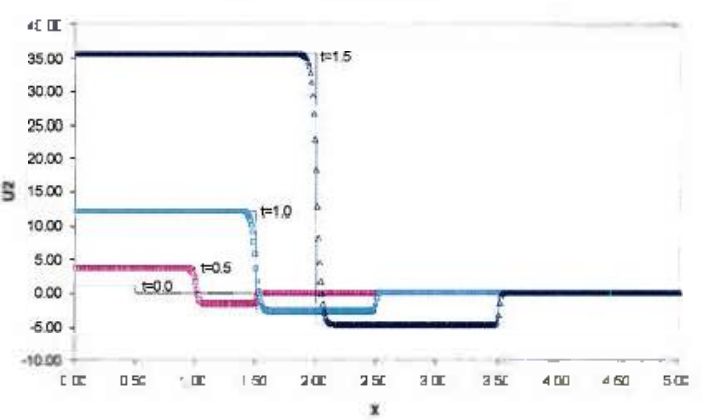

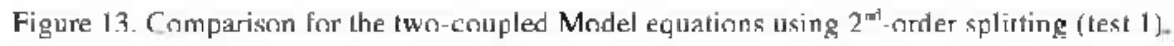

Numerical Test 2: This problem is exactly similar to the previous one. hut the constants used are: $a_{1}=a_{2}=1.0 . \eta_{1}=20 . \eta_{2}=1.0$. Note that by making $a_{1}=a_{2}$, the system of equations is only coupled trough the source terms. This is:

$$
\frac{\partial U}{\partial x}+\frac{\partial F(U)}{\partial x}=Q(U)
$$

where:

$$
U=\left[\begin{array}{l}
U_{1} \\
U_{2}
\end{array}\right] ; \quad F=\left[\begin{array}{l}
F_{1} \\
F_{2}
\end{array}\right]=\left[\begin{array}{l}
a_{1} U_{1} \\
a_{1} U_{2}
\end{array}\right] ; \quad Q=\left[\begin{array}{l}
Q_{1} \\
Q_{2}
\end{array}\right]=\left[\begin{array}{l}
\left(\frac{\eta_{1}+\eta_{2}}{2}\right) U_{1}+\left(\frac{\eta_{1}-\eta_{2}}{2}\right) U_{2} \\
\left.\frac{\eta_{1}-\eta_{2}}{2}\right)_{1}+\left(\frac{\eta_{1}+\eta_{2}}{2}\right) U_{2}
\end{array}\right]
$$

The solution was computed for three different output times $(0.5,1.0$ and 1.5$)$ using both the first- and second-order accurate splitting schemes. The numerical solutions are compared against the exact ones in Figures 14 and 15.
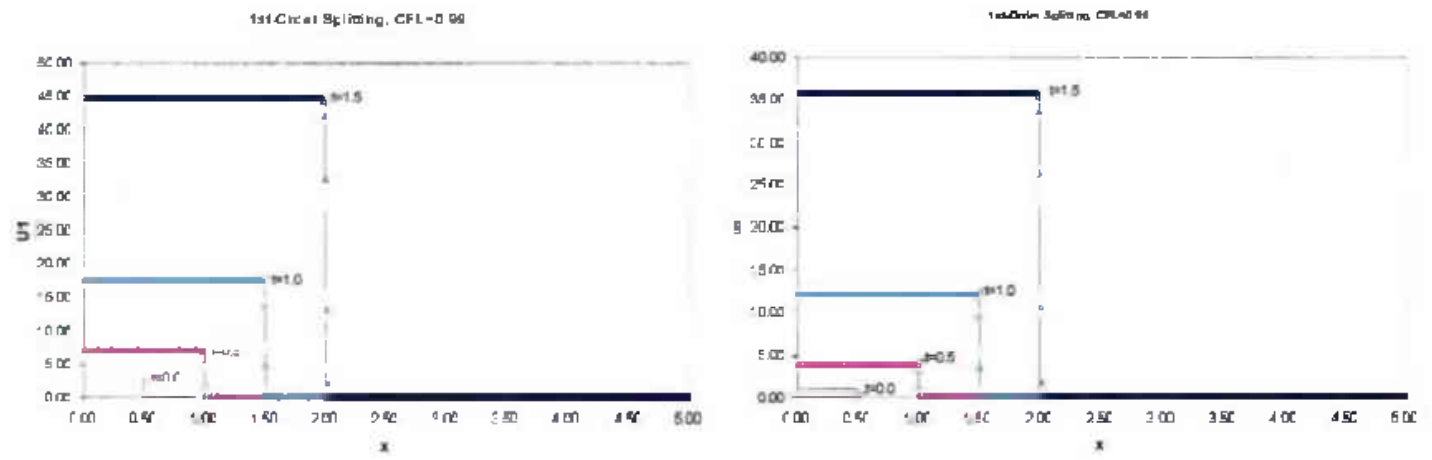

Figure 14. Comparison for the Two-coupled Model equations using $1^{\text {st }}$-order splitting (test 2). 

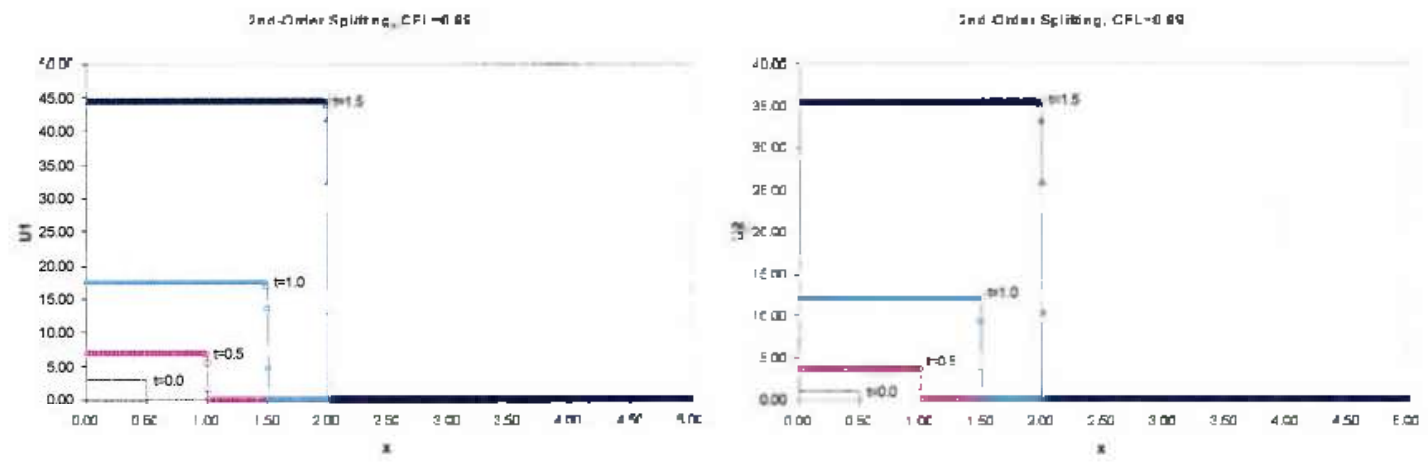

Figure 1.15. Comparison for the Two-coupled Model equations using $2^{\text {ad }}$-order sphitting (test 2).

\section{CONCLUSIONS}

An efficient, maintainable, transportable and useful Generalized PDEs Solver, that minimizes data requirements, has been developed. The splitting approach is used to handle the presence of forcing functions with the Harten's TVD scheme used for solving the homogeneous system of PDEs, and the fifth-order Cash \& Karp Embedded RungcKutta method for the source problem.

The program was validated by solving the most general case of a syslem of hyperbolic PDEs and its simplifications, using classical Benchmark problems to compare the numerical results against their analytical counterparts. Moreover, the ability of the solver to handle the cases of conservative variables with negative signs, and mixed signs was validated by performing different tests for the Linearized Gas Dynamics equations.

All our experiments agree with Harten in the sense that the arîthmetic average works as well as the Roe's linearization when calculating the average Jacobian matrix, needed it to obtain the eigenestructure and other functions required by the Harten's modified flux at the intercell, although Roe qualified that choice as unsatisfactory,

The Detonation Analogue proposed by Fickett was found to be inappropriate to validate our solver. Hence, a new non-homogeneous system with its analytical solution is presented. This system of two-coupled model equations was used as a Benchmark problem for this solver, and can be useful for future studies.

The results generated by the splitting scheme for the non-homogeneous cases match the corresponding exact solutions quite well. Moreover, for the experiments performed, no appreciable difference can be ohserved between the results given by the first- and second-order splitting approaches, neither in the scalar case, nor in the system of equations. This fact motivates us to recommend the use of the first-order splitting because it requires one less evaluation of the ODE solver for each grid point at each time step, reducing the computation time. 


\section{APPENDIX A. NOMECLATURE}

$a:$ speed of sound

$a_{,}^{F}$ : constant coefficient given by Cash and Karp. Table 3

$A(U)$ : Jacobian matrix

$\widetilde{A}(U)$ : average Jacobian matrix

$C:$ constant

$C_{c f}:$ CFL coefficient

$c_{p}$ : specific heat at constant pressure

$c$. : specific heat at constant volume

$d_{y}{ }^{p}$ : constant coefficient given by Cash and Karp. Table 3

$e:$ specific internal energy

$E$ : total energy per unit volume

$f_{r+f}^{l f}$ : Harten's modified numerical flux defined by Equation (15)

$F(U)$ : vector of physical fluxes

$F_{1}$ : physical flux of $\mathrm{j}^{\text {th }}$ equation

$F_{i, s_{1}}^{n}:$ numerical flux at the intercell $(i, i+1)$ at time level $n$

$F_{i}^{f}$ : functions in the Runge-Kutta method. Equation (38)

$g^{k}$ : function $g$ corresponding to the $\mathbf{k}^{\text {th }}$ eigenvalues at the $\mathrm{j}^{\text {th }}$ grid point. Equation (18)

$\tilde{g}_{j+\frac{1}{2}}^{2}$ : function $\tilde{g}$ corresponding to the $\mathbf{k}^{\text {Lb }}$ elgenvalues at the intercell $(j, j+1)$. Equation $(18)$

$H$ : total specific enthalpy

$I$ : identicy matrix

$L$ : spatial domain

$N$ : number of grid points

$O^{(\Delta)}$ : ODF operator applied on a time step $\Delta t$

$p$ : pressure

$P^{|\star|}$ : PDE operator applied on a time step $\Delta t$

$Q(U)$ : vector of forcing functions

$R^{(k)}: \mathrm{k}^{\text {th }}$ eigenvector of the system

$R_{j}^{(k)}$ : component $\mathrm{j}^{\text {th }}$ of the $\mathrm{k}^{\text {th }}$ eigenvector

RHS : right hand side of the conservative equation

$s_{j+\frac{\xi}{3}}^{k}$ sign of function $\widetilde{g}_{,+\frac{1}{3}}^{k}$. Equation (18)

$S_{\text {max }}^{n}$ : largest wave speed in the domain at time tevel $n$

$t$ : time

$u$ : velocity

$u^{*}:$ function in $x$ and $t$

$U$ : vector of conservative variables

$U$, : conservative variable of $\mathrm{j}^{\text {th }}$ equation 
$U_{1}$ : constant value of the conservative variable in the left side

$U_{R}$ : constant value of the conservative variable in the right side

$U_{i}^{n}$ : conservative variable in the $\mathrm{i}^{\text {th }}$ grid point at time level $n$

$v^{-}$: function in $x$ and $t$

$\bar{v}_{j+t}$ : function $\bar{v}$ at the intercell $(j, j+1)$. Equation (18)

$x$ : æxial-coordinate

Greek Letters

$v_{j+j}^{i}$ : function $v$ at the intercell $(j, j+1)$. Equation (18)

$\alpha_{j+t}^{k}$ : wave strength of the $\mathrm{k}^{\text {th }}$ eigenvalues at the intercell $(j, j+1)$. Equation (18)

$\beta$ : function defined by Equation (12)

$\varepsilon$, epsilon corresponding to the $j^{\text {it }}$ conservative variable

$\gamma$ : ratio of specific heats

$\lambda$ : eigenvalue

$\lambda_{j}: j^{\text {th }}$ eigenvalue of the system

$\mu(v)$ : function in the viscosity cocfficient defined by Equation (19)

$\mu^{1}$ : function in the viscosity coefficient defined corresponding to the $\mathrm{k}^{\text {th }}$ eigenvalues

$\rho:$ density

$\eta$ : conscan1 parameter in Equation (26).

$\sigma: \Delta / \Delta x$

$\delta:$ constant in the function $\mu(v)$, defined by Harten. Equation (19)

$\Delta t:$ uniform time step

$\Delta x$ : uniform grid cell size

Subscripts

$j+\frac{1}{2}$ : intercell between the $j$ and $j+I$ grid points

$o:$ initial conditions

Superscripts

$k$ : referring to the $\mathrm{k}^{\text {th }}$ eigenvalue

$m$ : number of equations

$n: n^{\text {th }}$ time level 


\section{REFERENCES}

1. Toro, HiF.: Riemann Solvers and Numerical Methods for Fluid Dynamics, A Practical Iniroducrion, Springer, Manchester - U.K. , Second Edicion, 1999

2. Courant, R., Friedrichs, K. O. and I.wy, H.: Uber die Partiellen Differenzengleichungen der Mathematischen Physik, Marh. Ann, Volume 100. 32-74. (Translated to: On the Partial Difference Equarions of Mathemaical Physics, IBM J. Res. Dev., Volume I1, 215-234, 1967.

3. Heath, M.: Scienific Computing, An Introductory Survey, McGraw-Hill, PA. Second Edition. 2002.

4. Harten. A.: High Resolution Schemes for Hyperbolic Conservation Laws, Journal of Compurational Phyrics, Volurne 49. 357-393, 1983.

5. Hirsch, Ch.: Numericat computation of internal and exrernal flows, Volume 2, John Wiley and Sons, New York, NY, 1988.

6. Roc, P. L.: Approximate Riemann Solvers, Parameter Vectors, and Difference Schernes, Journal of Computational physics, Volume 43. 357-372, 19B1

7. Tannehill, J. C. Anderson, D. A. and Pletcher, R. H.: Compuational Fivid Mechanics and Heat Transfer, Taylor \& Francys, Philadelphia, PA, Second Edirion. 1997

8. Fehlberg, E.: Classical Fifth. Sixth., Seventh., and Eighth Order Runge-Kurto Formulas with Stepsize Control, National Aeronaurics and Space Administration (NASA), Washing1on. DC. 1968.

9. Press. W. H.. Teukalsky, S. A., Venerling, W. T. and Flannery, B, P.: Numerical Recipes in Fortran. The Art of Scientific Computing. Second Edizion, Cambridge University Press, New York. NY, 1994

10. Casb, J. R. and Karp, A. H.: A Voriabte Order Runge-Kulta Method for Initial Value Problems wihh Rapidly Varying Right-Hand Sides, ACM Trans. Math. Sofiw., Volume 16. 201-222. 1990

11. Fickert, W.: Introduczion ro Detonation Theory. University of Califormia Press, Berkley-1.os Angeles-1 nndon, 1985.

12. Clarke. I. F., Kami. S., Quirk. J. J., Roe, P. L., Simmonds. L. G. and Toro, E. F.: Numerical Computation of Two Dimensional Unsieady Delonation Waves in High Energy Solids. Journal of Computotional Physics, Volume 106, 215-233, I993.

13. Anderson, I.D. Computationel Fiuid Dynamics. The Bosics with Applirations, McGraw-Hill, Inc.. Singapore. I995 